\title{
Stereoselective Synthesis of Highly Substituted Tetrahydropyrans through an Evans Aldol-Prins Strategy
}

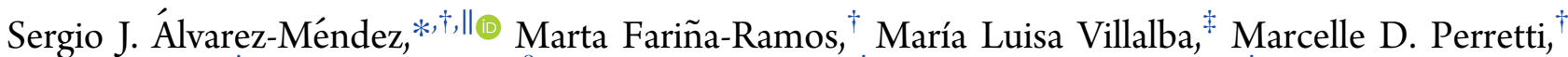 \\ Celina García, ${ }^{\dagger}$ Laila M. Moujir, ${ }^{\S}$ Miguel A. Ramírez, ${ }^{\dagger}$ and Víctor S. Martín* ${ }^{*}{ }^{\dagger}$
}

†Instituto Universitario de Bio-Orgánica “Antonio González” (IUBO-AG), Centro de Investigaciones Biomédicas de Canarias (CIBICAN), Departamento de Química Orgánica, Universidad de La Laguna (ULL), Avda. Astrofísico Francisco Sánchez 2, 38206 San Cristóbal de La Laguna, Tenerife, Spain

${ }^{\ddagger}$ Laboratorio de Investigación y Desarrollo de Bioactivos (LIDeB), Departamento de Ciencias Biológicas, Facultad de Ciencias Exactas, Universidad Nacional de La Plata, 47 \& 115, B1900AJI La Plata, Buenos Aires, Argentina

${ }^{\S}$ Departamento de Bioquímica, Microbiología, Biología Celular y Genética, Facultad de Farmacia, Universidad de La Laguna (ULL), Avda. Astrofísico Francisco Sánchez s/n, 38206 San Cristóbal de La Laguna, Tenerife, Spain

\section{Supporting Information}

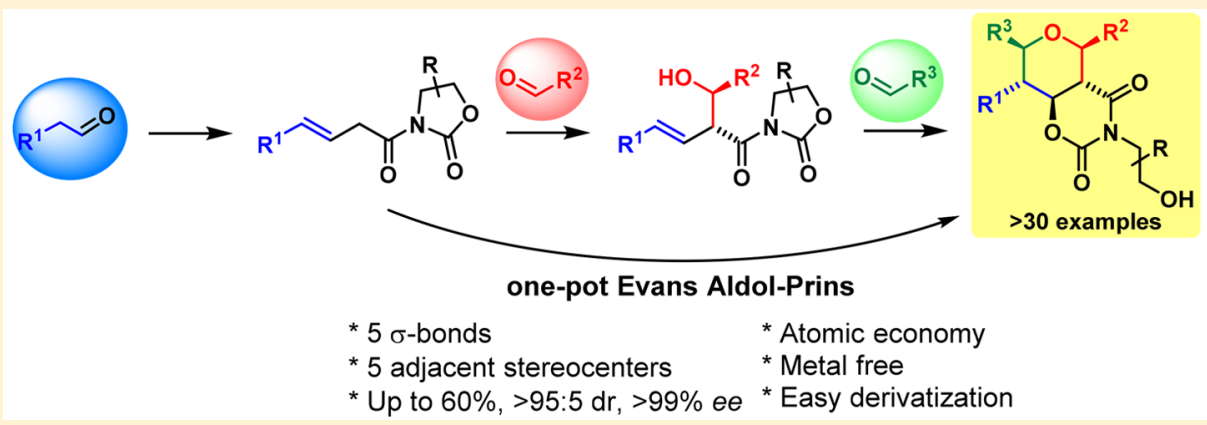

ABSTRACT: A direct and general method for the synthesis of naturally occurring 2,3,4,5,6-pentasubstituted tetrahydropyrans has been developed, employing $\beta, \gamma$-unsaturated $N$-acyl oxazolidin-2-ones as key starting materials. The combination of the Evans aldol addition and the Prins cyclization allowed the diastereoselective and efficient generation of the desired oxacycles in two fashions: a one-pot Evans aldol-Prins protocol, in which five new $\sigma$ bonds and five contiguous stereocenters were straightforwardly generated, and a two-step version, which additionally permitted the isolation of $\beta, \gamma$-unsaturated alcohol precursors bearing an $\mathrm{N}$-acyl oxazolidin-2-one in the $\alpha$ position. From these alcohols were also obtained halogenated pentasubstituted tetrahydropyrans as well as 2,3,4,5-tetrasubstituted tetrahydrofurans, shedding light on the mechanism of the process. Computational studies were consistent with the experimental findings, and this innovative Evans aldol-Prins strategy was performed for the preparation of a battery of more than 30 densely substituted tetrahydropyrans, unprecedentedly fused to a 1,3-oxazinane-2,4-dione ring, both in a racemic fashion and in an enantiomeric fashion. These novel molecules were successfully submitted to several transformations to permit simple access to a variety of differently functionalized tetrahydropyrans. Most of these unique molecules were evaluated for their antimicrobial activity against Gram-positive and Gram-negative bacteria and the yeast Candida albicans, and some structure-activity relationships were established.

\section{INTRODUCTION}

The tetrahydropyran (THP) motif is commonly found in biologically active secondary metabolites isolated from marine and terrestrial sources, such as those shown in Figure 1, and is found to be part of complex cyclic polyether systems. ${ }^{1}$ For example, 2,3,5-trisubstituted THPs can be found in morinols A and B, isolated in 1999 from Morina chinensis, a plant employed in traditional Chinese medicine. ${ }^{2}$ Morinols and some derivatives show antiproliferative, ${ }^{3}$ antimicrobial, ${ }^{4}$ and antifungal activity. ${ }^{5}$ More substituted THPs can be found in clavosolides A and B, which exhibit two 2,3,4,6-tetrasubstituted THPs in their structures. They were isolated from the cytotoxic extract of the sponge Myriastra clavosa from the
Philippines. ${ }^{6}$ The same substitution and stereochemical pattern appears in the tetrasubstituted THP borne by members of the polycavernoside family of toxins, isolated from the red alga Gracilaria edulis (also known as Polycavernosa tsudai).? Kendomycin, which was isolated from several Streptomyces strains, shows a 2,3,4,5,6-pentasubstituted THP with the substituent at $\mathrm{C}_{5}$ adopting an axial disposition. This compound acts as an endothelin receptor antagonist and also exhibits cytotoxicity against multiple human cell line and antiosteoporotic and antibiotic activities. ${ }^{8}$ Phorboxazoles A and B were

Received: May 9, 2018

Published: July 23, 2018 


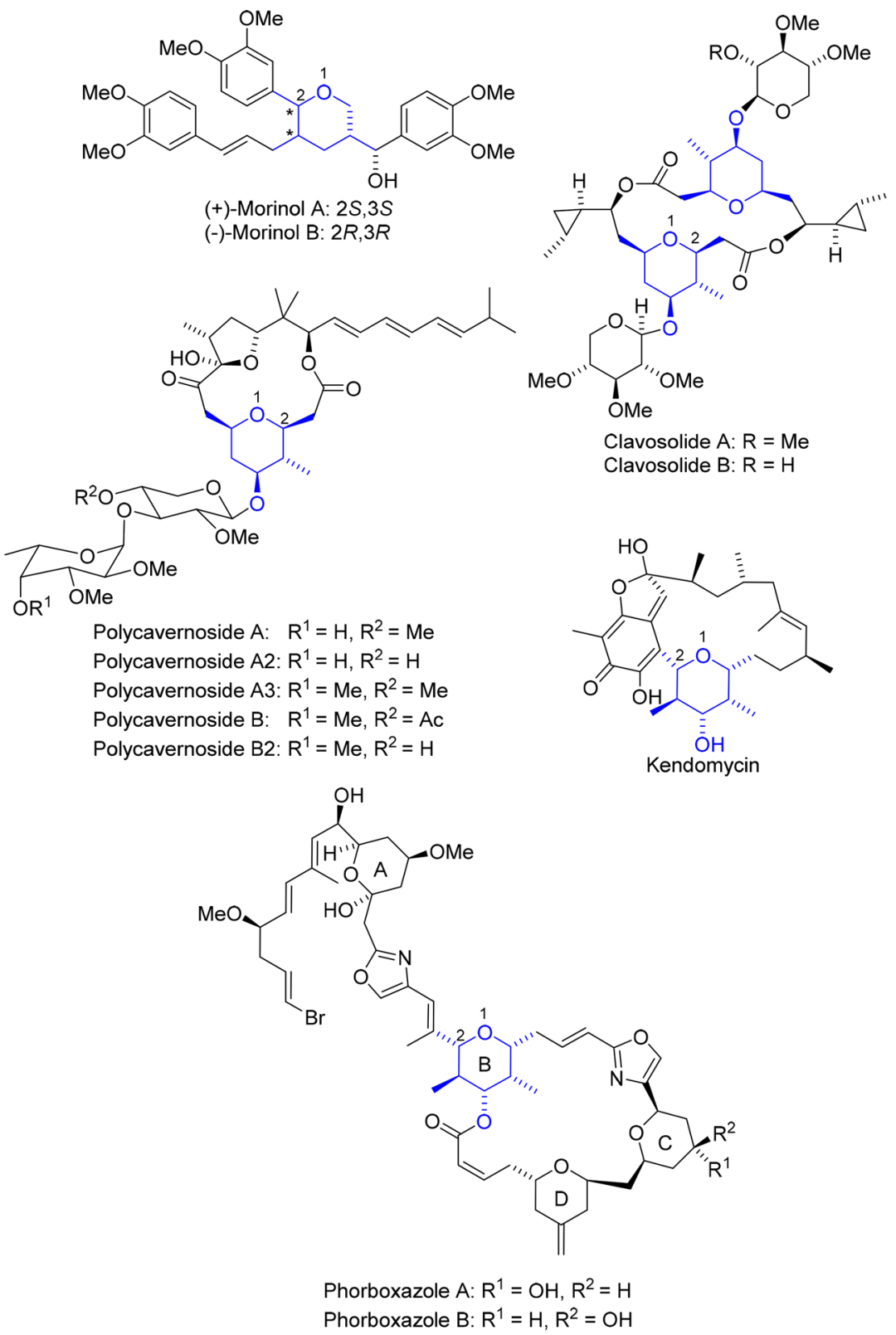

Figure 1. Examples of natural products containing nonfused densely substituted THPs.

isolated from Indian Ocean sponge Phorbas sp. and show antitumor activity and antifungal activity against Candida albicans. ${ }^{9}$ Four THP rings appear in their structures, underlining the presence of the THP labeled as B (Figure 1) with substituents at all its positions and the same substitution pattern found in kendomycin. Besides being part of bioactive natural products, it has been demonstrated that the THP ring can even improve the efficacy of antiviral drugs ${ }^{10}$ and can show bioactivity itself, such as antinociceptive activity, ${ }^{11}$ serotonin and norepinephrine transporter inhibitory activity, ${ }^{12}$ antimicrobial activity by the inhibition of bacterial topoisomerase, ${ }^{13}$ and antiproliferative activity. ${ }^{14}$

The number of natural products containing a THP ring has encouraged the development and application of many synthetic strategies, such as Pd-catalyzed oxaheterocyclization, ${ }^{15}$ the Petasis-Ferrier union/rearrangement tactic, ${ }^{16}$ Michael-like reactions, ${ }^{17} \mathrm{~S}_{\mathrm{N}}$-mediated and metal-promoted cyclizations, ${ }^{17}$ ester enolate Claisen rearrangement, ${ }^{18}$ ring expansion of tetrahydrofurans, ${ }^{18}$ 1,5-cyclization, ${ }^{18}$ iodolactonization, ${ }^{18}$ epoxide opening-ring closure reactions, ${ }^{18}$ etc. ${ }^{19}$ Among the existing tactics, the Prins cyclization ${ }^{20}$ has emerged in the past several years as a handy tool that affords access to desired THPs. ${ }^{21}$ Throughout the past decade, our research group has taken advantage of the Prins cyclization to synthesize differently substituted six- and seven-membered oxa- and aza-heterocycles. ${ }^{22}$ Nevertheless, the application of the Prins cyclization to access challenging 2,3,4,5,6-pentasubstituted THPs has not been systematically studied. On one hand, there are only a few examples that have been part of methodological works that aimed to obtain THPs with a less populated substitution design. ${ }^{22 e, 23-26}$ On the other hand, Rychnovsky and co-workers have employed Prins cyclization to build pentasubstituted THPs to synthesize some natural products. $^{27-29}$

This absence of a general method encouraged us to propose a strategy based on the combination of the well-known Evans 
aldol addition, as a powerful tool to construct the necessary homoallylic alcohol, and the Prins cyclization to yield the target highly substituted THPs (Scheme 1). ${ }^{30}$ Thus, this Evans

Scheme 1. Retrosynthetic Analysis for Accessing 2,3,4,5,6Pentasubstituted THPs via an Evans Aldol-Prins Strategy

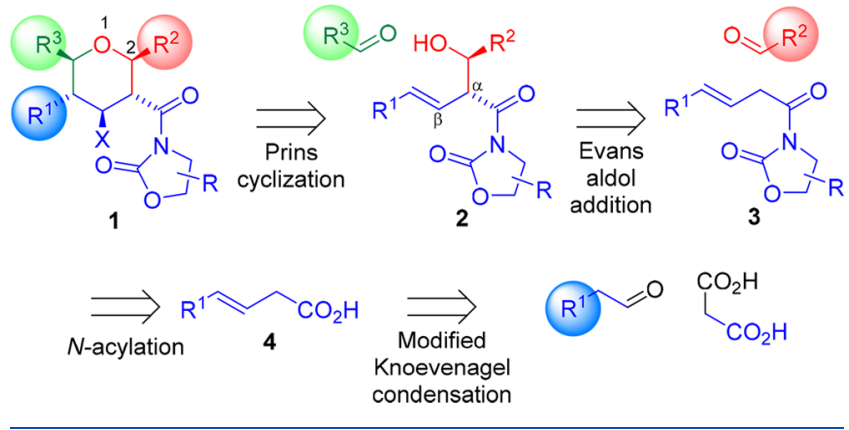

aldol-Prins (EAP) protocol suggests that a 2,3,4,5,6pentasubstituted THP 1 could be accessed via the Prins cyclization of an aldehyde $\mathrm{R}^{3} \mathrm{CHO}$ and syn-aldol $2 .{ }^{31} \beta, \gamma$ Unsaturated alcohols 2 bear an $\mathrm{N}$-acyl oxazolidin-2-one moiety at the $\alpha$ position. This auxiliary is the key for introduction of the stereochemistry into the aldol and, therefore, into the subsequent THP 1. Evans aldol addition was proposed as the diastereoselective pathway to obtain aldol 2 starting from a generic aldehyde $\mathrm{R}^{2} \mathrm{CHO}$ and $\beta, \gamma$-unsaturated $\mathrm{N}$-acyl oxazolidin-2-one 3 . $^{32}$ Compounds 3 may be prepared from the appropriate oxazolidin-2-one via an $N$-acylation of $\beta, \gamma$ - unsaturated carboxylic acids $4 .^{33}$ Several of these acids are commercially available, though they also can be readily synthesized via a modified Knoevenagel condensation starting from aldehydes $\mathrm{R}^{1} \mathrm{CH}_{2} \mathrm{CHO}$. ${ }^{34}$ Thus, the envisioned tactic should allow stereoselective access to an enormous degree of structural complexity by the consecutive combination of three different aldehydes in four reaction steps. Prior to the establishment of an asymmetric strategy, nonchiral oxazolidin-2-one was first selected as the Evans auxiliary to evaluate its influence in this unprecedented process. In this report, we expand the results previously published ${ }^{30}$ to exhaustively detail all the studies that led us to establish a protocol to yield 2,3,4,5,6-pentasubstituted THPs in a general and diastereoselective fashion. Special emphasis has been placed on the different reaction condition screenings and the identification of all the byproducts and minor stereoisomers associated with the EAP protocol. We have also delved into mechanistic studies, the enantiomeric approach, and the derivatization and biological evaluation of the novel family of compounds synthesized.

\section{RESULTS AND DISCUSSION}

As shown in Scheme 1, our synthetic approach first requires the preparation of a set of $N$-acyl oxazolidin-2-ones 3 employing carboxylic acids 4 as starting materials (Table 1 ). Acids $\mathbf{4 a - 4 c}$ were commercially available, whereas acids $\mathbf{4 d -}$ 4f were straightforwardly obtained via a solvent-free condensation/decarboxylation sequence. ${ }^{34}$ Acids 4 were differently activated prior to their subsequent treatment with the lithiated oxazolidin-2-one to yield 3. Both DMF/oxalyl

Table 1. Synthesis of the $N$-Acyl Oxazolidin-2-ones 3 and Aldols 2

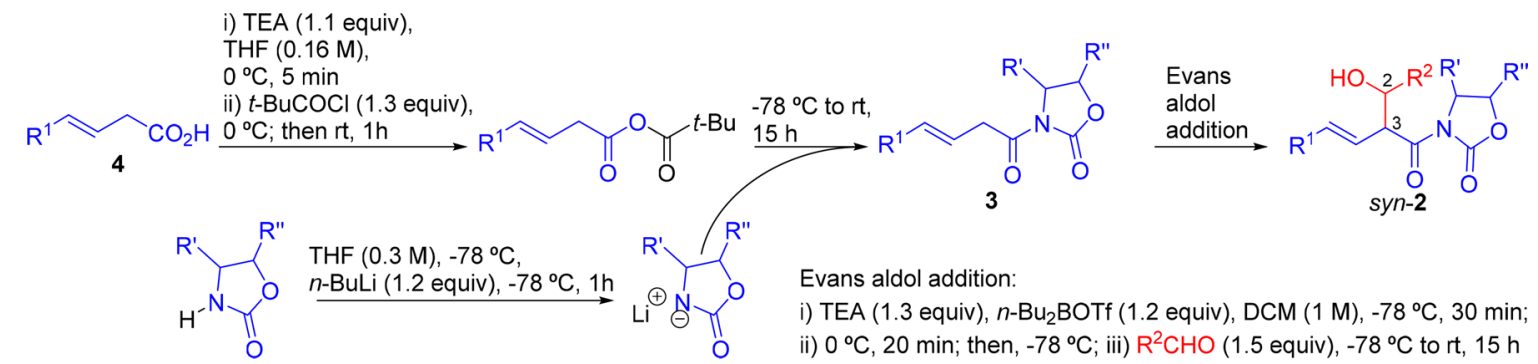

\begin{tabular}{|c|c|c|c|c|c|c|c|c|c|}
\hline \multicolumn{6}{|c|}{$N$-acylation } & \multicolumn{4}{|c|}{ Evans aldol addition } \\
\hline entry & acid & $\mathrm{R}^{1}$ & $\mathrm{R}^{\prime}$ & $\mathrm{R}^{\prime \prime}$ & $3(\%)$ & entry & 3 & $\mathrm{R}^{2}$ & $2(\%)^{c}$ \\
\hline 1 & $4 a$ & Et & $\mathrm{H}$ & $\mathrm{H}$ & $3 a(68)^{a}$ & 10 & $3 a$ & $i-\mathrm{Bu}$ & $\mathbf{2 a}(81)^{d}$ \\
\hline 2 & $4 b$ & $\mathrm{H}$ & $\mathrm{H}$ & $\mathrm{H}$ & $3 \mathbf{b}(39)^{b}$ & 11 & $3 a$ & $\mathrm{Me}$ & $2 b(75)$ \\
\hline 3 & $4 c$ & $(Z)-\mathrm{Me}$ & $\mathrm{H}$ & $\mathrm{H}$ & $3 c(75)$ & 12 & $3 a$ & $\mathrm{Bu}$ & $2 c(84)$ \\
\hline 4 & $4 d$ & $\mathrm{PhCH}_{2}$ & $\mathrm{H}$ & $\mathrm{H}$ & $3 d(60)$ & 13 & $3 a$ & $\mathrm{Ph}$ & $2 d(81)$ \\
\hline 5 & $4 e$ & $n$-pentyl & $\mathrm{H}$ & $\mathrm{H}$ & $3 e(90)$ & 14 & $3 a$ & $\mathrm{PhCH}_{2} \mathrm{CH}_{2}$ & $2 \mathrm{e}(81)$ \\
\hline 6 & $4 f$ & $\mathrm{BnOCH}_{2} \mathrm{CH}_{2}$ & $\mathrm{H}$ & $\mathrm{H}$ & $3 f(60)$ & 15 & $3 b$ & $\mathrm{Me}$ & $2 f(76)$ \\
\hline 7 & $4 a$ & Et & $(R)-\mathrm{Bn}$ & $\mathrm{H}$ & $3 g(90)$ & 16 & $3 c$ & $i-\mathrm{Bu}$ & $2 \mathrm{~g}(48)^{e}$ \\
\hline 8 & $4 a$ & Et & $(S)-i-\operatorname{Pr}$ & $\mathrm{H}$ & $3 h(83)$ & 17 & $3 a$ & 4-Br-Ph & $2 \mathrm{~h}(59)$ \\
\hline 9 & $4 a$ & Et & $(R)-\mathrm{Me}$ & $(S)-\mathrm{Ph}$ & $3 \mathbf{i}(87)$ & 18 & $3 g$ & $\mathrm{Me}$ & $2 \mathbf{i}(75)^{f}$ \\
\hline & & & & & & 19 & $3 g$ & $\mathrm{PhCH}_{2} \mathrm{CH}_{2}$ & $2 \mathbf{j}(60)^{f}$ \\
\hline & & & & & & 20 & $3 h$ & $\mathrm{Me}$ & $2 \mathbf{k}(86)^{g}$ \\
\hline & & & & & & 21 & $3 h$ & $\mathrm{PhCH}_{2} \mathrm{CH}_{2}$ & $21(69)^{g}$ \\
\hline & & & & & & 22 & $3 \mathbf{i}$ & $\mathrm{Bu}$ & $2 \mathbf{m}(64)^{f}$ \\
\hline
\end{tabular}

${ }^{a}$ For $3 \mathrm{a}, 7.7 \mathrm{~g}$ of product was obtained from $7 \mathrm{~g}$ of $4 \mathrm{a}^{b}{ }^{b}$ It was isolated as $54 \%$ of an inseparable $2.5: 1$ mixture of the desired product, $\beta, \gamma-3 \mathrm{~b}$, and its positional isomer, $E-\alpha, \beta-3 b$. ${ }^{c}$ Only syn-aldols were detected by ${ }^{1} \mathrm{H}$ NMR analysis of the reaction crudes, unless noted otherwise. ${ }^{d}$ Traces of its diastereoisomer, anti-2a, were also isolated when a non-properly stored $n$-Bu $\mathrm{B}_{2} \mathrm{BOTf}$ was employed. ${ }^{e 1} \mathrm{H}$ NMR analysis of the crude revealed that aldol $2 \mathrm{~g}$ was obtained as a 2:1 mixture of the $Z$ - and $E$-isomers due to an isomerization of the double bond, although the yield given corresponds exclusively to the desired $Z$-isomer. ${ }^{f} S, 3 R .{ }^{g} 2 R, 3 S$. 
Scheme 2. Fe-Based Prins Cyclization of Aldol 2a To Yield Unexpected Bicycle 5a

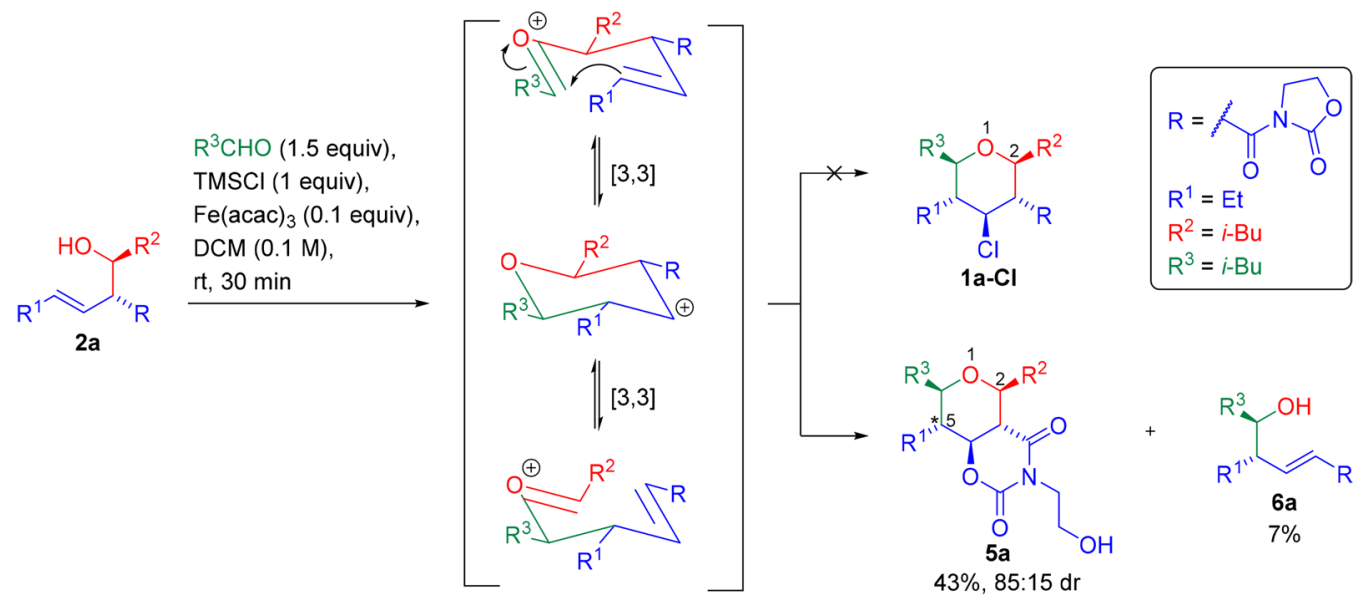

Table 2. Screening of Lewis Acids (LAs)

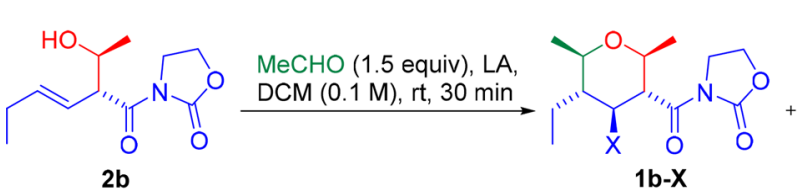<smiles>CC[C@H]1OC(=O)N(CCO)C(=O)[C@@H]2C(C)OC(C)C[C@@H]21</smiles><smiles>CC[C@H](C)/C=C/C(=O)N1CCOC1=O</smiles><smiles>[X]CCN1C(=O)O[C@H]2[C@@H](CC)[C@@H](C)O[C@H](C)[C@H]2C1=O</smiles>

\begin{tabular}{|c|c|c|c|c|c|c|c|}
\hline entry & LA & equiv & $\mathrm{X}$ & $\mathbf{1} \mathbf{b}-\mathbf{X}(\%)^{a}$ & $\mathbf{5 b}(\%)^{a}$ & $\mathbf{6 b}(\%)^{b}$ & $7 \mathbf{b}-\mathbf{X}(\%)^{a}$ \\
\hline 1 & $\mathrm{Fe}(\mathrm{acac})_{3} / \mathrm{TMSCl}$ & $0.1 / 1.5$ & - & - & 38 & 1 & - \\
\hline $2^{c}$ & $\mathrm{Fe}(\mathrm{acac})_{3} / \mathrm{TMSCl}$ & $0.5 / 1.5$ & - & - & 59 & - & - \\
\hline $3^{d}$ & $\mathrm{Fe}(\mathrm{acac})_{3} / \mathrm{TMSCl}$ & $0.02 / 1.5$ & - & - & 28 & 3 & - \\
\hline 4 & $\mathrm{FeCl}_{3}$ & 1.5 & - & - & 41 & 2 & - \\
\hline 5 & $\mathrm{InCl}_{3}$ & 1.5 & - & - & 61 & 15 & - \\
\hline 6 & $\mathrm{BF}_{3} \cdot \mathrm{THF}$ & 2.5 & - & - & 70 & 13 & - \\
\hline 7 & $\mathrm{BF}_{3} \cdot \mathrm{OEt}_{2}$ & 2.5 & - & - & 78 & - & - \\
\hline 8 & $\mathrm{BF}_{3} \cdot \mathrm{OEt}_{2}$ & 5 & - & - & 79 & - & - \\
\hline 9 & $\mathrm{BF}_{3} \cdot \mathrm{OEt}_{2}$ & 1 & - & - & 68 & 3 & - \\
\hline $10^{d}$ & $\mathrm{BF}_{3} \cdot \mathrm{OEt}_{2}$ & 0.05 & - & - & 58 & 7 & - \\
\hline $11^{d, e}$ & $\mathrm{BF}_{3} \cdot \mathrm{OEt}_{2} / \mathrm{TMSCl}$ & $0.05 / 2.5$ & $\mathrm{Cl}$ & - & - & 10 & 46 \\
\hline $12^{f}$ & $\mathrm{BF}_{3} \cdot \mathrm{OEt}_{2} / \mathrm{TMSCl}$ & $0.5 / 2.5$ & $\mathrm{Cl}$ & - & - & 6 & 67 \\
\hline $13^{g}$ & TMSCl & 2.5 & $\mathrm{Cl}$ & - & - & 6 & $45^{b}$ \\
\hline $14^{h}$ & TMSI & 2.5 & I & 17 & - & - & 58 \\
\hline $15^{i}$ & TMSBr & 2.5 & $\mathrm{Br}$ & 30 & - & - & 46 \\
\hline 16 & $\mathrm{FeBr}_{3}$ & 2.5 & $\mathrm{Br}$ & 23 & 34 & 9 & - \\
\hline
\end{tabular}

${ }^{a}$ Isolated yield, unless noted otherwise; $>95: 5 \mathrm{dr}$ (determined by ${ }^{1} \mathrm{H}$ NMR spectroscopy). ${ }^{b}$ Calculated by ${ }^{1} \mathrm{H}$ NMR spectroscopy. ${ }^{c}$ The

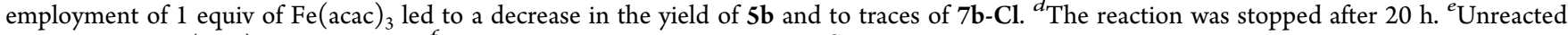
starting material (44\%) was recovered. ${ }^{f_{\text {The }}}$ reaction was stopped after $2 \mathrm{~h} .{ }^{g}$ The reaction was stopped after $44 \mathrm{~h}$, and $49 \%$ of unreacted starting material was found. ${ }^{h}$ The reaction was stopped after $20 \mathrm{~min} .{ }^{i}$ The reaction was stopped after $3 \mathrm{~h}$.

chloride $^{35}$ and DCC/DMAP ${ }^{36}$ systems proved to be efficient, but we eventually selected TEA and pivaloyl chloride ${ }^{33}$ as reagents because of their compatibility with the multigram synthesis of 3a (Table 1, entry 1). With these conditions in hand, nonchiral $N$-acyl oxazolidin-2-ones $\mathbf{3 b}-\mathbf{3} \mathbf{f}$ (entries $2-6$, respectively) and chiral $N$-acyl oxazolidin-2-ones $3 \mathbf{g}-\mathbf{3 i}$ (entries 7-9, respectively) were efficiently synthesized with yields ranging from 60 to $90 \%$, except when the starting acid bore a terminal double bond $\left(4 \mathbf{b}\right.$, where $\left.R^{1}=H\right)$, because undesired $E-\alpha, \beta-3 \mathbf{b}$ was also obtained as a consequence of the isomerization of the double bond (entry 2). ${ }^{37} \mathrm{~N}$-Acyl oxazolidin-2-ones $3 \mathbf{a}-\mathbf{3} \mathbf{c}$ were submitted to the Evans protocol to gain access to various syn-aldols 2 with good yields and showed excellent tolerance to aromatic groups and both linear and branched aliphatic chains (entries 10-17). ${ }^{32}$ Similarly, chiral aldols $\mathbf{2 i}-\mathbf{2 m}$ were obtained from $\mathrm{N}$-acyl oxazolidin-2ones $3 \mathbf{g}-\mathbf{3 i}$ (entries 18-22). As expected, in all cases, synaldols were exclusively obtained except when a non-properly stored $n-\mathrm{Bu}_{2} \mathrm{BOTf}$ solution was employed (entry 10 ), because the diastereoselectivity of the aldol addition may be sensitive to the concentration of that reagent. ${ }^{38}$ Isolation of traces of anti2a invited us to try the efficient and selective access to that aldol, although employment of the methods previously published by Evans ${ }^{39}$ and Hoye ${ }^{40}$ was unsuccessful. Additionally, when $\mathrm{N}$-acyl oxazolidin-2-one 3c, bearing a double bond with a $Z$ geometry, was selected as the starting material, a partial isomerization of the double bond was observed in the final product, leading to the desired $\mathbf{2} \mathbf{g}$ with a moderate yield (entry 16$)$. 
With aldols 2 in hand, we decided to test the Prins cyclization conditions previously optimized in our research group, employing the $\mathrm{Fe}(\mathrm{acac})_{3} / \mathrm{TMSCl}$ system as a promoter. $^{22 \mathrm{~d}}$ 4-Chloro-THP $\mathbf{1 a - C l}$ was selected as a target molecule, and aldol $\mathbf{2 a}$ and isovaleraldehyde were selected as starting materials (Scheme 2). The presence of each $i$-Bu at positions 2 and 6 of the ring should avoid the production of undesired THPs as a consequence of the side chain exchange due to the 2-oxonia-Cope rearrangement, a [3,3]-sigmatropic rearrangement concomitant to the Prins cyclization. ${ }^{41}$ Aldol 2a yielded two products after $30 \mathrm{~min}$, one of them more nonpolar than the substrate and the other more polar and UV-visible. With regard to the nonpolar product, ${ }^{1} \mathrm{H}$ NMR analysis confirmed the presence of a THP ring with the expected side chains. However, mass analysis revealed that the molecule did not include a chlorine atom. Fortunately, X-ray crystallography unambiguously determined that the product was $\mathbf{5 a}$, in which the THP ring appeared to be fused to a 1,3-oxazinane-2,4dione ring (Scheme 2). Thus, bicycle 5a was obtained as a mixture of two diastereoisomers $(85: 15 \mathrm{dr})$ in $43 \%$ total yield. The X-ray analysis, together with the $J$ coupling of $>9 \mathrm{~Hz}^{42}$ and GOESY experiments, ${ }^{43}$ allowed us to establish an all-trans configuration in the major diastereoisomer. GOESY experiments also revealed that the minor diastereoisomer was the $\mathrm{C}_{5}$ epimer (Scheme 2). On the other hand, the polar product was obtained in $7 \%$ yield and identified as alcohol 6a, a skeletal isomer of aldol $2 \mathrm{a}$, obtained because of the 2-oxonia-Cope rearrangement (Scheme 2). ${ }^{41}$ Once the products of the reaction were identified, we directed our attention to the unprecedented synthesis of bicycle 5. Rearrangements of $\mathrm{N}$ acyl oxazolidin-2-ones to yield these kinds of heterocycles had been previously reported, ${ }^{44}$ though, to the best of our knowledge, this was the first example in which the 1,3oxazinane-2,4-dione ring was fused with a THP. Additionally, it should be remarked that both heterocycles usually are related with varied biological activities such as antiepileptic, ${ }^{45}$ analgesic, $^{46}$ and antiproliferative ${ }^{11}$ activities. A synergistic biological activity might be expected from these structures. Despite its unexpected bicyclic structure, compound $\mathbf{5 a}$ is a 2,3,4,5,6-pentasubstituted THP, so it consequently satisfies our initial synthetic goal (Scheme 1). Additionally, the uniqueness of this bicyclic core encouraged us to delve into the synthesis of these kinds of compounds.

We first screened a series of Lewis acids (LAs) to pursue better yields of bicycles $\mathbf{5}$. We chose alcohol $\mathbf{2} \mathbf{b}$ and $\mathrm{MeCHO}$ as the most simple starting materials to access a 2,3,4,5,6pentasubstituted THP (Table 2). First, those reagents were submitted to the previously published conditions with the $\mathrm{Fe}(\mathrm{acac})_{3} / \mathrm{TMSCl}$ system (Scheme 2), ${ }^{22 \mathrm{~d}}$ yielding expected bicycle $\mathbf{5 b}$ only as a diastereoisomer with a yield (38\%) similar to that of $\mathbf{5 a}(43 \%)$, together with traces of undesired rearrangement isomer $\mathbf{6} \mathbf{b}$ (Table 2, entry 1$)$. A higher level of $\mathrm{Fe}(\mathrm{acac})_{3}$ improved the yield of $\mathbf{5 b}$ and avoided the formation of $\mathbf{6 b}$ (entry 2 vs entry 3 ). Nevertheless, when $\mathrm{Fe}(\mathrm{acac})_{3}$ and TMSCl were employed separately as catalysts, the cyclization did not occur after reaction for $5 \mathrm{~h}$. The yield of $\mathbf{5 b}$ did not improve when an excess of $\mathrm{FeCl}_{3}$ was employed as an alternative source of $\mathrm{Fe}$ (III) (entry 4), although a suprastoichiometric quantity of $\mathrm{InCl}_{3}$ led to $\mathbf{5 b}$ with an interesting $61 \%$ yield (entry 5 ). When 0.1 equiv of those iron and indium compounds was tested, unaltered starting material was recovered. Other promoters were tested ${ }^{47}$ until we discovered that $\mathrm{BF}_{3} \cdot \mathrm{THF}$ allowed the synthesis of $\mathbf{5 b}$ with a remarkable
$70 \%$ yield, although with a higher proportion of $\mathbf{6 b}$ (entry 6). To our delight, a better yield was obtained when $\mathrm{BF}_{3} \cdot \mathrm{OEt}_{2}$ was chosen as the LA, and $\mathbf{6 b}$ was not detected (entry 7 ). Almost the same yield was obtained when a larger amount of $\mathrm{BF}_{3} \cdot \mathrm{OEt}_{2}$ was employed (entry 8), but when the amount of the LA was progressively reduced, the yield of $\mathbf{5 b}$ decreased in favor of an increase in the yield of nondesired $\mathbf{6 b}$ and a longer reaction time (entries 9 and 10). Then, we decided to evaluate the combined effect of $\mathrm{BF}_{3} \cdot \mathrm{OEt}_{2}$ with $\mathrm{TMSCl}$ as the promoter of the EAP cyclization (entries 11 and 12$)$. We repeated the reaction shown in entry $10\left(0.05\right.$ equiv of $\left.\mathrm{BF}_{3} \cdot \mathrm{OEt}_{2}\right)$, including 2.5 equiv of $\mathrm{TMSCl}$ in the set of reagents. Surprisingly, under these conditions, the main product of the reaction was halogenated bicycle $\mathbf{7 b}-\mathbf{C l}$ instead of bicycle $\mathbf{5 b}$ (entry 11). Additionally, rearranged alcohol $\mathbf{6 b}$ was also obtained together with part of the unreacted starting material. When the amount of $\mathrm{BF}_{3} \cdot \mathrm{OEt}_{2}$ was increased to 0.5 equiv, the starting aldol was consumed, yielding $67 \% \mathbf{7 b}-\mathbf{C l}$ along with traces of $\mathbf{6 b}$ (entry 12). By contrast, when 2.5 equiv of $\mathrm{TMSCl}$ was employed as the sole promoter, a practically equimolar mixture of halogenated product $\mathbf{7 b}-\mathbf{C l}$ and starting material was detected after $44 \mathrm{~h}$ (entry 13). Next, we decided to check the influence of the halogen on this process. When TMSI was employed as the promoter, full conversion of aldol $\mathbf{2} \mathbf{b}$ was observed after only $10 \mathrm{~min}$, and two products were identified (entry 14): expected halogenated bicycle $7 \mathbf{b}$-I (58\%) and 4-iodine-THP 1b-I (17\%), with the originally pursued structure (Scheme 1). In the same vein, TMSBr allowed access to $7 \mathbf{b}-\mathbf{B r}(46 \%)$ and $\mathbf{1 b}-\mathbf{B r}(30 \%)$ after reaction for $3 \mathrm{~h}$ (entry 15$).{ }^{48}$ We eventually tested the EAP cyclization employing $\mathrm{FeBr}_{3}$ both as a $\mathrm{LA}$ and as a bromide source. The reaction was complete after $30 \mathrm{~min}$, yielding 4-bromo-THP $\mathbf{1 b}$-Br (23\%), hydroxylated bicycle $\mathbf{5 b}$ $(34 \%)$, and rearranged alcohol $\mathbf{6 b}(9 \%)$, but no traces of halogenated bicycle $7 \mathbf{b}$-Br were detected (entry 16 ).

Once the benefits of $\mathrm{BF}_{3} \cdot \mathrm{OEt}_{2}$ as promoter of the EAP cyclization had been verified, the effect of the solvent was studied (Table 3). Bicycle $\mathbf{5 c}$ was obtained as a sole diastereoisomer in $66 \%$ yield from aldol $2 c$ and $n$-pentanal employing DCM as the solvent (entry 1 ). Second, the reaction was repeated several times replacing DCM with solvents such

Table 3. Screening of Solvents
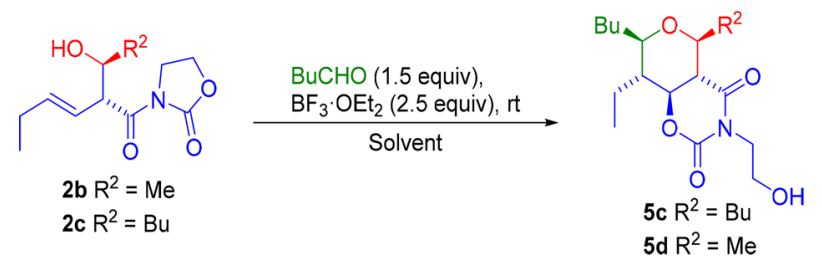

$\begin{array}{cc}\text { entry } & \text { al } \\ 1 & 2 c \\ 2 & 2 c \\ 3 & 2 c \\ 4 & 2 c \\ 5 & 2 c \\ 6^{b} & 2 c \\ 7 & 2 b \\ 8 & 2 b \\ 9 & 2 b\end{array}$

aldol
$2 \mathrm{c}$
$2 \mathrm{c}$
$2 \mathrm{c}$
$2 \mathrm{c}$
$2 \mathrm{c}$
$2 \mathrm{c}$
$2 \mathrm{~b}$
$2 \mathrm{~b}$
$2 \mathrm{~b}$

$\quad$ solvent
DCM
acetonitrile
benzene
toluene
$\mathrm{Et}_{2} \mathrm{O}$
acetic acid
$\mathrm{DCM}^{\mathrm{CHCl}_{3}}$
$n$-hexane

$\begin{array}{lc}\text { bicycle } & \text { yield }(\%)^{a} \\ \text { 5c } & 66 \\ \text { 5c } & 55 \\ \text { 5c } & 52 \\ \text { 5c } & 43 \\ \text { 5c } & 21 \\ \text { 5c-Ac } & 71 \\ \text { 5d } & 69 \\ \text { 5d } & 50 \\ \text { 5d } & 31\end{array}$

${ }^{a}$ Isolated yield; >95:5 dr (determined by ${ }^{1} \mathrm{H}$ NMR spectroscopy).

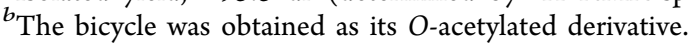




\section{Scheme 3. Synthesis of Bicycles with Different Stereochemical Patterns}
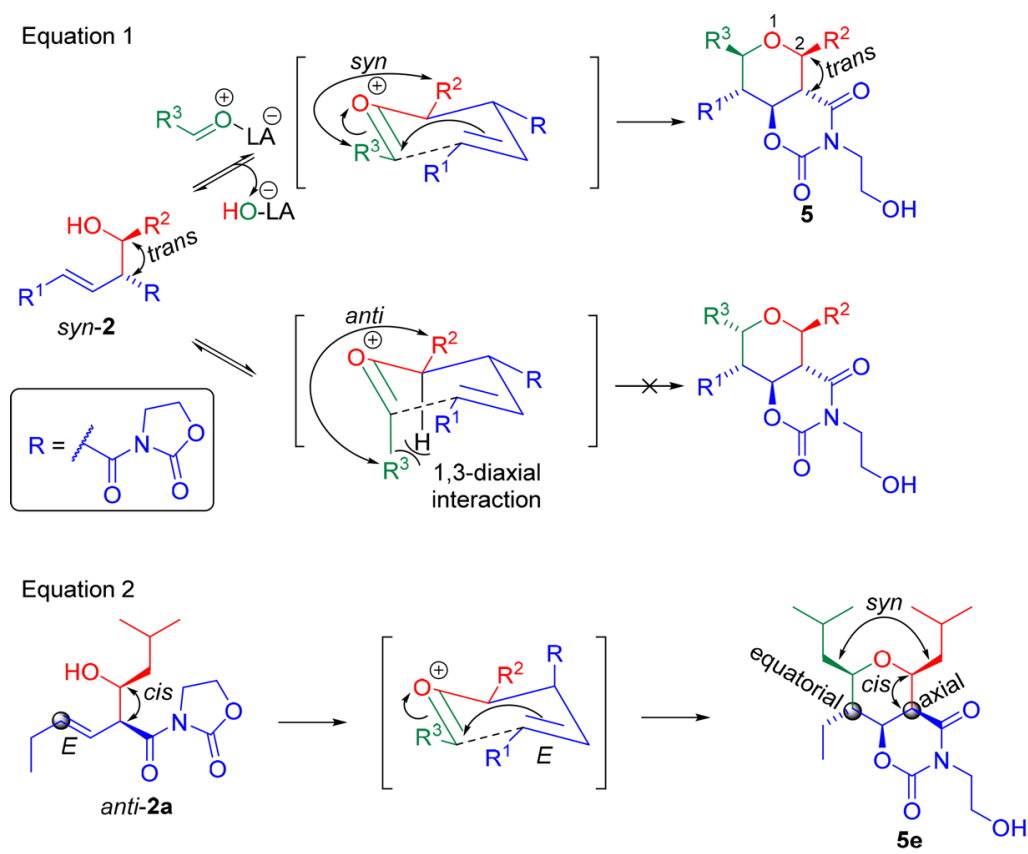

Method $\mathrm{A}: i$ - $\mathrm{BuCHO}$ (1.5 equiv), $\mathrm{FeCl}_{3}$ (1 equiv)

DCM (0.1 M), rt, $30 \mathrm{~min}, 38 \%, 70: 22: 8 \mathrm{dr}$

Method $\mathrm{B}: i$-BuCHO (1.5 equiv), $\mathrm{BF}_{3} \cdot \mathrm{OEt}_{2}$ (2.5 equiv)

DCM (0.1 M), rt, $30 \mathrm{~min}, 49 \%, 75: 19: 6 \mathrm{dr}$

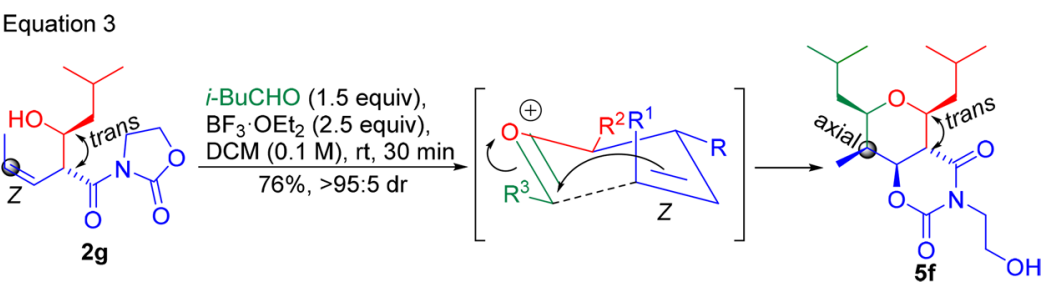

as acetonitrile, benzene, and toluene, although the yield did not improve (entries 2-4, respectively). The crude mixtures of those reactions were thoroughly studied by ${ }^{1} \mathrm{H}$ NMR to check that no byproducts associated with competitive Prins-Ritter ${ }^{49}$ or Prins-Friedel-Crafts ${ }^{50}$ processes (both associated with nucleophilic solvents) were obtained. The selection of $\mathrm{Et}_{2} \mathrm{O}$ as the solvent led to the poorest yield (entry 5). Interestingly, when acetic acid was employed as the solvent, bicycle $\mathbf{5 c}$ was obtained as its $\mathrm{O}$-acetylated derivative (entry 6). However, if the acetic acid remained as the solvent and $\mathrm{BF}_{3} \cdot \mathrm{OEt}_{2}$ was omitted as the promoter, the reaction did not take place. The Prins cyclization between aldol $\mathbf{2} \mathbf{b}$ and $n$-pentanal was also tested employing DCM as the solvent to access bicycle $\mathbf{5 d}$ in $69 \%$ yield (entry 7 ). There was a drop in the yield when the reaction was repeated in the presence of $\mathrm{CHCl}_{3}$ or $n$-hexane as the solvent (entry 8 or 9 , respectively).

The studies described above allowed us to conclude that the optimized conditions for the EAP cyclization imply the use of 2.5 equiv of $\mathrm{BF}_{3} \cdot \mathrm{OEt}_{2}$ and $\mathrm{DCM}$ as the solvent at $\mathrm{rt}^{51}$ This EAP cyclization has proven to be a diastereoselective method for synthesizing 2,3,4,5,6-pentasubstituted THPs with an alltrans stereochemistry, placing their substituents in equatorial positions. The substituents at $\mathrm{C}_{2}$ and $\mathrm{C}_{6}$ of the THP adopt a preferred syn stereochemistry to minimize the 1,3-diaxial interaction in the chairlike transition state (Scheme 3, eq 1)..$^{52}$ The position of the oxygen atom linked to $\mathrm{C}_{4}$ is a consequence of the position adopted by the oxazolidin-2-one in the transition state, as will be discussed in the mechanistic section (Scheme 7). With regard to the stereochemistry of $C_{3}$ and $C_{5}$, it is controlled by the stereochemistry of the starting alcohol. On one hand, the trans disposition of the substituents of the syn-aldol ${ }^{31}$ leads to the trans orientation of the substituents at $\mathrm{C}_{2}$ and $\mathrm{C}_{3}$ (eqs 1 and 3 ); by contrast, an anti-aldol should develop a cis orientation of those substituents (eq 2). On the other hand, an $E$ geometry of the olefin is conducted to the equatorial position of the substituent at $C_{5}$ (eqs 1 and 2), whereas a $Z$ geometry should favor the axial position (eq 3). A further mechanistic discussion appears in Scheme $7 .^{26}$ As we had obtained a small amount of anti-2a (Table 1, entry 7), we decided to evaluate it in the EAP cyclization to synthesize bicycle $5 \mathrm{e}$ with the substituent at $\mathrm{C}_{3}$ in an axial position (Scheme 3, eq 2). Thus, anti-2a was subjected to each reaction employing $\mathrm{FeCl}_{3}\left(\right.$ method A) and $\mathrm{BF}_{3} \cdot \mathrm{OEt}_{2}($ method $\mathrm{B})$ as Lewis acids. In both cases, the main product was expected bicycle 5e, whose relative stereochemistry was confirmed by GOESY analysis. However, the employment of anti-2a as the starting material yielded the synthesis of two undesired minor diastereoisomers in a 3:1 proportion: the main one was identified as all-trans bicycle $\mathbf{5 a}$, whereas the other was its $\mathrm{C}_{5}$ epimer. ${ }^{53}$ When syn-aldol $\mathbf{2 g}$, bearing a $Z$-olefin, was submitted to the optimized EAP cyclization conditions, desired bicycle $\mathbf{5 f}$ was diastereoselectively obtained in $76 \%$ yield (Scheme 3 , eq 3). As bicycles $\mathbf{5 e}$ and $\mathbf{5 f}$, bearing axial substituents, can be accessed via EAP cyclization, it therefore constitutes an 


\section{Table 4. Synthesis of Differently Substituted THPs}

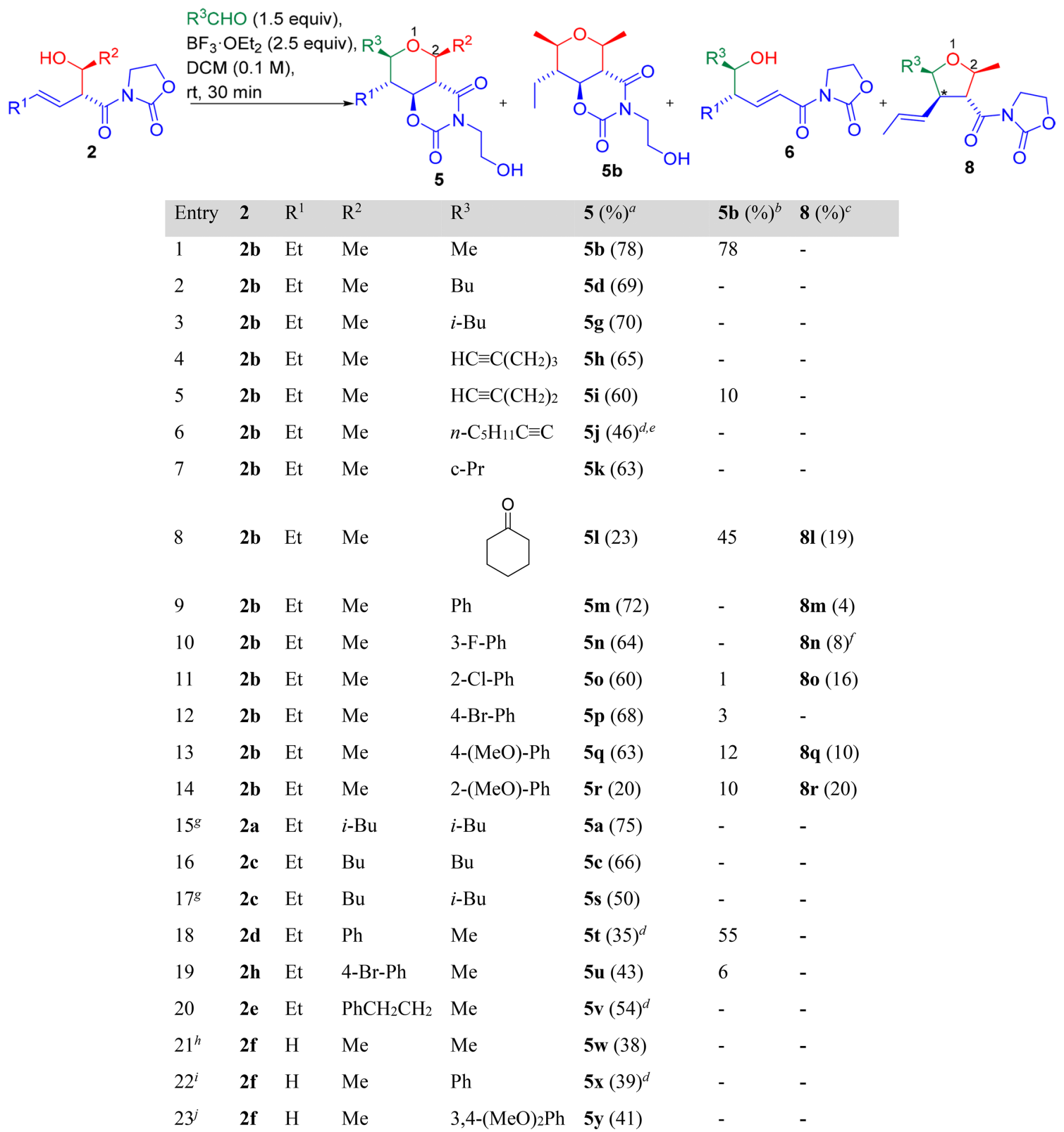

${ }^{a}$ Isolated yield; >95:5 dr, unless noted otherwise (determined by ${ }^{1} \mathrm{H}$ NMR spectroscopy). ${ }^{b}$ Calculated by ${ }^{1} \mathrm{H}$ NMR spectroscopy, except in entry 1 , where $\mathbf{5 b}$ is the expected product. ${ }^{c}$ Isolated yield; 80:20 dr, unless noted otherwise (determined by ${ }^{1} \mathrm{H}$ NMR spectroscopy). ${ }^{d} \mathrm{~A} 90: 10 \mathrm{dr}$ (determined by ${ }^{1} \mathrm{H}$ NMR spectroscopy). ${ }^{e} \mathrm{~A} 39 \%$ yield was obtained when aldehyde was employed protected as diethylacetal. ${ }^{f}$ An $85: 15$ dr (determined by ${ }^{1} \mathrm{H}$ NMR spectroscopy). ${ }^{g}$ Reaction performed at $0{ }^{\circ} \mathrm{C}$. ${ }^{h}$ Forty percent of $6 \mathbf{b}$ was also isolated; when $n$-hexane (0.05 M) was employed as the solvent, $50 \%$ of $\mathbf{5 w}$ and $30 \%$ of $\mathbf{6} \mathbf{b}$ were isolated. ${ }^{i} \mathbf{6 x}(35 \%)$ and $\mathbf{5 w}(14 \%,>95: 5 \mathrm{dr})$ were calculated by ${ }^{1} \mathrm{H}$ NMR spectroscopy. ${ }^{j} \mathbf{6 y}$ $(10 \%)$ and $5 \mathbf{w}(14 \%,>95: 5 \mathrm{dr})$ were calculated by ${ }^{1} \mathrm{H}$ NMR spectroscopy.

interesting tool for accessing the core of natural products such as kendomycin or phorboxazols (Figure 1).

Afterward, we selected syn-aldol $\mathbf{2} \mathbf{b}$ as the starting material to check the robustness of the optimized conditions $\left(\mathrm{BF}_{3}\right.$. $\left.\mathrm{OEt}_{2}, \mathrm{DCM}, \mathrm{rt}\right)$ by broadening the scope of the aldehydes (Table 4). Entries 1 and 2 show the previously described syntheses of bicycles $\mathbf{5 b}$ (Table 2, entry 7) and $\mathbf{5 d}$ (Table 3, entry 7), respectively. Besides these linear chains, the bulkier $i$ $\mathrm{Bu}$ was successfully introduced at the $\mathrm{C}_{6}$ position of the THP $\mathbf{5 g}$ in $70 \%$ yield (entry 3 ). Hex-5-ynal and pent-4-ynal were synthesized through a PCC-mediated oxidation of the corresponding commercial alcohols, ${ }^{54}$ and they were employed in the Prins cyclization without further purification, yielding bicycles $\mathbf{5 h}$ and $\mathbf{5 i}$, respectively (entries 4 and 5 , respectively). Together with $\mathbf{5 i}$, a small amount of bicycle $\mathbf{5 b}$ was detected because of the release of $\mathrm{MeCHO}$ to the medium as result of the 2-oxonia-Cope rearrangement. ${ }^{41} \mathrm{We}$ also chose oct-2-ynal to test an $\alpha, \beta$-unsaturated aldehyde, and bicycle $5 \mathbf{j}$ was obtained in $46 \%$ yield (entry 6 ). It should be noted that oct-2ynal protected as its diethylacetal led to the same product with a slightly inferior 39\% yield. Cyclopropanecarbaldehyde is an apparently problematic aldehyde because of the presence of an acid-sensitive motif, ${ }^{55}$ but to our delight, it reacted properly to give 5k in 63\% yield (entry 7 ). Cyclohexanone was also tested as a carbonylic compound in the EAP cyclization to study the production of spirotetrahydropyrans, ${ }^{56}$ providing 51 in $23 \%$ 
Scheme 4. Two-Step EAP Cyclization versus One-Pot EAP Cyclization

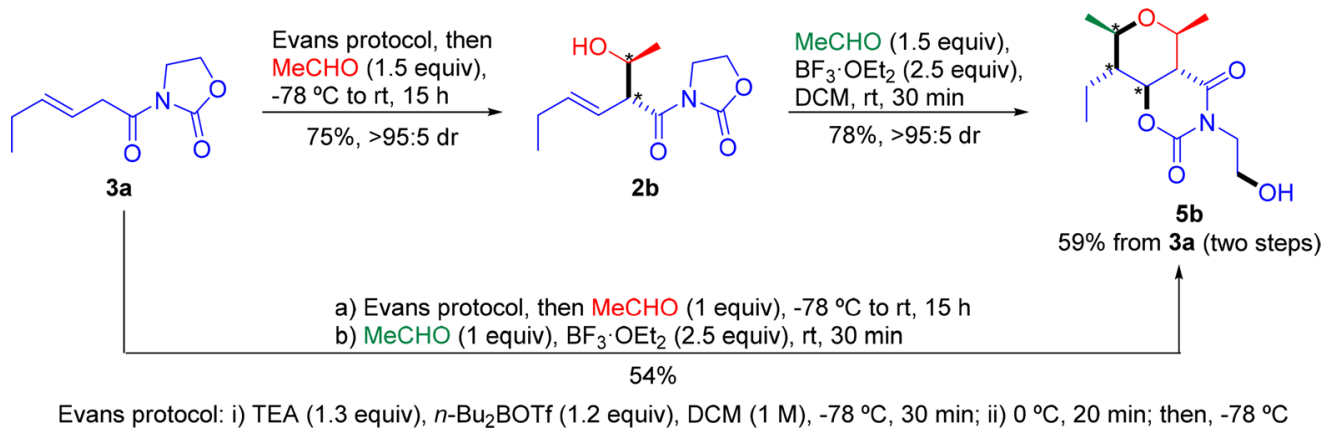

Table 5. Synthesis of 2,3,4,5,6-Pentasubstituted THPs via the One-Pot EAP Cyclization

\begin{tabular}{|c|c|c|c|c|c|c|c|}
\hline \multirow[b]{2}{*}{ entry } & \multirow[b]{2}{*}{ substrate } & $\pi^{N} \pi$ & \multicolumn{3}{|c|}{$\begin{array}{l}\text { a) i) TEA ( } 1.3 \text { equiv), } n-\mathrm{Bu}_{2} \mathrm{BOTf} \text { ( } 1.2 \text { equiv), } \\
\mathrm{DCM}(1 \mathrm{M}),-78^{\circ} \mathrm{C}, 30 \mathrm{~min} \\
\text { ii) } 0^{\circ} \mathrm{C}, 20 \mathrm{~min} \text {; then, }-78^{\circ} \mathrm{C} \\
\text { iii) } \mathrm{R}^{2} \mathrm{CHO}\left(1 \text { equiv), }-78^{\circ} \mathrm{C} \text { to } \mathrm{rt}, 15 \mathrm{~h}\right. \\
\text { b) } \mathrm{R}^{3} \mathrm{CHO}\left(1 \text { equiv), } \mathrm{BF}_{3} \cdot \mathrm{OEt}_{2}(2.5 \text { equiv), rt, } 30 \mathrm{~min}\right.\end{array}$} & \multirow{2}{*}{$\begin{array}{c}\mathrm{OH} \\
\text { vield }(\%)^{a}\end{array}$} & \multirow[b]{2}{*}{ yield $_{\mathrm{av}}(\%)^{b}$} \\
\hline & & $\mathrm{R}^{1}$ & $\mathrm{R}^{2}$ & $\mathrm{R}^{3}$ & bicycle & & \\
\hline 1 & $3 a$ & Et & $\mathrm{Me}$ & $\mathrm{Me}$ & $5 b$ & 54 & 88 \\
\hline 2 & $3 a$ & Et & 4-Br-Ph & $\mathrm{Me}$ & $5 \mathbf{u}$ & $22^{c}$ & 74 \\
\hline 3 & $3 a$ & Et & $n-\mathrm{C}_{13} \mathrm{H}_{27}$ & $\mathrm{Me}$ & $5 z$ & 41 & 84 \\
\hline 4 & $3 a$ & Et & $i-\mathrm{Bu}$ & $\mathrm{Me}$ & 5 aa & 42 & 84 \\
\hline 5 & $3 a$ & Et & $\mathrm{Bu}$ & $\mathrm{Ph}$ & $5 \mathrm{ab}$ & $31^{d}$ & 79 \\
\hline 6 & $3 a$ & Et & $i-\mathrm{Bu}$ & $i-\mathrm{Bu}$ & $5 a$ & $60^{e}$ & 90 \\
\hline 7 & $3 d$ & $\mathrm{PhCH}_{2}$ & $\mathrm{Bu}$ & $\mathrm{Bu}$ & $5 \mathrm{ac}$ & 32 & 80 \\
\hline 8 & $3 e$ & $n$-pentyl & $\mathrm{Me}$ & $\mathrm{Bu}$ & 5 ad & 31 & 79 \\
\hline 9 & $3 e$ & $n$-pentyl & $\mathrm{Bu}$ & $\mathrm{Me}$ & $5 a e$ & 30 & 79 \\
\hline 10 & $3 f$ & $\mathrm{BnOCH}_{2}$ & $\mathrm{Me}$ & $\mathrm{Me}$ & 5 af & $54^{f}$ & 88 \\
\hline
\end{tabular}

${ }^{a}$ Isolated yield; >95:5 dr, unless noted otherwise (determined by ${ }^{1} \mathrm{H}$ NMR spectroscopy). ${ }^{b}$ Average yield of each of the five new $\sigma$ bonds generated during the one-pot EAP cyclization. ${ }^{c} 5 \mathbf{b}$ was also isolated (4\%, >95:5 dr). ${ }^{d} \mathrm{~A}$ 90:10 dr (determined by ${ }^{1} \mathrm{H}$ NMR spectroscopy). ${ }^{e} \mathrm{~A}$ total of $5.3 \mathrm{~g}$ was obtained with an 85:15 dr. ${ }^{f}$ Obtained as a 1.3:1 benzylated/nonbenzylated THP mixture.

yield, although with excellent diastereoselectivity (entry 8 ). The low yield is a consequence of the influence of the 2oxonia-Cope rearrangement, which leads to bicycle $\mathbf{5 b}$ as the main product. Additionally, 2,3,4,5,5-pentasubstituted tetrahydrofuran (THF) 81 was isolated as a 4:1 mixture of the epimers at $\mathrm{C}_{4}$ with a $19 \%$ yield. It is proposed that THF $\mathbf{8 1}$ is a consequence of a 5-exo-trig attack of the olefin on the oxocarbenium ion, ${ }^{57}$ instead of the 6-endo-trig attack that is conducive to bicycle 5 . Afterward, benzaldehyde (entry 9) and several electron-poor aromatic aldehydes (entries 10-12) were evaluated. An electron-rich aromatic aldehyde carrying a $\mathrm{MeO}$ group in the para position also provided a similar good yield (entry 13), though a drop in the yield was observed when the same donor group was located in the orto position (entry 14). ${ }^{58}$ As seen when cyclohexanone was employed as the carbonylic compound (entry 8), corresponding 2,3,4,5tetrasubstituted THFs 8 were generally identified (entries 911,13 , and 14), unlike the results when aliphatic aldehydes were used as starting materials.

Once the effectiveness of the Prins cyclization between the simplest secondary aldol $\mathbf{2 b}\left(\mathrm{R}^{2}=\mathrm{Me}\right)$ and several aldehydes $\left(\mathrm{R}^{3} \mathrm{CHO}\right)$ was demonstrated, we decided to modify both substituents $R^{2}$ and $R^{3}$ (Table 5). As illustrated in entry 15 , when $\mathrm{R}^{2}=\mathrm{R}^{3}=i$ - $\mathrm{Bu}$, bicycle $\mathbf{5}$ a was efficiently synthesized as the sole diastereoisomer by performing the reaction at $0{ }^{\circ} \mathrm{C}$ (traces of the $\mathrm{C}_{5}$ epimer were obtained when the reaction was performed at rt). Entry 16 shows the previously discussed synthesis of bicycle 5c (Table 3, entry 1). When the synthesis of bicycle 5s was addressed, it was essential to perform the reaction at $0{ }^{\circ} \mathrm{C}$ to avoid obtaining traces of bicycles $\mathbf{5 a}$ and $\mathbf{5 c}$, as a consequence of the processes associated with the 2-oxoniaCope rearrangement (entry 17). ${ }^{41}$ However, when $\mathrm{MeCHO}$ was combined with $2 d\left(R^{2}=P h\right)$, the presence of an aromatic group directly attached to the hydroxy group of the aldol led us inevitably to a mixture of desired bicycle 5t (35\%) and side chain-exchanged byproduct $\mathbf{5 b}(55 \%)$ as the main product (entry 18). This phenomenon can be explained considering that if $\mathrm{R}^{2}$ is an aromatic group, it stabilizes by resonance the intermediate oxocarbenium obtained after the 2-oxonia-Cope rearrangement. ${ }^{59}$ As expected, the presence of an electronwithdrawing group in the aromatic moiety of aldol $2 \mathrm{~h}\left(\mathrm{R}^{2}=4\right.$ $\mathrm{Br}-\mathrm{Ph}$ ) led to a decrease in the yield of undesired byproduct $\mathbf{5 b}$ and yielded bicycle $\mathbf{5 u}$ diastereoselectively (entry 19). ${ }^{60,61}$ The presence of a two-unit methylene bridge between the aromatic and hydroxy groups of aldol $2 \mathrm{e}\left(\mathrm{R}^{2}=\mathrm{PhCH}_{2} \mathrm{CH}_{2}\right)$ allowed likewise an improvement in the yield in the synthesis of bicycle 5v (entry 20). Then we tested the EAP cyclization employing aldol $2 \mathrm{f}\left(\mathrm{R}^{1}=\mathrm{H}\right)$ as the starting material. When it was combined with $\mathrm{MeCHO}$, the absence of an aliphatic chain attached to the olefin led to bicycle $5 \mathbf{w}$ in $38 \%$ yield (entry 21 ), 
Scheme 5. Influence of the Nature of the Substituent Directly Attached to the Carbonyl Group
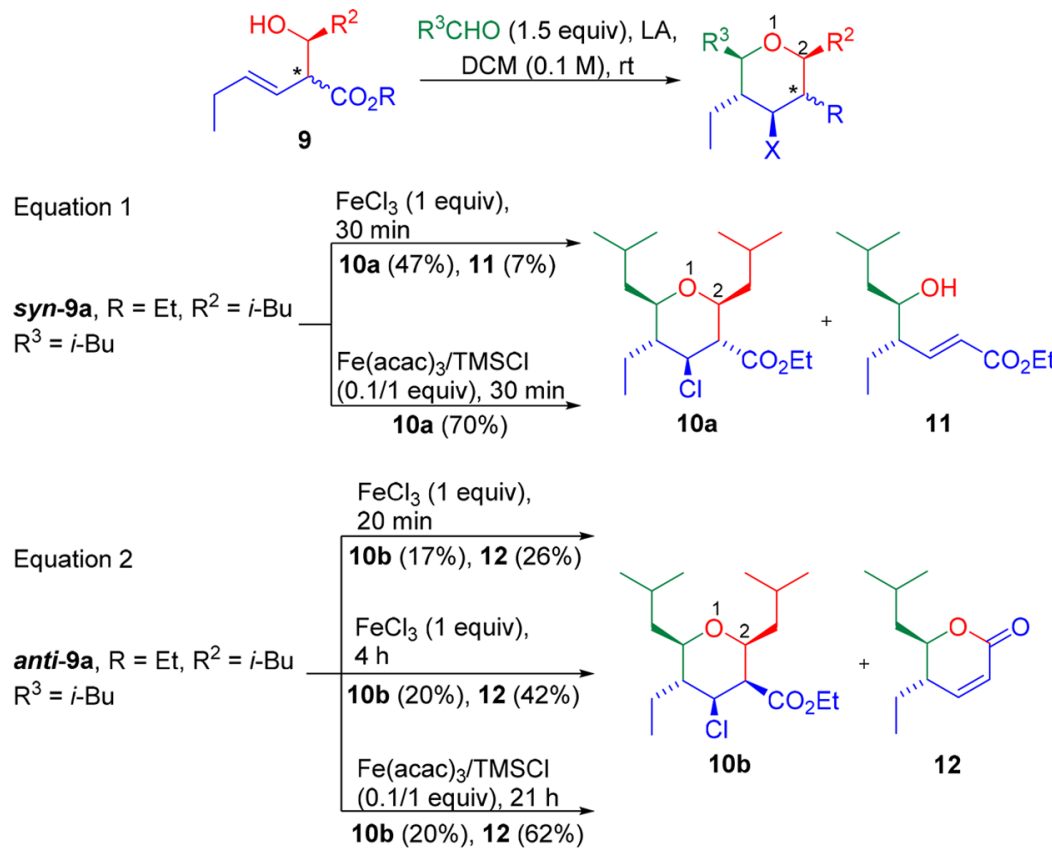

Equation 3
\[ \mathrm{Fe}(\text { acac })_{3} / \mathrm{TMSCl} \]

syn-9b, $\mathrm{R}=\mathrm{Me}, \mathrm{R}^{2}=\mathrm{Me} \stackrel{(0.1 / 1 \text { equiv }), 30 \mathrm{~min}}{\longrightarrow}$

$\mathrm{R}^{3}=\mathrm{Bu}$

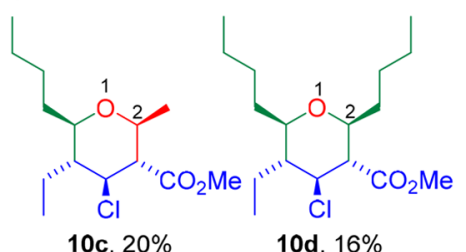

Equation 4

syn-9b, $\mathrm{R}=\mathrm{Me}, \mathrm{R}^{2}=\mathrm{Me}$

$\mathrm{R}^{3}=\mathrm{Me}$
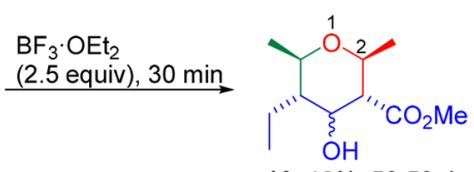

$13,12 \%, 50: 50 \mathrm{dr}$

significantly lower than the yield of $78 \%$ obtained during the synthesis of $\mathbf{5 b}\left(\mathrm{R}^{1}=\mathrm{Et}\right.$; entry 1$)$. Moreover, 2-oxonia-Cope byproduct $6 \mathrm{c}\left(\mathrm{R}^{1}=\mathrm{H}\right.$, and $\left.\mathrm{R}^{3}=\mathrm{Me}\right)$ was also isolated in $40 \%$ yield, the highest of all the examples shown until now. ${ }^{62}$ This result will be addressed again at the end of the mechanistic section (Scheme 7). Similar results were found when aldol $\mathbf{2 f}$ was combined with aromatic aldehydes to yield bicycles $5 \mathbf{x}$ and $\mathbf{5 y}$, and in these cases, traces of bicycle 5w (entries 22 and 23) were also isolated. In spite of the low yield, it should be remarked that 2,3,4,6-tetrasubstituted THPs $\mathbf{5 w} \mathbf{- 5 y}$ bear their substituents in equatorial positions; hence, they share the same core shown by natural products such as polycavernosides and clavosolides (Figure 1), which enhance the synthetic utility of the EAP protocol.

In summary, this EAP protocol allows the two-step conversion of $\mathrm{N}$-acyl oxazolidin-2-ones 3 into bicycles 5 via the formation of aldols 2. During the first step, the Evans aldol methodology permits the generation of a $\sigma \mathrm{C}-\mathrm{C}$ bond and two stereocenters in a diastereoselective fashion. The Prins cyclization of those aldols implies the creation of four $\sigma$ bonds (three $\mathrm{C}-\mathrm{O}$ and one $\mathrm{C}-\mathrm{C}$ ) and the insertion of three stereocenters (in Scheme 4, new bonds are highlighted in bold and the stereocenters with asterisks). Therefore, starting from a molecule with no chiral centers such as $3 a$, one may straightforwardly generate a high degree of structural complex- ity: bicycle $\mathbf{5 b}$ was obtained in $59 \%$ yield as a single diastereoisomer after just two steps. Nevertheless, we wondered if an even simpler alternative could be performed, by combining both Evans aldol and Prins cyclization in a onepot process. Thus, $\mathrm{N}$-acyl oxazolidin-2-one $\mathbf{3 a}$ and $\mathrm{MeCHO}$ were submitted to the Evans protocol to yield $\mathbf{2} \mathbf{b}$; once TLC analysis showed that the reaction was complete, another portion of $\mathrm{MeCHO}$ and 2.5 equiv of $\mathrm{BF}_{3} \cdot \mathrm{OEt}_{2}$ were added to the reaction medium (Scheme 4 ). To our delight, bicycle $\mathbf{5 b}$ was obtained in $54 \%$ yield, comparable to that of the two-step process. It should be emphasized that this is a truly excellent yield for a method in which five contiguous stereocenters are diastereoselectively installed and five $\sigma$ bonds are generated, meaning an average yield for each bond of $88 \%$. Additionally, this simplified protocol avoids the workup and purification of aldol $\mathbf{2} \mathbf{b}$, with the consequent saving of organic solvents and time.

The efficacy of the one-pot EAP protocol in the synthesis of $5 \mathbf{b}$ encouraged us to expand the scope by combining several $\mathrm{N}$ acyl oxazolidin-2-ones 3 and diverse aldehydes (Table 5). Entry 1 shows the result previously shown in Scheme 4. N-Acyl oxazolidin-2-one $3 \mathrm{a}$ also yielded bicycles 5 bearing different aliphatic and aromatic groups at $\mathrm{C}_{2}$ and $\mathrm{C}_{6}$ (entries 2-5). Entry 6 shows that this one-pot EAP protocol was perfectly compatible with a multigram synthesis, allowing the prepara- 


\section{Scheme 6. Derivatization of Bicycles 5}

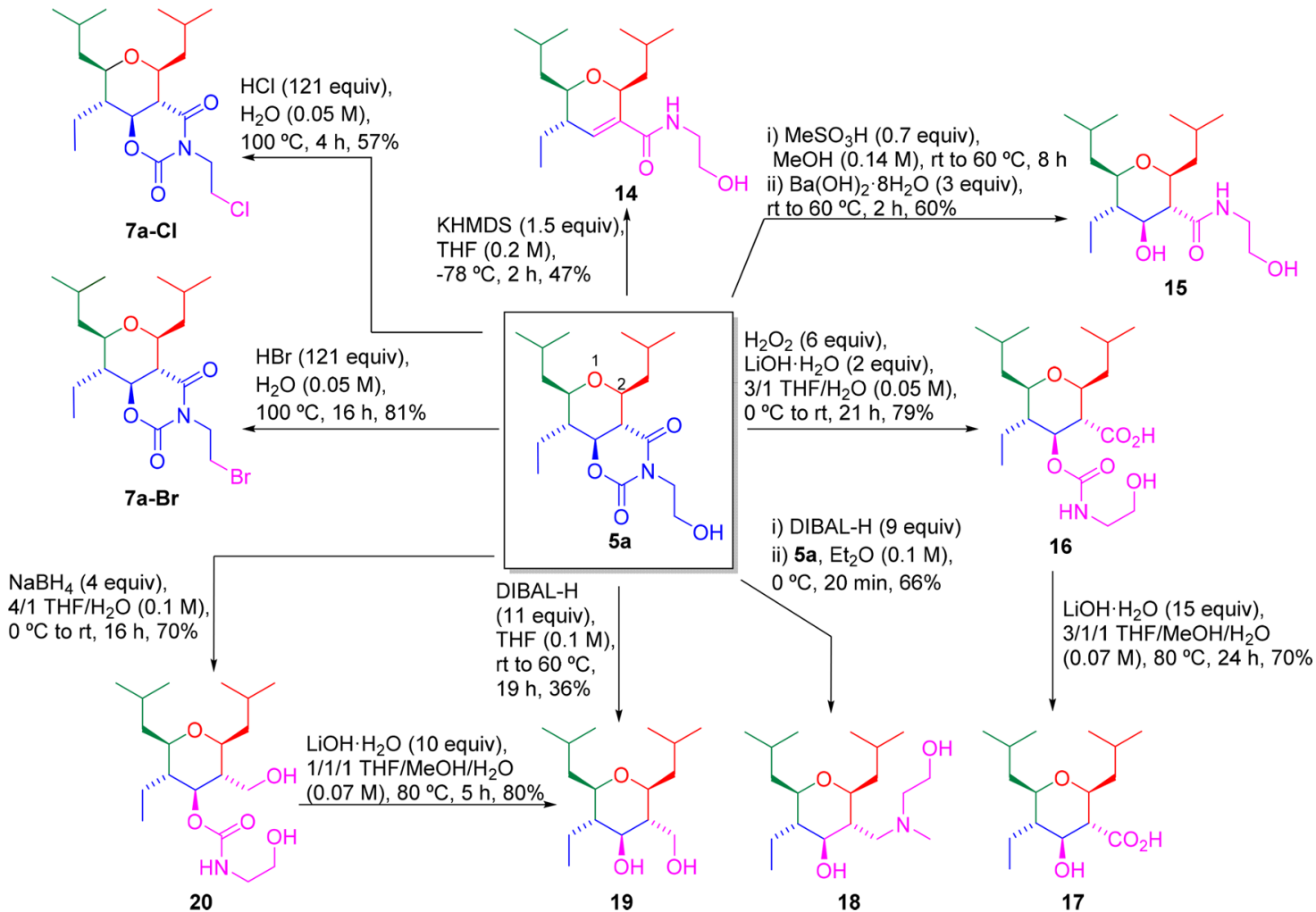

tion of $5.3 \mathrm{~g}$ of bicycle 5a from 3a with no loss of yield and enhancing the synthetic utility of this methodology. The onepot EAP protocol was also efficient starting from $N$-acyl oxazolidin-2-ones bearing an aromatic group (entry 7) and a longer linear aliphatic chain (entries 8 and 9). When a benzyl ether was present at starting material $\mathbf{3 f}$, the corresponding bicycle was achieved in $54 \%$ overall yield, as a $1.3: 1$ benzylated/nonbenzylated product mixture (entry 10).

In summary, in the two-step and one-pot versions, the EAP protocol becomes a powerful tool for the synthesis of valuable 2,3,4,5,6-pentasubstituted THPs with the general structure of 5. However, in spite of its contrasting efficacy, we still kept in mind our original aim of accessing densely substituted 4-haloTHPs 1 (Scheme 1). During the related studies described above through an understanding of the EAP cyclization, the isolated synthesis of such 4-halo-THPs (Table 2, entries 1416) really grabbed our attention, because those were the only examples in which the oxazolidin-2-one moiety acted as a mere spectator instead of undergoing a rearrangement to form the bicyclic structure. For this reason, we were interested in studying the influence of the nature of the substituent directly attached to the carbonyl group in the aldol employed as the starting material in the Prins cyclization (Scheme 5).

Thus, we devised a variation of the aldol-Prins cyclization with aldols $9 a$ and $9 \mathbf{b}$, whose structures are identical to those of Evans aldols $\mathbf{2 a}$ and $\mathbf{2} \mathbf{b}$, respectively, except for the substitution of the oxazolidin-2-one moiety for an ester group. syn-Aldol 9a was combined with $i$-BuCHO in a Prins cyclization promoted by $\mathrm{FeCl}_{3}$ to produce 4-chloro-THP 10a in $47 \%$ yield (eq 1 , top). The isolation of a small amount of homoallylic alcohol 11 (7\%) demonstrated that the Prins cyclization was competing with the 2-oxonia-Cope rearrangement. Fortunately, the generation of this undesired byproduct was suppressed when the promoter system was replaced by the
$\mathrm{Fe}(\mathrm{acac})_{3} / \mathrm{TMSCl}$ system, allowing the synthesis of $10 \mathrm{a}$ in $70 \%$ yield (eq 1, bottom). anti-Aldol 9a was also submitted to this Fe-based Prins cyclization to evaluate the influence of the stereochemistry present in the starting material (eq 2). The reaction was first stopped at $20 \mathrm{~min}$ and the nonpolar product analyzed by NMR, revealing the expected 4-chloro-THP 10b $(17 \%)$, the $\mathrm{C}_{3}$ epimer of $10 \mathrm{a}$, whose stereochemistry was unequivocally assigned on the basis of the GOESY analysis and the $J$ coupling values (eq 2, top). When the presumed remaining starting material was checked by NMR, we surprisingly discovered that it was actually a mixture of unreacted anti-aldol 9a (44\%) and $\delta$-lactone 12 (26\%). When the reaction time was increased to $4 \mathrm{~h}$, a similar yield of THP 10b was obtained (20\%), although a higher proportion of lactone 12 (42\%) with respect to the unreacted starting aldol (10\%) was detected (eq 2, middle). Eventually, when the $\mathrm{Fe}(\mathrm{acac})_{3} / \mathrm{TMSCl}$ system was employed as the promoter and the reaction time was set at $21 \mathrm{~h}$, the starting material was completely consumed and lactone $\mathbf{1 2}$ was isolated in $62 \%$ yield, although the yield of THP 10b did not improve (eq 2, bottom). ${ }^{63}$ Next, we decided to evaluate the efficacy of Febased Prins cyclization for synthesizing THPs with different chains at positions 2 and 6 . Thus, syn-aldol $9 \mathrm{~b}$ was combined with $\mathrm{BuCHO}$ and treated with the $\mathrm{Fe}(\mathrm{acac})_{3} / \mathrm{TMSCl}$ system (eq 3). Unfortunately, expected THP 10c was obtained with a poor $20 \%$ yield together with a $16 \%$ yield of THP 10d, in which a side chain exchange occurred as a consequence of the 2-oxonia-Cope rearrangement. syn-Aldol $9 \mathbf{b}$ was also submitted to the Prins cyclization mediated by 2.5 equiv of $\mathrm{BF}_{3} \cdot \mathrm{OEt}_{2}$ in pursuit of the synthesis of 4-hydroxy-THP 13 (eq 4). In contrast to the efficient synthesis of $\mathbf{5 b}$ achieved when the analogous syn-aldol $\mathbf{2} \mathbf{b}$ was treated under the same conditions [78\% and >95:5 dr (see Table 4, entry 1)], herein desired THP 13 was obtained in $12 \%$ yield and as a $1: 1$ epimeric 
Scheme 7. Computed Reaction Profile for the Cyclization of Oxonium E-INT1 and Oxonium Z-INT1

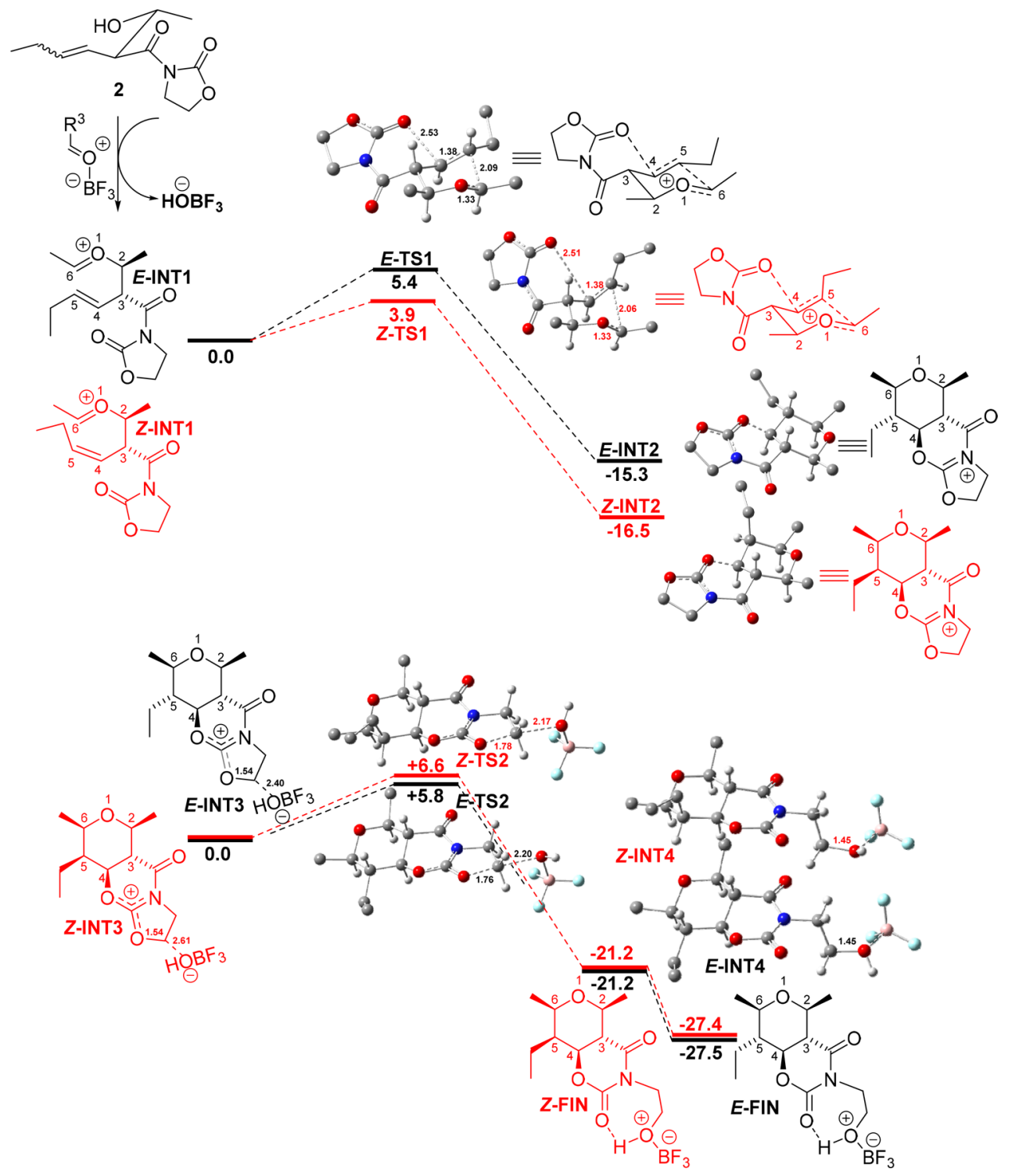

mixture at $\mathrm{C}_{4}$. Thus, according to these results, the oxazolidin2-one moiety directly attached to the carbonyl group of the aldol seems to be crucial, not only to guarantee the prevalence of the Prins cyclization product facing the 2-oxonia-Cope byproducts but also to achieve the THPs with good yields and diastereoselectivities.

Prins cyclizations illustrated in Scheme 5 constitute an unsuccessful pathway to the desired 2,3,4,5,6-pentasubstituted THPs differently functionalized at $\mathrm{C}_{3}$ and $\mathrm{C}_{4}$, which are interesting intermediates for the synthesis of THP-containing natural products. However, the EAP protocol provides efficient and robust access to this kind of THPs, although they are fused to a 1,3-oxazinane-2,4-dione ring (5). Thus, the removal of this second heterocycle should be an alternative way to access highly substituted nonbicyclic THPs, such as those commonly found in natural products (Figure 1). Bicycle 5a was chosen as the starting material, and the cleavage of the nitrogenated heterocycle was tackled through different transformations (Scheme 6). First, 5a was refluxed with an aqueous $\mathrm{HCl}$ solution for $4 \mathrm{~h}$; under these conditions, the bicyclic structure remained stable, although a chlorine atom replaced the terminal hydroxy group, yielding halo-bicycle $7 \mathrm{a}-\mathrm{Cl}$ in $57 \%$ yield. Homologous product $7 \mathbf{b}-\mathbf{C l}$ had been previously obtained with a similar yield when aldol $2 \mathrm{a}$ was submitted to the Prins cyclization mediated by the $\mathrm{BF}_{3} \cdot \mathrm{OEt}_{2} / \mathrm{TMSCl}$ system [67\% (see Table 2, entry 12)]. Similarly, when 5a was refluxed with a $\mathrm{HBr}$ aqueous solution, halo-bicycle $7 \mathrm{a}-\mathrm{Br}$ was obtained in $81 \%$ yield. Bicycle $\mathbf{5 a}$ was also submitted to an elimination reaction by being treated with KHMDS to obtain dihydropyran 14 with an amide at $\mathrm{C}_{3}{ }^{64}$ That elimination reaction allowed the release of a $\mathrm{CO}_{2}$ molecule to permit the cleavage of the bicyclic structure. Another protocol for transforming bicycle 5a into an amide was tested, affording simple access to $\beta$-hydroxy amide 15. ${ }^{65}$ Afterward, a basic hydrolysis protocol was employed in an attempt to produce a THP embedded in a $\beta$-hydroxy acid. ${ }^{66}$ Thus, treatment of 5 a with freshly prepared lithium hydroperoxide yielded THP 16 with a carboxylic acid at $C_{3}$ and a carbamate at $C_{4}$, which was successfully hydrolyzed at reflux with $\mathrm{LiOH}$ to allow access to $\beta$-hydroxy acid 17 . Eventually, several reductive protocols with DIBAL-H were analyzed, revealing noteworthy differences according to the nature of the reagent and the order of addition. When bicycle 
Scheme 8. Prins Cyclization of Enantiomeric Aldols ${ }^{a}$<smiles>CC/C=C/[C@H](C(=O)N1C(=O)CC[C@H]1Cc1ccccc1)[C@@H](C)O</smiles>

Equation 2<smiles>CC/C=C/[C@H](C(=O)N1C(=O)OCC1C(C)C)[C@@H](C)O</smiles>

Equation 3

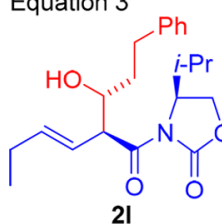

Equation 4<smiles>CC/C=C/[C@H](C(=O)N1C(=O)O[C@H](c2ccccc2)[C@H]1C)C(O)Br</smiles><smiles>CC[C@H]1[C@@H](C)O[C@@H](C)[C@H]2C(=O)N([C@H](CO)CP)C(=O)O[C@H]12</smiles>

5ag (62\%)<smiles>CC[C@H]1C(C)OC(C)[C@H](C(=O)N2C(=O)OC[C@H]2Cc2ccccc2)[C@H]1O</smiles>

21a (9\%)

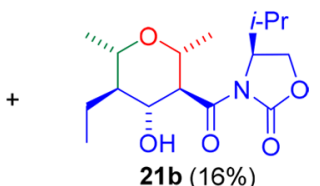

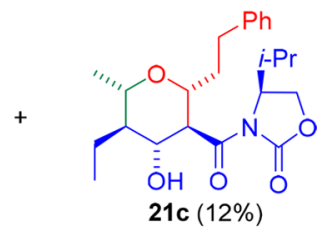

${ }^{a}$ Reaction conditions: MeCHO (1.5 equiv), Lewis acid, DCM (0.1 M), rt, $30 \mathrm{~min}$. All products were obtained with a >95:5 dr except 5ah (92:8 dr).

5a was added over an ice-cooled DIBAL-H solution, 4hydroxy-THP 18, bearing a tertiary amine, was obtained because of the total reduction of both carbonyl groups. However, when DIBAL-H was added in a dropwise fashion to a solution of bicycle $5 \mathrm{a}$ in THF and then refluxed, diol 19 was obtained. ${ }^{67}$ The use of $\mathrm{NaBH}_{4}$ as the source of hydride led to carbamate $20{ }^{68}$ hydrolysis of 20 yielded diol $19 .^{66}$ The conclusion deduced from this derivatization screening is that bicycles 5, easily obtained via our EAP protocol, constitute a versatile platform for accessing a substantial family of highly substituted THPs bearing various functional groups.

A mechanistic model for the Prins cyclization using $E$ - and Z-homoallylic alcohols 2 obtained from the Evans aldol addition is outlined below. Considering that the variation of the reaction temperature almost did not affect the diastereoselectivity of the reaction and modified only the reaction time, ${ }^{51}$ a kinetically controlled mechanism would be expected. In addition, from the experimental results, it seems that the oxazolidin-2-one group is not a mere spectator in the process, because the reaction fails when it is replaced by an ester group (Scheme 5). We performed DFT calculations to delve into the complete diastereoselectivity of the Lewis acid-catalyzed Prins cyclization described above. In this regard, we computed the reaction profile $\left[\mathrm{SCRF}\left(\mathrm{CH}_{2} \mathrm{Cl}_{2}\right)-\mathrm{B} 3 \mathrm{LTP} / 6-3 \lg (\mathrm{d})\right.$ level] involving the oxocarbenium that resulted from the condensation of simple allylic alcohol $2 \mathbf{b}\left(\mathrm{R}^{1}=\mathrm{Et}\right.$, and $\left.\mathrm{R}^{2}=\mathrm{Me}\right)$ for both $E$ - and $Z$-isomers (E-INT1 and Z-INT1, respectively) in the presence of $\mathrm{BF}_{3}$ as the Lewis acid (Scheme 7). We speculate that trifluorohydroxyborate is formed from the $\mathrm{BF}_{3}$ used and will be important during the overall mechanism leading to the final tetrahydropyran. Relative enthalpies $\Delta G$ $(298 \mathrm{~K})$ and bond distances are given in kilocalories per mole and angstroms, respectively. The numbering of the figures is arbitrary and used for discussion. Only representative hydrogens are shown. DFT calculations, in the gas phase, were performed at the $\mathrm{B} 3 \mathrm{LYP} / 6-31 \mathrm{G}(\mathrm{d})$ level and punctually corrected to include solvation in DCM, using the SCRF method, used by default in Gaussian. The transition states were confirmed with the corresponding force calculations, ensuring the presence of a single imaginary frequency in all cases. For the determination of the E-INT3 and Z-INT3 complexes, the basis set superposition error was taken into account using the "counterpoise" method.

We first discuss the cyclization for the E-isomer of the double bond at the homoallylic alcohol. As depicted in Scheme 7, oxonium ion E-INT1 evolves exothermically $\left(\Delta E_{\mathrm{r}}=-15.3\right.$ $\mathrm{kcal} / \mathrm{mol}$ ) to carbocation E-INT2 through double-bond nucleophilic attack. Transition state E-TS1 $\left(\Delta E_{\mathrm{r}}=+5.4\right.$ $\mathrm{kcal} / \mathrm{mol}$ ) adopts a chairlike conformation caused by the arrangement of the $\mathrm{N}$-acyl oxazolidin-2-one on the substrate. In this transition state, all substituents are located in the equatorial position setting the relative configurations of $\mathrm{C}_{5}$ and $\mathrm{C}_{6}$ in the product. The obtained carbocation, via $E$-TS1, allows the carbonyl nucleophilic equatorial attack of the oxazolidin-2one ensuring the stereochemistry at $\mathrm{C}_{4}$ in E-INT2. This step is highly exothermic $\left(\Delta E_{\mathrm{r}}=-15.3 \mathrm{kcal} / \mathrm{mol}\right)$ as a result of the stabilization of the positive charge by the adjacent heteroatoms. The existence of a true bond between the oxazolidin-2-one carbonyl oxygen and the electron deficit center (numbered as 4 in the scheme), shown with a dotted 
line, was confirmed by the AIM (atom in molecules) methodology $[6-311+g(d, p) / / B 3 L Y P / 6-31 G(d)]$, which justifies the stereochemistry of this center. In a similar manner, the double-bond nucleophilic attack on the electrophilic position in Z-INT1 generates cyclic tetrahydropyran Z-INT2 in an exothermic process $\left(\Delta E_{\mathrm{r}}=-16.5 \mathrm{kcal} / \mathrm{mol}\right)$. Transition state Z-TS1 $\left(\Delta E_{\mathrm{r}}=+3.9 \mathrm{kcal} / \mathrm{mol}\right)$ adopts again a chairlike conformation, placing the ethyl group in a pseudoaxial position. The obvious consequence of this location is the resulting geometry at the $\mathrm{C}_{5}$ position yielding diastereoisomer Z-INT2, as it was observed experimentally (Scheme 3, eq 3). The reaction ends with an $S_{N} 2$ nucleophilic attack of the trifluorohydroxyborate (formed during the condensation reaction) generating the primary alcohol and the corresponding 1,3-oxazinane-2,4-dione as the leaving group. We first performed calculations over van der Walls complex E-INT3 having as its origin the $E$-isomer of the double bond of the homoallylic alcohol. This process is highly exothermic yielding E-INT4 $\left(\Delta E_{\mathrm{r}}=-21.2 \mathrm{kcal} / \mathrm{mol}\right)$ through a low computed activation barrier for E-TS2 $\left(\Delta E_{\mathrm{r}}=+5.8 \mathrm{kcal} / \mathrm{mol}\right)$. Lineal intermediate $\boldsymbol{E}$-INT4 evolves to the more stable and final $\mathrm{BF}_{3}$ complex E-FIN stabilized by an intramolecular $\mathrm{O} \cdots \mathrm{HO}$ hydrogen bond. An experimental confirmation of this last $\mathrm{S}_{\mathrm{N}} 2$ nucleophilic attack is provided for the formation of the corresponding acetate when acetic acid was used as a solvent, presumably via the formation of a $\mathrm{BF}_{3} \cdot \mathrm{HOAc}$ complex (Table 3, entry 6). ${ }^{69}$ As expected, calculations over Z-isomer Z-INT3 provide results almost identical to those for the $E$-isomer, providing exothermically $Z$-INT4 $\left(\Delta E_{\mathrm{r}}=-21.2 \mathrm{kcal} / \mathrm{mol}\right)$ via low-energy transition state Z-TS2 $\left(\Delta E_{\mathrm{r}}=+6.6 \mathrm{kcal} / \mathrm{mol}\right)$. In a similar manner, the reaction ends with the intramolecular formation of an H-bond in final THP complex Z-FIN. Thus, Scheme 7 justifies the formation of bicyclic compounds 5, ratifying the experimental results. However, it was surprising that when we started from aldol $2 \mathbf{f}$ bearing a terminal alkene $\left(\mathrm{R}^{1}=\mathrm{H}\right)$, the 2-oxonia-Cope products were observed at significant levels (Table 4, entries 21-23). To find a theoretical justification of this phenomenon, we proceeded to repeat the same calculations shown in Scheme 7 for $R^{1}=H$, also generating a reaction coordinate from the approach $\mathrm{C}_{5}-$ $\mathrm{C}_{6}$. We observed that in the product equivalent to E-INT1 (or Z-INT1), the hypothetical carbocation at $\mathrm{C}_{4}$ is not assisted by the carbonyl of the oxazolidin-2-one. In theses cases, the approach leads directly to rearranged product $6\left(\Delta E_{\mathrm{r}}=-9.0\right.$ $\mathrm{kcal} / \mathrm{mol}$, and $\left.\Delta E_{\mathrm{r}}{ }^{*}=3.0 \mathrm{kcal} / \mathrm{mol}\right)$. Clearly, the substitution of the terminal vinyl position in aldols $2\left(\mathrm{R}^{1} \neq \mathrm{H}\right)$ stabilizes the charge at $\mathrm{C}_{4}$ to induce the approximation of the oxazolidin2-one ring, favoring the formation of tricyclic intermediate $\boldsymbol{E}$ INT2 or Z-INT2 (Scheme 7). Otherwise, the $[3,3]$ sigmatropic rearrangement is observed.

To extend the applicability of the EAP cyclization, the enantiomeric version was tested employing chiral alcohols $2 \mathbf{i}-$ m previously obtained (Table 1 , entries $18-22$, respectively). ${ }^{70}$ Thus, Prins cyclization of acetaldehyde and aldol $2 \mathbf{i}$, bearing a benzyl group in the oxazolidin-2-one, allowed us to obtain expected bicycle 5 ag in $62 \%$ yield but also led to the unprecedented isolation of THP 21a in 9\% yield (Scheme 8, eq 1). ${ }^{71}$ THPs as 21 (termed THP-Xc to highlight the presence of the nonrearranged oxazolidin-2-one in their structures) were not detected in any of the Prins cyclizations previously studied; therefore, we reasoned that the chiral nature of the oxazolidin-2-one motif was involved in their generation. In an attempt to control the relative amount of isomers $\mathbf{5}$ and $\mathbf{2 1}$, a screening of Lewis acids was unsuccessfully performed. ${ }^{72}$ However, it must be pointed out that, from a synthetic point of view, the presence of this pair of products is not a handicap because under hydrolysis or reduction conditions both must evolve to the same 2,3,4,5,6-pentasubstituted THP (see products 17 and 19 in Scheme 6). When the Prins cyclization was performed by employing acetaldehyde and aldol $\mathbf{2 k}$, in which the oxazolidin-2-one presents an $i$-Pr group instead of a benzyl group, both bicyclic product 5ah (43\%) and THP-Xc 21 b (16\%) were obtained again, as reflected in eq 2 . In a similar manner, cyclization using aromatic alcohol $\mathbf{2 l}$ provided a mixture of bicycle 5 ai and THP-Xc 21c in 57\% overall yield (eq 3). Finally, we decided to study the Prins cyclization between $n$-pentanal and aldol $2 \mathrm{~m}$, in which the oxazolidin-2-one motif presents substituents at both positions adjacent to $\mathrm{N}$ and $\mathrm{O}$ (eq 4). THP-Xc 21d was obtained with an apparently disappointing $10 \%$ yield, though this result is really meaningful from a mechanistic point of view. As shown in Scheme 7, the nucleophilic attack of the trifluorohydroxyborate on the position adjacent to the $\mathrm{O}$ of the oxazolidin-2-one usually leads to the generation of bicycles 5 . Nevertheless, in this case, the presence of the phenyl substituent in that position prevents the nucleophilic attack over the oxazolidin-2-one, yielding exclusively a THP-Xc 21.

Eventually, many of all these new products were biologically evaluated. Our interest in the development of biostudies concerning THPs arises from the high incidence of this structural motif in bioactive natural products (Figure 1) and from their inherent bioactivity. As antimicrobial and antifungal activities are recurrently associated with $\mathrm{THPs}^{4,5,8,9,13}$ we decided to evaluate the antimicrobial activity of 33 of the compounds obtained in the current study ${ }^{73}$ against Grampositive and Gram-negative bacteria and the yeast $C$. albicans. The $\mathrm{MIC}_{50}$ values listed in Table 6 clearly show that the effect

Table 6. Antimicrobial Activity $\left(\mathrm{MIC}_{50}, \mu \mathrm{g} / \mathrm{mL}\right)$ of Selected Compounds against the Susceptible Gram-Positive Bacteria $^{a}$

$\begin{array}{cccc}\text { compound } & \begin{array}{c}\text { Staphylococcus epidermidis } \\ \text { ATCC } 14990\end{array} & \begin{array}{c}\text { S. aureus MRSA } \\ \text { ULL1 }\end{array} & \begin{array}{c}\text { B. subtilis } \\ \text { ATCC 6051 }\end{array} \\ \text { 5c } & 28 & 28 & 25 \\ \text { 5n } & >40 & >40 & 11 \\ \mathbf{5 z} & >40 & >40 & 40 \\ \mathbf{5 a d} & 28 & 28 & 18 \\ 7 \mathbf{7}-\mathbf{C l} & >40 & >40 & 3\end{array}$

${ }^{a}$ All assays were performed in triplicate. All the compounds assayed were inactive $\left(\mathrm{MIC}_{50}>40 \mu \mathrm{g} / \mathrm{mL}\right.$ ) against Gram-positive (Bacillus cereus and S. aureus) and Gram-negative (E. coli, Pseudomonas aeruginosa, and Proteus mirabilis) bacteria and the yeast $C$. albicans CECT 1039.

of the compounds is limited to Gram-positive bacteria. B. subtilis was more sensitive than the genus Staphylococcus, although compounds $\mathbf{5 c}$ and $\mathbf{5 a d}$ displayed activity against Staphylococcus aureus methicillin resistance $\left(\mathrm{MIC}_{50}=28 \mu \mathrm{g} /\right.$ $\mathrm{mL})$. Structural analyses of the compounds suggest that the growth inhibitory capacities of such products are strictly linked to the presence of the bicyclic structure and to a functionalization at positions 2 and 6 different from the methyl group. The presence of $i$-Bu groups in these positions and a chlorine atom replacing the terminal hydroxy group, as observed in compound $\mathbf{7 a}-\mathbf{C l}$, increased the activity $\left(\mathrm{MIC}_{50}=\right.$ 
$3 \mu \mathrm{g} / \mathrm{mL})$. Furthermore, the presence of a butyl group at position 2 and 6 (5c and 5ad, respectively) broadens the activity to the genus Staphylococcus.

\section{CONCLUSIONS}

The EAP protocol has emerged as an efficient tool for the transformation of $\beta, \gamma$-unsaturated $N$-acyl oxazolidin-2-ones into 2,3,4,5,6-pentasubstituted THPs. These oxacycles were obtained in an unprecedented bicyclic form because of the rearrangement suffered in the reaction medium by the auxiliary borne by the starting material. Two variants of the EAP protocol have been developed, a two-step sequence and a simpler one-pot variant, both exhibiting high tolerance to various functional groups and allowing the introduction of aromatic and aliphatic moieties at positions 2, 5, and 6 of the THPs. The one-pot version permitted the introduction of five adjacent stereocenters with diastereoisomeric ratios generally greater than 95:5, as well as the generation of three $\mathrm{C}-\mathrm{O}$ and two $\mathrm{C}-\mathrm{C}$ bonds with average yields of $\leq 90 \%$. The two-step strategy allowed the production of both racemic and chiral THPs, and the modulation of the stereochemistry of the starting unsaturated aldol allowed the fine-tuning of the stereochemical pattern shown in the final THP, enabling thus access to different cores of several natural products. Computational studies were consistent with those stereochemical essays and with the observed rearrangement of the oxazolidin-2-one motif. It was also revealed that the presence of the oxazolidin2-one ring in the starting materials was absolutely necessary to guarantee, on one hand, the diastereoselectivity of the process and to deactivate, on the other, the competing 2-oxonia-Cope rearrangement usually concomitant with Prins cyclization. The meticulous screening of the reaction conditions led us to establish as optimal the employment of DCM as the solvent and 2.5 equiv of $\mathrm{BF}_{3} \cdot \mathrm{OEt}_{2}$ as the promoter, although its combination with $\mathrm{TMSCl}$ permitted the direct synthesis of chlorinated derivatives. Other Lewis acids such as $\mathrm{FeBr}_{3}$, $\mathrm{TMSBr}$, and TMSI were also able to yield halogenated bicycles or even 4-halo-2,3,4,5,6-pentasubstituted THPs, but these reaction conditions have not yet been optimized. Direct halogenation of the bicyclic THPs was also achieved, and it was found that these compounds constitute a versatile platform for accessing a considerable diversity of simpler nonbicyclic THPs bearing amines, amides, carbamates, carboxylic acids, and hydroxy groups. Bioassays showed that some of the synthesized THPs were active against Gram-positive bacteria, yielding the best values of the $\mathrm{MIC}_{50}$ for $B$. subtilis. We expect that this complete study detailed herein will lay the foundation for expanding the range of synthetic applications of the EAP protocol.

\section{EXPERIMENTAL SECTION}

General Experimental Methods. Atoms of all the compounds were numbered according to the IUPAC name. All reagents were commercially available and used as received without further purification, unless noted otherwise. A 3.3 M solution of acetaldehyde in DCM was prepared by diluting $23 \mathrm{~mL}$ of commercial and volatile acetaldehyde in $100 \mathrm{~mL}$ of dry DCM. The molarity of the solution was checked by ${ }^{1} \mathrm{H}$ NMR spectroscopy. The solution was stored at 2$8{ }^{\circ} \mathrm{C}$ under Ar, being stable for at least 12 months. $\mathrm{BF}_{3} \cdot \mathrm{OEt}_{2}$ (bp 129 ${ }^{\circ} \mathrm{C}$ ) was distilled and stored at $-18{ }^{\circ} \mathrm{C}$ under Ar. All solvents were dried and distilled under $\mathrm{Ar}$ immediately prior to use or stored appropriately. THF was refluxed over sodium and benzophenone. DCM was distilled from $\mathrm{CaH}_{2}$. Reactions were monitored by thinlayer chromatography (TLC) analysis employing UV light (365 nm), a phosphomolybdic acid solution $10 \mathrm{wt} \%$ in methanol, or a vanillin solution ( $6 \mathrm{~g}$ of vanillin, $450 \mathrm{~mL}$ of ethanol, $40 \mathrm{~mL}$ of $\mathrm{AcOH}$, and 30 $\mathrm{mL}$ of $\left.\mathrm{H}_{2} \mathrm{SO}_{4}\right)$. TLC was run on silica gel $60 \mathrm{~F}_{254}$ aluminum sheets. Flash chromatography was performed with silica gel (230-400 mesh) as the stationary phase and mixtures of $n$-hexane and EtOAc, in different proportions given in each case, as the mobile phase. Melting points were determined on a Büchi B-540 model. Optical rotations were determined on a PerkinElmer 343 polarimeter using a sodium lamp operating at $589 \mathrm{~nm} .{ }^{1} \mathrm{H} \mathrm{NMR}(400,500$, or $600 \mathrm{MHz})$ and ${ }^{13} \mathrm{C}$ NMR $(100,125$, or $150 \mathrm{MHz})$ spectra were recorded at room temperature; chemical shifts $(\delta)$ are reported in parts per million, and coupling constants $(J)$ are given in hertz. ${ }^{1} \mathrm{H}$ NMR spectra are referenced to the resonance from residual $\mathrm{CHCl}_{3}$ at $7.26 \mathrm{ppm}$; multiplicity is expressed by the abbreviations $\mathrm{m}$ (multiplet), br (broad signal), s (singlet), d (doublet), $\mathrm{t}$ (triplet), $\mathrm{q}$ (quartet), and combinations thereof for more highly coupled systems. ${ }^{13} \mathrm{C}$ NMR spectra are referenced to the central peak of the signal from $\mathrm{CDCl}_{3}$ at $77.16 \mathrm{ppm}$; multiplicity was assigned from DEPT135 and DEPT90 experiments and is expressed by the abbreviations $\mathrm{s}(\mathrm{C}), \mathrm{d}(\mathrm{CH}), \mathrm{t}$ $\left(\mathrm{CH}_{2}\right)$, and $\mathrm{q}\left(\mathrm{CH}_{3}\right)$. Structures were elucidated according to literature precedents or using two-dimensional NMR techniques such as COSY, HSQC, edited HSQC, and/or HMBC; spatial elucidation was performed via NMR according to the GOESY technique. Mass spectra were recorded by using electronic impact (EI-TOF $70 \mathrm{eV}$ ) or by using electrospray ionization (ESI $\left.{ }^{+}-\mathrm{TOF}\right)$, as specified in each case.

Antimicrobial Assay. The strains used to determine antimicrobial activity included S. aureus ATCC 6538, S. aureus methicillin-resistant (MRSA ULL1, clinical isolate, University of La Laguna), S. epidermidis ATCC 14990, B. subtillis ATCC 6051, B. cereus ATCC 21772, E. coli ATCC 9637, P. mirabilis CECT 170 (from the Colección Española de Cultivos Tipo), P. aeruginosa AK958 (from the Department of Microbiology collection from the University of British Columbia, Vancouver, BC), and C. albicans CECT1032. The $\mathrm{MIC}_{50}$ was determined for each compound in triplicate, by the microdilution method (range of $0.08-40 \mu \mathrm{g} / \mathrm{mL}$ ) in 96-well microtiter plates. ${ }^{74}$ Wells with the same proportion of DMSO were used as controls, and the level never exceeded $1 \%(\mathrm{v} / \mathrm{v})$. The starting microorganism density was approximately $1 \times 10^{5}$ to $5 \times 10^{5}$ colony forming units (CFU) per milliliter, and growth was monitored by measuring the increase in the optical density at $550 \mathrm{~nm}$ with a microplate reader (Tecan Group Ltd., Mannedorf, Switzerland). All wells with no visible growth were subcultured by transferring them in duplicate $(100 \mu \mathrm{L})$ to agar plates. After overnight incubation, colony counts were performed and the $\mathrm{MIC}_{50}$ was defined as the lowest concentration of compound affecting a 50\% decrease in growth at the end of the incubation period relative to untreated controls.

General Procedure for the Synthesis of the $\beta, \gamma$-Unsaturated Carboxylic Acids 4. A mixture of the aldehyde, malonic acid (1.1 equiv), and NMM (1.1 equiv), prepared under Ar, was heated at 95 ${ }^{\circ} \mathrm{C}$ until the reaction was complete ( $2-8 \mathrm{~h}$ approximately). After that, the mixture was cooled to $0{ }^{\circ} \mathrm{C}$, treated with a $2 \mathrm{M}$ aqueous solution of $\mathrm{H}_{2} \mathrm{SO}_{4}$ (1.1 equiv), and extracted three times with DCM. The combined organic layers were washed with water, dried over anhydrous $\mathrm{MgSO}_{4}$, filtered, concentrated, and purified by flash chromatography to yield acids $4 .^{75}$ Acids $4 a-4 c$ are commercially available and were used as received without further purification. Acids $\mathbf{4 d - 4 f}$ were described in our previous publication ${ }^{30}$ and were stable for at least 12 months stored under $\mathrm{Ar}$ at $-18{ }^{\circ} \mathrm{C}$.

General Procedure for the Synthesis of the $\mathrm{N}$-Acyl Oxazolidin-2-ones 3. All the subsequent operations were performed under an $\mathrm{Ar}$ atmosphere. To a solution of the carboxylic acid in dry THF $(0.16 \mathrm{M})$ was added, at $0{ }^{\circ} \mathrm{C}$, TEA ( 1.1 equiv). After $5 \mathrm{~min}$, pivaloyl chloride (1.3 equiv) was also added at $0{ }^{\circ} \mathrm{C}$, producing a suspension of the mixed acid anhydride that was stirred for $1 \mathrm{~h}$ at $\mathrm{rt}$. Meanwhile, in another flask, a solution of the oxazolidin-2-one (1.3 equiv) in dry THF $(0.3 \mathrm{M})$ was cooled to $-78^{\circ} \mathrm{C}$, treated dropwise with a $2.5 \mathrm{M}$ solution of $n$-butyllithium in hexanes (1.2 equiv), and kept at that temperature until it was poured (a slow addition is not required) into the $-78{ }^{\circ} \mathrm{C}$ cooled suspension of the anhydride. After that, the mixture was allowed to warm to $\mathrm{rt}$, and after $15 \mathrm{~h}$, the 
reaction was stopped with a saturated $\mathrm{NH}_{4} \mathrm{Cl}$ aqueous solution. Then, it was extracted three times with EtOAc, and the combined organic layers were dried over anhydrous $\mathrm{MgSO}_{4}$, filtered, concentrated, and purified by flash chromatography to yield desired compound 3 . The $\mathrm{N}$-acyl oxazolidin-2-ones are usually slightly more apolar than the starting carboxylic acid. Compounds with the structure of 3 are stable for 6 months if they are properly stored under $\mathrm{Ar}$ at $-18^{\circ} \mathrm{C}$, although they begin to decompose thereafter. $N$-Acyl oxazolidin-2-ones $3 \mathbf{a}-\mathbf{3 f}$ were described in our previous publication. ${ }^{30}$

$(R, E)-4-B e n z y l-3-(h e x-3-e n o y l) o x a z o l i d i n-2-o n e ~(3 g)$. Acid 4a (1 $\mathrm{mL}, 8.18 \mathrm{mmol}$ ) was subjected to the general procedure for the synthesis of $\mathrm{N}$-acyl oxazolidin-2-ones 3. Purification by flash chromatography $(11 \mathrm{~cm}$ of height of silica gel, $n$-hexane/EtOAc $75: 25)$ provided the title compound together with the rest of the pivaloyl chloride. To remove that contaminant, the mixture was solved in $\mathrm{Et}_{2} \mathrm{O}(30 \mathrm{~mL})$ and washed with $\mathrm{H}_{2} \mathrm{O}(10 \times 30 \mathrm{~mL})$. The organic layer was dried over anhydrous $\mathrm{MgSO}_{4}$, filtered, and concentrated to yield product $3 \mathrm{~g}(2.00 \mathrm{~g}, 90 \%)$ as a yellowish oil: $R_{f}=0.44$ ( $n$-hexane/EtOAc 70:30), 0.85 ( $n$-hexane/EtOAc 20:80); $[\alpha]^{25}-64.7\left(c 1.0, \mathrm{CHCl}_{3}\right) ;{ }^{1} \mathrm{H}$ NMR $\left(500 \mathrm{MHz}, \mathrm{CDCl}_{3}\right) \delta 1.02(\mathrm{t}$, $\left.J=7.5 \mathrm{~Hz}, 3 \mathrm{H}, \mathrm{H}_{6^{\prime}}\right), 2.06-2.13\left(\mathrm{~m}, 2 \mathrm{H}, \mathrm{H}_{5^{\prime}}\right), 2.78(\mathrm{dd}, J=13.5,9.8$ $\left.\mathrm{Hz}, 1 \mathrm{H}, 1 \times \mathrm{PhCH}_{2} \mathrm{C}_{4}\right), 3.30(\mathrm{dd}, J=13.4,3.2 \mathrm{~Hz}, 1 \mathrm{H}, 1 \times$ $\left.\mathrm{PhCH}_{2} \mathrm{C}_{4}\right), 3.61-3.73\left(\mathrm{~m}, 2 \mathrm{H}, \mathrm{H}_{2^{\prime}}\right), 4.16-4.23\left(\mathrm{~m}, 2 \mathrm{H}, \mathrm{H}_{5}\right), 4.67$ (ddt, $\left.J=9.6,7.5,3.2 \mathrm{~Hz}, 1 \mathrm{H}, \mathrm{H}_{4}\right), 5.61(\mathrm{dtt}, J=15.6,6.6,1.3 \mathrm{~Hz}, 1 \mathrm{H}$, $\left.\mathrm{H}_{3^{\prime}}\right), 5.70\left(\mathrm{dtt}, J=15.6,6.1,1.3 \mathrm{~Hz}, 1 \mathrm{H}, \mathrm{H}_{4^{\prime}}\right), 7.19-7.22(\mathrm{~m}, 2 \mathrm{H})$, 7.27-7.30 (m, $1 \mathrm{H}), 7.31-7.35(\mathrm{~m}, 2 \mathrm{H}) ;{ }^{13} \mathrm{C}$ NMR $(125 \mathrm{MHz}$, $\left.\mathrm{CDCl}_{3}\right) \delta 13.6\left(\mathrm{q}, \mathrm{C}_{6^{\prime}}\right), 25.8\left(\mathrm{t}, \mathrm{C}_{5^{\prime}}\right), 38.0\left(\mathrm{t}, \mathrm{C}_{2^{\prime}}\right.$ or $\left.\mathrm{PhCH}_{2} \mathrm{C}_{4}\right), 39.3$ $\left(\mathrm{t}, \mathrm{C}_{2^{\prime}}\right.$ or $\left.\mathrm{PhCH}_{2} \mathrm{C}_{4}\right), 55.4\left(\mathrm{~d}, \mathrm{C}_{4}\right), 66.4\left(\mathrm{t}, \mathrm{C}_{5}\right), 120.1\left(\mathrm{~d}, \mathrm{C}_{3^{\prime}}\right), 127.5$ (d, Ph), 129.1 (d, 2C, Ph), 129.6 (d, 2C, Ph), 135.4 (s, Ph), 137.3 (d, $\left.\mathrm{C}_{4^{\prime}}\right), 153.5\left(\mathrm{~s}, \mathrm{C}_{2}\right), 172.1\left(\mathrm{~s}, \mathrm{C}_{1^{\prime}}\right) ; \mathrm{MS}(\mathrm{EI}) \mathrm{m} / z$ (relative intensity) $273(\mathrm{M})^{+}(55), 178(28), 97(\mathrm{M}-\text { oxazolidin-2-one })^{+}(55), 96$ (100); HRMS calcd for $\mathrm{C}_{16} \mathrm{H}_{19} \mathrm{NO}_{3}\left[(\mathrm{M})^{+}\right]$273.1365, found 273.1362 .

(S,E)-3-(Hex-3-enoyl)-4-isopropyloxazolidin-2-one (3h). Acid 4a $(0.5 \mathrm{~mL}, 4.09 \mathrm{mmol})$ and $(S)$-4-isopropyloxazolidin-2-one $(641 \mathrm{mg}$, $4.91 \mathrm{mmol}, 1.2$ equiv) were subjected to the general procedure for the synthesis of $N$-acyl oxazolidin-2-ones 3 and yielded, after purification by flash chromatography ( $32 \mathrm{~cm}$ of height of silica gel, $n$-hexane/ EtOAc 95:5), compound $3 \mathrm{~h}(765 \mathrm{mg}, 83 \%)$ as a thick colorless oil: $R_{f}$ $=0.32$ ( $n$-hexane $/$ EtOAc 80:20), 0.55 ( $n$-hexane $/$ EtOAc 80:20, three times); $[\alpha]^{25}{ }_{\mathrm{D}}+75.1\left(c 1.0, \mathrm{CHCl}_{3}\right) ;{ }^{1} \mathrm{H} \mathrm{NMR}\left(500 \mathrm{MHz}, \mathrm{CDCl}_{3}\right) \delta$ $0.86\left(\mathrm{dd}, J=6.8,0.9 \mathrm{~Hz}, 3 \mathrm{H},\left(\mathrm{CH}_{3}\right)_{2} \mathrm{CHC}_{4}\right), 0.90(\mathrm{dd}, J=7.0,1.2$ $\left.\mathrm{Hz}, 3 \mathrm{H}, 1 \times\left(\mathrm{C}_{3}\right)_{2} \mathrm{CHC}_{4}\right), 0.98\left(\mathrm{td}, J=7.6,1.2 \mathrm{~Hz}, 3 \mathrm{H}, \mathrm{H}_{6^{\prime}}\right), 2.02-$ $2.09\left(\mathrm{~m}, 2 \mathrm{H}, \mathrm{H}_{5^{\prime}}\right), 2.34-2.41\left(\mathrm{~m}, 1 \mathrm{H},\left(\mathrm{CH}_{3}\right)_{2} \mathrm{CHC}_{4}\right), 3.59(\mathrm{dd}, J=$ $\left.16.7,6.6 \mathrm{~Hz}, 1 \mathrm{H}, \mathrm{H}_{2^{\prime}}\right), 3.70\left(\mathrm{dd}, J=17.0,6.7 \mathrm{~Hz}, 1 \mathrm{H}, \mathrm{H}_{2^{\prime}}\right.$ ), 4.20 (ddd, $\left.J=9.1,3.0,0.9 \mathrm{~Hz}, 1 \mathrm{H}, \mathrm{H}_{5}\right), 4.25-4.28\left(\mathrm{~m}, 1 \mathrm{H}, \mathrm{H}_{5}\right), 4.40-4.44(\mathrm{~m}$, $\left.1 \mathrm{H}, \mathrm{H}_{4}\right), 5.53-5.60\left(\mathrm{~m}, 1 \mathrm{H}, \mathrm{H}_{3^{\prime}}\right), 5.63-5.70\left(\mathrm{~m}, 1 \mathrm{H}, \mathrm{H}_{4^{\prime}}\right) ;{ }^{13} \mathrm{C} \mathrm{NMR}$ $\left(125 \mathrm{MHz}, \mathrm{CDCl}_{3}\right) \delta 13.6\left(\mathrm{q}, \mathrm{C}_{6^{\prime}}\right), 14.8\left(\mathrm{q},\left(\mathrm{CH}_{3}\right)_{2} \mathrm{CHC}_{4}\right), 18.1(\mathrm{q}$, $\left.\left(\mathrm{CH}_{3}\right)_{2} \mathrm{CHC}_{4}\right), 25.7\left(\mathrm{t}, \mathrm{C}_{5^{\prime}}\right), 28.5\left(\mathrm{~d},\left(\mathrm{CH}_{3}\right)_{2} \mathrm{CHC}_{4}\right), 39.3\left(\mathrm{t}, \mathrm{C}_{2^{\prime}}\right)$, $58.6\left(\mathrm{~d}, \mathrm{C}_{4}\right), 63.5\left(\mathrm{t}, \mathrm{C}_{5}\right), 120.3\left(\mathrm{~d}, \mathrm{C}_{3^{\prime}}\right), 137.1\left(\mathrm{~d}, \mathrm{C}_{4^{\prime}}\right), 154.1\left(\mathrm{~s}, \mathrm{C}_{2}\right)$, $172.0\left(\mathrm{~s}, \mathrm{C}_{1^{\prime}}\right) ; \mathrm{MS}(\mathrm{EI}) \mathrm{m} / z$ (relative intensity) $225(\mathrm{M})^{+}(19), 210$ $(\mathrm{M}-\mathrm{Me})^{+}(1), 130(42), 96(\mathrm{M}-\mathrm{H}-\text { oxazolidin-2-one })^{+}(100)$; HRMS calcd for $\mathrm{C}_{12} \mathrm{H}_{19} \mathrm{NO}_{3}\left[(\mathrm{M})^{+}\right]$225.1365, found 225.1376.

(4R,5S)-3-[(E)-Hex-3-enoyl]-4-methyl-5-phenyloxazolidin-2-one (3i). Acid 4a $(0.3 \mathrm{~mL}, 2.46 \mathrm{mmol})$ and $(4 R, 5 S)-4-m e t h y l-5-$ phenyloxazolidin-2-one (523 mg, $2.95 \mathrm{mmol}, 1.2$ equiv) were subjected to the general procedure for the synthesis of $N$-acyl oxazolidin-2-ones 3 and yielded, after purification by flash chromatography (32 $\mathrm{cm}$ of height of silica gel, $n$-hexane/EtOAc 90:10), compound $3 \mathbf{i}(581 \mathrm{mg}, 87 \%)$ as a colorless oil: $R_{f}=0.19(n-$ hexane/EtOAc 80:20), 0.62 ( $n$-hexane/EtOAc 60:40); $[\alpha]^{25}{ }_{\mathrm{D}}+29.8$ (c 1.1, $\left.\mathrm{CHCl}_{3}\right) ;{ }^{1} \mathrm{H}$ NMR $\left(600 \mathrm{MHz}, \mathrm{CDCl}_{3}\right) \delta 0.89(\mathrm{~d}, J=6.7 \mathrm{~Hz}$, $\left.3 \mathrm{H}, \mathrm{CH}_{3} \mathrm{C}_{4}\right), 1.00\left(\mathrm{t}, J=7.4 \mathrm{~Hz}, 3 \mathrm{H}, \mathrm{H}_{6^{\prime}}\right), 2.04-2.10\left(\mathrm{~m}, 2 \mathrm{H}, \mathrm{H}_{5^{\prime}}\right)$, $3.63-3.71\left(\mathrm{~m}, 2 \mathrm{H}, \mathrm{H}_{2^{\prime}}\right), 4.75\left(\mathrm{dq}, J=6.7,6.7 \mathrm{~Hz}, 1 \mathrm{H}, \mathrm{H}_{4}\right), 5.57-5.61$ $\left(\mathrm{m}, 1 \mathrm{H}, \mathrm{H}_{5}\right), 5.65-5.70\left(\mathrm{~m}, 2 \mathrm{H}, \mathrm{H}_{3^{\prime}}, \mathrm{H}_{4^{\prime}}\right), 7.29-7.30(\mathrm{~m}, 2 \mathrm{H}, \mathrm{Ph})$, 7.35-7.38 (m, 1H, Ph), 7.40-7.42 (m, 2H, Ph); ${ }^{13} \mathrm{C}$ NMR $(150$

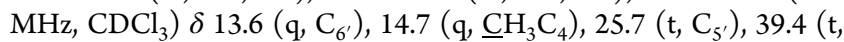
$\left.\mathrm{C}_{2^{\prime}}\right), 54.9\left(\mathrm{~d}, \mathrm{C}_{4}\right), 79.1\left(\mathrm{~d}, \mathrm{C}_{5}\right), 120.1\left(\mathrm{~d}, \mathrm{C}_{3^{\prime}}\right), 125.8(\mathrm{~d}, 2 \mathrm{C}, \mathrm{Ph})$, 128.8 (d, 2C, Ph), 128.9 (d, Ph), $133.4(\mathrm{~s}, \mathrm{Ph}), 137.1\left(\mathrm{~d}, \mathrm{C}_{4^{\prime}}\right), 153.1$ $\left(\mathrm{s}, \mathrm{C}_{2}\right), 171.8\left(\mathrm{~s}, \mathrm{C}_{1^{\prime}}\right)$; HRMS calcd for $\mathrm{C}_{16} \mathrm{H}_{19} \mathrm{NO}_{3} \mathrm{Na}\left[(\mathrm{M}+\mathrm{Na})^{+}\right]$ 296.1263, found 296.1261 .

General Procedure for the Synthesis of syn-Aldols 2. All the subsequent operations were performed under an Ar atmosphere. A solution of the $\mathrm{N}$-acyl oxazolidin-2-ones in dry DCM (1 M) was cooled to $-78{ }^{\circ} \mathrm{C}$. TEA ( 1.3 equiv) and a $1 \mathrm{M}$ solution of $n$-Bu $\mathrm{Bu}_{2} \mathrm{BOTf}$ in DCM (1.2 equiv) were dropped sequentially, and then the mixture was stirred at that temperature for $30 \mathrm{~min}$. After that, it was warmed to $0{ }^{\circ} \mathrm{C}$, and after $20 \mathrm{~min}$, it was recooled to $-78{ }^{\circ} \mathrm{C}$, the aldehyde $\mathrm{R}^{2} \mathrm{CHO}$ ( 1.5 equiv) was added, and the mixture was allowed to warm to rt. After $15 \mathrm{~h}$, the mixture was cooled to $0{ }^{\circ} \mathrm{C}$ and subjected to an oxidative workup. To it were sequentially added a $\mathrm{pH} 7$ buffer solution $(1.1 \mathrm{~mL} / \mathrm{mmol}$ of $\mathrm{N}$-acyl oxazolidin-2-ones), $\mathrm{MeOH}(2.6$ $\mathrm{mL} / \mathrm{mmol}$ of $\mathrm{N}$-acyl oxazolidin-2-ones), and a $35 \mathrm{wt} \%$ solution of $\mathrm{H}_{2} \mathrm{O}_{2}$ in water ( $1.1 \mathrm{~mL} / \mathrm{mmol}$ of $\mathrm{N}$-acyl oxazolidin-2-ones). The layers were then separated, and the aqueous layer was extracted three times with DCM. The combined organic layers were dried over anhydrous $\mathrm{MgSO}_{4}$, filtered, and concentrated. A non-aqueous simplified workup is also valid. A small amount of silica gel 60 (35-70 mesh) was added; the solvent was removed in the rotavap, and the silica-supported crude was purified. The crude was purified by flash chromatography (the homoallylic alcohol is usually slightly more polar than the starting $\mathrm{N}$-acyl oxazolidin-2-ones) to yield desired compounds. Stored under $\mathrm{Ar}$ at $-18^{\circ} \mathrm{C}$, aldols were stable for at least 12 months. Except for anti-aldol 2a, all the syn-aldols 2 were prepared as described above. anti-Aldol $2 \mathrm{a}$ and syn-aldols $2 \mathrm{a}-\mathbf{2 g}$ were described in our previous publication. ${ }^{30}$

$3-\left\{\left(R^{*}, E\right)-2-\left[\left(R^{*}\right)-(4-B r o m o p h e n y l)(h y d r o x y)\right.\right.$ methyl]hex-3-enoyl\}oxazolidin-2-one (2h). N-Acyl oxazolidin-2-one 3a (994 mg, 5.43 $\mathrm{mmol}$ ) was subjected to the general procedure for the synthesis of syn-aldols 2 and yielded, after purification by flash chromatography (17 cm of height of silica gel, $n$-hexane/EtOAc 70:30), compound $\mathbf{2 h}$ $(1.18 \mathrm{~g}, 59 \%)$ as a white solid: $R_{f}=0.41$ ( $n$-hexane/EtOAc 60:40, two times); mp $60-64{ }^{\circ} \mathrm{C}$ (from DCM $/ n$-hexane); ${ }^{1} \mathrm{H}$ NMR $(500 \mathrm{MHz}$, $\left.\mathrm{CDCl}_{3}\right) \delta 0.94\left(\mathrm{t}, J=7.5 \mathrm{~Hz}, 3 \mathrm{H}, \mathrm{H}_{6^{\prime}}\right), 2.00-2.06\left(\mathrm{~m}, 2 \mathrm{H}, \mathrm{H}_{5^{\prime}}\right), 3.09$ (br s, $1 \mathrm{H}, \mathrm{OH}), 3.85-3.91\left(\mathrm{~m}, 1 \mathrm{H}, \mathrm{H}_{4}\right), 3.93-3.99\left(\mathrm{~m}, 1 \mathrm{H}, \mathrm{H}_{4}\right)$, $4.26-4.31\left(\mathrm{~m}, 1 \mathrm{H}, \mathrm{H}_{5}\right), 4.32-4.39\left(\mathrm{~m}, 1 \mathrm{H}, \mathrm{H}_{5}\right), 4.74(\mathrm{dd}, J=9.1,5.7$ $\left.\mathrm{Hz}, 1 \mathrm{H}, \mathrm{H}_{2^{\prime}}\right), 4.99\left(\mathrm{~d}, J=5.7 \mathrm{~Hz}, 1 \mathrm{H}, \mathrm{H}_{1^{\prime \prime}}\right), 5.52(\mathrm{dd}, J=15.5,9.1 \mathrm{~Hz}$, $\left.1 \mathrm{H}, \mathrm{H}_{3^{\prime}}\right), 5.69\left(\mathrm{dt}, J=15.5,6.1 \mathrm{~Hz}, 1 \mathrm{H}, \mathrm{H}_{4^{\prime}}\right), 7.24(\mathrm{~d}, J=8.5 \mathrm{~Hz}, 2 \mathrm{H}$, Ar), 7.44 (d, $J=8.5 \mathrm{~Hz}, 2 \mathrm{H}, \mathrm{Ar}) ;{ }^{13} \mathrm{C}$ NMR $\left(150 \mathrm{MHz}, \mathrm{CDCl}_{3}\right) \delta$ $13.4\left(\mathrm{q}, \mathrm{C}_{6^{\prime}}\right), 25.9\left(\mathrm{t}, \mathrm{C}_{5^{\prime}}\right), 42.7\left(\mathrm{t}, \mathrm{C}_{4}\right), 53.8\left(\mathrm{~d}, \mathrm{C}_{2^{\prime}}\right), 62.0\left(\mathrm{t}, \mathrm{C}_{5}\right)$, 73.9 (d, $\mathrm{C}_{1^{\prime \prime}}$ ), 121.3 (d, $\mathrm{C}_{3^{\prime}}$ ), 121.7 (s, Ar), 128.7 (d, 2C, Ar), 131.3 (d, 2C, Ar), 139.8 (s, Ar), 140.7 (d, $\mathrm{C}_{4^{\prime}}$ ), 153.0 (s, C 2 ), 173.7 (s, $\left.\mathrm{C}_{1^{\prime}}\right)$; HRMS calcd for $\mathrm{C}_{16} \mathrm{H}_{18}{ }^{79} \mathrm{BrNO}_{4} \mathrm{Na}\left[(\mathrm{M}+\mathrm{Na})^{+}\right]$390.0317, found 390.0314 .

(R)-4-Benzyl-3-\{(R,E)-2-[(S)-1-hydroxyethyl]hex-3-enoyl\}oxazolidin-2-one (2i). N-Acyl oxazolidin-2-one $3 \mathrm{~g}$ (643 mg, 2.35 $\mathrm{mmol}$ ) was subjected to the general procedure for the synthesis of syn-aldols 2 and yielded, after purification by flash chromatography (18 $\mathrm{cm}$ of height of silica gel, $n$-hexane/EtOAc 70:30), compound $2 \mathbf{i}$ $(559 \mathrm{mg}, 75 \%)$ as a colorless oil: $R_{f}=0.42(n$-hexane/EtOAc 60:40); $[\alpha]_{\mathrm{D}}^{25} \mathrm{O}\left(c 1.0, \mathrm{CHCl}_{3}\right),-22.0\left(c 1.7, \mathrm{Et}_{2} \mathrm{O}\right) ;{ }^{1} \mathrm{H}$ NMR $(500 \mathrm{MHz}$, $\left.\mathrm{CDCl}_{3}\right) \delta 1.04\left(\mathrm{t}, J=7.5 \mathrm{~Hz}, 3 \mathrm{H}, \mathrm{H}_{6^{\prime}}\right), 1.19\left(\mathrm{~d}, J=6.4 \mathrm{~Hz}, 3 \mathrm{H}, \mathrm{H}_{2^{\prime \prime}}\right)$, $2.11-2.17\left(\mathrm{~m}, 2 \mathrm{H}, \mathrm{H}_{5^{\prime}}\right), 2.79\left(\mathrm{dd}, J=13.4,9.2 \mathrm{~Hz}, 1 \mathrm{H}, \mathrm{CH}_{2} \mathrm{Ph}\right), 2.99$ (br s, $1 \mathrm{H}, \mathrm{OH}), 3.19$ (dd, $\left.J=13.5,2.9 \mathrm{~Hz}, 1 \mathrm{H}, \mathrm{C}_{2} \mathrm{Ph}\right), 4.13-4.19$ $\left(\mathrm{m}, 2 \mathrm{H}, 1 \times \mathrm{H}_{5}, \mathrm{H}_{1^{\prime \prime}}\right), 4.20-4.25\left(\mathrm{~m}, 1 \mathrm{H}, 1 \times \mathrm{H}_{5}\right), 4.44(\mathrm{dd}, J=9.2$, $\left.3.9 \mathrm{~Hz}, 1 \mathrm{H}, \mathrm{H}_{2^{\prime}}\right), 4.70-4.76\left(\mathrm{~m}, 1 \mathrm{H}, \mathrm{H}_{4}\right), 5.60(\mathrm{dd}, J=15.3,9.3 \mathrm{~Hz}$, $\left.1 \mathrm{H}, \mathrm{H}_{3^{\prime}}\right), 5.90\left(\mathrm{dt}, J=15.4,6.9 \mathrm{~Hz}, 1 \mathrm{H}, \mathrm{H}_{4^{\prime}}\right), 7.17-7.21(\mathrm{~m}, 2 \mathrm{H}, \mathrm{Ph})$, 7.27-7.29 (m, $1 \mathrm{H}, \mathrm{Ph}), 7.30-7.34(\mathrm{~m}, 2 \mathrm{H}, \mathrm{Ph}) ;{ }^{13} \mathrm{C}$ NMR $(125$ $\left.\mathrm{MHz}, \mathrm{CDCl}_{3}\right) \delta 13.7\left(\mathrm{q}, \mathrm{C}_{6^{\prime}}\right), 20.0\left(\mathrm{q}, \mathrm{C}_{2^{\prime \prime}}\right), 26.0\left(\mathrm{t}, \mathrm{C}_{5^{\prime}}\right), 37.7(\mathrm{t}$, $\left.\underline{\mathrm{CH}}_{2} \mathrm{Ph}\right), 52.5\left(\mathrm{~d}, \mathrm{C}_{2^{\prime}}\right), 55.1\left(\mathrm{~d}, \mathrm{C}_{4}\right), 66.0\left(\mathrm{t}, \mathrm{C}_{5}\right), 68.2\left(\mathrm{~d}, \mathrm{C}_{1^{\prime \prime}}\right), 121.6$ $\left(\mathrm{d}, \mathrm{C}_{3^{\prime}}\right), 127.6(\mathrm{~d}, \mathrm{Ph}), 129.1(\mathrm{~d}, 2 \mathrm{C}, \mathrm{Ph}), 129.6(\mathrm{~d}, 2 \mathrm{C}, \mathrm{Ph}), 135.1(\mathrm{~s}$ $\mathrm{Ph}), 140.2\left(\mathrm{~d}, \mathrm{C}_{4^{\prime}}\right), 153.1\left(\mathrm{~s}, \mathrm{C}_{2}\right), 174.8\left(\mathrm{~s}, \mathrm{C}_{1^{\prime}}\right)$; HRMS calcd for $\mathrm{C}_{18} \mathrm{H}_{23} \mathrm{NO}_{4} \mathrm{Na}\left[(\mathrm{M}+\mathrm{Na})^{+}\right] 340.1525$, found 340.1520 .

(R)-4-Benzyl-3-\{(R,E)-2-[(S)-1-hydroxy-3-phenylpropyl]hex-3enoyl\}oxazolidin-2-one (2j). $\mathrm{N}$-Acyl oxazolidine-2-one $3 \mathrm{~g}$ (600 mg, $2.19 \mathrm{mmol}$ ) was subjected to the general procedure for the synthesis of $s y n$-aldols 2 and yielded, after purification by flash chromatography (30 $\mathrm{cm}$ of height of silica gel, $n$-hexane/EtOAc 80:20), compound $\mathbf{2 j}$ (533 mg, 60\%) as a yellow oil: $R_{f}=0.39$ ( $n$-hexane/EtOAc 70:30); 
$[\alpha]_{\mathrm{D}}^{25}-13.2\left(c 1.0, \mathrm{CHCl}_{3}\right) ;{ }^{1} \mathrm{H}$ NMR $\left(500 \mathrm{MHz}, \mathrm{CDCl}_{3}\right) \delta 1.03(\mathrm{t}$, $\left.J=7.5 \mathrm{~Hz}, 3 \mathrm{H}, \mathrm{H}_{6^{\prime}}\right), 1.63($ br s, $1 \mathrm{H}, \mathrm{OH}), 1.68-1.75\left(\mathrm{~m}, 1 \mathrm{H}, \mathrm{H}_{2^{\prime \prime}}\right)$, $1.82-1.90\left(\mathrm{~m}, 1 \mathrm{H}, \mathrm{H}_{2^{\prime \prime}}\right), 2.10-2.16\left(\mathrm{~m}, 2 \mathrm{H}, \mathrm{H}_{5^{\prime}}\right), 2.66-2.72(\mathrm{~m}, 1 \mathrm{H}$, $\left.\mathrm{H}_{3^{\prime \prime}}\right), 2.78\left(\mathrm{~d}, J=13.5,9.0 \mathrm{~Hz}, 1 \mathrm{H}, \mathrm{C}_{4} \mathrm{C}_{2} \mathrm{Ph}\right), 2.81-2.87(\mathrm{~m}, 1 \mathrm{H}$, $\mathrm{H}_{3^{\prime \prime}}$ ), 3.20 (dd, $J=13.5,3.5 \mathrm{~Hz}, 1 \mathrm{H}, \mathrm{C}_{4} \mathrm{CH}_{2} \mathrm{Ph}$ ), $3.97(\mathrm{dt}, J=9.1,3.6$ $\left.\mathrm{Hz}, 1 \mathrm{H}, \mathrm{H}_{1^{\prime \prime}}\right), 4.14-4.22\left(\mathrm{~m}, 2 \mathrm{H}, \mathrm{H}_{5}\right), 4.50(\mathrm{dd}, J=9.3,3.9 \mathrm{~Hz}, 1 \mathrm{H}$, $\left.\mathrm{H}_{2^{\prime}}\right), 4.68-4.73\left(\mathrm{~m}, 1 \mathrm{H}, \mathrm{H}_{4}\right), 5.61(\mathrm{ddt}, J=15.4,9.2,1.4 \mathrm{~Hz}, 1 \mathrm{H}$, $\left.\mathrm{H}_{3^{\prime}}\right), 5.92\left(\mathrm{dt}, J=15.4,6.4 \mathrm{~Hz}, 1 \mathrm{H}, \mathrm{H}_{4^{\prime}}\right), 7.17-7.22(\mathrm{~m}, 5 \mathrm{H}, \mathrm{Ph})$, 7.27-7.34 (m, 5H, Ph); ${ }^{13} \mathrm{C}$ NMR $\left(150 \mathrm{MHz}, \mathrm{CDCl}_{3}\right) \delta 13.7(\mathrm{q}$, $\left.\mathrm{C}_{6^{\prime}}\right), 26.1\left(\mathrm{t}, \mathrm{C}_{5^{\prime}}\right), 32.0\left(\mathrm{t}, \mathrm{C}_{3^{\prime \prime}}\right), 35.8\left(\mathrm{t}, \mathrm{C}_{2^{\prime \prime}}\right), 37.7\left(\mathrm{t}, \mathrm{C}_{4} \underline{\mathrm{CH}_{2}} \mathrm{Ph}\right), 51.2$ $\left(\mathrm{d}, \mathrm{C}_{2^{\prime}}\right), 55.1\left(\mathrm{~d}, \mathrm{C}_{4}\right), 66.0\left(\mathrm{t}, \mathrm{C}_{\mathrm{s}}\right), 71.1\left(\mathrm{~d}, \mathrm{C}_{1^{\prime \prime}}\right), 121.3\left(\mathrm{~d}, \mathrm{C}_{3^{\prime}}\right), 125.9$ (d, Ph), 127.6 (d, Ph), 128.5 (d, 2C, Ph), 128.7 (d, 2C, Ph), 129.1 (d, 2C, Ph), 129.6 (d, 2C, Ph), 135.1 (s, Ph), $140.3\left(\mathrm{~d}, \mathrm{C}_{4^{\prime}}\right.$ ), 142.1 (s, $\mathrm{Ph}), 153.0\left(\mathrm{~s}, \mathrm{C}_{2}\right), 175.0\left(\mathrm{~s}, \mathrm{C}_{1^{\prime}}\right)$; HRMS calcd for $\mathrm{C}_{25} \mathrm{H}_{29} \mathrm{NO}_{4} \mathrm{Na}$ $\left[(\mathrm{M}+\mathrm{Na})^{+}\right]$430.1994, found 430.1998.

(S)-3-\{(S,E)-2-[(R)-1-Hydroxyethyl]hex-3-enoyl\}-4-isopropyloxazolidin-2-one (2k). N-Acyl oxazolidin-2-one $3 \mathrm{~h}(276 \mathrm{mg}, 1.23 \mathrm{mmol})$ was subjected to the general procedure for the synthesis of syn-aldols 2 and yielded, after purification by flash chromatography $(25 \mathrm{~cm}$ of height of silica gel, $n$-hexane/EtOAc 70:30), compound $2 \mathrm{k}(283 \mathrm{mg}$, $86 \%)$ as a thick colorless oil: $R_{f}=0.21$ ( $n$-hexane/EtOAc 70:30); $[\alpha]^{25}{ }_{\mathrm{D}}-27.8\left(c 1.1, \mathrm{CHCl}_{3}\right) ;{ }^{1} \mathrm{H} \mathrm{NMR}\left(500 \mathrm{MHz}, \mathrm{CDCl}_{3}\right) \delta 0.81(\mathrm{~d}$, $\left.J=6.9 \mathrm{~Hz}, 3 \mathrm{H},\left(\mathrm{CH}_{3}\right)_{2} \mathrm{CHC}_{4}\right), 0.88(\mathrm{~d}, J=6.9 \mathrm{~Hz}, 3 \mathrm{H}$, $\left.\left(\mathrm{CH}_{3}\right)_{2} \mathrm{CHC}_{4}\right), 0.96\left(\mathrm{t}, J=7.5 \mathrm{~Hz}, 3 \mathrm{H}, \mathrm{H}_{6^{\prime}}\right), 1.14(\mathrm{~d}, J=6.4 \mathrm{~Hz}$, $\left.3 \mathrm{H}, \mathrm{H}_{2^{\prime \prime}}\right), 2.03-2.08\left(\mathrm{~m}, 2 \mathrm{H}, \mathrm{H}_{5^{\prime}}\right), 2.25-2.34(\mathrm{~m}, 1 \mathrm{H}$, $\left.\left(\mathrm{CH}_{3}\right)_{2} \mathrm{CHC}_{4}\right), 3.10$ (br s, $\left.1 \mathrm{H}, \mathrm{OH}\right), 4.09-4.14\left(\mathrm{~m}, 1 \mathrm{H}, \mathrm{H}_{1^{\prime \prime}}\right), 4.17$ (dd, $\left.J=9.1,3.3 \mathrm{~Hz}, 1 \mathrm{H}, \mathrm{H}_{5}\right), 4.25\left(\mathrm{dd}, J=8.9,8.9 \mathrm{~Hz}, 1 \mathrm{H}, \mathrm{H}_{5}\right)$, 4.43-4.48 (m, $\left.2 \mathrm{H}, \mathrm{H}_{4}, \mathrm{H}_{2^{\prime}}\right), 5.52(\mathrm{ddq}, J=15.6,9.2,1.3 \mathrm{~Hz}, 1 \mathrm{H}$, $\left.\mathrm{H}_{3^{\prime}}\right), 5.87\left(\mathrm{dt}, J=15.0,6.5 \mathrm{~Hz}, 1 \mathrm{H}, \mathrm{H}_{4^{\prime}}\right) ;{ }^{13} \mathrm{C} \mathrm{NMR}(125 \mathrm{MHz}$, $\left.\mathrm{CDCl}_{3}\right) \delta 13.6\left(\mathrm{q}, \mathrm{C}_{6^{\prime}}\right), 14.6\left(\mathrm{q}, \quad\left(\underline{\mathrm{CH}}_{3}\right)_{2} \mathrm{CHC}_{4}\right), 17.9(\mathrm{q}$, $\left.\left(\mathrm{CH}_{3}\right)_{2} \mathrm{CHC}_{4}\right), 19.9\left(\mathrm{q}, \mathrm{C}_{2^{\prime \prime}}\right), 25.9\left(\mathrm{t}, \mathrm{C}_{5^{\prime}}\right), 28.3\left(\mathrm{~d},\left(\mathrm{CH}_{3}\right)_{2} \mathrm{CHC}_{4}\right)$, $52.2\left(\mathrm{~d}, \mathrm{C}_{2^{\prime}}\right), 58.2\left(\mathrm{~d}, \mathrm{C}_{4}\right), 63.2\left(\mathrm{t}, \mathrm{C}_{5}\right), 67.8\left(\mathrm{~d}, \mathrm{C}_{1^{\prime \prime}}\right), 121.7\left(\mathrm{~d}, \mathrm{C}_{3^{\prime}}\right)$, $140.1\left(\mathrm{~d}, \mathrm{C}_{4^{\prime}}\right), 153.6\left(\mathrm{~s}, \mathrm{C}_{2}\right), 175.1\left(\mathrm{~s}, \mathrm{C}_{1^{\prime}}\right) ; \mathrm{MS}(\mathrm{EI}) \mathrm{m} / \mathrm{z}$ (relative intensity) $225(\mathrm{M}-\mathrm{H}-i \text {-Pr })^{+}(1), 141(\mathrm{M}-\text { oxazolidin-2-one })^{+}$ (1), 128 (oxazolidin-2-one) ${ }^{+}(100), 113(\mathrm{M}-\mathrm{N}$-acyl oxazolidin-2one $)^{+}$(38); HRMS calcd for $\mathrm{C}_{11} \mathrm{H}_{15} \mathrm{NO}_{4}\left[(\mathrm{M}-\mathrm{H}-i \text {-Pr })^{+}\right]$ 225.1001, found 225.1007 .

(S)-3-\{(S,E)-2-[(R)-1-Hydroxy-3-phenylpropyl]hex-3-enoyl\}-4-isopropyloxazolidin-2-one (2l). N-Acyl oxazolidin-2-one 3h $(194 \mathrm{mg}$, $0.86 \mathrm{mmol}$ ) was subjected to the general procedure for the synthesis of $s y n$-aldols 2 and yielded, after purification by flash chromatography ( $25 \mathrm{~cm}$ of height of silica gel, $n$-hexane/EtOAc 80:20), compound 21 (215 mg, 69\%) as an amorphous white solid: $R_{f}=0.33$ ( $n$-hexane/ EtOAc 70:30); $[\alpha]^{25}-23.8\left(c\right.$ 1.1, $\left.\mathrm{CHCl}_{3}\right) ;{ }^{1} \mathrm{H}$ NMR $(500 \mathrm{MHz}$, $\left.\mathrm{CDCl}_{3}\right) \delta 0.80\left(\mathrm{~d}, J=7.0 \mathrm{~Hz}, 3 \mathrm{H},\left(\mathrm{CH}_{3}\right)_{2} \mathrm{CHC}_{4}\right), 0.87(\mathrm{~d}, J=7.0 \mathrm{~Hz}$, $\left.3 \mathrm{H},\left(\mathrm{CH}_{3}\right)_{2} \mathrm{CHC}_{4}\right), 0.95\left(\mathrm{t}, J=7.5 \mathrm{~Hz}, 3 \mathrm{H}, \mathrm{H}_{6^{\prime}}\right), 1.63-1.70(\mathrm{~m}, 1 \mathrm{H}$, $\left.\mathrm{H}_{2^{\prime \prime}}\right), 1.78-1.86\left(\mathrm{~m}, 1 \mathrm{H}, \mathrm{H}_{2^{\prime \prime}}\right), 2.02-2.08\left(\mathrm{~m}, 2 \mathrm{H}, \mathrm{H}_{5^{\prime}}\right), 2.25-2.34$ $\left(\mathrm{m}, 1 \mathrm{H},\left(\mathrm{CH}_{3}\right)_{2} \mathrm{CHC}_{4}\right), 2.63-2.69\left(\mathrm{~m}, 1 \mathrm{H}, \mathrm{H}_{3^{\prime \prime}}\right), 2.77-2.83(\mathrm{~m}, 1 \mathrm{H}$, $\mathrm{H}_{3^{\prime \prime}}$ ), 3.21 (br s, $\left.1 \mathrm{H}, \mathrm{OH}\right), 3.91-3.95\left(\mathrm{~m}, 1 \mathrm{H}, \mathrm{H}_{1^{\prime \prime}}\right), 4.16(\mathrm{dd}, J=9.2$, $\left.3.2 \mathrm{~Hz}, 1 \mathrm{H}, \mathrm{H}_{5}\right), 4.23\left(\mathrm{dd}, J=8.7,8.7 \mathrm{~Hz}, 1 \mathrm{H}, \mathrm{H}_{5}\right), 4.43(\mathrm{dt}, J=8.3$, $\left.3.4 \mathrm{~Hz}, 1 \mathrm{H}, \mathrm{H}_{4}\right), 4.52\left(\mathrm{dd}, J=9.2,3.4 \mathrm{~Hz}, 1 \mathrm{H}, \mathrm{H}_{2^{\prime}}\right), 5.54(\mathrm{ddt}, J=$ 15.6, 9.2, $\left.1.5 \mathrm{~Hz}, 1 \mathrm{H}, \mathrm{H}_{3^{\prime}}\right), 5.88\left(\mathrm{dt}, J=15.4,6.5 \mathrm{~Hz}, 1 \mathrm{H}, \mathrm{H}_{4^{\prime}}\right) ;{ }^{13} \mathrm{C}$ NMR $\left(125 \mathrm{MHz}, \mathrm{CDCl}_{3}\right) \delta 13.7\left(\mathrm{q}, \mathrm{C}_{6^{\prime}}\right), 14.7\left(\mathrm{q},\left(\mathrm{CH}_{3}\right)_{2} \mathrm{CHC}_{4}\right)$, $\left.18.0\left(\mathrm{q},\left(\mathrm{CH}_{3}\right)_{2} \mathrm{CHC}_{4}\right), 26.0\left(\mathrm{t}, \mathrm{C}_{5^{\prime}}\right), 28.3\left(\mathrm{~d},\left(\mathrm{CH}_{3}\right)_{2} \underline{\mathrm{CHC}}\right)_{4}\right), 32.0(\mathrm{t}$, $\left.\mathrm{C}_{3^{\prime \prime}}\right), 35.8\left(\mathrm{t}, \mathrm{C}_{2^{\prime \prime}}\right), 50.9\left(\mathrm{~d}, \mathrm{C}_{2^{\prime}}\right), 58.2\left(\mathrm{~d}, \mathrm{C}_{4}\right), 63.2\left(\mathrm{t}, \mathrm{C}_{5}\right), 70.7(\mathrm{~d}$, $\left.\mathrm{C}_{1^{\prime \prime}}\right), 121.5\left(\mathrm{~d}, \mathrm{C}_{3^{\prime}}\right), 125.9(\mathrm{~d}, \mathrm{Ph}), 128.5(\mathrm{~d}, 2 \mathrm{C}, \mathrm{Ph}), 128.7$ (d, 2C, $\mathrm{Ph}), 140.2\left(\mathrm{~d}, \mathrm{C}_{4^{\prime}}\right), 142.1(\mathrm{~s}, \mathrm{Ph}), 153.5\left(\mathrm{~s}, \mathrm{C}_{2}\right), 175.4\left(\mathrm{~s}, \mathrm{C}_{1^{\prime}}\right) ; \mathrm{MS}$ (EI) $m / z$ (relative intensity) $359(\mathrm{M})^{+}(1), 316(\mathrm{M}-i-\mathrm{Pr})^{+}(1), 225$

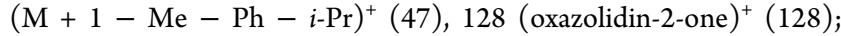
HRMS calcd for $\mathrm{C}_{21} \mathrm{H}_{29} \mathrm{NO}_{4}\left[(\mathrm{M})^{+}\right]$359.2097, found 359.2111.

(4R,5S)-3-\{(2R,3S)-2-[(E)-But-1-en-1-yl]3-hydroxyheptanoyl\}-4methyl-5-phenyloxazolidin-2-one ( $2 m)$. $N$-Acyl oxazolidin-2-one $3 \mathbf{i}$ $(207 \mathrm{mg}, 0.76 \mathrm{mmol}$ ) was subjected to the general procedure for the synthesis of syn-aldols 2 and yielded, after purification by flash chromatography $(21 \mathrm{~cm}$ of height of silica gel, $n$-hexane/EtOAc $85: 15)$, compound $2 \mathrm{~m}(175 \mathrm{mg}, 64 \%)$ as a thick colorless oil: $R_{f}=$ 0.33 (n-hexane/EtOAc 80:20); $[\alpha]_{\mathrm{D}}^{25}+75.3$ (c 1.0, $\left.\mathrm{CHCl}_{3}\right) ;{ }^{1} \mathrm{H}$ NMR $\left(500 \mathrm{MHz}, \mathrm{CDCl}_{3}\right) \delta 0.84-0.88\left(\right.$ br m, 3H, $\left.\mathrm{CH}_{3} \mathrm{C}_{4}\right), 0.89-$ 0.94 (br m, 3H, $\mathrm{H}_{5^{\prime \prime}}$ ), 0.97-1.03 (br m, 3H, $\mathrm{H}_{6^{\prime}}$ ), 1.29-1.39 (br m, $\left.3 \mathrm{H}, 3 \times \mathrm{C}_{1^{\prime \prime}}\left(\mathrm{CH}_{2}\right)_{3} \mathrm{CH}_{3}\right), 1.42-1.55\left(\right.$ br m, $\left.3 \mathrm{H}, 3 \times \mathrm{C}_{1^{\prime \prime}}\left(\mathrm{C}_{2}\right)_{3} \mathrm{CH}_{3}\right)$,
2.03-2.13 (br m, $2 \mathrm{H}, \mathrm{H}_{5^{\prime}}$ ), 3.01 (br s, $\left.1 \mathrm{H}, \mathrm{OH}\right), 3.96$ (br m, $1 \mathrm{H}$, $\mathrm{H}_{1^{\prime \prime}}$ ), 4.45-4.51 (br m, $1 \mathrm{H}, \mathrm{H}_{2^{\prime}}$ ), 4.77-4.85 (br m, $1 \mathrm{H}, \mathrm{H}_{4}$ ), 5.535.61 (br m, $\left.1 \mathrm{H}, \mathrm{H}_{3^{\prime}}\right)$, 5.65-5.69 (br m, $\left.1 \mathrm{H}, \mathrm{H}_{5}\right), 5.82-5.89$ (br m, $\left.1 \mathrm{H}, \mathrm{H}_{4^{\prime}}\right), 7.27-7.32(\mathrm{~m}, 2 \mathrm{H}, \mathrm{Ph}), 7.35-7.44(\mathrm{~m}, 3 \mathrm{H}, \mathrm{Ph}) ;{ }^{13} \mathrm{C} \mathrm{NMR}$ $\left(125 \mathrm{MHz}, \mathrm{CDCl}_{3}\right) \delta 13.7\left(\mathrm{q}, \mathrm{C}_{6^{\prime}}\right), 14.1\left(\mathrm{q}, \mathrm{C}_{5^{\prime \prime}}\right), 14.4\left(\mathrm{q}, \mathrm{CH}_{3} \mathrm{C}_{4}\right)$, $22.7\left(t, C_{4^{\prime \prime}}\right), 26.0\left(t, C_{5^{\prime}}\right), 28.0\left(t, C_{3^{\prime \prime}}\right), 33.9\left(t, C_{2^{\prime \prime}}\right), 51.3\left(\mathrm{~d}, \mathrm{C}_{2^{\prime}}\right)$, $54.8\left(\mathrm{~d}, \mathrm{C}_{4}\right), 72.0$ (d, $\left.\mathrm{C}_{1^{\prime \prime}}\right), 78.9\left(\mathrm{~d}, \mathrm{C}_{5}\right), 121.4\left(\mathrm{~d}, \mathrm{C}_{3^{\prime}}\right), 125.8$ (d, 2C, $\mathrm{Ph}), 128.9(\mathrm{~d}, 2 \mathrm{C}, \mathrm{Ph}), 129.0(\mathrm{~d}, \mathrm{Ph}), 133.4(\mathrm{~s}, \mathrm{Ph}), 139.6\left(\mathrm{~d}, \mathrm{C}_{4^{\prime}}\right)$, $152.7\left(\mathrm{~s}, \mathrm{C}_{2}\right), 174.9\left(\mathrm{~s}, \mathrm{C}_{1^{\prime}}\right)$; HRMS calcd for $\mathrm{C}_{21} \mathrm{H}_{29} \mathrm{NO}_{4} \mathrm{Na}[(\mathrm{M}+$ $\left.\mathrm{Na})^{+}\right]$382.1994, found 382.1995 .

General Procedure for the Synthesis of Bicycles 5. With aldols 2 as a starting point (two-step EAP), to a solution of the homoallylic alcohol and the aldehyde $\mathrm{R}^{3} \mathrm{CHO}$ (1.5 equiv) in dry DCM $(0.1 \mathrm{M})$ was added, under an $\mathrm{Ar}$ atmosphere, $\mathrm{BF}_{3} \cdot \mathrm{OEt}_{2}$ (2.5 equiv). Once TLC analysis showed full conversion $(<30 \mathrm{~min})$, the reaction was quenched with $\mathrm{H}_{2} \mathrm{O}$. The layers were separated, and the aqueous layer was extracted three times with DCM. The combined organic layers were dried over anhydrous $\mathrm{MgSO}_{4}$, filtered, and concentrated. ${ }^{76}$ The crude was purified by flash chromatography (the bicycle is usually slightly more apolar than the starting homoallylic alcohol) to yield the desired product. With $\mathrm{N}$-acyl oxazolidin-2-ones 3 as a starting point (one-pot EAP), a solution of the $\mathrm{N}$-acyl oxazolidin2 -one in dry DCM $(1 \mathrm{M})$ was cooled to $-78{ }^{\circ} \mathrm{C}$. TEA (1.3 equiv) and a $1 \mathrm{M}$ solution of $n$-Bu $\mathrm{Bu}_{2}$ BOTf in DCM (1.2 equiv) were added under an Ar atmosphere sequentially, and the mixture was stirred at that temperature for $30 \mathrm{~min}$. Then, it was warmed to $0^{\circ} \mathrm{C}$, and after $20 \mathrm{~min}$, it was recooled to $-78{ }^{\circ} \mathrm{C}$, the aldehyde $\mathrm{R}^{2} \mathrm{CHO}$ ( 1 equiv) was added, and the mixture was allowed to warm to rt. After $15 \mathrm{~h}$, the aldehyde $\mathrm{R}^{3} \mathrm{CHO}$ (1.5 equiv) and $\mathrm{BF}_{3} \cdot \mathrm{OEt}_{2}$ (2.5 equiv) were sequentially added under an $\mathrm{Ar}$ atmosphere. Once TLC analysis revealed full conversion $(<30 \mathrm{~min})$, the reaction was quenched and the mixture purified as described above. Traces of an UV-vis polar byproduct, the 2-oxonia-Cope rearranged isomer 6 , could be punctually detected. Bicycles $\mathbf{5}$ are highly stable and can be stored without an $\mathrm{Ar}$ atmosphere at rt without decomposition. Except for products $\mathbf{5 c - A c}, \mathbf{5 1}, \mathbf{5 r}, \mathbf{5 u}$, and $\mathbf{5 a g}-\mathbf{5 a i}$, the rest of bicycles $\mathbf{5}$ were described in our previous publication (see the Supporting Information for the correlation of the molecule numbering between both publications $){ }^{30}$

2-[(4aS,5S,7R, 8R,8aS)-5,7-Dibutyl-8-ethyl-2,4-dioxotetrahydro$2 \mathrm{H}, 5 \mathrm{H}$-pyrano[3,4-e][1,3]oxazin-3(4H)-yl]ethyl Acetate (5c-Ac). Aldol 2c $(38.7 \mathrm{mg}, 0.14 \mathrm{mmol})$ was subjectted to the general procedure for the synthesis of bicycles 5 (two-step EAP) using acetic acid $(1.4 \mathrm{~mL}, 0.1 \mathrm{M})$ as the solvent to yield, after purification by flash chromatography (20 cm of height of silica gel, $n$-hexane/EtOAc $80: 20)$, title compound $5 \mathrm{c}-\mathrm{Ac}(41 \mathrm{mg}, 71 \%,>95: 5 \mathrm{dr})$ as an amorphous white solid. $R_{f}=0.4$ ( $n$-hexane/EtOAc 60:40); ${ }^{1} \mathrm{H}$ NMR $\left(600 \mathrm{MHz}, \mathrm{CDCl}_{3}\right) \delta 0.87-0.97\left(\mathrm{~m}, 9 \mathrm{H}, \mathrm{H}_{2^{\prime \prime}}, \mathrm{H}_{4^{\prime}}, \mathrm{H}_{4^{\prime \prime}}\right), 1.26-1.79$ $\left(\mathrm{m}, 14 \mathrm{H}, \mathrm{H} 8,1 \times \mathrm{H}_{1}^{\prime}, 6 \times \mathrm{CH}_{2}\right), 2.01\left(\mathrm{~s}, 3 \mathrm{H}, \mathrm{OCOCH}_{3}\right), 2.28(\mathrm{br} \mathrm{s}$, $\left.1 \mathrm{H}, \mathrm{H}_{1^{\prime}}\right), 2.38$ (dd, $\left.J=11.1,11.1 \mathrm{~Hz}, 1 \mathrm{H}, \mathrm{H}_{4 \mathrm{a}}\right), 3.10(\mathrm{dd}, J=8.4,8.4$ $\mathrm{Hz}, 1 \mathrm{H}, \mathrm{H} 7), 3.41\left(\mathrm{t}, J=8.7 \mathrm{~Hz}, 1 \mathrm{H}, \mathrm{H}_{5}\right), 3.92-4.00(\mathrm{~m}, 1 \mathrm{H}$, $\left.\mathrm{NCH}_{2} \mathrm{CH}_{2} \mathrm{OCOCH}_{3}\right)$, 4.07-4.13 (m, $\left.1 \mathrm{H}, \mathrm{NCH}_{2} \mathrm{CH}_{2} \mathrm{OCOCH}_{3}\right)$, $4.17(\mathrm{t}, J=11.1 \mathrm{~Hz}, 1 \mathrm{H}, \mathrm{H} 8 \mathrm{a}), 4.27\left(\mathrm{br} \mathrm{s}, 2 \mathrm{H}, \mathrm{NCH}_{2} \mathrm{CH}_{2} \mathrm{OCOCH}_{3}\right)$; ${ }^{13} \mathrm{C}$ NMR $\left(150 \mathrm{MHz}, \mathrm{CDCl}_{3}\right) \delta 9.5\left(\mathrm{q}, \mathrm{C}_{2^{\prime \prime \prime}}\right), 14.2\left(\mathrm{q}, \mathrm{C}_{4^{\prime}}\right.$ or $\left.\mathrm{C}_{4^{\prime \prime}}\right)$, $14.2\left(\mathrm{q}, \mathrm{C}_{4^{\prime}}\right.$ or $\left.\mathrm{C}_{4^{\prime \prime}}\right), 18.6\left(\mathrm{t}, \mathrm{C}_{1^{\prime \prime \prime}}\right), 20.91\left(\mathrm{q}, \mathrm{NCH}_{2} \mathrm{CH}_{2} \mathrm{OCOCH}_{3}\right)$, $22.6\left(\mathrm{t}, \mathrm{C}_{3^{\prime}}\right.$ or $\left.\mathrm{C}_{3^{\prime \prime}}\right), 22.6\left(\mathrm{t}, \mathrm{C}_{2^{\prime}}\right.$ or $\left.\mathrm{C}_{2^{\prime \prime}}\right), 27.6\left(\mathrm{t}, \mathrm{C}_{2^{\prime}}\right.$ or $\left.\mathrm{C}_{2^{\prime \prime}}\right), 32.1(\mathrm{t}$, $\left.\mathrm{C}_{1^{\prime \prime}}\right), 34.0\left(\mathrm{t}, \mathrm{C}_{1^{\prime}}\right), 41.5\left(\mathrm{t}, \mathrm{NCH}_{2} \mathrm{CH}_{2} \mathrm{OCOCH}_{3}\right), 45.2\left(\mathrm{~d}, \mathrm{C}_{8}\right), 47.5$ $\left(\mathrm{d}, \mathrm{C}_{4 \mathrm{a}}\right), 61.6\left(\mathrm{t}, \mathrm{NCH}_{2} \mathrm{CH}_{2} \mathrm{OCOCH}_{3}\right), 74.7\left(\mathrm{~d}, \mathrm{C}_{5}\right), 76.7\left(\mathrm{~d}, \mathrm{C}_{8 \mathrm{a}}\right)$, $76.9\left(\mathrm{~d}, \mathrm{C}_{7}\right), 152.5\left(\mathrm{~s}, \mathrm{C}_{2}\right), 169.5\left(\mathrm{~s}, \mathrm{C}_{4}\right), 171.2\left(\mathrm{~s}, \mathrm{OCOCH}_{3}\right)$; HRMS calcd for $\mathrm{C}_{21} \mathrm{H}_{35} \mathrm{NO}_{6} \mathrm{Na}\left[(\mathrm{M}+\mathrm{Na})^{+}\right] 420.2362$, found 420.2361

$\left(4 a^{\prime} S^{*} 5^{\prime} S^{*}, 8^{\prime} S^{*}, 8 a^{\prime} S *\right)-8^{\prime}-$ Ethyl-3'-(2-hydroxyethyl)-5'-methyltetrahydro-2' H-spiro[cyclohexane-1,7'-pyrano[3,4-e][1,3]oxazine]$2^{\prime}, 4^{\prime}\left(3^{\prime} H\right)$-dione (5l). Aldol $2 \mathrm{~b}(30 \mathrm{mg}, 0.13 \mathrm{mmol})$ and cyclohexanone (0.03 mL, $0.29 \mathrm{mmol}, 2.2$ equiv) were subjected to the general procedure for the Prins cyclization and yielded, after purification by flash chromatography $(21 \mathrm{~cm}$ of height of silica gel, $n$-hexane/EtOAc 75:25), THF 81 (7.8 mg, 19\%, 80:20 dr), title compound $51(10 \mathrm{mg}, 23 \%,>95: 5 \mathrm{dr})$, and previously described bicycle $\mathbf{5 b}(8 \mathrm{mg}, 45 \%,>95: 5 \mathrm{dr})$. $\mathbf{5 1}$ was isolated as a colorless oil, 
and its description is given here: $R_{f}=0.44$ ( $n$-hexane/EtOAc 60:40, two times); ${ }^{1} \mathrm{H}$ NMR (500 MHz, $\left.\mathrm{CDCl}_{3}\right) \delta 1.11(\mathrm{t}, J=7.3 \mathrm{~Hz}, 3 \mathrm{H}$, $\left.\mathrm{H}_{2^{\prime \prime \prime}}\right), 1.11-1.17\left(\mathrm{~m}, 1 \mathrm{H}, \mathrm{C}_{2}\right.$ from cyclohexane), $1.21-1.26(\mathrm{~m}, 1 \mathrm{H}$, $\mathrm{CH}_{2}$ from cyclohexane), $1.31-1.39\left(\mathrm{~m}, 1 \mathrm{H}, \mathrm{CH}_{2}\right.$ from cyclohexane), 1.41-1.50 (m, $6 \mathrm{H}, \mathrm{H}_{8}, 3 \times \mathrm{CH}_{2}$ from cyclohexane, $\left.2 \times \mathrm{H}_{1^{\prime \prime \prime}}\right), 1.52(\mathrm{~d}$, $\left.J=6.0 \mathrm{~Hz}, 3 \mathrm{H}, \mathrm{H}_{1^{\prime}}\right), 1.65-1.70\left(\mathrm{~m}, 2 \mathrm{H}, \mathrm{CH}_{2}\right.$ from cyclohexane), 1.71-1.77 (m, $2 \mathrm{H}, \mathrm{C}_{2}$ from cyclohexane), $1.97(\mathrm{br} \mathrm{s}, 1 \mathrm{H}, \mathrm{OH}), 2.34$ $\left(\mathrm{dd}, J=12.3,9.9 \mathrm{~Hz}, 1 \mathrm{H}, \mathrm{H}_{4 \mathrm{a}}\right), 3.74\left(\mathrm{dq}, J=9.8,6.0 \mathrm{~Hz}, 1 \mathrm{H}, \mathrm{H}_{5}\right)$, 3.77-3.85 (br m, $2 \mathrm{H}, \mathrm{NCH}_{2} \mathrm{CH}_{2} \mathrm{OH}$ ), 3.89 (ddd, $J=14.1,6.0,4.2$ $\mathrm{Hz}, 1 \mathrm{H}, \mathrm{NC}_{2} \mathrm{CH}_{2} \mathrm{OH}$ ), 4.09 (ddd, $J=14.1,6.0,4.2 \mathrm{~Hz}, 1 \mathrm{H}$, $\mathrm{NCH}_{2} \mathrm{CH}_{2} \mathrm{OH}$ ), 4.31 (dd, $J=12.1,10.5 \mathrm{~Hz}, 1 \mathrm{H}, \mathrm{H}_{8 \mathrm{a}}$ ); ${ }^{13} \mathrm{C} \mathrm{NMR}$ $\left(125 \mathrm{MHz}, \mathrm{CDCl}_{3}\right) \delta 15.2$ (q, $\left.\mathrm{C}_{2^{\prime \prime \prime}}\right), 20.4\left(\mathrm{t}, \mathrm{CH}_{2}\right.$ from cyclohexane), $21.3\left(\mathrm{t}, \mathrm{CH}_{2}\right.$ from cyclohexane), $21.4\left(\mathrm{q}, \mathrm{C}_{1^{\prime}}\right), 21.7\left(\mathrm{t}, \mathrm{C}_{1^{\prime \prime}}\right), 25.7(\mathrm{t}$, $\mathrm{CH}_{2}$ from cyclohexane), 26.0 ( $\mathrm{t}, \mathrm{CH}_{2}$ from cyclohexane), $36.4(\mathrm{t}$, $\mathrm{CH}_{2}$ from cyclohexane), $44.4\left(\mathrm{t}, \mathrm{NCH}_{2} \mathrm{CH}_{2} \mathrm{OH}\right), 50.0\left(\mathrm{~d}, \mathrm{C}_{4 \mathrm{a}}\right), 51.6$ $\left(\mathrm{d}, \mathrm{C}_{8}\right), 61.2\left(\mathrm{t}, \mathrm{NCH}_{2} \mathrm{CH}_{2} \mathrm{OH}\right), 63.4\left(\mathrm{~d}, \mathrm{C}_{5}\right), 76.8\left(\mathrm{~s}, \mathrm{C}_{7}\right), 78.3(\mathrm{~d}$, $\left.\mathrm{C}_{8 \mathrm{a}}\right), 152.7\left(\mathrm{~s}, \mathrm{C}_{2}\right), 169.7\left(\mathrm{~s}, \mathrm{C}_{4}\right)$; HRMS calcd for $\mathrm{C}_{17} \mathrm{H}_{27} \mathrm{NO}_{5} \mathrm{Na}$ $\left[(\mathrm{M}+\mathrm{Na})^{+}\right] 348.1787$, found 348.1792 .

$\left(4 a S^{*}, 5 S^{*}, 7 S^{*}, 8 S^{*}, 8 a S^{*}\right)-8-$ Ethyl-3-(2-hydroxyethyl)-7-(2-methoxyphenyl)-5-methyltetrahydro- $2 \mathrm{H}, 5 \mathrm{H}$-pyrano $[3,4-e][1,3]-$ oxazine-2,4(3H)-dione (5r). Aldol $2 \mathbf{b}(54 \mathrm{mg}, 0.24 \mathrm{mmol})$ was subjected to the general procedure for the Prins cyclization and yielded, after purification by flash chromatography $(25 \mathrm{~cm}$ of height of silica gel, $n$-hexane/EtOAc 70:30), THF $8 \mathbf{r}(16 \mathrm{mg}, 20 \%, 80: 20 \mathrm{dr})$, title compound $5 \mathrm{r}(17 \mathrm{mg}, 20 \%,>95: 5 \mathrm{dr})$, and previously described bicycle $\mathbf{5 b}$ ( $3 \mathrm{mg}, 10 \%,>95: 5 \mathrm{dr})$. $\mathbf{5 r}$ was isolated as a thick colorless oil, and its description is given here: $R_{f}=0.28$ ( $n$-hexane/EtOAc 60:40, two times); ${ }^{1} \mathrm{H}$ NMR ( $\left.500 \mathrm{MHz}, \mathrm{CDCl}_{3}\right) \delta 0.73(\mathrm{t}, J=7.7 \mathrm{~Hz}$, $\left.3 \mathrm{H}, \mathrm{H}_{2^{\prime \prime \prime}}\right), 1.28-1.34\left(\mathrm{~m}, 1 \mathrm{H}, \mathrm{H}_{1^{\prime \prime}}\right), 1.47-1.53\left(\mathrm{~m}, 1 \mathrm{H}, \mathrm{H}_{1^{\prime \prime}}\right), 1.58$ $\left(\mathrm{d}, J=6.1 \mathrm{~Hz}, 3 \mathrm{H}, \mathrm{H}_{1^{\prime}}\right), 1.89-2.14\left(\mathrm{~m}, 2 \mathrm{H}, \mathrm{H}_{8}, \mathrm{OH}\right), 2.56(\mathrm{dd}, J=$ $\left.12.1,9.6 \mathrm{~Hz}, 1 \mathrm{H}, \mathrm{H}_{4 \mathrm{a}}\right), 3.77-3.86\left(\mathrm{~m}, 3 \mathrm{H}, \mathrm{H}_{5}, \mathrm{NCH}_{2} \mathrm{C}_{2} \mathrm{OH}\right), 3.81$ (s, $3 \mathrm{H}, \mathrm{MeO}$ ), 3.93 (ddd, $J=14.0,6.8,4.3 \mathrm{~Hz}, 1 \mathrm{H}, \mathrm{NC}_{2} \underline{\mathrm{CH}}_{2} \mathrm{OH}$ ), 4.13 (ddd, $J=14.0,5.8,4.3 \mathrm{~Hz}, 1 \mathrm{H}, \mathrm{NC}_{2} \mathrm{CH}_{2} \mathrm{OH}$ ), 4.39 (dd, $J=$ 11.6, $10.9 \mathrm{~Hz}, 1 \mathrm{H}, \mathrm{H}_{8 \mathrm{a}}$ ), 4.79 (br s, $\left.1 \mathrm{H}, \mathrm{H}_{7}\right), 6.88-6.89(\mathrm{~m}, 1 \mathrm{H}, \mathrm{Ar})$, 6.99-7.02 (m, 1H, Ar), 7.27-7.30 (m, 1H, Ar), 7.37-7.41 (m, $1 \mathrm{H}$, $\mathrm{Ar}) ;{ }^{13} \mathrm{C}$ NMR (150 MHz, $\left.\mathrm{CDCl}_{3}\right) \delta 10.3\left(\mathrm{q}, \mathrm{C}_{2^{\prime \prime \prime}}\right), 19.3\left(\mathrm{t}, \mathrm{C}_{1^{\prime \prime \prime}}\right)$, $21.1\left(\mathrm{q}, \mathrm{C}_{1^{\prime}}\right), 44.5\left(\mathrm{t}, \mathrm{NCH}_{2} \mathrm{CH}_{2} \mathrm{OH}\right), 47.1\left(\mathrm{~d}, \mathrm{C}_{8}\right), 49.2\left(\mathrm{~d}, \mathrm{C}_{4 \mathrm{a}}\right)$, $55.6(\mathrm{q}, \mathrm{MeO}), 61.1\left(\mathrm{t}, \mathrm{NCH}_{2} \mathrm{CH}_{2} \mathrm{OH}\right), 71.9\left(\mathrm{~d}, \mathrm{C}_{5}\right), 72.7\left(\mathrm{~d}, \mathrm{C}_{7}\right)$, 77.8 (d, $\mathrm{C}_{8 \mathrm{a}}$ ), 110.9 (d, $\left.\mathrm{C}_{3^{\prime \prime}}\right), 121.3\left(\mathrm{~d}, \mathrm{C}_{5^{\prime \prime}}\right), 127.6\left(\mathrm{~s}, \mathrm{C}_{1^{\prime \prime}}\right), 128.1(\mathrm{~d}$, $\left.\mathrm{C}_{6^{\prime \prime}}\right), 129.5\left(\mathrm{~d}, \mathrm{C}_{4^{\prime \prime}}\right), 152.4\left(\mathrm{~s}, \mathrm{C}_{2}\right), 156.8\left(\mathrm{~s}, \mathrm{C}_{2^{\prime \prime}}\right), 169.4\left(\mathrm{~s}, \mathrm{C}_{4}\right)$; HRMS calcd for $\mathrm{C}_{19} \mathrm{H}_{25} \mathrm{NO}_{6} \mathrm{Na}\left[(\mathrm{M}+\mathrm{Na})^{+}\right] 386.1580$, found 386.1589 .

$\left(4 a R^{*}, 5 R *, 7 R *, 8 R *, 8 a S^{*}\right)-5$-(4-Bromophenyl)-8-ethyl-3-(2-hydroxyethyl)-7-methyltetrahydro- $2 \mathrm{H}, 5 \mathrm{H}$-pyrano[3,4-e][1,3]oxazine2,4(3H)-dione (5u). Aldol $2 \mathrm{~h}(72 \mathrm{mg}, 0.20 \mathrm{mmol})$ and acetaldehyde $(0.09 \mathrm{~mL}$ of a $3.3 \mathrm{M}$ solution in DCM, $0.30 \mathrm{mmol}, 1.5$ equiv) were subjected to the general procedure for the synthesis of bicycles 5 (two-step EAP) and yielded, after purification by flash chromatography $(25 \mathrm{~cm}$ of height of silica gel, $n$-hexane/EtOAc 70:30), compound $5 \mathbf{u}(36 \mathrm{mg}, 43 \%,>95: 5 \mathrm{dr})$ and previously described bicycle $\mathbf{5 b}(3 \mathrm{mg}, 6 \%,>95: 5 \mathrm{dr})$. Alternatively, $\mathrm{N}$-acyl oxazolidin-2one $3 \mathrm{a}(320 \mathrm{mg}, 1.75 \mathrm{mmol})$ was subjected to the general procedure for the synthesis of bicycles 5 (one-pot EAP) and yielded, after purification by flash chromatography $(25 \mathrm{~cm}$ of height of silica gel, $n$ hexane/EtOAc 70:30), title compound $5 \mathbf{u}(155 \mathrm{mg}, 22 \%,>95: 5 \mathrm{dr}$ ) and a small amount of $\mathbf{5 b}(19 \mathrm{mg}, 5 \%,>95: 5 \mathrm{dr})$. $5 \mathbf{u}$ was isolated as a white solid, and its description is given here: $R_{f}=0.36$ ( $n$-hexane/ EtOAc 60:40, two times); mp $72-77{ }^{\circ} \mathrm{C}$ (from DCM $/ n$-hexane); ${ }^{1} \mathrm{H}$ $\operatorname{NMR}\left(600 \mathrm{MHz}, \mathrm{CDCl}_{3}\right) \delta 0.98\left(\mathrm{t}, J=7.5 \mathrm{~Hz}, 3 \mathrm{H}, \mathrm{H}_{2^{\prime \prime \prime}}\right), 1.31(\mathrm{~d}, J=$ $\left.6.2 \mathrm{~Hz}, 3 \mathrm{H}, \mathrm{H}_{1^{\prime \prime}}\right), 1.63-1.70\left(\mathrm{~m}, 1 \mathrm{H}, \mathrm{H}_{1^{\prime \prime}}\right), 1.73-1.84\left(\mathrm{~m}, 2 \mathrm{H}, \mathrm{H}_{8}\right.$, $\left.\mathrm{H}_{1^{\prime \prime \prime}}\right), 1.89$ (br s, $\left.1 \mathrm{H}, \mathrm{OH}\right), 2.94$ (dd, $J=11.9,10.0 \mathrm{~Hz}, 1 \mathrm{H}, \mathrm{H}_{4 \mathrm{a}}$ ), 3.50 $\left(\mathrm{dq}, J=9.7,6.1 \mathrm{~Hz}, 1 \mathrm{H}, \mathrm{H}_{7}\right), 3.67-3.77\left(\mathrm{~m}, 2 \mathrm{H}, \mathrm{NCH}_{2} \mathrm{CH}_{2} \mathrm{OH}\right)$, $3.78-3.83\left(\mathrm{~m}, 1 \mathrm{H}, \quad \mathrm{NCH}_{2} \mathrm{CH}_{2} \mathrm{OH}\right), 3.94-4.00(\mathrm{~m}, 1 \mathrm{H}$, $\left.\mathrm{NC}_{2} \mathrm{CH}_{2} \mathrm{OH}\right), 4.38\left(\mathrm{dd}, J=11.2,11.2 \mathrm{~Hz}, 1 \mathrm{H}, \mathrm{H}_{8 \mathrm{a}}\right), 4.47(\mathrm{~d}, J=$ $\left.10.0 \mathrm{~Hz}, 1 \mathrm{H}, \mathrm{H}_{5}\right), 7.30\left(\mathrm{~d}, J=8.4 \mathrm{~Hz}, 2 \mathrm{H}, 2 \times \mathrm{H}_{2^{\prime}}\right), 7.50(\mathrm{~d}, J=8.4$ $\left.\mathrm{Hz}, 2 \mathrm{H}, 2 \times \mathrm{H}_{3^{\prime}}\right) ;{ }^{13} \mathrm{C} \mathrm{NMR}\left(150 \mathrm{MHz}, \mathrm{CDCl}_{3}\right) \delta 9.6\left(\mathrm{q}, \mathrm{C}_{2^{\prime \prime}}\right), 18.9$ $\left(\mathrm{t}, \mathrm{C}_{1^{\prime \prime}}\right), 19.1\left(\mathrm{q}, \mathrm{C}_{1^{\prime \prime}}\right), 44.4\left(\mathrm{t}, \mathrm{NCH}_{2} \mathrm{CH}_{2} \mathrm{OH}\right), 46.9\left(\mathrm{~d}, \mathrm{C}_{8}\right), 48.3(\mathrm{~d}$, $\left.\mathrm{C}_{4 \mathrm{a}}\right), 60.8\left(\mathrm{t}, \mathrm{NCH}_{2} \mathrm{CH}_{2} \mathrm{OH}\right), 74.9\left(\mathrm{~d}, \mathrm{C}_{7}\right), 76.4\left(\mathrm{~d}, \mathrm{C}_{8 \mathrm{a}}\right), 76.8(\mathrm{~d}$, $\left.\mathrm{C}_{5}\right), 122.7\left(\mathrm{~s}, \mathrm{C}_{4^{\prime}}\right), 129.7\left(\mathrm{~d}, 2 \mathrm{C}, 2 \times \mathrm{C}_{2^{\prime}}\right), 131.6\left(\mathrm{~d}, 2 \mathrm{C}, 2 \times \mathrm{C}_{3^{\prime}}\right)$, $138.5\left(\mathrm{~s}, \mathrm{C}_{1^{\prime}}\right), 152.0\left(\mathrm{~s}, \mathrm{C}_{2}\right), 168.4\left(\mathrm{~s}, \mathrm{C}_{4}\right)$; HRMS calcd for $\mathrm{C}_{18} \mathrm{H}_{22}{ }^{79} \mathrm{BrNO}_{5} \mathrm{Na}\left[\left({ }^{79} \mathrm{M}+\mathrm{Na}\right)^{+}\right] 434.0579$, found 434.0584 .

(4aS, 5S,7R,8R,8aS)-8-Ethyl-3-[(R)-1-hydroxy-3-phenylpropan-2yl]-5,7-dimethyltetrahydropyrano [3,4-e][1,3]oxazine-2,4(3H,7H)dione (5ag). Aldol $2 \mathrm{i}(26 \mathrm{mg}, 83 \mu \mathrm{mol})$ and acetaldehyde $(37 \mu \mathrm{L}$ of a $3.3 \mathrm{M}$ solution in DCM, $125 \mathrm{mmol}, 1.5$ equiv) were subjected to the general procedure for the synthesis of bicycles 5 (two-step EAP) and yielded, after purification by flash chromatography $(18 \mathrm{~cm}$ of height of silica gel, $n$-hexane/EtOAc 70:30), 3-( $N$-acyl-oxazolidin-2-one)-THP 21a (3 mg, 9\%, >95:5 dr) and title bicycle 5 ag $(19 \mathrm{mg}, 62 \%,>95: 5$ dr). 5ag: yellowish oil; $R_{f}=0.31$ ( $n$-hexane/EtOAc 60:40); $[\alpha]^{25}$ -54.5 (c 1.0, $\left.\mathrm{CHCl}_{3}\right) ;{ }^{1} \mathrm{H}$ NMR $\left(600 \mathrm{MHz}, \mathrm{CDCl}_{3}\right) \delta 0.80(\mathrm{t}, J=7.5$ $\left.\mathrm{Hz}, 3 \mathrm{H}, \mathrm{H}_{2^{\prime \prime}}\right), 1.19\left(\mathrm{~d}, J=6.2 \mathrm{~Hz}, 3 \mathrm{H}, \mathrm{H}_{1^{\prime \prime}}\right), 1.40-1.47\left(\mathrm{~m}, 2 \mathrm{H}, \mathrm{H}_{8}\right.$, $\left.\mathrm{H}_{1^{\prime \prime \prime}}\right), 1.44\left(\mathrm{~d}, J=6.0 \mathrm{~Hz}, 3 \mathrm{H}, \mathrm{H}_{1^{\prime}}\right), 1.58-1.62\left(\mathrm{~m}, 1 \mathrm{H}, \mathrm{H}_{1^{\prime \prime}}\right), 2.14$ $\left(\mathrm{dd}, J=12.1,9.2 \mathrm{~Hz}, 1 \mathrm{H}, \mathrm{H}_{4 \mathrm{a}}\right), 2.81(\mathrm{br} \mathrm{s}, 1 \mathrm{H}, \mathrm{OH}), 3.05(\mathrm{dd}, J=$ 13.8, $\left.6.0 \mathrm{~Hz}, 1 \mathrm{H}, 1 \times \mathrm{CH}_{2} \mathrm{Ph}\right), 3.08\left(\right.$ br s, $\left.1 \mathrm{H}, \mathrm{H}_{7}\right), 3.13($ br s, $1 \mathrm{H}$, $\left.\mathrm{H}_{8 \mathrm{a}}\right), 3.19\left(\mathrm{dd}, J=14.0,11.4 \mathrm{~Hz}, 1 \mathrm{H}, 1 \times \mathrm{CH}_{2} \mathrm{Ph}\right), 3.26($ br s, $1 \mathrm{H}$, $\mathrm{H}_{5}$ ), 3.91 (dd, $\left.J=11.7,3.5 \mathrm{~Hz}, 1 \mathrm{H}, \mathrm{CH}_{2} \mathrm{OH}\right), 4.06-4.12($ br m, $1 \mathrm{H}$, $\left.\mathrm{CH}_{2} \mathrm{OH}\right), 5.13-5.20$ (br m, $\left.1 \mathrm{H}, \mathrm{NC} \underline{\mathrm{H}}\left(\mathrm{CH}_{2} \mathrm{Ph}\right) \mathrm{CH}_{2} \mathrm{OH}\right), 7.16-7.19$ $(\mathrm{m}, 2 \mathrm{H}, \mathrm{Ph}), 7.19-7.22(\mathrm{~m}, 1 \mathrm{H}, \mathrm{Ph}), 7.25-7.29(\mathrm{~m}, 2 \mathrm{H}, \mathrm{Ph}) ;{ }^{1} \mathrm{H}$ NMR $\left(600 \mathrm{MHz}, \mathrm{C}_{6} \mathrm{D}_{6}, 320 \mathrm{~K}\right) \delta 0.64\left(\mathrm{t}, J=7.6 \mathrm{~Hz}, 3 \mathrm{H}, \mathrm{H}_{2^{\prime \prime \prime}}\right), 0.96$ (d, $\left.J=6.2 \mathrm{~Hz}, 3 \mathrm{H}, \mathrm{H}_{1^{\prime \prime}}\right), 1.09-1.19\left(\mathrm{~m}, 2 \mathrm{H}, \mathrm{H}_{8}, \mathrm{H}_{1^{\prime \prime}}\right), 1.31-1.41(\mathrm{~m}$, $\left.1 \mathrm{H}, \mathrm{H}_{1^{\prime \prime}}\right), 1.49\left(\mathrm{~d}, J=6.0 \mathrm{~Hz}, 3 \mathrm{H}, \mathrm{H}_{1^{\prime}}\right), 1.65(\mathrm{dd}, J=12.0,9.6 \mathrm{~Hz}$, $\left.1 \mathrm{H}, \mathrm{H}_{4 \mathrm{a}}\right), 2.18(\mathrm{br} \mathrm{s}, 1 \mathrm{H}, \mathrm{OH}), 2.65\left(\mathrm{dq}, J=9.8,6.2 \mathrm{~Hz}, 1 \mathrm{H}, \mathrm{H}_{7}\right)$, $2.84\left(\mathrm{dd}, J=13.9,6.1 \mathrm{~Hz}, 1 \mathrm{H}, 1 \times \mathrm{C}_{2} \mathrm{Ph}\right), 3.03-3.10\left(\mathrm{~m}, 2 \mathrm{H}, \mathrm{H}_{5}\right.$, $\left.\mathrm{H}_{8 \mathrm{a}}\right), 3.21\left(\mathrm{dd}, J=14.0,11.2 \mathrm{~Hz}, 1 \mathrm{H}, 1 \times \mathrm{C}_{2} \mathrm{Ph}\right), 3.71(\mathrm{dd}, J=11.4$, $\left.4.4 \mathrm{~Hz}, 1 \mathrm{H}, \mathrm{CH}_{2} \mathrm{OH}\right), 4.06$ (dd, $J=11.4,7.4 \mathrm{~Hz}, 1 \mathrm{H}, \mathrm{CH}_{2} \mathrm{OH}$ ), 5.12-5.16 (br m, $\left.1 \mathrm{H}, \mathrm{NCH}\left(\mathrm{CH}_{2} \mathrm{Ph}\right) \mathrm{CH}_{2} \mathrm{OH}\right), 6.97-7.00(\mathrm{~m}, 1 \mathrm{H}$, $\mathrm{Ph})$, 7.06-7.09 (m, 1H, Ph), 7.11-7.13 (m, 2H, Ph); ${ }^{13} \mathrm{C}$ NMR (150 $\left.\mathrm{MHz}, \mathrm{CDCl}_{3}\right) \delta 9.2\left(\mathrm{q}, \mathrm{C}_{2^{\prime \prime \prime}}\right), 18.7\left(\mathrm{t}, \mathrm{C}_{1^{\prime \prime \prime}}\right), 18.9\left(\mathrm{q}, \mathrm{C}_{1^{\prime \prime}}\right), 20.9(\mathrm{q}$, $\left.\mathrm{C}_{1^{\prime}}\right), 33.3\left(\mathrm{t}, \mathrm{CH}_{2} \mathrm{Ph}\right), 46.4\left(\mathrm{~d}, \mathrm{C}_{8}\right), 48.8\left(\mathrm{~d}, \mathrm{C}_{4 \mathrm{a}}\right), 55.5(\mathrm{~d}$, $\left.\mathrm{NCH}\left(\mathrm{CH}_{2} \mathrm{Ph}\right) \mathrm{CH}_{2} \mathrm{OH}\right),{ }^{77} 63.5\left(\mathrm{t}, \underline{\mathrm{CH}}_{2} \mathrm{OH}\right), 71.0\left(\mathrm{~d}, \mathrm{C}_{5}\right), 73.6(\mathrm{~d}$, $\left.\mathrm{C}_{7}\right), 76.2\left(\mathrm{~d}, \mathrm{C}_{8 \mathrm{a}}\right){ }^{78} 126.9$ (d, Ph), 128.7 (d, 2C, Ph), 129.2 (d, 2C, $\mathrm{Ph}), 137.5$ (s, Ph), $151.9\left(\mathrm{~s}, \mathrm{C}_{2}\right), 169.4\left(\mathrm{~s}, \mathrm{C}_{4}\right) ;{ }^{13} \mathrm{C}$ NMR (150 MHz, $\left.\mathrm{C}_{6} \mathrm{D}_{6}, 320 \mathrm{~K}\right) \delta 9.7\left(\mathrm{q}, \mathrm{C}_{2^{\prime \prime \prime}}\right), 19.0\left(\mathrm{q}, \mathrm{C}_{1^{\prime \prime}}\right), 19.2\left(\mathrm{t}, \mathrm{C}_{1^{\prime \prime}}\right), 21.0(\mathrm{q}$, $\left.\mathrm{C}_{1^{\prime}}\right), 33.9\left(\mathrm{t}, \underline{\mathrm{CH}}_{2} \mathrm{Ph}\right), 46.9\left(\mathrm{~d}, \mathrm{C}_{8}\right), 49.0\left(\mathrm{~d}, \mathrm{C}_{4 \mathrm{a}}\right), 56.4(\mathrm{~d}$, $\left.\mathrm{NCH}\left(\mathrm{CH}_{2} \mathrm{Ph}\right) \mathrm{CH}_{2} \mathrm{OH}\right){ }^{79} 63.8\left(\mathrm{t}, \mathrm{CH}_{2} \mathrm{OH}\right), 71.1\left(\mathrm{~d}, \mathrm{C}_{5}\right), 73.6(\mathrm{~d}$, $\mathrm{C}_{7}$ ), $76.4\left(\mathrm{~d}, \mathrm{C}_{8 \mathrm{a}}\right.$ ), 126.8 (d, Ph), 128.7 (d, 2C, Ph), 129.6 (d, 2C, $\mathrm{Ph}), 138.4(\mathrm{~s}, \mathrm{Ph}), 151.5\left(\mathrm{~s}, \mathrm{C}_{2}\right), 169.5\left(\mathrm{~s}, \mathrm{C}_{4}\right) ; \mathrm{MS}(\mathrm{EI}) \mathrm{m} / \mathrm{z}$ (relative intensity) $361(\mathrm{M})^{+}(1), 343\left(\mathrm{M}-\mathrm{H}_{2} \mathrm{O}\right)^{+}(2), 228(\mathrm{M}+2-$ $\left.\mathrm{CH}(\mathrm{Bn}) \mathrm{CH}_{2} \mathrm{OH}\right)^{+}(83), 185(10), 184(\mathrm{M}-\mathrm{N}(\mathrm{CO}) \mathrm{CH}(\mathrm{Bn})-$ $\left.\mathrm{CH}_{2} \mathrm{OH}\right)^{+}$(78), 91 (100); HRMS calcd for $\mathrm{C}_{20} \mathrm{H}_{27} \mathrm{NO}_{5}\left[(\mathrm{M})^{+}\right]$ 361.1889, found 361.1884; HRMS calcd for $\mathrm{C}_{20} \mathrm{H}_{27} \mathrm{NO}_{5} \mathrm{Na}[(\mathrm{M}+$ $\left.\mathrm{Na})^{+}\right]$384.1787, found 384.1785 .

(4aR,5R,7S,8S,8aR)-8-Ethyl-3-[(S)-1-hydroxy-3-methylbutan-2yl]-5,7-dimethyltetrahydro-2H,5H-pyrano $[3,4-e][1,3]$ oxazine2,4(3H)-dione (5ah). Aldol $2 \mathrm{k}(58 \mathrm{mg}, 0.22 \mathrm{mmol}$ ) and acetaldehyde $(0.1 \mathrm{~mL}$ of a $3.3 \mathrm{M}$ solution in DCM, $0.33 \mathrm{mmol}, 1.5$ equiv) were subjected to the general procedure for the synthesis of bicycles 5 (two-step EAP) and yielded, after purification by flash chromatography (28 cm of height of silica gel, $n$-hexane/EtOAc 85:15), 3-( $N$ acyl-oxazolidin-2-one)-THP $21 \mathrm{~b}(11 \mathrm{mg}, 16 \%,>95: 5 \mathrm{dr})$ and title bicycle 5 ah $(29 \mathrm{mg}, 43 \%, 92: 8 \mathrm{dr})$. 5ah: colorless oil; $R_{f}=0.33(n-$ hexane/EtOAc 60:40); $[\alpha]^{25}{ }_{\mathrm{D}}+104.4\left(c 1.0, \mathrm{CHCl}_{3}\right) ;{ }^{1} \mathrm{H}$ NMR (500 $\left.\mathrm{MHz}, \mathrm{CDCl}_{3}\right) \delta 0.84\left(\mathrm{~d}, J=6.6 \mathrm{~Hz}, 3 \mathrm{H}, 1 \times\left(\mathrm{CH}_{3}\right)_{2} \mathrm{CH}\right), 0.92(\mathrm{t}, J=$ $\left.7.6 \mathrm{~Hz}, 3 \mathrm{H}, \mathrm{H}_{2^{\prime \prime \prime}}\right), 1.04\left(\mathrm{~d}, J=6.4 \mathrm{~Hz}, 3 \mathrm{H}, 1 \times\left(\mathrm{CH}_{3}\right)_{2} \mathrm{CH}\right), 1.27(\mathrm{~d}, J$ $\left.=6.1 \mathrm{~Hz}, 3 \mathrm{H}, \mathrm{H}_{1^{\prime \prime}}\right), 1.52\left(\mathrm{~d}, J=6.1 \mathrm{~Hz}, 3 \mathrm{H}, \mathrm{H}_{1^{\prime}}\right), 1.55-1.63(\mathrm{~m}, 2 \mathrm{H}$, $\left.\mathrm{H}_{8}, 1 \times \mathrm{H}_{1^{\prime \prime}}\right), 1.69-1.76\left(\mathrm{~m}, 1 \mathrm{H}, \mathrm{H}_{1^{\prime \prime}}\right), 2.36(\mathrm{dd}, J=12.2,9.8 \mathrm{~Hz}$, $\left.1 \mathrm{H}, \mathrm{H}_{4 \mathrm{a}}\right), 2.35-2.45\left(\mathrm{~m}, 1 \mathrm{H},\left(\mathrm{CH}_{3}\right)_{2} \mathrm{CH}\right), 2.89(\mathrm{br} \mathrm{s}, 1 \mathrm{H}, \mathrm{OH}), 3.31$ $\left(\mathrm{dq}, J=9.7,6.2 \mathrm{~Hz}, 1 \mathrm{H}, \mathrm{H}_{7}\right), 3.61\left(\mathrm{dq}, J=9.5,6.0 \mathrm{~Hz}, 1 \mathrm{H}, \mathrm{H}_{5}\right), 3.79$ (dd, $\left.J=12.1,2.7 \mathrm{~Hz}, 1 \mathrm{H}, 1 \times \mathrm{C}_{2} \mathrm{OH}\right), 4.01-4.08(\mathrm{~m}, 1 \mathrm{H}, 1 \times$ $\left.\mathrm{C}_{2} \mathrm{OH}\right), 4.11\left(\mathrm{dd}, J=12.1,10.4 \mathrm{~Hz}, 1 \mathrm{H}, \mathrm{H}_{8 \mathrm{a}}\right), 4.34-4.40(\mathrm{~m}, 1 \mathrm{H}$, $\left.\mathrm{NCH}\left(\mathrm{CH}_{2} \mathrm{OH}\right) \mathrm{CH}\left(\mathrm{CH}_{3}\right)_{2}\right) ;{ }^{13} \mathrm{C}$ NMR $\left(125 \mathrm{MHz}, \mathrm{CDCl}_{3}\right) \delta 9.7$ (q, $\left.\mathrm{C}_{2^{\prime \prime \prime}}\right), 19.0\left(\mathrm{t}, \mathrm{C}_{1^{\prime \prime \prime}}\right), 19.1\left(\mathrm{q}, \mathrm{C}_{1^{\prime \prime}}\right), 20.0\left(\mathrm{q}, 1 \times\left(\mathrm{CH}_{3}\right)_{2} \mathrm{CH}\right), 20.2(\mathrm{q}, 1$ $\left.\times\left(\underline{\mathrm{CH}}_{3}\right)_{2} \mathrm{CH}\right), 21.0\left(\mathrm{q}, \mathrm{C}_{1^{\prime}}\right), 25.4\left(\mathrm{~d},\left(\mathrm{CH}_{3}\right)_{2} \mathrm{CH}\right), 47.0\left(\mathrm{~d}, \mathrm{C}_{8}\right), 49.3$ $\left(\mathrm{d}, \mathrm{C}_{4 \mathrm{a}}\right), 62.3\left(\mathrm{~d}, \mathrm{NCH}\left(\mathrm{CH}_{2} \mathrm{OH}\right) \mathrm{CH}\left(\mathrm{CH}_{3}\right)_{2}\right),{ }^{80} 62.7\left(\mathrm{t}, \underline{\mathrm{CH}}_{2} \mathrm{OH}\right)$, $71.2\left(\mathrm{~d}, \mathrm{C}_{5}\right), 73.9\left(\mathrm{~d}, \mathrm{C}_{7}\right), 76.6\left(\mathrm{~d}, \mathrm{C}_{8 \mathrm{a}}\right), 152.3\left(\mathrm{~s}, \mathrm{C}_{2}\right), 169.8\left(\mathrm{~s}, \mathrm{C}_{4}\right)$; MS (EI) $m / z$ (relative intensity) $314(\mathrm{M}+\mathrm{H})^{+}(2), 284(\mathrm{M}-\mathrm{Et})^{+}$ or $(\mathrm{M}-2 \mathrm{Me})^{+}(11), 283(\mathrm{M}-\mathrm{Et}-\mathrm{H})^{+}$or $\left(\mathrm{M}+1-\mathrm{CH}_{2} \mathrm{OH}\right)^{+}$ $(66), 282\left(\mathrm{M}-\mathrm{CH}_{2} \mathrm{OH}\right)^{+}(18),{ }^{81} 240(\mathrm{M}+1-\mathrm{Et}-i-\mathrm{Pr})^{+}(2), 238$ 
$(\mathrm{M}-1-5 \mathrm{Me})^{+}(100), 228(\mathrm{M}+\mathrm{H}-\mathrm{Et}-\mathrm{Me}-i-\mathrm{Pr})^{+}$or $(\mathrm{M}+2$ $\left.-\mathrm{CH}(i-\mathrm{Pr}) \mathrm{CH}_{2} \mathrm{OH}\right)^{+}(94){ }^{81} 226\left(\mathrm{M}-\mathrm{CH}(i-\mathrm{Pr}) \mathrm{CH}_{2} \mathrm{OH}\right)^{+}(2)$, $184\left(\mathrm{M}+1-\mathrm{Et}-\mathrm{CH}(i-\mathrm{Pr}) \mathrm{CH}_{2} \mathrm{OH}-\mathrm{Me}\right)^{+}(92) ;{ }^{81} \mathrm{HRMS}$ calcd for $\mathrm{C}_{16} \mathrm{H}_{28} \mathrm{NO}_{5}\left[(\mathrm{M}+\mathrm{H})^{+}\right]$314.1967, found 314.1974.

(4aR,5R,7S,8S,8aR)-8-Ethyl-3-[(S)-1-hydroxy-3-methylbutan-2yl]-7-methyl-5-phenethyltetrahydro-2 $\mathrm{H}, 5 \mathrm{H}$-pyrano[3,4-e][1,3]oxazine-2,4(3H)-dione (5ai). Aldol 21 (43 mg, $0.12 \mathrm{mmol}$ ) and acetaldehyde $(0.05 \mathrm{~mL}$ of a $3.3 \mathrm{M}$ solution in DCM, $0.18 \mathrm{mmol}, 1.5$ equiv) were subjected to the general procedure for the synthesis of bicycles 5 (two-step EAP) and yielded, after purification by flash chromatography $(28 \mathrm{~cm}$ of height of silica gel, $n$-hexane/EtOAc 90:10), 3-( $N$-acyl-oxazolidin-2-one)-THP 21c (6 mg, 12\%, >95:5 dr) and title bicycle 5ai $(22 \mathrm{mg}, 45 \%,>95: 5 \mathrm{dr})$. 5ai: colorless oil; $R_{f}=$ 0.60 ( $n$-hexane/EtOAc 70:30, three times); $[\alpha]^{25}+97.7$ (c 0.9 , $\left.\mathrm{CHCl}_{3}\right) ;{ }^{1} \mathrm{H}$ NMR $\left(500 \mathrm{MHz}, \mathrm{CDCl}_{3}\right) \delta 0.82(\mathrm{~d}, J=6.7 \mathrm{~Hz}, 3 \mathrm{H}$, $\left.\left(\mathrm{CH}_{3}\right)_{2} \mathrm{CH}\right), 0.92\left(\mathrm{t}, J=7.5 \mathrm{~Hz}, 3 \mathrm{H}, \mathrm{H}_{2^{\prime \prime}}\right), 1.04(\mathrm{~d}, J=6.7 \mathrm{~Hz}, 3 \mathrm{H}$, $\left.\left(\underline{\mathrm{C}}_{3}\right)_{2} \mathrm{CH}\right), 1.32\left(\mathrm{~d}, J=6.1 \mathrm{~Hz}, 3 \mathrm{H}, \mathrm{H}_{1^{\prime \prime}}\right), 1.55-1.63\left(\mathrm{~m}, 2 \mathrm{H}, \mathrm{H}_{8}\right.$, $\left.\mathrm{H}_{1^{\prime \prime \prime}}\right), 1.68-1.76\left(\mathrm{~m}, 1 \mathrm{H}, \mathrm{H}_{1^{\prime \prime \prime}}\right), 1.81-1.88\left(\mathrm{~m}, 1 \mathrm{H}, \mathrm{H}_{1^{\prime}}\right), 2.35-2.45$ $\left(\mathrm{m},\left(\mathrm{CH}_{3}\right)_{2} \mathrm{CH}\right), 2.43\left(\mathrm{dd}, J=12.0,9.8 \mathrm{~Hz}, 1 \mathrm{H}, \mathrm{H}_{4 \mathrm{a}}\right), 2.59-2.66(\mathrm{~m}$, $\left.1 \mathrm{H}, \mathrm{H}_{1^{\prime}}\right), 2.71-2.77\left(\mathrm{~m}, 1 \mathrm{H}, \mathrm{H}_{2^{\prime}}\right), 2.85-2.91\left(\mathrm{~m}, 1 \mathrm{H}, \mathrm{H}_{2^{\prime}}\right), 3.25(\mathrm{dq}$, $\left.J=9.8,6.3 \mathrm{~Hz}, 1 \mathrm{H}, \mathrm{H}_{7}\right), 3.47\left(\mathrm{td}, J=9.5,2.2 \mathrm{~Hz}, 1 \mathrm{H}, \mathrm{H}_{5}\right), 3.78(\mathrm{dd}, J$ $\left.=12.2,2.8 \mathrm{~Hz}, 1 \mathrm{H}, \mathrm{CH}_{2} \mathrm{OH}\right), 4.03(\mathrm{dd}, J=12.2,7.5 \mathrm{~Hz}, 1 \mathrm{H}$, $\left.\mathrm{CH}_{2} \mathrm{OH}\right), 4.10\left(\mathrm{dd}, J=11.9,10.4 \mathrm{~Hz}, 1 \mathrm{H}, \mathrm{H}_{8 \mathrm{a}}\right), 4.32-4.38(\mathrm{~m}, 1 \mathrm{H}$, $\left.\mathrm{NC} \mathrm{H}(i-\mathrm{Pr}) \mathrm{CH}_{2} \mathrm{OH}\right), 7.16-7.20(\mathrm{~m}, 1 \mathrm{H}, \mathrm{Ph}), 7.21-7.24(\mathrm{~m}, 2 \mathrm{H}$, $\mathrm{Ph}), 7.26-7.30(\mathrm{~m}, 2 \mathrm{H}, \mathrm{Ph}) ;{ }^{13} \mathrm{C}$ NMR $\left(125 \mathrm{MHz}, \mathrm{CDCl}_{3}\right) \delta 9.7(\mathrm{q}$, $\left.\mathrm{C}_{2^{\prime \prime}}\right), 19.0\left(\mathrm{t}, \mathrm{C}_{1^{\prime \prime}}\right), 19.1\left(\mathrm{q}, \mathrm{C}_{1^{\prime \prime}}\right), 20.0\left(\mathrm{q},\left(\underline{\mathrm{C}}_{3}\right)_{2} \mathrm{CH}\right), 20.2(\mathrm{q}$, $\left.\left(\mathrm{CH}_{3}\right)_{2} \mathrm{CH}\right), 25.3\left(\mathrm{~d},\left(\mathrm{CH}_{3}\right)_{2} \underline{\mathrm{CH}}\right), 31.5\left(\mathrm{t}, \mathrm{C}_{2^{\prime}}\right), 35.7\left(\mathrm{t}, \mathrm{C}_{1^{\prime}}\right), 47.1(\mathrm{~d}$, $\left.\mathrm{C}_{8}\right), 47.6\left(\mathrm{~d}, \mathrm{C}_{4 \mathrm{a}}\right),{ }^{82} 62.7\left(\mathrm{t}, \underline{\mathrm{CH}}_{2} \mathrm{OH}\right), 73.7\left(\mathrm{~d}, \mathrm{C}_{5}\right), 73.9\left(\mathrm{~d}, \mathrm{C}_{7}\right), 76.7$ (d, $\mathrm{C}_{8 \mathrm{a}}$ ), 125.9 (d, Ph), 128.5 (d, 2C, Ph), 128.8 (d, 2C, Ph), 141.9 (s, $\mathrm{Ph}), 152.3\left(\mathrm{~s}, \mathrm{C}_{2}\right), 169.8\left(\mathrm{~s}, \mathrm{C}_{4}\right) ; \mathrm{MS}(\mathrm{EI}) \mathrm{m} / z$ (relative intensity) $403(\mathrm{M})^{+}(13), 385\left(\mathrm{M}-\mathrm{H}_{2} \mathrm{O}\right)^{+}(2){ }^{83} 283\left(\mathrm{M}-\mathrm{CH}_{2} \mathrm{OH}\right)^{+}(1)$, $359(\mathrm{M}+\mathrm{H}-i-\mathrm{Pr})^{+}(2), 316\left(\mathrm{M}-\mathrm{CH}(i-\mathrm{Pr}) \mathrm{CH}_{2} \mathrm{OH}\right)^{+}(4), 298(\mathrm{M}$ $\left.-\mathrm{CH}_{2} \mathrm{CH}_{2} \mathrm{Ph}\right)^{+}(22), 256\left(\mathrm{M}+\mathrm{H}-\mathrm{CH}_{2} \mathrm{CH}_{2} \mathrm{Ph}-i-\mathrm{Pr}\right)^{+}(18){ }^{83}$ HRMS calcd for $\mathrm{C}_{23} \mathrm{H}_{33} \mathrm{NO}_{5}\left[(\mathrm{M})^{+}\right]$403.2359, found 403.2385.

Synthesis of 2-Oxonia-Cope Rearranged Isomers 6. These byproducts could be punctually detected during the synthesis of bicycles $5.6 \mathrm{a}$ and $\mathbf{6 c}$ were described in our previous publication, ${ }^{30}$ and $6 \mathrm{~b}, 6 \mathrm{x}$, and $6 \mathrm{y}$ were never isolated.

Synthesis of Halogenated 2,3,4,5,6-Pentasubstituted THPs 1 and 7. (4aS*,5S*,7R*,8R*,8aS*)-3-(2-Chloroethyl)-8-ethyl-5,7diisobutyltetrahydro- $2 \mathrm{H}, 5 \mathrm{H}$-pyrano $[3,4-e][1,3]$ oxazine- $2,4(3 \mathrm{H})$ dione $(7 \mathrm{a}-\mathrm{Cl})$. To a suspension of bicycle $5 \mathrm{a}(86 \mathrm{mg}, 0.24 \mathrm{mmol})$ in $\mathrm{H}_{2} \mathrm{O}(2.4 \mathrm{~mL}, 0.1 \mathrm{M})$ was added a $37 \% \mathrm{HCl}$ aqueous solution $(2.4$ $\mathrm{mL}, 29 \mathrm{mmol}, 121$ equiv), and the mixture was heated at $100{ }^{\circ} \mathrm{C}$. After $4 \mathrm{~h}$, the mixture was allowed to cool to rt, saturated with $\mathrm{NaCl}$, and extracted with EtOAc $(5 \times 5 \mathrm{~mL})$. The combined organic layers were dried over $\mathrm{MgSO}_{4}$, filtered, concentrated, and purified by flash chromatography ( $21 \mathrm{~cm}$ of height of silica gel, $n$-hexane/EtOAc 93:7) to yield title compound $7 \mathrm{a}-\mathrm{Cl}(51 \mathrm{mg}, 57 \%)$ as a colorless oil: $R_{f}=$ 0.31 ( $n$-hexane/EtOAc 90:10); ${ }^{1} \mathrm{H}$ NMR $\left(500 \mathrm{MHz}, \mathrm{CDCl}_{3}\right) \delta 0.85-$ $0.94\left(\mathrm{~m}, 15 \mathrm{H}, 2 \times\left(\mathrm{C}_{3}\right)_{2} \mathrm{CHCH}_{2}, \mathrm{H}_{2^{\prime \prime \prime}}\right), 1.30-1.36(\mathrm{~m}, 1 \mathrm{H}$, $\left.\left(\mathrm{CH}_{3}\right)_{2} \mathrm{CHCH}_{2}\right), 1.41-1.52\left(\mathrm{~m}, 1 \mathrm{H},\left(\mathrm{CH}_{3}\right)_{2} \mathrm{CHCH}_{2}\right), 1.53-1.67$ $\left(\mathrm{m}, 2 \mathrm{H}, \mathrm{H}_{8}, \mathrm{H}_{1^{\prime \prime}}\right), 1.68-1.78\left(\mathrm{~m}, 1 \mathrm{H}, \mathrm{H}_{1^{\prime \prime}}\right), 1.85-1.97(\mathrm{~m}, 2 \mathrm{H}, 2 \times$ $\left.\left(\mathrm{CH}_{3}\right)_{2} \mathrm{CHCH}_{2}\right), 2.07-2.13\left(\mathrm{~m}, 1 \mathrm{H},\left(\mathrm{CH}_{3}\right)_{2} \mathrm{CHCH}_{2}\right), 2.38$ (dd, $J=$ $\left.12.0,9.6 \mathrm{~Hz}, 1 \mathrm{H}, \mathrm{H}_{4 \mathrm{a}}\right), 3.19\left(\mathrm{td}, J=10.3,2.1 \mathrm{~Hz}, 1 \mathrm{H}, \mathrm{H}_{7}\right), 3.53(\mathrm{td}, J$ $\left.=10.1,1.9 \mathrm{~Hz}, 1 \mathrm{H}, \mathrm{H}_{5}\right), 3.69\left(\mathrm{t}, J=6.3 \mathrm{~Hz}, \mathrm{NCH}_{2} \mathrm{CH}_{2} \mathrm{Cl}\right), 4.01-4.07$ $\left(\mathrm{m}, 1 \mathrm{H}, \mathrm{NCH}_{2} \mathrm{CH}_{2} \mathrm{Cl}\right.$ ), 4.13-4.18 (m, $1 \mathrm{H}, \mathrm{NCH}_{2} \mathrm{CH}_{2} \mathrm{Cl}$ ), 4.21 (dd, $J$ $\left.=11.7,10.4 \mathrm{~Hz}, 1 \mathrm{H}, \mathrm{H}_{8 \mathrm{a}}\right) ;{ }^{1} \mathrm{H} \operatorname{NMR}\left(500 \mathrm{MHz}, \mathrm{C}_{6} \mathrm{D}_{6}\right) \delta 0.69(\mathrm{t}, J=$ $\left.7.5 \mathrm{~Hz}, 3 \mathrm{H}, \mathrm{H}_{2^{\prime \prime}}\right), 0.81\left(\mathrm{~d}, J=6.6 \mathrm{~Hz}, 3 \mathrm{H},\left(\mathrm{CH}_{3}\right)_{2} \mathrm{CHCH}_{2}\right), 0.87(\mathrm{~d}, J$ $\left.=6.8 \mathrm{~Hz}, 3 \mathrm{H},\left(\mathrm{CH}_{3}\right)_{2} \mathrm{CHCH}_{2}\right), 0.98(\mathrm{~d}, J=6.7 \mathrm{~Hz}, 3 \mathrm{H}$, $\left.\left(\mathrm{CH}_{3}\right)_{2} \mathrm{CHCH}_{2}\right), 1.00\left(\mathrm{~d}, J=6.6 \mathrm{~Hz}, 3 \mathrm{H},\left(\mathrm{CH}_{3}\right)_{2} \mathrm{CHCH}_{2}\right), 1.09$ (ddd, $\left.J=13.5,10.5,2.3 \mathrm{~Hz}, 1 \mathrm{H}, \mathrm{H}_{1^{\prime \prime}}\right), 1.20-1.35\left(\mathrm{~m}, 3 \mathrm{H}, \mathrm{H}_{8}, \mathrm{H}_{1^{\prime \prime}}\right.$, $\left.\mathrm{H}_{1^{\prime \prime \prime}}\right), 1.36-1.54\left(\mathrm{~m}, 2 \mathrm{H}, \mathrm{H}_{1^{\prime}}, \mathrm{H}_{1^{\prime \prime \prime}}\right), 1.70(\mathrm{dd}, J=12.0,9.6 \mathrm{~Hz}, 1 \mathrm{H}$, $\left.\mathrm{H}_{4 \mathrm{a}}\right), 1.89-1.99\left(\mathrm{~m}, 1 \mathrm{H}, \mathrm{H}_{2^{\prime \prime}}\right), 2.00-2.09\left(\mathrm{~m}, 1 \mathrm{H}, \mathrm{H}_{2^{\prime}}\right), 2.22(\mathrm{ddd}, J=$ 13.6, 10.4, $\left.1.9 \mathrm{~Hz}, 1 \mathrm{H}, \mathrm{H}_{1^{\prime}}\right), 2.72\left(\mathrm{td}, J=10.5,1.5 \mathrm{~Hz}, 1 \mathrm{H}, \mathrm{H}_{7}\right), 3.20$ $\left(\mathrm{td}, J=9.8,1.9 \mathrm{~Hz}, 1 \mathrm{H}, \mathrm{H}_{5}\right), 3.37-3.48\left(\mathrm{~m}, 2 \mathrm{H}, \mathrm{NCH}_{2} \mathrm{CH}_{2} \mathrm{Cl}\right), 3.64$ $\left(\mathrm{dd}, J=11.9,10.4 \mathrm{~Hz}, 1 \mathrm{H}, \mathrm{H}_{8 \mathrm{a}}\right), 3.81(\mathrm{dt}, J=13.9,6.1 \mathrm{~Hz}, 1 \mathrm{H}$, $\left.\mathrm{NC}_{2} \mathrm{CH}_{2} \mathrm{Cl}\right), 3.97\left(\mathrm{dt}, J=13.9,6.7 \mathrm{~Hz}, 1 \mathrm{H}, \mathrm{NC}_{2} \mathrm{CH}_{2} \mathrm{Cl}\right) ;{ }^{13} \mathrm{C}$ NMR $\left(125 \mathrm{MHz}, \mathrm{CDCl}_{3}\right) \delta 9.3\left(\mathrm{q}, \mathrm{C}_{2^{\prime \prime \prime}}\right), 18.5\left(\mathrm{t}, \mathrm{C}_{1^{\prime \prime}}\right), 21.0(\mathrm{q}$, $\left.\left(\mathrm{C}_{3}\right)_{2} \mathrm{CHCH}_{2}\right), 21.1 \quad\left(\mathrm{q}, \quad\left(\underline{\mathrm{CH}}_{3}\right)_{2} \mathrm{CHCH}_{2}\right), 23.9$ (q,
$\left.\left(\underline{\mathrm{CH}}_{3}\right)_{2} \mathrm{CHCH}_{2}\right), 24.0 \quad\left(\mathrm{q}, \quad\left(\underline{\mathrm{CH}}_{3}\right)_{2} \mathrm{CHCH}_{2}\right), 24.1$ (d, $\left.\left.\left(\mathrm{CH}_{3}\right)_{2} \mathrm{CHCH}_{2}\right), 24.3\left(\mathrm{~d},\left(\mathrm{CH}_{3}\right)_{2} \underline{\mathrm{CHCH}}\right)_{2}\right), 40.7\left(\mathrm{t}, \mathrm{NCH}_{2} \mathrm{CH}_{2} \mathrm{Cl}\right)$, $41.5\left(\mathrm{t}, \quad\left(\mathrm{CH}_{3}\right)_{2} \mathrm{CHCH}{ }_{2}\right), 42.9\left(\mathrm{t}, \mathrm{NCH}_{2} \mathrm{CH}_{2} \mathrm{Cl}\right), 43.4(\mathrm{t}$, $\left.\left(\mathrm{CH}_{3}\right)_{2} \mathrm{CHCH}_{2}\right), 45.6\left(\mathrm{~d}, \mathrm{C}_{8}\right), 48.1\left(\mathrm{~d}, \mathrm{C}_{4 \mathrm{a}}\right), 72.8\left(\mathrm{~d}, \mathrm{C}_{5}\right), 75.1$ (d, $\left.\mathrm{C}_{7}\right), 76.8\left(\mathrm{~d}, \mathrm{C}_{8 \mathrm{a}}\right), 151.4\left(\mathrm{~s}, \mathrm{C}_{2}\right), 168.7\left(\mathrm{~s}, \mathrm{C}_{4}\right) ;{ }^{13} \mathrm{C}$ NMR $(125 \mathrm{MHz}$, $\left.\mathrm{C}_{6} \mathrm{D}_{6}\right) \delta 10.0\left(\mathrm{q}, \mathrm{C}_{2^{\prime \prime \prime}}\right), 19.1\left(\mathrm{t}, \mathrm{C}_{1^{\prime \prime}}\right), 21.3\left(\mathrm{q},\left(\mathrm{CH}_{3}\right)_{2} \mathrm{CHCH}_{2}\right), 21.6$ (q, $\left.\left(\mathrm{CH}_{3}\right)_{2} \mathrm{CHCH}_{2}\right), 24.0\left(\mathrm{q}, 2 \mathrm{C}, 2 \times\left(\mathrm{CH}_{3}\right)_{2} \mathrm{CHCH}_{2}\right), 24.4\left(\mathrm{~d}, \mathrm{C}_{2^{\prime \prime}}\right)$, $24.9\left(\mathrm{~d}, \mathrm{C}_{2^{\prime}}\right), 41.0\left(\mathrm{t}, \mathrm{NCH}_{2} \mathrm{CH}_{2} \mathrm{Cl}\right), 42.0\left(\mathrm{t}, \mathrm{C}_{1^{\prime \prime}}\right), 42.9(\mathrm{t}$, $\left.\mathrm{NCH}_{2} \mathrm{CH}_{2} \mathrm{Cl}\right), 43.9\left(\mathrm{t}, \mathrm{C}_{1^{\prime}}\right), 46.1\left(\mathrm{~d}, \mathrm{C}_{8}\right), 47.9\left(\mathrm{~d}, \mathrm{C}_{4 \mathrm{a}}\right), 72.9$ (d, $\mathrm{C}_{5}$ ), $75.3\left(\mathrm{~d}, \mathrm{C}_{7}\right), 76.9$ (d, $\mathrm{C}_{8 \mathrm{a}}$ ), $150.8\left(\mathrm{~s}, \mathrm{C}_{2}\right), 168.6\left(\mathrm{~s}, \mathrm{C}_{4}\right)$; MS (EI) $\mathrm{m} / z$ (relative intensity) $375\left({ }^{37} \mathrm{Cl}-\mathrm{M}\right)^{+}(1), 373\left({ }^{35} \mathrm{Cl}-\mathrm{M}\right)^{+}(3)$, $316(\mathrm{M}-i-\mathrm{Bu})^{+}(100), 287(\mathrm{M}-i-\mathrm{Bu}-\mathrm{Et})^{+}(9), 259(\mathrm{M}-2 i-$ $\mathrm{Bu})^{+}(17)$; HRMS calcd for $\mathrm{C}_{19} \mathrm{H}_{32} \mathrm{NO}_{4} \mathrm{Cl}\left[(\mathrm{M})^{+}\right]$373.2020, found 373.2014; HRMS calcd for $\mathrm{C}_{19} \mathrm{H}_{32} \mathrm{NO}_{4} \mathrm{Na}^{37} \mathrm{Cl}\left[(\mathrm{M}+\mathrm{Na})^{+}\right]$ 398.1888, found 398.1898.

(4aS, 5S,7R,8R,8aS)-3-(2-Bromoethyl)-8-ethyl-5,7-diisobutyltetrahydro-2H,5H-pyrano[3,4-e][1,3]oxazine-2,4(3H)-dione (7a-Br). To a suspension of bicycle $5 \mathrm{a}(1.55 \mathrm{~g}, 4.3 \mathrm{mmol})$ in $\mathrm{H}_{2} \mathrm{O}(4.3 \mathrm{~mL}, 0.1 \mathrm{M})$ was added a $48 \% \mathrm{HBr}$ aqueous solution $(58.5 \mathrm{~mL}, 520 \mathrm{mmol}, 121$ equiv), and the mixture was heated at $100{ }^{\circ} \mathrm{C}$. After $24 \mathrm{~h}$, the mixture was allowed to cool to rt, saturated with $\mathrm{NaCl}$, and extracted with DCM $(3 \times 15 \mathrm{~mL})$. The combined organic layers were dried over $\mathrm{MgSO}_{4}$, filtered, concentrated, and purified by flash chromatography ( $6 \mathrm{~cm}$ of height of silica gel, $n$-hexane/EtOAc $60: 40)$ to yield title compound $7 \mathbf{a}-\mathrm{Br}(1.45 \mathrm{~g}, 81 \%)$ as a thick brown oil: $R_{f}=0.3(n-$ hexane/EtOAc 60:40); ${ }^{1} \mathrm{H}$ NMR $\left(500 \mathrm{MHz}, \mathrm{CDCl}_{3}\right) \delta 0.84-0.88$ $\left(\mathrm{m}, 3 \mathrm{H}, \mathrm{H}_{2^{\prime \prime}}\right), 0.90-0.97\left(\mathrm{~m}, 12 \mathrm{H}, 4 \times\left(\mathrm{CH}_{3}\right)_{2} \mathrm{CH}\right), 1.30-1.37(\mathrm{~m}$, $\left.1 \mathrm{H}, \mathrm{H}_{1^{\prime \prime}}\right), 1.40-1.50\left(\mathrm{~m}, 2 \mathrm{H}, \mathrm{H}^{\prime}, \mathrm{H}_{1^{\prime \prime}}\right), 1.52-1.65\left(\mathrm{~m}, 2 \mathrm{H}, \mathrm{H}_{8}, \mathrm{H}_{1^{\prime \prime}}\right)$, $1.70-1.76\left(\mathrm{~m}, 1 \mathrm{H}, \mathrm{H}_{1^{\prime \prime}}\right), 1.85-1.96\left(\mathrm{~m}, 2 \mathrm{H}, \mathrm{H}_{2^{\prime}}, \mathrm{H}_{2^{\prime \prime}}\right), 2.06-2.14$ $\left(\mathrm{m}, 1 \mathrm{H}, \mathrm{H}_{1^{\prime}}\right), 2.34-2.40\left(\mathrm{~m}, 1 \mathrm{H}, \mathrm{H}_{4 \mathrm{a}}\right), 3.16-3.23\left(\mathrm{~m}, 1 \mathrm{H}, \mathrm{H}_{7}\right), 3.49-$ $3.56\left(\mathrm{~m}, 3 \mathrm{H}, \mathrm{H}_{5}, \mathrm{NCH}_{2} \mathrm{CH}_{2} \mathrm{Br}\right), 4.05-4.14\left(\mathrm{~m}, 1 \mathrm{H}, \mathrm{NCH}_{2} \mathrm{CH}_{2} \mathrm{Br}\right)$, 4.15-4.26 (m, 2H, $\left.\mathrm{H}_{8 a} \quad \mathrm{NCH}_{2} \mathrm{CH}_{2} \mathrm{Br}\right) ;{ }^{13} \mathrm{C} \mathrm{NMR}(125 \mathrm{MHz}$, $\left.\mathrm{CDCl}_{3}\right) \delta 9.3\left(\mathrm{q}, \mathrm{C}_{2^{\prime \prime \prime}}\right), 18.5\left(\mathrm{t}, \mathrm{C}_{1^{\prime \prime \prime}}\right), 21.0\left(\mathrm{q},\left(\mathrm{CH}_{3}\right)_{2} \mathrm{CHCH}_{2}\right), 21.2$ $\left(\mathrm{q}, \quad\left(\mathrm{CH}_{3}\right)_{2} \mathrm{CHCH}_{2}\right), 23.9\left(\mathrm{q},\left(\mathrm{CH}_{3}\right)_{2} \mathrm{CHCH}_{2}\right), 24.0$ (q, $\left.\left(\mathrm{CH}_{3}\right)_{2} \mathrm{CHCH}_{2}\right), 24.1$ (d, $\left.\left(\mathrm{CH}_{3}\right)_{2} \mathrm{CHCH}_{2}\right), 24.4$ (d, $\left.\left(\mathrm{CH}_{3}\right)_{2} \mathrm{CHCH}_{2}\right), 28.3\left(\mathrm{t}, \mathrm{NCH}_{2} \mathrm{CH}_{2} \mathrm{Br}\right), 41.6\left(\mathrm{t},\left(\mathrm{CH}_{3}\right)_{2} \mathrm{CHCH}_{2}\right)$, $42.8\left(\mathrm{t}, \mathrm{NCH}_{2} \mathrm{CH}_{2} \mathrm{Br}\right), 43.4\left(\mathrm{t},\left(\mathrm{CH}_{3}\right)_{2} \mathrm{CHCH}_{2}\right), 45.6\left(\mathrm{~d}, \mathrm{C}_{8}\right), 48.1$ $\left(\mathrm{d}, \mathrm{C}_{4 \mathrm{a}}\right), 72.9\left(\mathrm{~d}, \mathrm{C}_{5}\right), 75.2\left(\mathrm{~d}, \mathrm{C}_{8 \mathrm{a}}\right), 76.8\left(\mathrm{~d}, \mathrm{C}_{7}\right), 151.3\left(\mathrm{~s}, \mathrm{C}_{2}\right), 168.6$ (s, $\left.\mathrm{C}_{4}\right)$; HRMS calcd for $\mathrm{C}_{19} \mathrm{H}_{32} \mathrm{BrNO}_{4} \mathrm{Na}\left[(\mathrm{M}+\mathrm{Na})^{+}\right]$440.1392, found 440.1410 .

(4aS*,5S*,7R*,8R*,8aS*)-3-(2-Chloroethyl)-8-ethyl-5,7-dimethyltetrahydro-2H,5H-pyrano[3,4-e][1,3]oxazine-2,4(3H)-dione $(7 b-C l)$. To a solution of aldol $2 b(37 \mathrm{mg}, 0.16 \mathrm{mmol})$ in DCM $(1.6$ $\mathrm{mL}, 0.1 \mathrm{M})$ were sequentially added acetaldehyde $(73 \mu \mathrm{L}$ of a $3.3 \mathrm{M}$ solution in DCM, $0.24 \mathrm{mmol}, 1.5$ equiv), TMSCl $(0.05 \mathrm{~mL}, 0.40$ mmol, 2.5 equiv), and $\mathrm{BF}_{3} \cdot \mathrm{OEt}_{2}(0.01 \mathrm{~mL}, 0.08 \mathrm{mmol}, 0.5$ equiv; smaller amounts led to longer reaction times and worse yields of the bicycle). After $2 \mathrm{~h}$, the reaction was quenched by adding $\mathrm{H}_{2} \mathrm{O}(2 \mathrm{~mL})$, the layers were separated, and the aqueous layer was extracted with DCM $(3 \times 2 \mathrm{~mL})$. The combined organic layers were dried over $\mathrm{MgSO}_{4}$, filtered, concentrated, and purified by flash chromatography $(16 \mathrm{~cm}$ of height of silica gel, $n$-hexane/EtOAc $70: 30)$ to yield title compound $7 \mathbf{b}-\mathbf{C l}(31 \mathrm{mg}, 67 \%,>95: 5 \mathrm{dr})$ and rearranged byproduct 6b $(2 \mathrm{mg}, 6 \%) .7 \mathrm{~b}-\mathrm{Cl}$ was isolated as a white solid, and its description is given here: $R_{f}=0.60$ ( $n$-hexane/EtOAc $\left.60: 40\right) ; \mathrm{mp} 53-57{ }^{\circ} \mathrm{C}$ (from DCM $/ n$-hexane); ${ }^{1} \mathrm{H}$ NMR $\left(500 \mathrm{MHz}, \mathrm{CDCl}_{3}\right) \delta 0.93(\mathrm{t}, J=$ $\left.7.5 \mathrm{~Hz}, 3 \mathrm{H}, \mathrm{H}_{2^{\prime \prime}}\right), 1.28\left(\mathrm{~d}, J=6.1 \mathrm{~Hz}, 3 \mathrm{H}, \mathrm{H}_{1^{\prime \prime}}\right), 1.55(\mathrm{~d}, J=6.0 \mathrm{~Hz}$, $\left.3 \mathrm{H}, \mathrm{H}_{1^{\prime}}\right), 1.57-1.64\left(\mathrm{~m}, 2 \mathrm{H}, \mathrm{H}_{8}, \mathrm{H}_{1^{\prime \prime}}\right), 1.70-1.77\left(\mathrm{~m}, 1 \mathrm{H}, \mathrm{H}_{1^{\prime \prime}}\right), 2.38$ $\left(\mathrm{dd}, J=12.1,9.6 \mathrm{~Hz}, 1 \mathrm{H}, \mathrm{H}_{4 \mathrm{a}}\right), 3.33\left(\mathrm{dq}, J=9.8,6.1 \mathrm{~Hz}, 1 \mathrm{H}, \mathrm{H}_{7}\right)$, $3.62\left(\mathrm{dq}, J=9.6,6.0 \mathrm{~Hz}, 1 \mathrm{H}, \mathrm{H}_{5}\right), 3.71(\mathrm{t}, J=6.4 \mathrm{~Hz}, 2 \mathrm{H}$, $\left.\mathrm{NCH}_{2} \mathrm{CH}_{2} \mathrm{Cl}\right), 4.08\left(\mathrm{dt}, J=13.8,6.3 \mathrm{~Hz}, 1 \mathrm{H}, \mathrm{NCH}_{2} \mathrm{CH}_{2} \mathrm{Cl}\right), 4.17$ $\left(\mathrm{dt}, J=13.9,6.6 \mathrm{~Hz}, 1 \mathrm{H}, \mathrm{NCH}_{2} \mathrm{CH}_{2} \mathrm{Cl}\right), 4.19(\mathrm{dd}, J=12.2,10.3 \mathrm{~Hz}$, $\left.1 \mathrm{H}, \mathrm{H}_{8 \mathrm{a}}\right) ;{ }^{13} \mathrm{C} \operatorname{NMR}\left(125 \mathrm{MHz}, \mathrm{CDCl}_{3}\right) \delta 9.6\left(\mathrm{q}, \mathrm{C}_{2^{\prime \prime \prime}}\right), 18.9\left(\mathrm{t}, \mathrm{C}_{1^{\prime \prime \prime}}\right)$, $19.1\left(\mathrm{q}, \mathrm{C}_{1^{\prime \prime}}\right), 21.0\left(\mathrm{q}, \mathrm{C}_{1^{\prime}}\right), 40.7\left(\mathrm{t}, \mathrm{NCH}_{2} \mathrm{CH}_{2} \mathrm{Cl}\right), 42.9(\mathrm{t}$, $\left.\mathrm{NCH}_{2} \mathrm{CH}_{2} \mathrm{Cl}\right), 46.9$ (d, $\left.\mathrm{C}_{8}\right), 49.1\left(\mathrm{~d}, \mathrm{C}_{4 \mathrm{a}}\right), 71.1$ (d, $\left.\mathrm{C}_{5}\right), 73.9$ (d, $\left.\mathrm{C}_{7}\right), 76.5\left(\mathrm{~d}, \mathrm{C}_{8 \mathrm{a}}\right), 151.4\left(\mathrm{~s}, \mathrm{C}_{4}\right), 168.5\left(\mathrm{~s}, \mathrm{C}_{2}\right) ; \mathrm{MS}(\mathrm{EI}) \mathrm{m} / \mathrm{z}$ (relative intensity) $289(\mathrm{M})^{+}(4), 274(\mathrm{M}-\mathrm{Me})^{+}(16), 246(\mathrm{M}-\mathrm{Et}-\mathrm{Me})^{+}$ (3), $230(\mathrm{M}-1-\mathrm{Et}-2 \mathrm{Me})^{+}(4)$; HRMS calcd for $\mathrm{C}_{13} \mathrm{H}_{20} \mathrm{NO}_{4} \mathrm{Na}^{35} \mathrm{Cl}\left[(\mathrm{M}+\mathrm{Na})^{+}\right] 312.0979$, found 312.0970; HRMS calcd for $\mathrm{C}_{13} \mathrm{H}_{20} \mathrm{NO}_{4}{ }^{35} \mathrm{Cl}\left[(\mathrm{M})^{+}\right]$289.1081, found 289.1089. 
$(4 a S *, 5 S *, 7 R *, 8 R *, 8 a S *)-3-(2-B r o m o e t h y l)-8$-ethyl-5,7-dimethyltetrahydro-2H,5H-pyrano[3,4-e][1,3]oxazine-2,4(3H)-dione (7b-Br) and 3-[(2S*,3R*,4S*,5R*,6R*)-4-Bromo-5-ethyl-2,6-dimethyltetrahydro-2H-pyran-3-carbonyl]oxazolidin-2-one (1b-Br). To a solution of aldol $\mathbf{2 b}(50 \mathrm{mg}, 0.22 \mathrm{mmol})$ and acetaldehyde $(0.1 \mathrm{~mL}$ of a $3.3 \mathrm{M}$ solution in DCM, $0.33 \mathrm{mmol}, 1.5$ equiv) in DCM $(2.2 \mathrm{~mL}$, $0.1 \mathrm{M})$ was added TMSBr $(0.08 \mathrm{~mL}, 0.55 \mathrm{mmol}, 2.5$ equiv). After $3 \mathrm{~h}$, the reaction was stopped by the addition of $\mathrm{H}_{2} \mathrm{O}(3 \mathrm{~mL})$ and the aqueous layer was extracted with DCM $(3 \times 3 \mathrm{~mL})$. The combined organic layers were dried over $\mathrm{MgSO}_{4}$, filtered, and concentrated. ${ }^{1} \mathrm{H}$ NMR analysis of the crude revealed a 1.5:1 mixture of isomers $7 \mathbf{b}-\mathbf{B r}$ and $\mathbf{1 b}-\mathrm{Br}$. Purification by flash chromatography $(16 \mathrm{~cm}$ of height of silica gel, $n$-hexane/EtOAc 90:10) allowed their separation, yielding bicycle $7 \mathrm{~b}-\mathrm{Br}(34 \mathrm{mg}, 46 \%,>95: 5 \mathrm{dr})$ and 3-(N-acyl-oxazolidin-2one)-THP 1b-Br (22 mg, 30\%, >95:5 dr). $7 \mathbf{b}-\mathbf{B r}$ : yellowish oil; $R_{f}=$ 0.53 ( $n$-hexane/EtOAc 80:20); ${ }^{1} \mathrm{H}$ NMR $\left(500 \mathrm{MHz}, \mathrm{CDCl}_{3}\right) \delta 0.92$ $\left(\mathrm{t}, J=7.5 \mathrm{~Hz}, 3 \mathrm{H}, \mathrm{H}_{2^{\prime \prime \prime}}\right), 1.27\left(\mathrm{~d}, J=6.2 \mathrm{~Hz}, 3 \mathrm{H}, \mathrm{H}_{1^{\prime \prime}}\right), 1.54(\mathrm{~d}, J=6.0$ $\left.\mathrm{Hz}, 3 \mathrm{H}, \mathrm{H}_{1^{\prime}}\right), 1.56-1.63\left(\mathrm{~m}, 2 \mathrm{H}, \mathrm{H}_{8}, \mathrm{H}_{1^{\prime \prime \prime}}\right), 1.69-1.77\left(\mathrm{~m}, 1 \mathrm{H}, \mathrm{H}_{1^{\prime \prime \prime}}\right)$, $2.36\left(\mathrm{dd}, J=12.1,9.8 \mathrm{~Hz}, 1 \mathrm{H}, \mathrm{H}_{4 \mathrm{a}}\right), 3.32(\mathrm{dq}, J=9.8,6.1 \mathrm{~Hz}, 1 \mathrm{H}$, $\left.\mathrm{H}_{7}\right), 3.54\left(\mathrm{t}, J=6.7 \mathrm{~Hz}, 2 \mathrm{H}, \mathrm{NCH}_{2} \mathrm{C}_{2} \mathrm{Br}\right), 3.62(\mathrm{dq}, J=9.7,6.1 \mathrm{~Hz}$, $\left.1 \mathrm{H}, \mathrm{H}_{5}\right), 4.09-4.14\left(\mathrm{~m}, 1 \mathrm{H}, \mathrm{NCH}_{2} \mathrm{CH}_{2} \mathrm{Br}\right), 4.17-4.23\left(\mathrm{~m}, 2 \mathrm{H}, \mathrm{H}_{8 \mathrm{a}}, 1\right.$ $\left.\times \mathrm{NCH}_{2} \mathrm{CH}_{2} \mathrm{Br}\right) ;{ }^{13} \mathrm{C}$ NMR $\left(150 \mathrm{MHz}, \mathrm{CDCl}_{3}\right) \delta 9.5\left(\mathrm{q}, \mathrm{C}_{2^{\prime \prime}}\right), 18.9$ (t, $\left.\mathrm{C}_{1^{\prime \prime}}\right), 19.1$ (q, $\mathrm{C}_{1^{\prime \prime}}$ ), 20.9 (q, $\mathrm{C}_{1^{\prime}}$ ), $28.3\left(\mathrm{t}, \mathrm{NCH}_{2} \mathrm{CH}_{2} \mathrm{Br}\right.$ ), 42.7 (t, $\left.\mathrm{NCH}_{2} \mathrm{CH}_{2} \mathrm{Br}\right), 46.8\left(\mathrm{~d}, \mathrm{C}_{8}\right), 49.1\left(\mathrm{~d}, \mathrm{C}_{4 \mathrm{a}}\right), 71.0\left(\mathrm{~d}, \mathrm{C}_{5}\right), 73.9\left(\mathrm{~d}, \mathrm{C}_{7}\right)$, $76.5\left(\mathrm{~d}, \mathrm{C}_{8 \mathrm{a}}\right), 151.2\left(\mathrm{~s}, \mathrm{C}_{2}\right), 168.4\left(\mathrm{~s}, \mathrm{C}_{4}\right) ; \mathrm{MS}(\mathrm{EI}) \mathrm{m} / \mathrm{z}$ (relative intensity) $335\left({ }^{81} \mathrm{M}\right)^{+}(4), 333\left({ }^{79} \mathrm{M}\right)^{+}(3), 319\left({ }^{81} \mathrm{M}-\mathrm{Me}\right)^{+}(13)$, $317\left({ }^{79} \mathrm{M}-\mathrm{Me}\right)^{+}(13), 69$ (100); HRMS calcd for $\mathrm{C}_{13} \mathrm{H}_{20}{ }^{81} \mathrm{BrNO}_{4}$ $\left[\left({ }^{81} \mathrm{M}\right)^{+}\right]$335.0555, found 335.0544. 1b-Br: thick colorless oil; $R_{f}=$ 0.25 (n-hexane/EtOAc 80:20), 0.58 (n-hexane/EtOAc 60:40); ${ }^{1} \mathrm{H}$ $\operatorname{NMR}\left(500 \mathrm{MHz}, \mathrm{CDCl}_{3}\right) \delta 0.87\left(\mathrm{t}, J=7.6 \mathrm{~Hz}, 3 \mathrm{H}, \mathrm{C}_{5}, \mathrm{CH}_{2} \mathrm{CH}_{3}\right)$, $1.21\left(\mathrm{~d}, J=6.1 \mathrm{~Hz}, 3 \mathrm{H}, \mathrm{C}_{2^{\prime}} \underline{\mathrm{CH}}_{3}\right), 1.28\left(\mathrm{~d}, J=6.1 \mathrm{~Hz}, 3 \mathrm{H}, \mathrm{C}_{6} \underline{\mathrm{CH}}_{3}\right)$, $1.59-1.66\left(\mathrm{~m}, 1 \mathrm{H}, \mathrm{C}_{5^{\prime}} \mathrm{CH}_{2} \mathrm{CH}_{3}\right), 1.68-1.73\left(\mathrm{~m}, 1 \mathrm{H}, \mathrm{H}_{5^{\prime}}\right), 1.76-1.84$ $\left(\mathrm{m}, 1 \mathrm{H}, \mathrm{C}_{5^{\prime}} \underline{\mathrm{C}}_{2} \mathrm{CH}_{3}\right), 3.47\left(\mathrm{dq}, J=9.7,6.2 \mathrm{~Hz}, 1 \mathrm{H}, \mathrm{H}_{6^{\prime}}\right), 3.60(\mathrm{dq}, J$ $\left.=9.4,6.2 \mathrm{~Hz}, 1 \mathrm{H}, \mathrm{H}_{2^{\prime}}\right), 4.04\left(\mathrm{dt}, J=11.1,8.3 \mathrm{~Hz}, 1 \mathrm{H}, \mathrm{H}_{4}\right), 4.12(\mathrm{dt}, J$ $\left.=11.1,7.9 \mathrm{~Hz}, 1 \mathrm{H}, \mathrm{H}_{4}\right), 4.38\left(\mathrm{dd}, J=11.1,11.1 \mathrm{~Hz}, 1 \mathrm{H}, \mathrm{H}_{4^{\prime}}\right), 4.43(\mathrm{t}$, $\left.J=8.2 \mathrm{~Hz}, 2 \mathrm{H}, \mathrm{H}_{5}\right), 4.63\left(\mathrm{dd}, J=10.2,10.2 \mathrm{~Hz}, 1 \mathrm{H}, \mathrm{H}_{3^{\prime}}\right) ;{ }^{13} \mathrm{C} \mathrm{NMR}$ $\left(125 \mathrm{MHz}, \mathrm{CDCl}_{3}\right) \delta 8.8\left(\mathrm{q}, \mathrm{C}_{5^{\prime}} \mathrm{CH}_{2} \underline{\mathrm{CH}}_{3}\right), 21.9\left(\mathrm{t}, \mathrm{C}_{5^{\prime}} \underline{\mathrm{CH}}_{2} \mathrm{CH}_{3}\right)$, $19.6\left(\mathrm{q}, \mathrm{C}_{2^{\prime}} \underline{\mathrm{CH}_{3}} \mathrm{H}_{3}\right), 20.0\left(\mathrm{q}, \mathrm{C}_{6^{\prime}} \underline{\mathrm{CH}_{3}}\right), 42.9\left(\mathrm{t}, \mathrm{C}_{4}\right), 49.7\left(\mathrm{~d}, \mathrm{C}_{5^{\prime}}\right), 55.7$ $\left(\mathrm{d}, \mathrm{C}_{3^{\prime}}\right), 56.1\left(\mathrm{~d}, \mathrm{C}_{4^{\prime}}\right), 61.9\left(\mathrm{t}, \mathrm{C}_{5}\right), 76.1\left(\mathrm{~d}, \mathrm{C}_{2^{\prime}}\right), 76.2\left(\mathrm{~d}, \mathrm{C}_{6^{\prime}}\right), 153.1$ $\left(\mathrm{s}, \mathrm{C}_{2}\right), 172.5\left(\mathrm{~s}, \mathrm{C}_{3^{\prime}} \underline{\mathrm{C}}(\mathrm{O}) \mathrm{N}\right) ; \mathrm{MS}(\mathrm{EI}) \mathrm{m} / z$ (relative intensity) 254 $(\mathrm{M}-\mathrm{Br})^{+}(26), 210(\mathrm{M}-1-\mathrm{Br}-\mathrm{Et}-\mathrm{Me})^{+}(100), 168(\mathrm{M}-\mathrm{Br}$ - oxazolidin-2-one $)^{+}(3), 140(\mathrm{M}-\mathrm{Br}-\mathrm{N} \text {-acyl oxazolidin-2-one })^{+}$ (1); HRMS calcd for $\mathrm{C}_{13} \mathrm{H}_{20}{ }^{79} \mathrm{BrNO}_{4} \mathrm{Na}\left[\left({ }^{79} \mathrm{M}+\mathrm{Na}\right)^{+}\right] 356.0473$, found 356.0477 .

(4aS*,5S*,7R*,8R*,8aS*)-8-Ethyl-3-(2-iodoethyl)-5,7-dimethyltetrahydro-2H,5H-pyrano[3,4-e][1,3]oxazine-2,4(3H)-dione (7b-I) and $3-[(2 S *, 3 R *, 4 S *, 5 R *, 6 R *)-5$-Ethyl-4-iodo-2,6-dimethyltetrahydro-2H-pyran-3-carbonyl)oxazolidin-2-one (1b-l). To a solution of aldol $2 \mathbf{b}(50 \mathrm{mg}, 0.22 \mathrm{mmol})$ and acetaldehyde $(0.1 \mathrm{~mL}$ of a $3.3 \mathrm{M}$ solution in DCM, $0.33 \mathrm{mmol}, 1.5$ equiv) in DCM $(2.2 \mathrm{~mL}, 0.1 \mathrm{M})$ was added TMSI $(0.08 \mathrm{~mL}, 0.55 \mathrm{mmol}, 2.5$ equiv). TLC analysis showed that the reaction was completed at $12 \mathrm{~min}$, and $\mathrm{H}_{2} \mathrm{O}(3 \mathrm{~mL})$ was added. The aqueous layer was extracted with DCM $(3 \times 3 \mathrm{~mL})$, and the combined organic layers were dried over $\mathrm{MgSO}_{4}$, filtered, and concentrated. ${ }^{1} \mathrm{H}$ NMR analysis of the crude revealed a 3.4:1 mixture of isomers $\mathbf{7 b}$-I and $\mathbf{1 b}$-I. Purification by flash chromatography (16 $\mathrm{cm}$ of height of silica gel, $n$-hexane/EtOAc 90:10) allowed their separation, yielding bicycle $7 \mathbf{b}-\mathrm{I}(50 \mathrm{mg}, 58 \%,>95: 5 \mathrm{dr})$ and $3-(\mathrm{N}$ acyl oxazolidin-2-one)-THP $\mathbf{1 b}$-I $(14 \mathrm{mg}, 17 \%,>95: 5 \mathrm{dr})$. 7b-I: yellow oil; $R_{f}=0.22$ ( $n$-hexane/EtOAc 90:10), 0.63 ( $n$-hexane/EtOAc 60:40); ${ }^{1} \mathrm{H} \mathrm{NMR}\left(600 \mathrm{MHz}, \mathrm{CDCl}_{3}\right) \delta 0.94\left(\mathrm{t}, J=7.5 \mathrm{~Hz}, 3 \mathrm{H}, \mathrm{H}_{2^{\prime \prime \prime}}\right)$, $1.27\left(\mathrm{~d}, J=6.2 \mathrm{~Hz}, 3 \mathrm{H}, \mathrm{H}_{1^{\prime \prime}}\right), 1.54\left(\mathrm{~d}, J=6.0 \mathrm{~Hz}, 3 \mathrm{H}, \mathrm{H}_{1^{\prime}}\right), 1.56-1.65$ $\left(\mathrm{m}, 2 \mathrm{H}, \mathrm{H}_{8}, \mathrm{H}_{1^{\prime \prime}}\right), 1.69-1.76\left(\mathrm{~m}, 1 \mathrm{H}, \mathrm{H}_{1^{\prime \prime}}\right), 2.34$ (dd, $J=12.1,9.6$ $\left.\mathrm{Hz}, 1 \mathrm{H}, \mathrm{H}_{4 \mathrm{a}}\right), 3.29-3.37\left(\mathrm{~m}, 3 \mathrm{H}, \mathrm{H}_{7}, 2 \times \mathrm{NCH}_{2} \mathrm{CH}_{2} \mathrm{I}\right), 3.61(\mathrm{dq}, J=$ 9.7, $\left.6.0 \mathrm{~Hz}, 1 \mathrm{H}, \mathrm{H}_{5}\right), 4.03-4.08\left(\mathrm{~m}, 1 \mathrm{H}, \mathrm{NCH}_{2} \mathrm{CH}_{2} \mathrm{I}\right), 4.11-4.16(\mathrm{~m}$, $\left.1 \mathrm{H}, \mathrm{NCH}_{2} \mathrm{CH}_{2} \mathrm{I}\right), 4.20\left(\mathrm{dd}, J=11.8,10.7 \mathrm{~Hz}, 1 \mathrm{H}, \mathrm{H}_{8 \mathrm{a}}\right) ;{ }^{13} \mathrm{C} \mathrm{NMR}$ $\left(150 \mathrm{MHz}, \mathrm{CDCl}_{3}\right) \delta 0.3\left(\mathrm{t}, \mathrm{NCH}_{2} \mathrm{CH}_{2} \mathrm{I}\right), 9.7\left(\mathrm{q}, \mathrm{C}_{2^{\prime \prime}}\right), 19.11(\mathrm{t}$, $\left.\mathrm{C}_{1^{\prime \prime}}\right), 19.13\left(\mathrm{q}, \mathrm{C}_{1^{\prime \prime}}\right), 21.0\left(\mathrm{q}, \mathrm{C}_{1^{\prime}}\right), 43.5\left(\mathrm{t}, \mathrm{NCH}_{2} \mathrm{CH}_{2} \mathrm{I}\right), 47.1\left(\mathrm{~d}, \mathrm{C}_{8}\right)$, $49.4\left(\mathrm{~d}, \mathrm{C}_{4 \mathrm{a}}\right), 71.2\left(\mathrm{~d}, \mathrm{C}_{5}\right), 74.1\left(\mathrm{~d}, \mathrm{C}_{7}\right), 76.8\left(\mathrm{~d}, \mathrm{C}_{8 \mathrm{a}}\right), 151.1\left(\mathrm{~s}, \mathrm{C}_{2}\right)$, $168.3\left(\mathrm{~s}, \mathrm{C}_{4}\right) ; \mathrm{MS}(\mathrm{EI}) \mathrm{m} / \mathrm{z}$ (relative intensity) $267(3), 254(\mathrm{M}-\mathrm{I})^{+}$
(5), $228\left(\mathrm{M}+2-\mathrm{CH}_{2} \mathrm{CH}_{2} \mathrm{I}\right)^{+}(1), 210\left(\mathrm{M}-\mathrm{CH}_{2} \mathrm{CH}_{2} \mathrm{I}-\mathrm{Me}\right)^{+}$or $(\mathrm{M}-\mathrm{Et}-\mathrm{I}-\mathrm{Me})^{+}(56), 184\left(\mathrm{M}-\mathrm{CH}_{2} \mathrm{CH}_{2} \mathrm{I}-\mathrm{Et}-\mathrm{Me}\right)^{+}(1)$, 140, 91 (100); HRMS calcd for $\mathrm{C}_{13} \mathrm{H}_{20} \mathrm{NO}_{4}\left[(\mathrm{M}-\mathrm{I})^{+}\right]$254.1392, found 254.1384. 1b-I: yellow oil; $R_{f}=0.07$ ( $n$-hexane/EtOAc 90:10), 0.44 ( $n$-hexane/EtOAc 60:40); ${ }^{1} \mathrm{H}$ NMR (500 MHz, $\mathrm{CDCl}_{3}$ ) $\delta 0.83$ $\left(\mathrm{t}, J=7.5 \mathrm{~Hz}, 3 \mathrm{H}, \mathrm{C}_{5^{\prime}} \mathrm{CH}_{2} \mathrm{C}_{3}\right), 1.20\left(\mathrm{~d}, J=6.2 \mathrm{~Hz}, 3 \mathrm{H}, \mathrm{C}_{2}, \underline{\mathrm{H}}_{3}\right)$, $1.28\left(\mathrm{~d}, J=6.2 \mathrm{~Hz}, 3 \mathrm{H}, \mathrm{C}_{6} \mathrm{C}_{3}\right), 1.62-1.68(\mathrm{~m}, 1 \mathrm{H}, 1 \times$ $\mathrm{C}_{5^{\prime}} \mathrm{CH}_{2} \mathrm{CH}_{3}$ ), $1.70-1.79\left(\mathrm{~m}, 2 \mathrm{H}, \mathrm{H}_{5^{\prime}}, 1 \times \mathrm{C}_{5^{\prime}} \mathrm{CH}_{2} \mathrm{CH}_{3}\right), 3.49$ (dq, $J=$ 9.4, $\left.6.1 \mathrm{~Hz}, 1 \mathrm{H}, \mathrm{H}_{6^{\prime}}\right), 3.58\left(\mathrm{dq}, J=9.4,6.1 \mathrm{~Hz}, 1 \mathrm{H}, \mathrm{H}_{2^{\prime}}\right), 4.01-4.07$ $\left(\mathrm{m}, 1 \mathrm{H}, \mathrm{H}_{4}\right), 4.09-4.14\left(\mathrm{~m}, 1 \mathrm{H}, \mathrm{H}_{4}\right), 4.40-4.47\left(\mathrm{~m}, 3 \mathrm{H}, 2 \times \mathrm{H}_{5}\right.$, $\left.\mathrm{H}_{4^{\prime}}\right), 4.77\left(\mathrm{dd}, J=11.0,10.1 \mathrm{~Hz}, 1 \mathrm{H}, \mathrm{H}_{3^{\prime}}\right) ;{ }^{13} \mathrm{C}$ NMR $(150 \mathrm{MHz}$, $\left.\mathrm{CDCl}_{3}\right) \delta 8.5\left(\mathrm{q}, \mathrm{C}_{5^{\prime}} \mathrm{CH}_{2} \underline{\mathrm{CH}_{3}}\right), 19.7\left(\mathrm{q}, \mathrm{C}_{2}, \underline{\mathrm{CH}_{3}}\right), 20.3\left(\mathrm{q}, \mathrm{C}_{6}{ }^{\prime} \mathrm{CH}_{3}\right)$, $24.8\left(\mathrm{t}, \mathrm{C}_{5^{\prime}} \underline{\mathrm{CH}_{2}} \mathrm{CH}_{3}\right), 36.6\left(\mathrm{~d}, \mathrm{C}_{4^{\prime}}\right), 42.9\left(\mathrm{t}, \mathrm{C}_{4}\right), 50.0\left(\mathrm{~d}, \mathrm{C}_{5^{\prime}}\right), 57.0$ $\left(\mathrm{d}, \mathrm{C}_{3^{\prime}}\right), 61.9\left(\mathrm{t}, \mathrm{C}_{5}\right), 76.1\left(\mathrm{~d}, \mathrm{C}_{6^{\prime}}\right), 77.0\left(\mathrm{~d}, \mathrm{C}_{2^{\prime}}\right), 153.0\left(\mathrm{~s}, \mathrm{C}_{2}\right), 173.2$ (s, $\mathrm{C}_{3^{\prime}}$ CO); MS (EI) $\mathrm{m} / z$ (relative intensity) 295 (M - oxazolidin-2-

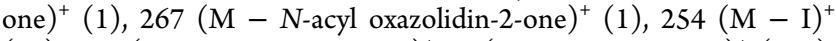
(33), $210\left(\mathrm{M}-\mathrm{CH}_{2} \mathrm{CH}_{2} \mathrm{I}-\mathrm{Me}\right)^{+}$or $(\mathrm{M}-\mathrm{Et}-\mathrm{I}-\mathrm{Me})^{+}(100)$, $168(\mathrm{M} \text { - I - oxazolidin-2-one })^{+}(2), 140(\mathrm{M}-\mathrm{I}-\mathrm{N}$-acyl oxazolidin-2-one $)^{+}(1)$; HRMS calcd for $\mathrm{C}_{13} \mathrm{H}_{20} \mathrm{NO}_{4}\left[(\mathrm{M}-\mathrm{I})^{+}\right]$ 254.1392, found 254.1389.

Synthesis of 2,3,4,5-Tetrasubstituted THFs 8 . These byproducts were punctually obtained during the synthesis of corresponding bicycles $\mathbf{5}$.

3-\{(2S*,3R*,4R*)-2-Methyl-4-[(E)-prop-1-en-1-yl]-1-oxaspiro[4.5]decane-3-carbonyl\}oxazolidin-2-one (8I). For the detailed synthetic procedure, see the synthesis of bicycle 51. THF 81 (7.8 $\mathrm{mg}, 19 \%, 80: 20 \mathrm{dr}$ ) was isolated as a white solid (probably crystalline): $R_{f}=0.43$ ( $n$-hexane/EtOAc $80: 20$, four times); ${ }^{1} \mathrm{H}$ NMR $\left(600 \mathrm{MHz}, \mathrm{CDCl}_{3}\right) \delta 1.09-1.14\left(\mathrm{~m}, 1 \mathrm{H}, \mathrm{CH}_{2}\right.$ from cyclohexane), $1.23-1.26\left(\mathrm{~m}, 1 \mathrm{H}, \mathrm{CH}_{2}\right.$ from cyclohexane), 1.28 (d, $\left.J=6.1 \mathrm{~Hz}, 3 \mathrm{H}, \mathrm{C}_{2}, \underline{\mathrm{Me}}\right), 1.39-1.45\left(\mathrm{~m}, 1 \mathrm{H}, \mathrm{CH}_{2}\right.$ from cyclohexane), 1.50-1.55 (m, $2 \mathrm{H}, \mathrm{C}_{2}$ from cyclohexane), $1.56-1.62\left(\mathrm{~m}, 5 \mathrm{H}, \mathrm{CH}_{2}\right.$ from cyclohexane), $1.62\left(\mathrm{dd}, J=6.0,1.0 \mathrm{~Hz}, 3 \mathrm{H}, \mathrm{C}_{4^{\prime}} \mathrm{CH}=\mathrm{CHMe}\right.$ ), $2.79\left(\mathrm{dd}, J=11.1,8.9 \mathrm{~Hz}, 1 \mathrm{H}, \mathrm{H}_{4^{\prime}}\right), 4.02\left(\mathrm{t}, J=8.0 \mathrm{~Hz}, 2 \mathrm{H}, \mathrm{H}_{4}\right), 4.20$ $\left(\mathrm{dq}, J=9.3,6.1 \mathrm{~Hz}, 1 \mathrm{H}, \mathrm{H}_{2^{\prime}}\right), 4.33-4.41\left(\mathrm{~m}, 3 \mathrm{H}, 2 \times \mathrm{H}_{5}, \mathrm{H}_{3^{\prime}}\right), 5.36-$ $5.48\left(\mathrm{~m}, 2 \mathrm{H}, \mathrm{C}_{4^{\prime}} \mathrm{C} \underline{\mathrm{H}}=\mathrm{C} \underline{\mathrm{HMe}}\right) ;{ }^{13} \mathrm{C} \mathrm{NMR}\left(150 \mathrm{MHz}, \mathrm{CDCl}_{3}\right) \delta 18.2$ $\left(\mathrm{q}, \mathrm{C}_{4^{\prime}} \mathrm{CH}=\mathrm{CHMe}\right), 21.0\left(\mathrm{q}, \mathrm{C}_{2^{\prime}} \underline{\mathrm{Me}}\right), 21.8\left(\mathrm{t}, \underline{\mathrm{CH}_{2}}\right.$ from cyclohexane), $23.3\left(\mathrm{t}, \underline{\mathrm{CH}}_{2}\right.$ from cyclohexane), $25.8\left(\mathrm{t}, \underline{\mathrm{CH}}_{2}\right.$ from cyclohexane), $34.5\left(\mathrm{t}, \underline{\mathrm{CH}}_{2}\right.$ from cyclohexane), $36.7\left(\mathrm{t}, \underline{\mathrm{C}} \mathrm{H}_{2}\right.$ from cyclohexane), $43.1\left(\mathrm{t}, \mathrm{C}_{4}\right), 53.7\left(\mathrm{~d}, \mathrm{C}_{3^{\prime}}\right), 60.9\left(\mathrm{~d}, \mathrm{C}_{4^{\prime}}\right), 61.8\left(\mathrm{t}, \mathrm{C}_{5}\right)$, $77.1\left(\mathrm{~d}, \mathrm{C}_{2^{\prime}}\right), 84.2\left(\mathrm{~s}, \mathrm{C}_{\mathrm{s}^{\prime}}\right), 128.1(\mathrm{~d},=\underline{\mathrm{C}} \mathrm{H}), 128.4(\mathrm{~d},=\underline{\mathrm{CH}}), 153.4$ (s, $\mathrm{N} \underline{\mathrm{C}}(\mathrm{O}) \mathrm{O}), 173.6\left(\mathrm{~s}, \mathrm{C}_{3^{\prime}} \underline{\mathrm{C}}(\mathrm{O}) \mathrm{N}\right)$; HRMS calcd for $\mathrm{C}_{17} \mathrm{H}_{25} \mathrm{NO}_{4} \mathrm{Na}$ $\left[(\mathrm{M}+\mathrm{Na})^{+}\right]$330.1681, found 330.1671.

3-\{(2S*,3R*,4R*,5R*)-2-Methyl-5-phenyl-4-[(E)-prop-1-en-1-yl]tetrahydrofuran-3-carbonyl\}oxazolidin-2-one (8m). Aldol $\mathbf{2 b}$ (110 $\mathrm{mg}, 0.48 \mathrm{mmol}$ ) was subjected to the general procedure for the synthesis of bicycles 5 (two-step EAP) and yielded, after purification by flash chromatography ( $28 \mathrm{~cm}$ of height of silica gel, $n$-hexane/ EtOAc 60:40), title compound $8 \mathrm{~m}(6 \mathrm{mg}, 4 \%, 80: 20 \mathrm{dr})$ and bicycle 5i (117 mg, 72\%, >95:5 dr). THF 8m was isolated as a thick colorless oil, and its description is given here. $R_{f}=0.42$ ( $n$-hexane/EtOAc 60:40); ${ }^{1} \mathrm{H} \mathrm{NMR}\left(500 \mathrm{MHz}, \mathrm{CDCl}_{3}\right) \delta 1.42(\mathrm{dd}, J=6.5,1.6 \mathrm{~Hz}, 3 \mathrm{H}$, $\left.\mathrm{C}_{4^{\prime}} \mathrm{CH}=\mathrm{CHCH}_{3}\right), 1.49\left(\mathrm{~d}, J=5.7 \mathrm{~Hz}, 3 \mathrm{H}, \mathrm{C}_{2} \mathrm{CH}_{3}\right), 3.46-3.50(\mathrm{~m}$, $\left.1 \mathrm{H}, \mathrm{H}_{4^{\prime}}\right), 4.02-4.07\left(\mathrm{~m}, 2 \mathrm{H}, \mathrm{H}_{4}\right), 4.27-4.33\left(\mathrm{~m}, 1 \mathrm{H}, \mathrm{H}_{3^{\prime}}\right), 4.36-4.47$ $\left(\mathrm{m}, 3 \mathrm{H}, \mathrm{H}_{5}, \mathrm{H}_{2^{\prime}}\right), 4.77-4.87\left(\mathrm{~m}, 1 \mathrm{H}, \mathrm{C}_{4^{\prime}} \mathrm{CH}=\mathrm{CHCH}_{3}\right), 5.21(\mathrm{~d}, J=$ $\left.8.3 \mathrm{~Hz}, 1 \mathrm{H}, \mathrm{H}_{5^{\prime}}\right), 5.29-5.37\left(\mathrm{~m}, 1 \mathrm{H}, \mathrm{C}_{4^{\prime}} \mathrm{CH}=\mathrm{CHCH}_{3}\right), 7.20-7.25$ $(\mathrm{m}, 3 \mathrm{H}, \mathrm{Ar}), 7.29-7.33(\mathrm{~m}, 2 \mathrm{H}, \mathrm{Ar}) ;{ }^{13} \mathrm{C} \mathrm{NMR}\left(150 \mathrm{MHz}, \mathrm{CDCl}_{3}\right) \delta$ $17.8\left(\mathrm{q}, \mathrm{C}_{4^{\prime}} \mathrm{CH}=\mathrm{CH}_{\underline{C}} \mathrm{H}_{3}\right), 19.7\left(\mathrm{q}, \mathrm{C}_{2^{\prime}} \underline{\mathrm{CH}}_{3}\right), 43.1\left(\mathrm{t}, \mathrm{C}_{4}\right), 53.8(\mathrm{~d}$, $\left.\mathrm{C}_{4^{\prime}}\right), 54.8\left(\mathrm{~d}, \mathrm{C}_{3^{\prime}}\right), 61.9\left(\mathrm{t}, \mathrm{C}_{5}\right), 79.2\left(\mathrm{~d}, \mathrm{C}_{2^{\prime}}\right), 83.8\left(\mathrm{~d}, \mathrm{C}_{5^{\prime}}\right), 127.0(\mathrm{~d}$, 2C, $\mathrm{Ar}$ ), 127.3 (d, Ar), 127.7 (d, $\left.\mathrm{C}_{4^{\prime}} \mathrm{CH}=\mathrm{CHCH}_{3}\right), 128.1$ (d, 2C, $\mathrm{Ar}), 129.0\left(\mathrm{~d}, \mathrm{C}_{4^{\prime}} \underline{\mathrm{C}} \mathrm{H}=\mathrm{CHCH}_{3}\right), 139.7(\mathrm{~s}, \mathrm{Ar}), 153.3\left(\mathrm{~s}, \mathrm{C}_{2}\right), 173.5$ $\left(\mathrm{s}, \mathrm{C}_{3^{\prime}} \mathrm{C}(\mathrm{O}) \mathrm{N}\right)$; HRMS calcd for $\mathrm{C}_{18} \mathrm{H}_{21} \mathrm{NO}_{4} \mathrm{Na}\left[(\mathrm{M}+\mathrm{Na})^{+}\right]$ 338.1368 , found 338.1369 .

3-\{(2S,3R,4R,5S)-5-(3-Fluorophenyl)-2-methyl-4-[(E)-prop-1-en1-yl]tetrahydrofuran-3-carbonyl\}oxazolidin-2-one (8n). Aldol $2 \mathrm{~b}$ ( $56 \mathrm{mg}, 0.25 \mathrm{mmol}$ ) was subjected to the general procedure for the synthesis of bicycles 5 (two-step EAP) and yielded, after purification by flash chromatography ( $28 \mathrm{~cm}$ of height of silica gel, $n$-hexane/ EtOAc 70:30), title compound $8 \mathrm{n}(7 \mathrm{mg}, 8 \%, 85: 15 \mathrm{dr})$ and bicycle 5n (56 mg, 64\%, >95:5 dr). THF 8n was isolated as a thick colorless 
oil, and its description is given here: $R_{f}=0.19$ and 0.29 ( $n$-hexane/ EtOAc 60:40); ${ }^{1} \mathrm{H}$ NMR (500 MHz, $\left.\mathrm{CDCl}_{3}\right) \delta 1.44(\mathrm{dd}, J=6.5,1.7$ $\left.\mathrm{Hz}, 3 \mathrm{H}, \mathrm{C}_{4^{\prime}} \mathrm{CH}=\mathrm{CHCH}_{3}\right), 1.49\left(\mathrm{~d}, J=5.9 \mathrm{~Hz}, 3 \mathrm{H}, \mathrm{C}_{2^{\prime}} \mathrm{CH}_{3}\right), 3.44-$ $3.50\left(\mathrm{~m}, 1 \mathrm{H}, \mathrm{H}_{4^{\prime}}\right), 4.05\left(\mathrm{t}, J=8.0 \mathrm{~Hz}, 2 \mathrm{H}, \mathrm{H}_{4}\right), 4.21-4.48(\mathrm{~m}, 4 \mathrm{H}, 2$ $\left.\times \mathrm{H}_{5}, \mathrm{H}_{2^{\prime}}, \mathrm{H}_{3^{\prime}}\right), 4.78-4.86\left(\mathrm{~m}, 1 \mathrm{H}, \mathrm{C}_{4^{\prime}} \mathrm{CH}=\mathrm{CHCH}_{3}\right), 5.19(\mathrm{~d}, J=$ $\left.8.0 \mathrm{~Hz}, 1 \mathrm{H}, \mathrm{H}_{5^{\prime}}\right), 5.30-5.38\left(\mathrm{~m}, 1 \mathrm{H}, \mathrm{C}_{4^{\prime}} \mathrm{CH}=\mathrm{CHCH}_{3}\right), 6.90-7.03$ (m, 4H, Ar), 7.10-7.15 (m, 1H, Ar) (once the ${ }^{1} \mathrm{H}$ NMR spectrum was recorded, the solvent was evaporated and the product was stored at $-18{ }^{\circ} \mathrm{C}$ under an $\mathrm{Ar}$ atmosphere; twelve months later, the NMR analysis showed that the product had suffered decomposition; thus, a well-resolved ${ }^{13} \mathrm{C}$ NMR spectrum could not be obtained); HRMS calcd for $\mathrm{C}_{18} \mathrm{H}_{20} \mathrm{NO}_{4} \mathrm{FNa}\left[(\mathrm{M}+\mathrm{Na})^{+}\right] 356.1274$, found 356.1281.

$3-\{(2 S * 3 R *, 4 R *, 5 S *)-5$-(2-Chlorophenyl)-2-methyl-4-[(E)-prop1-en-1-yl]tetrahydrofuran-3-carbonyl\}oxazolidin-2-one (80). Aldol $2 \mathbf{b}(102 \mathrm{mg}, 0.45 \mathrm{mmol})$ was subjected to the general procedure for the synthesis of bicycles 5 (two-step EAP) and yielded, after purification by flash chromatography $(21 \mathrm{~cm}$ of height of silica gel, $n$ hexane/EtOAc 70:30), title compound 8o (25 mg, 16\%, 80:20 dr), bicycle $50(100 \mathrm{mg}, 60 \%,>95: 5 \mathrm{dr})$, and bicycle $5 \mathbf{b}(1 \mathrm{mg}, 1 \%,>95: 5$ $\mathrm{dr})$. THF 80 was isolated as a thick yellowish oil, and its description is given here: $R_{f}=0.37$ ( $n$-hexane/EtOAc 60:40); ${ }^{1} \mathrm{H}$ NMR $(500 \mathrm{MHz}$, $\left.\mathrm{CDCl}_{3}\right) \delta 1.37\left(\mathrm{dd}, J=6.5,1.6 \mathrm{~Hz}, 3 \mathrm{H}, \mathrm{C}_{4}, \mathrm{CH}=\mathrm{CHCH}_{3}\right), 1.50(\mathrm{~d}, J$ $\left.=6.0 \mathrm{~Hz}, 3 \mathrm{H}, \mathrm{C}_{2^{\prime}} \mathrm{CH}_{3}\right), 3.62\left(\mathrm{dt}, J=9.7,7.3 \mathrm{~Hz}, 1 \mathrm{H}, \mathrm{H}_{4^{\prime}}\right), 4.07(\mathrm{t}, J=$ $\left.7.9 \mathrm{~Hz}, 2 \mathrm{H}, \mathrm{H}_{4}\right), 4.24\left(\mathrm{dd}, J=7.9,6.6 \mathrm{~Hz}, 1 \mathrm{H}, \mathrm{H}_{3^{\prime}}\right), 4.30(\mathrm{dq}, J=8.1$, $\left.6.0 \mathrm{~Hz}, 1 \mathrm{H}, \mathrm{H}_{2^{\prime}}\right), 4.37-4.44\left(\mathrm{~m}, 2 \mathrm{H}, \mathrm{H}_{5}\right), 4.87$ (ddq, $J=15.1,9.8,1.6$ $\left.\mathrm{Hz}, 1 \mathrm{H}, \mathrm{C}_{4^{\prime}} \mathrm{CH}=\mathrm{CHCH}_{3}\right), 5.34\left(\mathrm{dq}, J=14.9,6.3 \mathrm{~Hz}, 1 \mathrm{H}, \mathrm{C}_{4^{\prime}} \mathrm{CH}=\right.$ $\left.\mathrm{CHCH}_{3}\right), 5.51\left(\mathrm{~d}, J=7.6 \mathrm{~Hz}, 1 \mathrm{H}, \mathrm{H}_{5^{\prime}}\right), 7.15-7.19(\mathrm{~m}, 1 \mathrm{H}, \mathrm{Ar})$, 7.25-7.29 (m, 2H, Ar), 7.54-57 (m, 2H); ${ }^{1} \mathrm{H}$ NMR $(500 \mathrm{MHz}$, $\left.\mathrm{C}_{6} \mathrm{D}_{6}\right) \delta 1.26\left(\mathrm{dd}, J=6.5,1.6 \mathrm{~Hz}, 3 \mathrm{H}, \mathrm{C}_{4^{\prime}} \mathrm{CH}=\mathrm{CHC}_{3}\right), 1.61(\mathrm{~d}, J=$ $\left.6.1 \mathrm{~Hz}, 3 \mathrm{H}, \mathrm{C}_{2^{\prime}} \mathrm{CH}_{3}\right), 2.90-3.00\left(\mathrm{~m}, 4 \mathrm{H}, 2 \times \mathrm{H}_{4}, 2 \times \mathrm{H}_{5}\right), 3.99$ (ddd, $\left.J=9.6,6.9,6.9 \mathrm{~Hz}, 1 \mathrm{H}, \mathrm{H}_{4^{\prime}}\right), 4.44\left(\mathrm{dd}, J=7.7,6.1 \mathrm{~Hz}, 1 \mathrm{H}, \mathrm{H}_{2^{\prime}}\right), 4.62$ (dd, $J=7.7,6.4 \mathrm{~Hz}, 1 \mathrm{H}, \mathrm{H}_{3^{\prime}}$ ), 5.18 (ddd, $J=15.2,10.0,1.6 \mathrm{~Hz}, 1 \mathrm{H}$, $\left.\mathrm{C}_{4^{\prime}} \mathrm{CH}=\mathrm{CHCH}_{3}\right), 5.49\left(\mathrm{dq}, J=15.2,6.5 \mathrm{~Hz}, 1 \mathrm{H}, \mathrm{C}_{4^{\prime}} \mathrm{CH}=\right.$ $\left.\mathrm{CHCH}_{3}\right), 5.81\left(\mathrm{~d}, J=7.6 \mathrm{~Hz}, 1 \mathrm{H}, \mathrm{H}_{5^{\prime}}\right), 6.79(\mathrm{td}, J=7.7,1.6 \mathrm{~Hz}, 1 \mathrm{H}$, $\mathrm{Ar}), 6.99(\mathrm{td}, J=7.6,1.0 \mathrm{~Hz}, 1 \mathrm{H}, \mathrm{Ar}), 7.12(\mathrm{dd}, J=7.9,1.1 \mathrm{~Hz}, 1 \mathrm{H}$, Ar), 7.81 (dd, $J=7.8,1.4 \mathrm{~Hz}, 1 \mathrm{H}, \mathrm{Ar}) ;{ }^{13} \mathrm{C} \mathrm{NMR}\left(125 \mathrm{MHz}, \mathrm{CDCl}_{3}\right)$ $\delta 17.7\left(\mathrm{q}, \mathrm{C}_{4^{\prime}} \mathrm{CH}=\mathrm{CHCH}_{3}\right), 19.8\left(\mathrm{q}, \mathrm{C}_{2}{ }^{\prime} \underline{C H}_{3}\right), 43.1\left(\mathrm{t}, \mathrm{C}_{4}\right), 52.8(\mathrm{~d}$, $\left.\mathrm{C}_{4^{\prime}}\right), 54.8\left(\mathrm{~d}, \mathrm{C}_{3^{\prime}}\right), 61.9\left(\mathrm{t}, \mathrm{C}_{5}\right), 79.0\left(\mathrm{~d}, \mathrm{C}_{2^{\prime}}\right), 80.8\left(\mathrm{~d}, \mathrm{C}_{5^{\prime}}\right), 126.6(\mathrm{~d}$, $\mathrm{Ar}), 127.6\left(\mathrm{~d}, \mathrm{C}_{4^{\prime}} \mathrm{CH}=\mathrm{CHCH}_{3}\right), 127.9(\mathrm{~d}, \mathrm{Ar}), 128.3(\mathrm{~d}, \mathrm{Ar}), 128.6$ $\left(\mathrm{d}, \mathrm{C}_{4^{\prime}} \mathrm{CH}=\mathrm{CHCH}_{3}\right), 129.0$ (d, Ar), $131.8(\mathrm{~s}, \mathrm{Ar}), 137.3$ (s, Ar), $153.3\left(\mathrm{~s}, \mathrm{C}_{2}\right), 173.6\left(\mathrm{~s}, \mathrm{C}_{3^{\prime}} \underline{\mathrm{C}}(\mathrm{O}) \mathrm{N}\right) ; \mathrm{MS}(\mathrm{EI}) \mathrm{m} / z$ (relative intensity) $350(\mathrm{M}+1)^{+}(1), 349(\mathrm{M})^{+}(1), 308\left(\mathrm{M}-\mathrm{CH}_{3} \mathrm{CH}=\mathrm{CH}\right)^{+}(1), 262$ $(\mathrm{M}-1 \text {-oxazolidin-2-one })^{+}(2), 235(\mathrm{M}-\mathrm{N} \text {-acyl oxazolidin-2-one })^{+}$ (1), $193\left(\mathrm{M}-1-\mathrm{CH}_{3} \mathrm{CH}=\mathrm{CH}-\mathrm{N} \text {-acyl oxazolidin-2-one }\right)^{+}(24)$, $122(\mathrm{M}-2-\mathrm{Ar}-\mathrm{N} \text {-acyl oxazolidin-2-one })^{+}(100)$; HRMS calcd for $\mathrm{C}_{18} \mathrm{H}_{20} \mathrm{NO}_{4} \mathrm{Cl}\left[(\mathrm{M})^{+}\right]$349.1081, found 349.1097.

3-\{(2S,3R,4R,5S)-5-(4-Methoxyphenyl)-2-methyl-4-[(E)-prop-1en-1-yl]tetrahydrofuran-3-carbonyl\}oxazolidin-2-one (8q). Aldol 2b (119 $\mathrm{mg}, 0.53 \mathrm{mmol}$ ) was subjected to the general procedure for the synthesis of bicycles 5 (two-step EAP) and yielded, after purification by flash chromatography ( $28 \mathrm{~cm}$ of height of silica gel, $n$ hexane/EtOAc 60:40), title compound $\mathbf{8 q}(18 \mathrm{mg}, 10 \%, 80: 20 \mathrm{dr})$, bicycle $\mathbf{5 q}(119 \mathrm{mg}, 63 \%,>95: 5 \mathrm{dr})$, and bicycle $\mathbf{5 b}(8 \mathrm{mg}, 12 \%$, $>95: 5 \mathrm{dr}$ ). THF $\mathbf{8 q}$ was isolated as a thick yellowish oil, and its description is given here: $R_{f}=0.51$ ( $n$-hexane/EtOAc 60:40, two times); ${ }^{1} \mathrm{H}$ NMR $\left(500 \mathrm{MHz}, \mathrm{CDCl}_{3}\right) \delta 1.40(\mathrm{~d}, J=6.2 \mathrm{~Hz}, 3 \mathrm{H}$, $\left.\mathrm{C}_{4^{\prime}} \mathrm{CH}=\mathrm{CHCH}_{3}\right), 1.59\left(\mathrm{~d}, J=6.3 \mathrm{~Hz}, 3 \mathrm{H}, \mathrm{C}_{2^{\prime}} \mathrm{CH}_{3}\right), 3.15-3.20(\mathrm{~m}$, $\left.1 \mathrm{H}, \mathrm{H}_{4^{\prime}}\right), 3.80(\mathrm{~s}, 3 \mathrm{H}, \mathrm{MeO}), 4.05\left(\mathrm{t}, J=8.0 \mathrm{~Hz}, 2 \mathrm{H}, \mathrm{H}_{4}\right), 4.10-4.14$ $\left(\mathrm{m}, 1 \mathrm{H}, \mathrm{H}_{2^{\prime}}\right), 4.39-4.47\left(\mathrm{~m}, 3 \mathrm{H}, \mathrm{H}_{5}, \mathrm{H}_{3^{\prime}}\right), 4.72(\mathrm{~d}, J=9.4 \mathrm{~Hz}, 1 \mathrm{H}$, $\left.\mathrm{H}_{5^{\prime}}\right)$, 5.28-5.34 (m, $\left.1 \mathrm{H}, \mathrm{C}_{4^{\prime}} \mathrm{CH}=\mathrm{CHMe}\right), 5.40-5.45(\mathrm{~m}, 1 \mathrm{H}$, $\left.\mathrm{C}_{4}, \mathrm{CH}=\mathrm{CHMe}\right), 6.86(\mathrm{~d}, J=8.8 \mathrm{~Hz}, 2 \mathrm{H}, \mathrm{Ar}), 7.28(\mathrm{~d}, J=8.8 \mathrm{~Hz}$, $2 \mathrm{H}, \mathrm{Ar}) ;{ }^{13} \mathrm{C} \mathrm{NMR}\left(125 \mathrm{MHz}, \mathrm{CDCl}_{3}\right) \delta 18.1\left(\mathrm{q}, \mathrm{C}_{4^{\prime}} \mathrm{CH}=\mathrm{CHCH}_{3}\right)$, $20.6\left(\mathrm{q}, \mathrm{C}_{2^{\prime}} \mathrm{CH}_{3}\right), 43.1\left(\mathrm{t}, \mathrm{C}_{4}\right), 55.4(\mathrm{q}, \mathrm{MeO}), 55.6\left(\mathrm{~d}, \mathrm{C}_{3^{\prime}}\right), 58.6(\mathrm{~d}$, $\left.\mathrm{C}_{4^{\prime}}\right), 62.0\left(\mathrm{t}, \mathrm{C}_{5}\right), 80.9\left(\mathrm{~d}, \mathrm{C}_{2^{\prime}}\right), 85.2\left(\mathrm{~d}, \mathrm{C}_{5^{\prime}}\right), 113.8(\mathrm{~d}, 2 \mathrm{C}, \mathrm{Ar})$, 127.87 (d, 2C, Ar), 127.93 (d, $\mathrm{C}_{4^{\prime}} \mathrm{C} \underline{\mathrm{H}}=\mathrm{CHMe}$ ), 129.1 (d, $\mathrm{C}_{4^{\prime}} \mathrm{CH}=$ CHMe), 131.2 (s, Ar), 153.4 (s, $\mathrm{C}_{2}$ ), 159.3 (s, Ar), 172.9 (s, $\left.\mathrm{C}_{3^{\prime}} \mathrm{C}(\mathrm{O}) \mathrm{N}\right)$; HRMS calcd for $\mathrm{C}_{19} \mathrm{H}_{23} \mathrm{NO}_{5} \mathrm{Na}\left[(\mathrm{M}+\mathrm{Na})^{+}\right] 368.1474$, found 368.1485

3-\{(2S*,3R*,4R*,5R*)-5-(2-Methoxyphenyl)-2-methyl-4-[(E)prop-1-en-1-yl]tetrahydrofuran-3-carbonyl\}oxazolidin-2-one (8r).
For the detailed synthetic procedure, see the synthesis of bicycle $\mathbf{5 r}$. THF 8 r ( $3 \mathrm{mg}, 10 \%,>95: 5 \mathrm{dr})$ was isolated as a thick colorless oil, and its description is given here: $R_{f}=0.41$ ( $n$-hexane/EtOAc 60:40, two times); ${ }^{1} \mathrm{H}$ NMR $\left(500 \mathrm{MHz}, \mathrm{CDCl}_{3}\right) \delta 1.37$ (dd, $J=6.5,1.7 \mathrm{~Hz}$, $\left.3 \mathrm{H}, \mathrm{C}_{4^{\prime}} \mathrm{CH}=\mathrm{CHMe}\right), 1.48\left(\mathrm{~d}, J=5.8 \mathrm{~Hz}, 3 \mathrm{H}, \mathrm{C}_{2}, \mathrm{Me}\right), 3.49-3.54$ $\left(\mathrm{m}, 1 \mathrm{H}, \mathrm{H}_{4^{\prime}}\right), 3.75(\mathrm{~s}, 3 \mathrm{H}, \mathrm{MeO}), 4.03-4.07\left(\mathrm{~m}, 2 \mathrm{H}, \mathrm{H}_{4}\right), 4.22-4.29$ (m, $\left.1 \mathrm{H}, \mathrm{H}_{3^{\prime}}\right), 4.34-4.43\left(\mathrm{~m}, 3 \mathrm{H}, \mathrm{H}_{5}, \mathrm{H}_{2^{\prime}}\right), 4.89$ (ddq, $J=15.1$, 9.6, $\left.1.8 \mathrm{~Hz}, 1 \mathrm{H}, \mathrm{C}_{4^{\prime}} \mathrm{CH}=\mathrm{CHMe}\right), 5.21-5.31\left(\mathrm{~m}, 1 \mathrm{H}, \mathrm{C}_{4^{\prime}} \mathrm{CH}=\mathrm{CHMe}\right)$, $5.46\left(\mathrm{~d}, J=7.8 \mathrm{~Hz}, 1 \mathrm{H}, \mathrm{H}_{5^{\prime}}\right), 6.77-6.79(\mathrm{~m}, 1 \mathrm{H}, \mathrm{Ar}), 6.93-6.96(\mathrm{~m}$, $1 \mathrm{H}, \mathrm{Ar}), 7.18-7.22(\mathrm{~m}, 1 \mathrm{H}, \mathrm{Ar}), 7.41-7.43(\mathrm{~m}, 1 \mathrm{H}, \mathrm{Ar}) ;{ }^{13} \mathrm{C}$ NMR $\left(150 \mathrm{MHz}, \mathrm{CDCl}_{3}\right) \delta 17.7\left(\mathrm{q}, \mathrm{C}_{4}, \mathrm{CH}=\mathrm{CHCH}_{3}\right), 19.7\left(\mathrm{q}, \mathrm{C}_{2}, \underline{\mathrm{CH}_{3}}\right)$, $43.1\left(\mathrm{t}, \mathrm{C}_{4}\right), 53.3\left(\mathrm{~d}, \mathrm{C}_{4^{\prime}}\right), 54.6\left(\mathrm{~d}, \mathrm{C}_{3^{\prime}}\right), 55.2(\mathrm{q}, \mathrm{MeO}), 61.8\left(\mathrm{t}, \mathrm{C}_{5}\right)$, $78.8\left(\mathrm{~d}, \mathrm{C}_{2^{\prime}}\right), 79.3\left(\mathrm{~d}, \mathrm{C}_{5^{\prime}}\right), 109.8(\mathrm{~d}, \mathrm{Ar}), 120.4(\mathrm{~d}, \mathrm{Ar}), 126.4(\mathrm{~d}$, $\mathrm{C}_{4}, \mathrm{CH}=\mathrm{CHCH}_{3}$ ), 127.0 (d, Ar), 128.1 (d, Ar), 128.2 (s, Ar), 130.0 $\left(\mathrm{d}, \mathrm{C}_{4} \mathrm{CH}=\mathrm{CHCH}_{3}\right), 153.2\left(\mathrm{~s}, \mathrm{C}_{2}\right), 156.0(\mathrm{~s}, \mathrm{Ar}), 174.0(\mathrm{~s}$, $\left.\mathrm{C}_{3^{\prime}} \mathrm{C}(\mathrm{O}) \mathrm{N}\right) ; \mathrm{MS}(\mathrm{EI}) \mathrm{m} / z$ (relative intensity) $(\mathrm{M})^{+}(1), 303(\mathrm{M}-1$ $\left.-\mathrm{CH}_{3} \mathrm{CH}=\mathrm{CH}\right)^{+}(1), 259(\mathrm{M}-\text { oxazolidin-2-one })^{+}(1), 197(\mathrm{M}-$ $\left.\mathrm{Ar}-\mathrm{CH}_{3} \mathrm{CH}=\mathrm{CH}\right)^{+}(1), 122(\mathrm{M}-2-\mathrm{Ar}-\mathrm{N}$-acyl oxazolidin-2one $)^{+}(100)$; HRMS calcd for $\mathrm{C}_{19} \mathrm{H}_{23} \mathrm{NO}_{5} \mathrm{Na}\left[(\mathrm{M}+\mathrm{Na})^{+}\right] 368.1474$, found 368.1474 .

Ethyl $\left(R^{*}, E\right)-2-\left[\left(S^{*}\right)-1-H y d r o x y-3-m e t h y l b u t y l\right] h e x-3-e n o a t e$ (syn-9a) and Ethyl $\left(S^{*}, E\right)-2-\left[\left(S^{*}\right)-1-\right.$ Hydroxy-3-methylbutyl]hex-3-enoate (anti-9a). All the subsequent operations were performed under an $\mathrm{Ar}$ atmosphere. To an ice-cooled solution of $i$ $\mathrm{Pr}_{2} \mathrm{NH}$ ( $1.44 \mathrm{~mL}, 10.27 \mathrm{mmol}, 1.2$ equiv) in THF ( $43 \mathrm{~mL}, 0.2 \mathrm{M}$ with regard to the ester) was added a $2.5 \mathrm{M}$ solution of $n$-butyllithium in hexanes ( $3.8 \mathrm{~mL}, 9.5 \mathrm{mmol}, 1.1$ equiv). The mixture was stirred at $\mathrm{rt}$ for $15 \mathrm{~min}$ and then cooled to $-78^{\circ} \mathrm{C}$. A solution of commercial ethyl (E)-hex-3-enoate $(1.4 \mathrm{~mL}, 8.56 \mathrm{mmol})$ in THF $(43 \mathrm{~mL}, 0.2 \mathrm{M})$ was added dropwise, and the mixture was kept at that temperature for 30 min. After that, a solution of $i$-BuCHO $(1.1 \mathrm{~mL}, 10.27 \mathrm{mmol}, 1.2$ equiv) in THF ( $43 \mathrm{~mL}, 0.2 \mathrm{M}$ with regard to the ester) was added dropwise, and the mixture was allowed to warm to rt. After $12 \mathrm{~h}$, a saturated $\mathrm{NH}_{4} \mathrm{Cl}$ aqueous solution $(150 \mathrm{~mL})$ was added and the mixture was poured into a separatory funnel where the layers were separated. The aqueous layer was extracted with EtOAc $(3 \times 150$ $\mathrm{mL}$ ), and the combined organic layers were dried over anhydrous $\mathrm{MgSO}_{4}$, filtered, and concentrated to provide a 2.2:1 syn/anti aldol mixture $(69: 31 \mathrm{dr})$. Purification by flash chromatography $(28 \mathrm{~cm}$ of height of silica gel, $n$-hexane/EtOAc 95:5) allowed the isolation of aldols syn-9a (1.06 g, 57\%) and anti-9a (482 mg, 26\%), both as yellowish oils. syn-9a: $R_{f}=0.61$ ( $n$-hexane/EtOAc 80:20); ${ }^{1} \mathrm{H}$ NMR $\left(400 \mathrm{MHz}, \mathrm{CDCl}_{3}\right) \delta 0.88\left(\mathrm{~d}, J=6.8 \mathrm{~Hz}, 3 \mathrm{H}, \mathrm{C}_{3^{\prime}}\left(\mathrm{CH}_{3}\right)_{2}\right), 0.90(\mathrm{~d}, J$ $\left.=6.8 \mathrm{~Hz}, 3 \mathrm{H}, \mathrm{C}_{3^{\prime}}\left(\mathrm{CH}_{3}\right)_{2}\right), 0.99(\mathrm{t}, J=7.5 \mathrm{~Hz}, 3 \mathrm{H}), 1.10(\mathrm{ddd}, J=$ $\left.13.9,8.9,3.5 \mathrm{~Hz}, 1 \mathrm{H}, \mathrm{H}_{2^{\prime}}\right), 1.25\left(\mathrm{t}, J=7.2 \mathrm{~Hz}, 3 \mathrm{H}, \mathrm{CO}_{2} \mathrm{CH}_{2} \mathrm{CH}_{3}\right)$, 1.41 (ddd, $\left.J=14.0,9.5,5.1 \mathrm{~Hz}, 1 \mathrm{H}, \mathrm{H}_{2^{\prime}}\right), 1.73-1.84\left(\mathrm{~m}, 1 \mathrm{H}, \mathrm{H}_{3^{\prime}}\right)$, 2.04-2.11 (m, $\left.2 \mathrm{H}, \mathrm{H}_{5}\right), 2.60(\mathrm{br} \mathrm{s}, 1 \mathrm{H}, \mathrm{OH}), 2.92(\mathrm{dd}, J=9.1,4.7$ $\left.\mathrm{Hz}, 1 \mathrm{H}, \mathrm{H}_{2}\right), 3.90-3.94\left(\mathrm{~m}, 1 \mathrm{H}, \mathrm{H}_{1^{\prime}}\right), 4.15(\mathrm{q}, J=7.2 \mathrm{~Hz}, 2 \mathrm{H}$, $\left.\mathrm{CO}_{2} \mathrm{CH}_{2} \mathrm{CH}_{3}\right), 5.51\left(\mathrm{dd}, J=15.7,9.3 \mathrm{~Hz}, 1 \mathrm{H}, \mathrm{H}_{3}\right), 5.67(\mathrm{dt}, J=15.5$, $\left.6.5 \mathrm{~Hz}, 1 \mathrm{H}, \mathrm{H}_{4}\right) ;{ }^{13} \mathrm{C} \mathrm{NMR}\left(100 \mathrm{MHz}, \mathrm{CDCl}_{3}\right) \delta 13.6\left(\mathrm{q}, \mathrm{C}_{6}\right), 14.3$ $\left(\mathrm{q}, \mathrm{CO}_{2} \mathrm{CH}_{2} \mathrm{CH}_{3}\right), 21.9\left(\mathrm{q}, \mathrm{C}_{3^{\prime}}\left(\mathrm{CH}_{3}\right)_{2}\right), 23.6\left(\mathrm{q}, \mathrm{C}_{3^{\prime}}\left(\mathrm{CH}_{3}\right)_{2}\right), 24.5$ (d, $\left.\mathrm{C}_{3^{\prime}}\right), 25.8\left(\mathrm{t}, \mathrm{C}_{5}\right), 43.3\left(\mathrm{t}, \mathrm{C}_{2^{\prime}}\right), 55.4\left(\mathrm{~d}, \mathrm{C}_{2}\right), 60.9\left(\mathrm{t}, \mathrm{CO}_{2} \mathrm{CH}_{2} \mathrm{CH}_{3}\right)$, $66.7\left(\mathrm{~d}, \mathrm{C}_{1^{\prime}}\right), 122.3\left(\mathrm{~d}, \mathrm{C}_{3}\right), 138.6\left(\mathrm{~d}, \mathrm{C}_{4}\right), 174.1\left(\mathrm{~s}, \mathrm{C}_{1}\right) ; \mathrm{MS}(\mathrm{EI}) \mathrm{m} /$ $z$ (relative intensity) $211(\mathrm{M}-\mathrm{OH})^{+}(1), 171(\mathrm{M}-i-\mathrm{Bu})^{+}(1), 155$ $\left(\mathrm{M}-\mathrm{CO}_{2} \mathrm{Et}\right)^{+}(2), 142\left(\mathrm{M}+1-\mathrm{CH}(\mathrm{OH}) \mathrm{CH}_{2} \mathrm{CH}\left(\mathrm{CH}_{3}\right)_{2}\right)^{+}(100)$; HRMS calcd for $\mathrm{C}_{8} \mathrm{H}_{14} \mathrm{O}_{2}\left[\left(\mathrm{M}+1-\mathrm{CH}(\mathrm{OH}) \mathrm{CH}_{2} \mathrm{CH}\left(\mathrm{CH}_{3}\right)_{2}\right)^{+}\right]$ 142.0994, found 142.0990. anti-9a: $R_{f}=0.49$ ( $n$-hexane/EtOAc $80: 20) ;{ }^{1} \mathrm{H}$ NMR $\left(400 \mathrm{MHz}, \mathrm{CDCl}_{3}\right) \delta 0.89(\mathrm{~d}, J=6.8 \mathrm{~Hz}, 3 \mathrm{H}$, $\left.\mathrm{C}_{3^{\prime}}\left(\mathrm{CH}_{3}\right)_{2}\right), 0.91\left(\mathrm{~d}, J=6.8 \mathrm{~Hz}, 3 \mathrm{H}, \mathrm{C}_{3^{\prime}}\left(\mathrm{CH}_{3}\right)_{2}\right), 0.98(\mathrm{t}, J=7.5 \mathrm{~Hz}$, $3 \mathrm{H}), 1.22-1.35\left(\mathrm{~m}, 2 \mathrm{H}, \mathrm{H}_{2^{\prime}}\right), 1.26\left(\mathrm{t}, J=7.2 \mathrm{~Hz}, 3 \mathrm{H}, \mathrm{CO}_{2} \mathrm{CH}_{2} \mathrm{CH}_{3}\right)$, 1.79-1.89 (m, $\left.1 \mathrm{H}, \mathrm{H}_{3^{\prime}}\right), 2.01-2.09\left(\mathrm{~m}, 2 \mathrm{H}, \mathrm{H}_{5}\right), 2.40($ br s, $1 \mathrm{H}$, $\mathrm{OH}), 2.98\left(\mathrm{dd}, J=8.9,7.4 \mathrm{~Hz}, 1 \mathrm{H}, \mathrm{H}_{2}\right), 3.82-3.88\left(\mathrm{br} \mathrm{m}, 1 \mathrm{H}, \mathrm{H}_{1^{\prime}}\right)$, 4.13-4.20 (m, $\left.2 \mathrm{H}, \mathrm{CO}_{2} \mathrm{CH}_{2} \mathrm{CH}_{3}\right), 5.42(\mathrm{ddt}, J=15.4,9.0,1.5 \mathrm{~Hz}$, $\left.1 \mathrm{H}, \mathrm{H}_{3}\right), 5.67\left(\mathrm{dt}, J=15.4,6.4 \mathrm{~Hz}, 1 \mathrm{H}, \mathrm{H}_{4}\right) ;{ }^{13} \mathrm{C} \mathrm{NMR}(100 \mathrm{MHz}$, $\left.\mathrm{CDCl}_{3}\right) \delta 13.6\left(\mathrm{q}, \mathrm{C}_{6}\right), 14.3\left(\mathrm{q}, \mathrm{CO}_{2} \mathrm{CH}_{2} \mathrm{CH}_{3}\right), 21.7\left(\mathrm{q}, \mathrm{C}_{3^{\prime}}\left(\mathrm{CH}_{3}\right)_{2}\right)$, $23.8\left(\mathrm{q}, \mathrm{C}_{3^{\prime}}\left(\mathrm{CH}_{3}\right)_{2}\right), 24.6\left(\mathrm{~d}, \mathrm{C}_{3^{\prime}}\right), 25.7\left(\mathrm{t}, \mathrm{C}_{5}\right), 44.0\left(\mathrm{t}, \mathrm{C}_{2^{\prime}}\right), 56.4(\mathrm{~d}$, $\left.\mathrm{C}_{2}\right), 60.8\left(\mathrm{t}, \mathrm{CO}_{2} \mathrm{CH}_{2} \mathrm{CH}_{3}\right), 71.1\left(\mathrm{~d}, \mathrm{C}_{1^{\prime}}\right), 123.6\left(\mathrm{~d}, \mathrm{C}_{3}\right), 137.4(\mathrm{~d}$, $\left.\mathrm{C}_{4}\right), 174.0\left(\mathrm{~s}, \mathrm{C}_{1}\right) ; \mathrm{MS}(\mathrm{EI}) \mathrm{m} / z$ (relative intensity) $211(\mathrm{M}-\mathrm{OH})^{+}$ $(1), 171(\mathrm{M}-i-\mathrm{Bu})^{+}(2), 155\left(\mathrm{M}-\mathrm{CO}_{2} \mathrm{Et}\right)^{+}(2), 142(\mathrm{M}+1-$ 
$\left.\mathrm{CH}(\mathrm{OH}) \mathrm{CH}_{2} \mathrm{CH}\left(\mathrm{CH}_{3}\right)_{2}\right)^{+}$(100); HRMS calcd for $\mathrm{C}_{8} \mathrm{H}_{14} \mathrm{O}_{2}[(\mathrm{M}+$ $\left.\left.1-\mathrm{CH}(\mathrm{OH}) \mathrm{CH}_{2} \mathrm{CH}\left(\mathrm{CH}_{3}\right)_{2}\right)^{+}\right]$142.0994, found 142.0989.

Methyl $\left(R^{*}, E\right)-2-\left[\left(S^{*}\right)-1-\mathrm{Hydroxyethyl}\right] \mathrm{hex}$-3-enoate (syn9b). To a solution of aldol $\mathbf{2 b}(29 \mathrm{mg}, 0.13 \mathrm{mmol})$ in DCM (1.3 $\mathrm{mL}, 0.1 \mathrm{M}$ ) were sequentially added, under an Ar atmosphere, $\mathrm{MeOH}$ (0.11 mL, $2.60 \mathrm{mmol}, 20$ equiv) and $\mathrm{FeCl}_{3}(52.7 \mathrm{mg}, 0.33 \mathrm{mmol}, 2.5$ equiv). The reaction mixture was stirred for $16 \mathrm{~h}$, and then $\mathrm{H}_{2} \mathrm{O}$ was added. The mixture was poured into a separatory funnel where the layers were separated. The aqueous layer was extracted three times with DCM, and the combined organic layers were dried over anhydrous $\mathrm{MgSO}_{4}$, filtered, concentrated, and purified by flash chromatography (16 $\mathrm{cm}$ of height of silica gel, $n$-hexane/EtOAc $70: 30)$ to yield aldol syn-9b $(15 \mathrm{mg}, 70 \%)$ as a colorless oil: $R_{f}=0.43$ ( $n$-hexane/EtOAc 60:40, two times); ${ }^{1} \mathrm{H}$ NMR $\left(500 \mathrm{MHz}, \mathrm{CDCl}_{3}\right) \delta$ $1.00\left(\mathrm{t}, J=7.4 \mathrm{~Hz}, 3 \mathrm{H}, \mathrm{H}_{6}\right), 1.15\left(\mathrm{~d}, J=6.4 \mathrm{~Hz}, 3 \mathrm{H}, \mathrm{H}_{2^{\prime}}\right), 2.06-2.12$ (m, $\left.2 \mathrm{H}, \mathrm{H}_{5}\right), 2.59(\mathrm{br} \mathrm{s}, 1 \mathrm{H}, \mathrm{OH}), 2.94\left(\mathrm{dd}, J=9.2,5.2 \mathrm{~Hz}, 1 \mathrm{H}, \mathrm{H}_{2}\right)$, $3.70\left(\mathrm{~s}, 3 \mathrm{H}, \mathrm{CO}_{2} \mathrm{Me}\right), 4.00-4.05\left(\mathrm{~m}, 1 \mathrm{H}, \mathrm{H}_{1^{\prime}}\right), 5.51(\mathrm{dd}, J=15.2,9.4$ $\left.\mathrm{Hz}, 1 \mathrm{H}, \mathrm{H}_{3}\right), 5.70\left(\mathrm{dt}, J=15.2,6.4 \mathrm{~Hz}, 1 \mathrm{H}, \mathrm{H}_{4}\right) ;{ }^{13} \mathrm{C}$ NMR $(125$ $\left.\mathrm{MHz}_{\mathrm{CDCl}}\right) \delta 13.6\left(\mathrm{q}, \mathrm{C}_{6}\right), 20.1\left(\mathrm{q}, \mathrm{C}_{2^{\prime}}\right), 25.8\left(\mathrm{t}, \mathrm{C}_{5}\right), 52.0(\mathrm{q}$, $\mathrm{CO}_{2} \mathrm{Me}$ ), $56.4\left(\mathrm{~d}, \mathrm{C}_{2}\right), 67.9\left(\mathrm{~d}, \mathrm{C}_{1^{\prime}}\right), 122.3\left(\mathrm{~d}, \mathrm{C}_{3}\right), 139.0\left(\mathrm{~d}, \mathrm{C}_{4}\right)$, $174.3\left(\mathrm{~s}, \mathrm{C}_{1}\right)$; HRMS calcd for $\mathrm{C}_{9} \mathrm{H}_{16} \mathrm{O}_{3} \mathrm{Na}\left[(\mathrm{M}+\mathrm{Na})^{+}\right]$195.0997, found 195.0997 .

General Procedure for the Synthesis of 4-Halo-2,3,4,5,6pentasubstituted THPs 10. To a solution of the aldol and the aldehyde $\mathrm{R}^{3} \mathrm{CHO}$ ( 1.5 equiv) in DCM $(0.1 \mathrm{M})$ were sequentially added, under an $\mathrm{Ar}$ atmosphere, $\mathrm{TMSCl}$ (1 equiv) and $\mathrm{Fe}(\mathrm{acac})_{3}(0.1$ equiv). Once TLC analysis revealed full conversion of the starting material $(<30 \mathrm{~min})$, the reaction was quenched by adding $\mathrm{H}_{2} \mathrm{O}$ and the mixture was poured into a separatory funnel where the layers were separated. The aqueous layer was extracted three times with DCM, and the combined organic layers were dried over anhydrous $\mathrm{MgSO}_{4}$, filtered, concentrated, and purified by flash chromatography.

Ethyl $\left(2 S^{*}, 3 R^{*}, 4 S^{*}, 5 R^{*}, 6 R^{*}\right)-4$-Chloro-5-ethyl-2,6-diisobutyltetrahydro-2H-pyran-3-carboxylate (10a). syn-Aldol 9a (109 mg, $0.48 \mathrm{mmol}$ ) was subjected to the general procedure for the synthesis of 4-halo-2,3,4,5,6-pentasubstituted THPs 10 and yielded, after purification by flash chromatography $(28 \mathrm{~cm}$ of height of silica gel, $n$-hexane/EtOAc 98/:2), THP 10a (111 mg, 70\%, >95:5 dr). Alternatively, a solution of syn-aldol $9 \mathrm{a}(150 \mathrm{mg}, 0.66 \mathrm{mmol})$ and $i$ $\mathrm{BuCHO}(0.11 \mathrm{~mL}, 0.99 \mathrm{mmol}, 1.5$ equiv) in DCM $(6.6 \mathrm{~mL}, 0.1 \mathrm{M})$ was treated with $\mathrm{FeCl}_{3}(110 \mathrm{mg}, 0.66 \mathrm{mmol}, 1$ equiv) and stirred for $30 \mathrm{~min}$. Then, $\mathrm{H}_{2} \mathrm{O}(10 \mathrm{~mL})$ was added, and the aqueous layer was extracted with DCM $(3 \times 10 \mathrm{~mL})$. The combined organic layers were dried over $\mathrm{MgSO}_{4}$, filtered, concentrated, and purified as described above to yield title compound 10a (103 mg, 47\%, >95:5 dr) and undesired rearranged byproduct 11 (11 mg, 7\%). 4-Chloro-THP 10a was isolated as a white solid, and its description is given here: $R_{f}=$ 0.51 ( $n$-hexane/EtOAc 98:2); mp 56-60 ${ }^{\circ} \mathrm{C}$ (from DCM $/ n$-hexane); ${ }^{1} \mathrm{H}$ NMR $\left(400 \mathrm{MHz}, \mathrm{CDCl}_{3}\right) \delta 0.81-0.91\left(\mathrm{~m}, 15 \mathrm{H}, 5 \times \mathrm{CH}_{3}\right), 0.98-$ $1.04\left(\mathrm{~m}, 1 \mathrm{H}, \mathrm{H}_{1^{\prime}}\right), 1.27\left(\mathrm{t}, J=7.1 \mathrm{~Hz}, 3 \mathrm{H}, \mathrm{CO}_{2} \mathrm{CH}_{2} \mathrm{CH}_{3}\right), 1.31-1.42$ (m, $\left.2 \mathrm{H}, \mathrm{H}_{1^{\prime \prime}}\right), 1.44-1.62\left(\mathrm{~m}, 3 \mathrm{H}, \mathrm{H}_{5}, 1 \times \mathrm{H}_{1^{\prime}}, 1 \times \mathrm{H}_{1^{\prime \prime}}\right), 1.72-1.92$ $\left(\mathrm{m}, 3 \mathrm{H}, 1 \times \mathrm{H}_{1^{\prime \prime}}, \mathrm{H}_{2^{\prime}}, \mathrm{H}_{2^{\prime \prime}}\right), 2.56\left(\mathrm{dd}, J=10.3,10.3 \mathrm{~Hz}, 1 \mathrm{H}, \mathrm{H}_{3}\right), 3.28$ $\left(\mathrm{td}, J=10.2,2.4 \mathrm{~Hz}, 1 \mathrm{H}, \mathrm{H}_{6}\right), 3.41\left(\mathrm{td}, J=10.1,1.9 \mathrm{~Hz}, 1 \mathrm{H}, \mathrm{H}_{2}\right)$, 4.13-4.27 (m, 3H, H, $\left.\mathrm{CO}_{2} \mathrm{CH}_{2} \mathrm{CH}_{3}\right) ;{ }^{13} \mathrm{C} \mathrm{NMR}\left(150 \mathrm{MHz}, \mathrm{CDCl}_{3}\right)$ $\delta 8.7\left(\mathrm{q}, \mathrm{C}_{2^{\prime \prime}}\right), 14.4\left(\mathrm{q}, \mathrm{CO}_{2} \mathrm{CH}_{2} \mathrm{CH}_{3}\right), 20.0\left(\mathrm{t}, \mathrm{C}_{1^{\prime \prime}}\right), 21.0(\mathrm{q}$, $\left.\mathrm{CH}\left(\mathrm{CH}_{3}\right)_{2}\right), 21.1\left(\mathrm{q}, \mathrm{CH}\left(\mathrm{CH}_{3}\right)_{2}\right), 23.8\left(\mathrm{q}, \mathrm{CH}\left(\underline{\mathrm{CH}}_{3}\right)_{2}\right), 24.1$ (q, $\left.\mathrm{CH}\left(\mathrm{CH}_{3}\right)_{2}\right), 24.17\left(\mathrm{~d}, \mathrm{CH}\left(\mathrm{CH}_{3}\right)_{2}\right), 24.22\left(\mathrm{~d}, \underline{\mathrm{CH}}\left(\mathrm{CH}_{3}\right)_{2}\right), 42.2(\mathrm{t}$, $\left.\mathrm{C}_{1^{\prime \prime}}\right), 43.0\left(\mathrm{t}, \mathrm{C}_{1^{\prime}}\right), 48.6\left(\mathrm{~d}, \mathrm{C}_{5}\right), 59.4\left(\mathrm{~d}, \mathrm{C}_{3}\right), 61.1\left(\mathrm{t}, \mathrm{CO}_{2} \mathrm{CH}_{2} \mathrm{CH}_{3}\right)$, $62.4\left(\mathrm{~d}, \mathrm{C}_{4}\right), 76.3\left(\mathrm{~d}, \mathrm{C}_{2}\right), 77.1\left(\mathrm{~d}, \mathrm{C}_{6}\right), 171.7\left(\mathrm{~s}, \mathrm{CO}_{2} \mathrm{CH}_{2} \mathrm{CH}_{3}\right)$; HRMS calcd for $\mathrm{C}_{18} \mathrm{H}_{33} \mathrm{ClO}_{3} \mathrm{Na}\left[(\mathrm{M}+\mathrm{Na})^{+}\right] 357.1986$, found 357.1993.

Ethyl $(2 S *, 3 S *, 4 S *, 5 R *, 6 R *)-4$-Chloro-5-ethyl-2,6-diisobutyltetrahydro-2H-pyran-3-carboxylate (10b). anti-9a (98 mg, 0.43 $\mathrm{mmol}$ ) was subjected to the general procedure for the synthesis of 4-halo-2,3,4,5,6-pentasubstituted THPs 10 and yielded, after a reaction time of $21 \mathrm{~h}$ and purification by flash chromatography $(28$ $\mathrm{cm}$ of height of silica gel, $n$-hexane/EtOAc 99:1), title compound 10b $(28 \mathrm{mg}, 20 \%,>95: 5 \mathrm{dr})$ and undesired lactone $12(48 \mathrm{mg}, 62 \%)$ as result of the 2-oxonia-Cope rearrangement. 4-Chloro-THP $10 \mathrm{~b}$ was isolated as a white solid, and its description is given here: $R_{f}=0.71(n$ -
hexane/EtOAc 90:10); mp 38-44 ${ }^{\circ} \mathrm{C}$ (from DCM $/ n$-hexane); ${ }^{1} \mathrm{H}$ NMR $\left(500 \mathrm{MHz}, \mathrm{CDCl}_{3}\right) \delta 0.83-0.92\left(\mathrm{~m}, 15 \mathrm{H}, 5 \times \mathrm{CH}_{3}\right), 1.19-$ $1.25\left(\mathrm{~m}, 1 \mathrm{H}, \mathrm{H}_{1^{\prime}}\right), 1.28\left(\mathrm{t}, J=7.0 \mathrm{~Hz}, 3 \mathrm{H}, \mathrm{CO}_{2} \mathrm{CH}_{2} \mathrm{CH}_{3}\right), 1.30-1.35$ (m, $\left.1 \mathrm{H}, \mathrm{H}_{1^{\prime \prime}}\right), 1.43-1.48\left(\mathrm{~m}, 1 \mathrm{H}, 1 \times \mathrm{H}_{1^{\prime}}\right), 1.53-1.59(\mathrm{~m}, 2 \mathrm{H}, 1 \times$ $\left.\mathrm{H}_{1^{\prime \prime}}, 1 \times \mathrm{H}_{1^{\prime \prime \prime}}\right), 1.67-1.73\left(\mathrm{~m}, 1 \mathrm{H}, 1 \times \mathrm{H}_{1^{\prime \prime}}\right), 1.79-1.86\left(\mathrm{~m}, 1 \mathrm{H}, \mathrm{H}_{2^{\prime}}\right)$, $1.89-1.96\left(\mathrm{~m}, 1 \mathrm{H}, \mathrm{H}_{2^{\prime \prime}}\right), 2.42\left(\mathrm{ddt}, J=11.2,11.2,3.7 \mathrm{~Hz}, 1 \mathrm{H}, \mathrm{H}_{5}\right)$, $2.97\left(\mathrm{dd}, J=5.3,2.7 \mathrm{~Hz}, 1 \mathrm{H}, \mathrm{H}_{3}\right), 3.20\left(\mathrm{td}, J=10.3,1.9 \mathrm{~Hz}, 1 \mathrm{H}, \mathrm{H}_{6}\right)$, $3.49\left(\mathrm{dt}, J=9.9,3.3 \mathrm{~Hz}, 1 \mathrm{H}, \mathrm{H}_{2}\right), 4.09\left(\mathrm{dd}, J=11.5,5.5 \mathrm{~Hz}, 1 \mathrm{H}, \mathrm{H}_{4}\right)$, 4.15-4.26 (m, 2H, $\left.\mathrm{CO}_{2} \mathrm{CH}_{2} \mathrm{CH}_{3}\right) ;{ }^{13} \mathrm{C}$ NMR (150 MHz, $\left.\mathrm{CDCl}_{3}\right) \delta$ $8.7\left(\mathrm{q}, \mathrm{C}_{2^{\prime \prime}}\right), 14.5\left(\mathrm{q}, \mathrm{CO}_{2} \mathrm{CH}_{2} \mathrm{CH}_{3}\right), 20.2\left(\mathrm{t}, \mathrm{C}_{1^{\prime \prime}}\right), 21.1(\mathrm{q}$, $\left.\mathrm{CH}\left(\underline{\mathrm{CH}}_{3}\right)_{2}\right), 21.7\left(\mathrm{q}, \mathrm{CH}\left(\underline{\mathrm{CH}}_{3}\right)_{2}\right), 23.4\left(\mathrm{q}, \mathrm{CH}\left(\mathrm{CH}_{3}\right)_{2}\right), 24.0$ (q, $\left.\mathrm{CH}\left(\mathrm{CH}_{3}\right)_{2}\right), 24.1$ (d, $\left.\underline{\mathrm{CH}}\left(\mathrm{CH}_{3}\right)_{2}\right), 24.5\left(\mathrm{~d}, \underline{\mathrm{CH}}\left(\mathrm{CH}_{3}\right)_{2}\right), 42.1$ (t, $\left.\mathrm{C}_{1^{\prime \prime}}\right), 42.5\left(\mathrm{t}, \mathrm{C}_{1^{\prime}}\right), 43.4\left(\mathrm{~d}, \mathrm{C}_{5}\right), 53.1\left(\mathrm{~d}, \mathrm{C}_{3}\right), 61.5\left(\mathrm{t}, \mathrm{CO}_{2} \mathrm{CH}_{2} \mathrm{CH}_{3}\right)$, $61.4\left(\mathrm{~d}, \mathrm{C}_{4}\right), 75.5\left(\mathrm{~d}, \mathrm{C}_{2}\right), 78.2\left(\mathrm{~d}, \mathrm{C}_{6}\right), 169.9\left(\mathrm{~s}, \mathrm{CO}_{2} \mathrm{CH}_{2} \mathrm{CH}_{3}\right)$; HRMS calcd for $\mathrm{C}_{18} \mathrm{H}_{33} \mathrm{ClO}_{3} \mathrm{Na}\left[(\mathrm{M}+\mathrm{Na})^{+}\right] 357.1986$, found 357.1986.

Methyl (2S*,3R*,4S*,5R*,6R*)-6-Butyl-4-chloro-5-ethyl-2-methyltetrahydro-2H-pyran-3-carboxylate (10C) and Methyl $\left(2 S^{*}, 3 R^{*}, 4 S^{*}, 5 R^{*}, 6 R^{*}\right)-2,6$-Dibutyl-4-chloro-5-ethyltetrahydro- $2 \mathrm{H}$ pyran-3-carboxylate (10d). syn-Aldol $9 \mathrm{~b}(53 \mathrm{mg}, 0.31 \mathrm{mmol})$ was subjected to the general procedure for the synthesis of 4-halo2,3,4,5,6-pentasubstituted THPs 10 and yielded, after purification by flash chromatography ( $28 \mathrm{~cm}$ of height of silica gel, $n$-hexane/EtOAc 98:2), undesired THP 10d (6 mg, 16\%, >95:5 dr) and expected THP 10c $(17 \mathrm{mg}, 20 \%,>95: 5 \mathrm{dr})$, both as colorless oils. 10c: $R_{f}=0.29(n-$ hexane/EtOAc 95:5); ${ }^{1} \mathrm{H}$ NMR $\left(600 \mathrm{MHz}, \mathrm{CDCl}_{3}\right) \delta 0.87(\mathrm{t}, J=7.5$ $\left.\mathrm{Hz}, 3 \mathrm{H}, \mathrm{H}_{2^{\prime \prime}}\right), 0.90\left(\mathrm{t}, J=7.0 \mathrm{~Hz}, 3 \mathrm{H}, \mathrm{H}_{4^{\prime \prime}}\right), 1.18(\mathrm{~d}, J=6.1 \mathrm{~Hz}, 3 \mathrm{H}$, $\left.\mathrm{H}_{1^{\prime}}\right), 1.25-1.36\left(\mathrm{~m}, 3 \mathrm{H}, 1 \times \mathrm{H}_{2^{\prime \prime}}, \mathrm{H}_{3^{\prime \prime}}\right), 1.38-1.44\left(\mathrm{~m}, 1 \mathrm{H}, \mathrm{H}_{1^{\prime \prime}}\right)$, $1.45-1.50\left(\mathrm{~m}, 1 \mathrm{H}, \mathrm{H}_{2^{\prime \prime}}\right), 1.53-1.57\left(\mathrm{~m}, 1 \mathrm{H}, \mathrm{H}_{5}\right), 1.57-1.62(\mathrm{~m}, 1 \mathrm{H}$, $\left.\mathrm{H}_{1^{\prime \prime}}\right), 1.63-1.68\left(\mathrm{~m}, 1 \mathrm{H}, \mathrm{H}_{1^{\prime \prime}}\right), 1.76-1.83\left(\mathrm{~m}, 1 \mathrm{H}, \mathrm{H}_{1^{\prime \prime}}\right), 2.58(\mathrm{dd}, J=$ 10.1, $\left.10.1 \mathrm{~Hz}, 1 \mathrm{H}, \mathrm{H}_{3}\right), 3.25-3.28\left(\mathrm{~m}, 1 \mathrm{H}, \mathrm{H}_{6}\right), 3.49(\mathrm{dq}, J=9.8,6.2$ $\left.\mathrm{Hz}, 1 \mathrm{H}, \mathrm{H}_{2}\right), 3.75\left(\mathrm{~s}, 3 \mathrm{H}, \mathrm{CO}_{2} \underline{\mathrm{Me}}\right), 4.20(\mathrm{dd}, J=11.0,11.0 \mathrm{~Hz}, 1 \mathrm{H}$, $\left.\mathrm{H}_{4}\right) ;{ }^{13} \mathrm{C}$ NMR $\left(150 \mathrm{MHz}, \mathrm{CDCl}_{3}\right) \delta 8.9\left(\mathrm{q}, \mathrm{C}_{2^{\prime \prime}}\right), 14.2\left(\mathrm{q}, \mathrm{C}_{4^{\prime \prime}}\right)$, $20.02\left(\mathrm{q}, \mathrm{C}_{1^{\prime}}\right), 20.07\left(\mathrm{t}, \mathrm{C}_{1^{\prime \prime}}\right), 22.8\left(\mathrm{t}, \mathrm{C}_{3^{\prime \prime \prime}}\right), 27.6\left(\mathrm{t}, \mathrm{C}_{2^{\prime \prime \prime}}\right), 32.7(\mathrm{t}$, $\left.\mathrm{C}_{1^{\prime \prime \prime}}\right), 47.6\left(\mathrm{~d}, \mathrm{C}_{5}\right), 52.2\left(\mathrm{q}, \mathrm{CO}_{2} \underline{\mathrm{Me}}\right), 60.1\left(\mathrm{~d}, \mathrm{C}_{3}\right), 62.1\left(\mathrm{~d}, \mathrm{C}_{4}\right), 74.4$

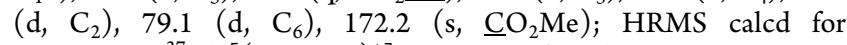
$\mathrm{C}_{14} \mathrm{H}_{25} \mathrm{O}_{3} \mathrm{Na}^{37} \mathrm{Cl}\left[(\mathrm{M}+\mathrm{Na})^{+}\right]$301.1360, found 301.1362. 10d: $R_{f}$ $=0.34\left(n\right.$-hexane $/$ EtOAc 95:5); ${ }^{1} \mathrm{H}$ NMR $\left(600 \mathrm{MHz}, \mathrm{CDCl}_{3}\right) \delta 0.871$ $\left(\mathrm{t}, J=7.6 \mathrm{~Hz}, 3 \mathrm{H}, \mathrm{H}_{2^{\prime \prime}}\right), 0.874\left(\mathrm{t}, J=7.2 \mathrm{~Hz}, 3 \mathrm{H}, \mathrm{H}_{4^{\prime}}\right.$ or $\left.\mathrm{H}_{4^{\prime \prime}}\right), 0.91(\mathrm{t}$, $J=7.1 \mathrm{~Hz}, 3 \mathrm{H}, \mathrm{H}_{4^{\prime}}$ or $\left.\mathrm{H}_{4^{\prime \prime}}\right), 1.25-1.37\left(\mathrm{~m}, 7 \mathrm{H}, 1 \times \mathrm{H}_{2^{\prime}}, 2 \times \mathrm{H}_{3^{\prime}}, 2 \times\right.$ $\left.\mathrm{H}_{2^{\prime \prime}}, 2 \times \mathrm{H}_{3^{\prime \prime \prime}}\right), 1.38-1.44\left(\mathrm{~m}, 1 \mathrm{H}, \mathrm{H}_{1^{\prime \prime}}\right), 1.45-1.52\left(\mathrm{~m}, 3 \mathrm{H}, 2 \times \mathrm{H}_{1^{\prime}}\right.$, $\left.1 \times \mathrm{H}_{2^{\prime}}\right), 1.52-1.55\left(\mathrm{~m}, 1 \mathrm{H}, \mathrm{H}_{5}\right), 1.58-1.62\left(\mathrm{~m}, 1 \mathrm{H}, \mathrm{H}_{1^{\prime \prime}}\right), 1.64-1.69$ $\left(\mathrm{m}, 1 \mathrm{H}, \mathrm{H}_{1^{\prime \prime}}\right), 1.75-1.82\left(\mathrm{~m}, 1 \mathrm{H}, \mathrm{H}_{1^{\prime \prime}}\right), 2.63(\mathrm{dd}, J=10.2,10.2 \mathrm{~Hz}$, $\left.1 \mathrm{H}, \mathrm{H}_{3}\right), 3.22\left(\mathrm{td}, J=9.6,2.5 \mathrm{~Hz}, 1 \mathrm{H}, \mathrm{H}_{6}\right), 3.33(\mathrm{td}, J=9.4,2.6 \mathrm{~Hz}$, $\left.1 \mathrm{H}, \mathrm{H}_{2}\right), 3.75\left(\mathrm{~s}, 3 \mathrm{H}, \mathrm{CO}_{2} \mathrm{Me}\right.$ ), $4.21\left(\mathrm{dd}, J=11.0,11.0 \mathrm{~Hz}, 1 \mathrm{H}, \mathrm{H}_{4}\right)$; ${ }^{13} \mathrm{C}$ NMR $\left(150 \mathrm{MHz}, \mathrm{CDCl}_{3}\right) \delta 8.9\left(\mathrm{q}, \mathrm{C}_{2^{\prime \prime}}\right), 14.1\left(\mathrm{q}, \mathrm{C}_{4^{\prime}}\right.$ or $\left.\mathrm{C}_{4^{\prime \prime}}\right)$, $14.2\left(\mathrm{q}, \mathrm{C}_{4^{\prime}}\right.$ or $\left.\mathrm{C}_{4^{\prime \prime}}\right), 20.1\left(\mathrm{t}, \mathrm{C}_{1^{\prime \prime}}\right), 22.5\left(\mathrm{t}, \mathrm{C}_{3^{\prime}}\right.$ or $\left.\mathrm{C}_{3^{\prime \prime}}\right), 22.6\left(\mathrm{t}, \mathrm{C}_{3^{\prime}}\right.$ or $\left.\mathrm{C}_{3^{\prime \prime \prime}}\right), 27.5\left(\mathrm{t}, \mathrm{C}_{2^{\prime}}\right.$ or $\left.\mathrm{C}_{2^{\prime \prime \prime}}\right), 27.7\left(\mathrm{t}, \mathrm{C}_{2^{\prime}}\right.$ or $\left.\mathrm{C}_{2^{\prime \prime \prime}}\right), 32.7\left(\mathrm{t}, \mathrm{C}_{1^{\prime \prime \prime}}\right), 33.8(\mathrm{t}$, $\left.\mathrm{C}_{1^{\prime}}\right), 48.2\left(\mathrm{~d}, \mathrm{C}_{5}\right), 52.2\left(\mathrm{q}, \mathrm{CO}_{2} \mathrm{Me}\right), 59.0\left(\mathrm{~d}, \mathrm{C}_{3}\right), 62.5\left(\mathrm{~d}, \mathrm{C}_{4}\right), 78.0$

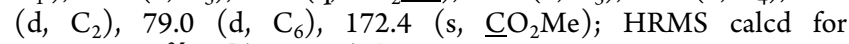
$\mathrm{C}_{17} \mathrm{H}_{31} \mathrm{O}_{3} \mathrm{Na}^{35} \mathrm{Cl}\left[(\mathrm{M}+\mathrm{Na})^{+}\right] 341.1859$, found 341.1865.

Ethyl $\left(4 S^{*}, 5 R^{*}, E\right)-4-E t h y l-5-h y d r o x y-7-m e t h y l o c t-2-e n o a t e$ (11). This undesired byproduct was obtained during the $\mathrm{FeCl}_{3}$ mediated synthesis of previously described 4-chloro-THP 10a (see the synthetic procedure in its section). 11 (11 mg, 7\%) was obtained as a colorless oil, and its description is given here: $R_{f}=0.17(n$ hexane/EtOAc 90:10); ${ }^{1} \mathrm{H}$ NMR (400 MHz, $\left.\mathrm{CDCl}_{3}\right) \delta 0.88(\mathrm{t}, J=$ $\left.7.7 \mathrm{~Hz}, 3 \mathrm{H}, \mathrm{H}_{2^{\prime}}\right), 0.91\left(\mathrm{~d}, J=6.5 \mathrm{~Hz}, 3 \mathrm{H}, \mathrm{CH}\left(\mathrm{CH}_{3}\right)_{2}\right), 0.92(\mathrm{~d}, J=$ $\left.6.6 \mathrm{~Hz}, 3 \mathrm{H}, \mathrm{CH}\left(\mathrm{CH}_{3}\right)_{2}\right), 1.17-1.25\left(\mathrm{~m}, 1 \mathrm{H}, \mathrm{H}_{6}\right), 1.30(\mathrm{t}, J=7.1 \mathrm{~Hz}$, $\left.3 \mathrm{H}, \mathrm{CO}_{2} \mathrm{CH}_{2} \mathrm{CH}_{3}\right), 1.34-1.40\left(\mathrm{~m}, 1 \mathrm{H}, \mathrm{H}_{6}\right), 1.43-1.53\left(\mathrm{~m}, 1 \mathrm{H}, \mathrm{H}_{1^{\prime}}\right)$, $1.56-1.66\left(\mathrm{~m}, 1 \mathrm{H}, \mathrm{H}_{1^{\prime}}\right), 1.71-1.82\left(\mathrm{~m}, 1 \mathrm{H}, \mathrm{H}_{7}\right), 2.02-2.09(\mathrm{~m}, 1 \mathrm{H}$, $\left.\mathrm{H}_{4}\right), 3.70-3.74\left(\mathrm{~m}, 1 \mathrm{H}, \mathrm{H}_{5}\right), 4.20\left(\mathrm{q}, J=7.1 \mathrm{~Hz}, 2 \mathrm{H}, \mathrm{CO}_{2} \mathrm{CH}_{2} \mathrm{CH}_{3}\right)$, $5.85\left(\mathrm{~d}, J=15.8 \mathrm{~Hz}, 1 \mathrm{H}, \mathrm{H}_{2}\right), 6.85\left(\mathrm{~d}, J=15.7,9.7 \mathrm{~Hz}, 1 \mathrm{H}, \mathrm{H}_{3}\right) ;{ }^{13} \mathrm{C}$ NMR (100 MHz, $\left.\mathrm{CDCl}_{3}\right) \delta 12.1\left(\mathrm{q}, \mathrm{C}_{2^{\prime}}\right), 14.4\left(\mathrm{q}, \mathrm{CO}_{2} \mathrm{CH}_{2} \mathrm{CH}_{3}\right)$, $22.0\left(\mathrm{q}, \mathrm{CH}\left(\mathrm{CH}_{3}\right)_{2}\right), 23.7\left(\mathrm{q}, \mathrm{CH}\left(\mathrm{CH}_{3}\right)_{2}\right), 23.9\left(\mathrm{t}, \mathrm{C}_{1^{\prime}}\right), 24.7\left(\mathrm{~d}, \mathrm{C}_{7}\right)$, $44.6\left(\mathrm{t}, \mathrm{C}_{6}\right), 51.1\left(\mathrm{~d}, \mathrm{C}_{4}\right), 60.5\left(\mathrm{t}, \mathrm{CO}_{2} \mathrm{CH}_{2} \mathrm{CH}_{3}\right), 71.7\left(\mathrm{~d}, \mathrm{C}_{5}\right), 123.9$ $\left(\mathrm{d}, \mathrm{C}_{2}\right), 149.0\left(\mathrm{~d}, \mathrm{C}_{3}\right), 166.5\left(\mathrm{~s}, \underline{\mathrm{CO}_{2}} \mathrm{CH}_{2} \mathrm{CH}_{3}\right)$; HRMS calcd for $\mathrm{C}_{13} \mathrm{H}_{24} \mathrm{O}_{3} \mathrm{Na}\left[(\mathrm{M}+\mathrm{Na})^{+}\right] 251.1623$, found 251.1624.

$\left(5 R^{*}, 6 S^{*}\right)-5$-Ethyl-6-isobutyl-5,6-dihydro-2H-pyran-2-one (12). This undesired byproduct was obtained during the synthesis of 
previously described 4-chloro-THP $10 \mathbf{b}$ (see the synthetic procedure in its section). 12 (48 $\mathrm{mg}, 62 \%)$ was obtained as a colorless oil, and its description is given here: $R_{f}=0.27\left(n\right.$-hexane/EtOAc 90:10); ${ }^{1} \mathrm{H}$ $\operatorname{NMR}\left(400 \mathrm{MHz}, \mathrm{CDCl}_{3}\right) \delta 0.90\left(\mathrm{~d}, J=6.4 \mathrm{~Hz}, 3 \mathrm{H}, \mathrm{CH}_{2} \mathrm{CH}\left(\mathrm{CH}_{3}\right)_{2}\right)$, $0.91\left(\mathrm{~d}, J=6.6 \mathrm{~Hz}, 3 \mathrm{H}, \mathrm{CH}_{2} \mathrm{CH}\left(\mathrm{CH}_{3}\right)_{2}\right), 0.96(\mathrm{t}, J=7.4 \mathrm{~Hz}, 3 \mathrm{H}$, $\left.\mathrm{CH}_{2} \mathrm{CH}_{3}\right), 1.32-1.39\left(\mathrm{~m}, 1 \mathrm{H}, \mathrm{CH}_{2} \mathrm{CH}\left(\mathrm{CH}_{3}\right)_{2}\right), 1.41-1.50(\mathrm{~m}, 1 \mathrm{H}$, $\left.\mathrm{CH}_{2} \mathrm{CH}_{3}\right), 1.57-1.65\left(\mathrm{~m}, 1 \mathrm{H}, \mathrm{CH}_{2} \mathrm{CH}_{3}\right), 1.67-1.75(\mathrm{~m}, 1 \mathrm{H}$, $\left.\mathrm{CH}_{2} \mathrm{CH}\left(\mathrm{CH}_{3}\right)_{2}\right), 1.86-1.97\left(\mathrm{~m}, 1 \mathrm{H}, \mathrm{CH}_{2} \mathrm{CH}\left(\mathrm{CH}_{3}\right)_{2}\right), 2.16-2.22$ $\left(\mathrm{m}, 1 \mathrm{H}, \mathrm{H}_{5}\right), 4.28$ (ddd, $\left.J=10.3,7.4,4.4 \mathrm{~Hz}, 1 \mathrm{H}, \mathrm{H}_{6}\right), 5.95(\mathrm{dd}, J=$ $\left.9.9,1.9 \mathrm{~Hz}, 1 \mathrm{H}, \mathrm{H}_{3}\right), 6.75\left(\mathrm{dd}, J=9.8,3.5 \mathrm{~Hz}, 1 \mathrm{H}, \mathrm{H}_{4}\right) ;{ }^{13} \mathrm{C}$ NMR $\left(100 \mathrm{MHz}, \mathrm{CDCl}_{3}\right) \delta 10.6\left(\mathrm{q}, \mathrm{CH}_{2} \underline{\mathrm{CH}}_{3}\right), 21.6\left(\mathrm{q}, \mathrm{CH}_{2} \mathrm{CH}\left(\mathrm{CH}_{3}\right)_{2}\right)$, $23.5\left(\mathrm{q}, \mathrm{CH}_{2} \mathrm{CH}\left(\mathrm{CH}_{3}\right)_{2}\right), 24.1\left(\mathrm{~d}, \mathrm{CH}_{2} \mathrm{CH}\left(\mathrm{CH}_{3}\right)_{2}\right), 24.3$ (t, $\left.\mathrm{CH}_{2} \mathrm{CH}_{3}\right), 39.9\left(\mathrm{~d}, \mathrm{C}_{5}\right), 42.5\left(\mathrm{t}, \underline{\mathrm{CH}}_{2} \mathrm{CH}\left(\mathrm{CH}_{3}\right)_{2}\right), 79.8\left(\mathrm{~d}, \mathrm{C}_{6}\right)$, 120.6 (d, $\mathrm{C}_{3}$ ), 149.1 (d, $\left.\mathrm{C}_{4}\right), 163.9\left(\mathrm{~s}, \mathrm{C}_{2}\right)$; MS (EI) $\mathrm{m} / z$ (relative intensity) $182(\mathrm{M})^{+}(1), 168(\mathrm{M}+1-\mathrm{Me})^{+}(1), 125(\mathrm{M}-i-\mathrm{Bu})^{+}$ (47), 96 (100); HRMS calcd for $\mathrm{C}_{11} \mathrm{H}_{18} \mathrm{O}_{2}\left[(\mathrm{M})^{+}\right]$182.1307, found 182.1300 .

Methyl $\left(2 S^{*}, 3 R^{*}, 5 S^{*}, 6 R^{*}\right)$-5-Ethyl-4-hydroxy-2,6-dimethyltetrahydro-2H-pyran-3-carboxylate (13). syn-Aldol $9 \mathrm{~b}(37 \mathrm{mg}$, $0.22 \mathrm{mmol}$ ) was subjected to the general procedure for the synthesis of bicycles 5 (two-step EAP). Once the reaction had reached completion, ${ }^{1} \mathrm{H}$ NMR analysis of the crude revealed a 1:1 mixture of the epimers at $\mathrm{C}_{4}$ of THP 13. After purification by flash chromatography $(21 \mathrm{~cm}$ of height of silica gel, $n$-hexane/EtOAc 95:5), that inseparable mixture of the isomers of $13(6 \mathrm{mg}, 12 \%$, $50: 50 \mathrm{dr}$ ) was isolated. The mixture decomposed after 1 month, in spite of being stored under $\mathrm{Ar}$ at $-18{ }^{\circ} \mathrm{C}$. 13: colorless oil; $R_{f}=0.49$ ( $n$-hexane/EtOAc 60:40); ${ }^{1} \mathrm{H}$ NMR $\left(400 \mathrm{MHz}, \mathrm{CDCl}_{3}\right) \delta 0.94(\mathrm{t}, J=$ $\left.7.6 \mathrm{~Hz}, 3 \mathrm{H}, \mathrm{H}_{2^{\prime \prime}}\right), 1.20\left(\mathrm{~d}, J=6.1 \mathrm{~Hz}, 3 \mathrm{H}, \mathrm{H}_{1^{\prime}}\right), 1.26(\mathrm{~d}, J=5.8 \mathrm{~Hz}$, $\left.3 \mathrm{H}, \mathrm{H}_{1^{\prime \prime}}\right), 1.40-1.55\left(\mathrm{~m}, 2 \mathrm{H}, \mathrm{H}_{5}, \mathrm{H}_{1^{\prime \prime}}\right), 1.61-1.71\left(\mathrm{~m}, 1 \mathrm{H}, \mathrm{H}_{1^{\prime \prime}}\right)$, 2.46-2.53 (m, $\left.1 \mathrm{H}, \mathrm{H}_{3}\right), 3.31-3.38\left(\mathrm{~m}, 1 \mathrm{H}, \mathrm{H}_{6}\right), 3.49-3.57(\mathrm{~m}, 1 \mathrm{H}$, $\left.\mathrm{H}_{2}\right), 3.75\left(\mathrm{~s}, 3 \mathrm{H}, \mathrm{CO}_{2} \mathrm{Me}\right), 4.67\left(\mathrm{dd}, J=10.3 \mathrm{~Hz}, 0.5 \mathrm{H}, \mathrm{H}_{4}\right), 4.80(\mathrm{dd}$, $\left.J=10.2 \mathrm{~Hz}, 0.5 \mathrm{H}, \mathrm{H}_{4}\right) ;{ }^{13} \mathrm{C} \mathrm{NMR}\left(125 \mathrm{MHz}, \mathrm{CDCl}_{3}\right) \delta 10.0\left(\mathrm{q}, \mathrm{C}_{2^{\prime \prime}}\right)$, $19.2\left(\mathrm{q}, \mathrm{C}_{1^{\prime \prime}}\right), 19.79\left(\mathrm{t}, \mathrm{C}_{1^{\prime \prime}}\right), 19.83\left(\mathrm{q}, \mathrm{C}_{1^{\prime}}\right), 47.7$ and $47.8\left(\mathrm{~d}, \mathrm{C}_{5}\right)$, $52.2\left(\mathrm{q}, \mathrm{CO}_{2} \mathrm{Me}\right), 56.5$ and $56.7\left(\mathrm{~d}, \mathrm{C}_{3}\right), 72.6$ and $72.7\left(\mathrm{~d}, \mathrm{C}_{2}\right), 74.4$ and 74.5 (d, $\left.\mathrm{C}_{6}\right), 92.4$ and 93.9 (d, $\left.\mathrm{C}_{4}\right), 172.3\left(\mathrm{~s}, \underline{\mathrm{CO}}_{2} \mathrm{Me}\right)$; HRMS calcd for $\mathrm{C}_{11} \mathrm{H}_{20} \mathrm{O}_{4} \mathrm{Na}\left[(\mathrm{M}+\mathrm{Na})^{+}\right] 239.1259$, found 239.1257 .

$\left(2 S^{*}, 5 S^{*}, 6 R^{*}\right)-5$-Ethyl- $N$-(2-hydroxyethyl)-2,6-diisobutyl5,6-dihydro-2H-pyran-3-carboxamide (14). A $1 \mathrm{M}$ solution of KHMDS in THF ( $0.76 \mathrm{~mL}, 0.76 \mathrm{mmol}, 1.5$ equiv) was added, at -78 ${ }^{\circ} \mathrm{C}$ and under an Ar atmosphere, dropwise to a stirred solution of bicycle 5a $(179 \mathrm{mg}, 0.50 \mathrm{mmol})$ in THF $(2.8 \mathrm{~mL}, 0.2 \mathrm{M})$. The reaction mixture was stirred at $-78{ }^{\circ} \mathrm{C}$ for $2 \mathrm{~h}$, until TLC analysis revealed full conversion of the starting material. Then, the cold bath was removed, the reaction quenched with a saturated $\mathrm{NH}_{4} \mathrm{Cl}$ aqueous solution $(5 \mathrm{~mL})$, and the mixture poured into a separatory funnel with $5 \mathrm{~mL}$ of DCM. The layers were separated; the aqueous layer was extracted with DCM $(3 \times 5 \mathrm{~mL})$, and the combined organic layers were dried over $\mathrm{MgSO}_{4}$, filtered, concentrated, and purified by flash chromatography (21 $\mathrm{cm}$ of height of silica gel, $n$-hexane/EtOAc $30: 70)$ to yield title compound $\mathbf{1 4}(73 \mathrm{mg}, 47 \%)$ as a colorless oil. Product 14 was revealed properly with oleum and with a phosphomolybdic acid, although it was not revealed with ninhydrin, vanillin, or anisaldehyde: $R_{f}=0.35$ (n-hexane/EtOAc 20:80); ${ }^{1} \mathrm{H}$ NMR $\left(500 \mathrm{MHz}, \mathrm{CDCl}_{3}\right) \delta 0.85-0.94\left(\mathrm{~m}, 15 \mathrm{H}, 2 \times\left(\mathrm{CH}_{3}\right)_{2} \mathrm{CHCH}_{2}\right.$, $\left.3 \times \mathrm{H}_{2^{\prime \prime}}\right), 1.12-1.23\left(\mathrm{~m}, 1 \mathrm{H}, \mathrm{H}_{1^{\prime \prime}}\right), 1.29-1.43(\mathrm{~m}, 4 \mathrm{H}, 2$ $\left.\times\left(\mathrm{CH}_{3}\right)_{2} \mathrm{CHCH}_{2}\right), 1.44-1.55\left(\mathrm{~m}, 1 \mathrm{H}, \mathrm{H}_{1^{\prime \prime}}\right), 1.84-1.97\left(\mathrm{~m}, 3 \mathrm{H}, \mathrm{H}_{5}\right.$, $\left.2 \times\left(\mathrm{CH}_{3}\right)_{2} \mathrm{CHCH}_{2}\right), 2.95($ br s, $1 \mathrm{H}, \mathrm{OH}), 3.17(\mathrm{td}, J=9.4,3.0 \mathrm{~Hz}$, $\left.1 \mathrm{H}, \mathrm{H}_{6}\right), 3.46\left(\mathrm{t}, J=5.6 \mathrm{~Hz}, 2 \mathrm{H}, \mathrm{NCH}_{2} \mathrm{CH}_{2} \mathrm{OH}\right), 3.74(\mathrm{t}, J=4.9 \mathrm{~Hz}$, $\left.2 \mathrm{H}, \mathrm{NCH}_{2} \mathrm{CH}_{2} \mathrm{OH}\right), 4.39-4.44\left(\mathrm{~m}, 1 \mathrm{H}, \mathrm{H}_{2}\right), 6.16\left(\right.$ br s, $\left.1 \mathrm{H}, \mathrm{H}_{4}\right)$, $6.24(\mathrm{t}, J=5.2 \mathrm{~Hz}, 1 \mathrm{H}, \mathrm{NH}) ;{ }^{13} \mathrm{C}$ NMR $\left(125 \mathrm{MHz}, \mathrm{CDCl}_{3}\right) \delta 10.4$ $\left(\mathrm{q}, \mathrm{C}_{2^{\prime \prime}}\right), 21.0\left(\mathrm{q},\left(\underline{\mathrm{CH}}_{3}\right)_{2} \mathrm{CHCH}_{2}\right), 21.4\left(\mathrm{q},\left(\mathrm{CH}_{3}\right)_{2} \mathrm{CHCH}_{2}\right), 23.4(\mathrm{t}$, $\left.\mathrm{C}_{1^{\prime \prime}}\right), 23.9\left(\mathrm{q}, 2 \mathrm{C}, 2 \times\left(\mathrm{CH}_{3}\right)_{2} \mathrm{CHCH}_{2}\right), 24.4\left(\mathrm{~d},\left(\mathrm{CH}_{3}\right)_{2} \mathrm{CHCH}_{2}\right)$, $24.6\left(\mathrm{~d},\left(\mathrm{CH}_{3}\right)_{2} \mathrm{CHCH}_{2}\right), 41.5\left(\mathrm{~d}, \mathrm{C}_{5}\right), 42.0\left(\mathrm{t},\left(\mathrm{CH}_{3}\right)_{2} \mathrm{CHCH}_{2}\right), 42.2$ $\left(\mathrm{t}, \quad\left(\mathrm{CH}_{3}\right)_{2} \mathrm{CH}_{\mathrm{CH}}\right), 42.3\left(\mathrm{t}, \quad \mathrm{NCH}_{2} \mathrm{CH}_{2} \mathrm{OH}\right), 62.1$ (t, $\mathrm{NCH}_{2} \mathrm{CH}_{2} \mathrm{OH}$ ), 72.7 (d, $\mathrm{C}_{2}$ ), $75.1\left(\mathrm{~d}, \mathrm{C}_{6}\right), 132.6$ (d, $\left.\mathrm{C}_{4}\right), 139.7$ (s, $\left.\mathrm{C}_{3}\right), 170.3\left(\mathrm{~s}, \mathrm{C}_{3} \underline{\mathrm{C}}(\mathrm{O}) \mathrm{N}\right) ; \mathrm{MS}(\mathrm{EI}) \mathrm{m} / z$ (relative intensity) $312(\mathrm{M}+$ $1)^{+}(8), 311(\mathrm{M})^{+}(23), 294(\mathrm{M}-\mathrm{OH})^{+}(5), 282(\mathrm{M}-\mathrm{Et})^{+}(7)$, $266\left(\mathrm{M}-\mathrm{CH}_{2} \mathrm{CH}_{2} \mathrm{OH}\right)^{+}(4), 254(\mathrm{M}-i-\mathrm{Bu})^{+}(28), 236(\mathrm{M}-i-\mathrm{Bu}$ $\left.-\mathrm{H}_{2} \mathrm{O}\right)^{+}(8), 225(\mathrm{M}-\mathrm{Et}-i-\mathrm{Bu})^{+}(30), 197(\mathrm{M}-2 i-\mathrm{Bu})^{+}(2)$,
$182\left(\mathrm{M}+1-i-\mathrm{Bu}-\mathrm{Me}-\mathrm{NHCH}_{2} \mathrm{CH}_{2} \mathrm{OH}\right)^{+}(100), 168(\mathrm{M}-2 i-$ $\mathrm{Bu}-\mathrm{Et})^{+}$(5); HRMS calcd for $\mathrm{C}_{18} \mathrm{H}_{33} \mathrm{NO}_{3}\left[(\mathrm{M})^{+}\right] 311.2460$, found 311.2445 .

$\left(2 S^{*}, 3 R^{*}, 4 S^{*}, 5 S^{*}, 6 R^{*}\right)$-5-Ethyl-4-hydroxy- $N$-(2-hydroxyethyl)-2,6-diisobutyltetrahydro-2H-pyran-3-carboxamide (15). To a solution of bicycle $5 \mathrm{a}(162 \mathrm{mg}, 0.46 \mathrm{mmol})$ in $\mathrm{MeOH}(3.3 \mathrm{~mL}, 0.14$ M) was added $\mathrm{MeSO}_{3} \mathrm{H}(0.02 \mathrm{~mL}, 0.32 \mathrm{mmol}, 0.7$ equiv), and the mixture was heated at $60^{\circ} \mathrm{C}$ for $8 \mathrm{~h}$. After that, it was allowed to warm to $\mathrm{rt}$ and $\mathrm{Ba}(\mathrm{OH})_{2} \cdot 8 \mathrm{H}_{2} \mathrm{O}(432 \mathrm{mg}, 1.37 \mathrm{mmol}, 3$ equiv) was added. Then, the mixture was heated again at $60{ }^{\circ} \mathrm{C}$ for an extra $2 \mathrm{~h}$ and then cooled to rt. A $1 \mathrm{M}$ aqueous solution of $\mathrm{HCl}(5 \mathrm{~mL})$ was added; the aqueous layer was extracted with $\mathrm{Et}_{2} \mathrm{O}(3 \times 5 \mathrm{~mL})$, and the combined organic layers were washed with brine $(15 \mathrm{~mL})$, dried over $\mathrm{MgSO}_{4}$, filtered, concentrated, and purified by flash chromatography $(21 \mathrm{~cm}$ of height of silica gel, EtOAc) to yield title compound 15 (89 $\mathrm{mg}, 60 \%)$ as an amorphous white solid: $R_{f}=0.33$ (DCM/MeOH 90:10), 0.51 (EtOAc/HOAc 95:5); ${ }^{1} \mathrm{H}$ NMR (500 MHz, $\left.\mathrm{CDCl}_{3}\right) \delta 0.85-0.93(\mathrm{~m}$, $15 \mathrm{H}, 5 \times \mathrm{Me}), 1.10-1.16\left(\mathrm{~m}, 1 \mathrm{H},\left(\mathrm{CH}_{3}\right)_{2} \mathrm{CHCH}_{2}\right), 1.21-1.27(\mathrm{~m}$, $\left.2 \mathrm{H}, \mathrm{H}_{5},\left(\mathrm{CH}_{3}\right)_{2} \mathrm{CHCH}_{2}\right), 1.29-1.39\left(\mathrm{~m}, 1 \mathrm{H},\left(\mathrm{CH}_{3}\right)_{2} \mathrm{CHCH}_{2}\right), 1.37-$ $1.46\left(\mathrm{~m}, 1 \mathrm{H},\left(\mathrm{CH}_{3}\right)_{2} \mathrm{CHCH}_{2}\right), 1.49-1.56\left(\mathrm{~m}, 1 \mathrm{H}, \mathrm{H}_{1^{\prime \prime}}\right), 1.61-1.70$ $\left(\mathrm{m}, 1 \mathrm{H}, \mathrm{H}_{1^{\prime \prime}}\right), 1.83-1.93\left(\mathrm{~m}, 2 \mathrm{H}, 2 \times\left(\mathrm{CH}_{3}\right)_{2} \mathrm{CHCH}_{2}\right), 1.98(\mathrm{dd}, J=$ $\left.9.6 \mathrm{~Hz}, 1 \mathrm{H}, \mathrm{H}_{3}\right), 3.15-3.22\left(\mathrm{~m}, 1 \mathrm{H}, \mathrm{NCH}_{2} \mathrm{CH}_{2} \mathrm{OH}\right), 3.27$ (td, $J=$ $\left.10.2,1.9 \mathrm{~Hz}, 1 \mathrm{H}, \mathrm{H}_{6}\right), 3.40-3.56(\mathrm{~m}, 2 \mathrm{H}, 2 \times \mathrm{OH}), 3.52(\mathrm{td}, J=10.1$, $\left.1.8 \mathrm{~Hz}, 1 \mathrm{H}, \mathrm{H}_{2}\right), 3.60-3.74\left(\mathrm{~m}, 2 \mathrm{H}, 1 \times \mathrm{NCH}_{2} \mathrm{CH}_{2} \mathrm{OH}, 1 \times\right.$ $\mathrm{NCH}_{2} \mathrm{CH}_{2} \mathrm{OH}$ ), 3.76-3.82(m, $\left.1 \times \mathrm{NCH}_{2} \mathrm{CH}_{2} \mathrm{OH}\right), 3.89$ (dd, $J=$ $\left.10.1,10.1 \mathrm{~Hz}, 1 \mathrm{H}, \mathrm{H}_{4}\right), 6.29($ br s, $1 \mathrm{H}, \mathrm{NH}) ;{ }^{13} \mathrm{C}$ NMR $(125 \mathrm{MHz}$, $\left.\mathrm{CDCl}_{3}\right) \delta 9.5\left(\mathrm{q}, \mathrm{C}_{2^{\prime \prime}}\right), 19.0\left(\mathrm{t}, \mathrm{C}_{1^{\prime \prime}}\right), 21.1\left(\mathrm{q}, 2 \times\left(\mathrm{CH}_{3}\right)_{2} \mathrm{CHCH}_{2}\right)$, $23.9\left(\mathrm{q},\left(\mathrm{CH}_{3}\right)_{2} \mathrm{CHCH}_{2}\right), 24.1\left(\mathrm{q},\left(\mathrm{CH}_{3}\right)_{2} \mathrm{CHCH}_{2}\right), 24.2$ (d, $\left.\left.\left(\mathrm{CH}_{3}\right)_{2} \mathrm{CHCH}\right)_{2}\right), 24.3\left(\mathrm{~d},\left(\mathrm{CH}_{3}\right)_{2} \mathrm{CHCH}_{2}\right), 42.1\left(\mathrm{t}, \mathrm{NCH}_{2} \mathrm{CH}_{2} \mathrm{OH}\right)$, $42.3\left(\mathrm{t},\left(\mathrm{CH}_{3}\right)_{2} \mathrm{CHCH}_{2}\right), 42.9\left(\mathrm{t},\left(\mathrm{CH}_{3}\right)_{2} \mathrm{CHCH}_{2}\right), 48.3\left(\mathrm{~d}, \mathrm{C}_{5}\right), 59.5$ $\left(\mathrm{d}, \mathrm{C}_{3}\right), 61.4\left(\mathrm{t}, \mathrm{NCH}_{2} \mathrm{CH}_{2} \mathrm{OH}\right), 71.7\left(\mathrm{~d}, \mathrm{C}_{4}\right), 74.7\left(\mathrm{~d}, \mathrm{C}_{2}\right), 76.0(\mathrm{~d}$, $\left.\mathrm{C}_{6}\right), 174.1\left(\mathrm{~s}, \mathrm{C}_{3} \underline{\mathrm{CONH}}\right) ; \mathrm{MS}(\mathrm{EI}) \mathrm{m} / z$ (relative intensity) $330(\mathrm{M}+$ $2)^{+}(1), 328(\mathrm{M})^{+}(1), 312(\mathrm{M}-\mathrm{OH})^{+}(1), 311\left(\mathrm{M}-\mathrm{H}_{2} \mathrm{O}\right)^{+}(2)$, $298\left(\mathrm{M}-\mathrm{CH}_{2} \mathrm{OH}\right)^{+}(1), 272(\mathrm{M}-i-\mathrm{Bu})^{+}(8), 227(\mathrm{M}-i-\mathrm{Bu}-$ $\left.\mathrm{CH}_{2} \mathrm{CH}_{2} \mathrm{OH}\right)^{+}(2), 216(\mathrm{M}+1-2 i-\mathrm{Bu})^{+}(100), 188(\mathrm{M}+2-2 i-\mathrm{Bu}$ $-\mathrm{Et})^{+}(16)$; HRMS calcd for $\mathrm{C}_{18} \mathrm{H}_{34} \mathrm{NO}_{4}\left[(\mathrm{M})^{+}\right] 328.2488$, found 328.2497.

$\left(2 S^{*}, 3 S^{*}, 4 S^{*}, 5 R^{*}, 6 R^{*}\right)-5$-Ethyl-4-\{[(2-hydroxyethyl)carbamoyl] oxy\}-2,6-diisobutyltetrahydro- $2 \mathrm{H}$-pyran-3-carboxylic Acid (16). To an ice-cooled solution of bicycle 5a (1.19 g, 3.36 $\mathrm{mmol})$ in a $3: 1 \mathrm{THF} / \mathrm{H}_{2} \mathrm{O}$ mixture $(60 \mathrm{~mL}, 0.05 \mathrm{M})$ was added a $35 \%$ $(\mathrm{w} / \mathrm{w})$ aqueous solution of $\mathrm{H}_{2} \mathrm{O}_{2}(1.8 \mathrm{~mL}, 20.2 \mathrm{mmol}, 6$ equiv) and $\mathrm{LiOH} \cdot \mathrm{H}_{2} \mathrm{O}$ ( $287 \mathrm{mg}, 6.72 \mathrm{mmol}, 2$ equiv). The mixture was allowed to warm to rt and stirred for $21 \mathrm{~h}$, and then an aliquot was taken, diluted with a small amount of EtOAc, and treated with a few drops of a $5 \% \mathrm{HCl}$ aqueous solution. TLC analysis of the treated aliquot revealed full conversion of the starting material. After that, the reaction mixture was cooled to $0{ }^{\circ} \mathrm{C}$ and the reaction was quenched with a $1.5 \mathrm{M}$ aqueous solution of $\mathrm{Na}_{2} \mathrm{SO}_{3}(60 \mathrm{~mL})$. Then, the THF was evaporated in the rotavap, and the remaining solution was diluted with $\mathrm{H}_{2} \mathrm{O}(40 \mathrm{~mL})$, washed with $\mathrm{DCM}(100 \mathrm{~mL})$, acidified to $\mathrm{pH} 1$ with a $5 \% \mathrm{HCl}$ aqueous solution, and extracted with EtOAc $(3 \times 150$ $\mathrm{mL})$. The combined organic layers were washed with brine $(500 \mathrm{~mL})$, dried over $\mathrm{MgSO}_{4}$, filtered, and concentrated. One-tenth of the crude was separated and purified by flash chromatography $(9 \mathrm{~cm}$ of height of silica gel, $100 \mathrm{~mL}$ of EtOAc and then EtOAc/MeOH 80:20) to yield title compound 16 (98 mg, which mathematically means a total yield of $79 \%$ ) as an amorphous white solid. The nonpurified crude was consumed in the synthesis of THP 17: $R_{f}=0.24$ (EtOAc), 0.38 (EtOAc/HOAc 95:5), 0.39 (EtOAc/MeOH 90:10), 0.54 (EtOAc/ $\mathrm{MeOH}$ 80:20), 0.75 (DCM/MeOH 80:20); ${ }^{1} \mathrm{H}$ NMR (500 MHz, $\left.\mathrm{CDCl}_{3}\right) \delta 0.85-0.93\left(\mathrm{~m}, 15 \mathrm{H}, 5 \times \mathrm{CH}_{3}\right), 1.16-1.25(\mathrm{~m}, 1 \mathrm{H}, 1 \times$ $\left.\left(\mathrm{CH}_{3}\right)_{2} \mathrm{CHC}_{2}\right), 1.26-1.35\left(\mathrm{~m}, 1 \mathrm{H}, 1 \times\left(\mathrm{CH}_{3}\right)_{2} \mathrm{CHCH}_{2}\right), 1.37-$ $1.55\left(\mathrm{~m}, 5 \mathrm{H}, \mathrm{H}_{5}, 1 \times \mathrm{H}_{1^{\prime}}, 2 \times \mathrm{H}_{1^{\prime \prime}}, 1 \times \mathrm{H}_{1^{\prime \prime \prime}}\right), 1.83-1.95(\mathrm{~m}, 2 \mathrm{H}, 2 \times$ $\left.\left(\mathrm{CH}_{3}\right)_{2} \mathrm{CHCH}_{2}\right), 2.35\left(\mathrm{dd}, J=10.2,10.2 \mathrm{~Hz}, 1 \mathrm{H}, \mathrm{H}_{3}\right), 3.08-3.16(\mathrm{~m}$, $\left.1 \mathrm{H}, \mathrm{NC}_{2} \mathrm{CH}_{2} \mathrm{OH}\right), 3.30-3.36\left(\mathrm{~m}, 1 \mathrm{H}, \mathrm{H}_{6}\right), 3.43-3.67\left(\mathrm{~m}, 3 \mathrm{H}, \mathrm{H}_{2}\right.$, $\left.1 \times \mathrm{NCH}_{2} \mathrm{CH}_{2} \mathrm{OH}, 1 \times \mathrm{NCH}_{2} \mathrm{CH}_{2} \mathrm{OH}\right), 3.72-3.82(\mathrm{~m}, 1 \mathrm{H}$, $\mathrm{NCH}_{2} \mathrm{CH}_{2} \mathrm{OH}$ ), 5.15 (dd, $\left.J=10.5,10.5 \mathrm{~Hz}, 1 \mathrm{H}, \mathrm{H}_{4}\right), 5.23-5.32$ $(\mathrm{m}, 1 \mathrm{H}, \mathrm{NH}) ;{ }^{1} \mathrm{H}$ NMR $\left(500 \mathrm{MHz},\left(\mathrm{D}_{3} \mathrm{C}\right)_{2} \mathrm{CO}\right) \delta 0.85-0.94(\mathrm{~m}$, $\left.15 \mathrm{H}, 5 \times \mathrm{CH}_{3}\right), 1.13-1.21\left(\mathrm{~m}, 1 \mathrm{H}, \mathrm{H}_{1^{\prime}}\right), 1.29-1.34\left(\mathrm{~m}, 1 \mathrm{H}, \mathrm{H}_{5}\right)$, 
$1.35-1.40\left(\mathrm{~m}, 2 \mathrm{H}, \mathrm{H}_{1^{\prime \prime}}\right), 1.42-1.52\left(\mathrm{~m}, 3 \mathrm{H}, 1 \times \mathrm{H}_{1^{\prime}}, 2 \times \mathrm{H}_{1^{\prime \prime}}\right), 1.85-$ $1.97\left(\mathrm{~m}, 2 \mathrm{H}, 2 \times\left(\mathrm{CH}_{3}\right)_{2} \mathrm{CHCH}_{2}\right), 2.25(\mathrm{dd}, J=10.1,10.1 \mathrm{~Hz}, 1 \mathrm{H}$, $\left.\mathrm{H}_{3}\right), 3.17-3.24\left(\mathrm{~m}, 2 \mathrm{H}, \mathrm{NC} \underline{\mathrm{H}}_{2} \mathrm{CH}_{2} \mathrm{OH}\right), 3.39(\mathrm{td}, J=9.7,4.0 \mathrm{~Hz}$, $\left.1 \mathrm{H}, \mathrm{H}_{6}\right), 3.50-3.60\left(\mathrm{~m}, 3 \mathrm{H}, \mathrm{H}_{2}, \mathrm{NCH}_{2} \mathrm{CH}_{2} \mathrm{OH}\right), 5.16$ (dd, $J=10.5$, $\left.10.5 \mathrm{~Hz}, 1 \mathrm{H}, \mathrm{H}_{4}\right), 6.19($ br s, $1 \mathrm{H}, \mathrm{NH}) ;{ }^{13} \mathrm{C} \mathrm{NMR}(125 \mathrm{MHz}$, $\left.\mathrm{CDCl}_{3}\right) \delta 9.7\left(\mathrm{q}, \mathrm{C}_{2^{\prime \prime}}\right), 19.4\left(\mathrm{t}, \mathrm{C}_{1^{\prime \prime}}\right), 21.3(\mathrm{q}, 2 \mathrm{C}, 2 \times$ $\left.\left(\mathrm{CH}_{3}\right)_{2} \mathrm{CHCH}_{2}\right), 23.8 \quad\left(\mathrm{q}, \quad\left(\mathrm{CH}_{3}\right)_{2} \mathrm{CHCH}_{2}\right), 24.1 \quad(\mathrm{q}$, $\left.\left(\mathrm{C}_{3}\right)_{2} \mathrm{CHCH}_{2}\right), 24.4\left(\mathrm{~d},\left(\mathrm{CH}_{3}\right)_{2} \underline{\mathrm{C}} \mathrm{HCH}_{2}\right), 24.5$ (d, $\left.\left(\mathrm{CH}_{3}\right)_{2} \underline{\mathrm{C}} \mathrm{HCH}_{2}\right), 42.1\left(\mathrm{t},\left(\mathrm{CH}_{3}\right)_{2} \mathrm{CHCH}_{2}\right), 43.2\left(\mathrm{t},\left(\mathrm{CH}_{3}\right)_{2} \mathrm{CHCH}_{2}\right)$, $43.6\left(\mathrm{t}, \mathrm{NCH}_{2} \mathrm{CH}_{2} \mathrm{OH}\right), 46.4\left(\mathrm{~d}, \mathrm{C}_{5}\right), 56.2\left(\mathrm{~d}, \mathrm{C}_{3}\right), 61.7(\mathrm{t}$, $\left.\mathrm{NCH}_{2} \mathrm{CH}_{2} \mathrm{OH}\right), 75.1\left(\mathrm{~d}, \mathrm{C}_{4}\right), 76.4\left(\mathrm{~d}, \mathrm{C}_{6}\right), 76.6\left(\mathrm{~d}, \mathrm{C}_{2}\right), 157.7$ (s, $\mathrm{OC}(\mathrm{O}) \mathrm{NH}), 175.5\left(\mathrm{~s}, \mathrm{CO}_{2} \mathrm{H}\right) ;{ }^{13} \mathrm{C}$ NMR $\left(125 \mathrm{MHz},\left(\mathrm{D}_{3} \mathrm{C}\right)_{2} \mathrm{CO}\right) \delta$ $9.5\left(\mathrm{q}, \mathrm{C}_{2^{\prime \prime}}\right), 19.5\left(\mathrm{t}, \mathrm{C}_{1^{\prime \prime}}\right), 21.4\left(\mathrm{q},\left(\mathrm{CH}_{3}\right)_{2} \mathrm{CHCH}_{2}\right), 21.5(\mathrm{q}$, $\left.\left(\mathrm{CH}_{3}\right)_{2} \mathrm{CHCH}_{2}\right), 24.1 \quad\left(\mathrm{q}, \quad\left(\mathrm{C}_{3}\right)_{2} \mathrm{CHCH}_{2}\right), 24.3$ (q, $\left.\left(\mathrm{C}_{3}\right)_{2} \mathrm{CHCH}_{2}\right), 24.90\left(\mathrm{~d}, \quad\left(\mathrm{CH}_{3}\right)_{2} \mathrm{CHCH}_{2}\right), 25.01$ (d, $\left.\left(\mathrm{CH}_{3}\right)_{2} \underline{\mathrm{C}} \mathrm{HCH}_{2}\right), 42.7\left(\mathrm{t}, \mathrm{C}_{1^{\prime \prime}}\right), 43.9\left(\mathrm{t}, \mathrm{C}_{1^{\prime}}\right), 44.3\left(\mathrm{t}, \mathrm{NCH}_{2} \mathrm{CH}_{2} \mathrm{OH}\right)$, $47.5\left(\mathrm{~d}, \mathrm{C}_{5}\right), 56.5\left(\mathrm{~d}, \mathrm{C}_{3}\right), 61.9\left(\mathrm{t}, \mathrm{NCH}_{2} \underline{\mathrm{CH}}_{2} \mathrm{OH}\right), 74.0\left(\mathrm{~d}, \mathrm{C}_{4}\right), 75.3$ $\left(\mathrm{d}, \mathrm{C}_{2}\right), 76.4\left(\mathrm{~d}, \mathrm{C}_{6}\right), 157.2(\mathrm{~s}, \mathrm{OC}(\mathrm{O}) \mathrm{NH}), 173.2\left(\mathrm{~s}, \mathrm{CO}_{2} \mathrm{H}\right) ; \mathrm{MS}$ (EI) $\mathrm{m} / z$ (relative intensity) $344(\mathrm{M}-\mathrm{Et})^{+}(1), 343(\mathrm{M}-1-\mathrm{Et})^{+}$ (4), $316(\mathrm{M}-i-\mathrm{Bu})^{+}(4), 287(\mathrm{M}-i-\mathrm{Bu}-\mathrm{Et})^{+}(4), 269(\mathrm{M}-$

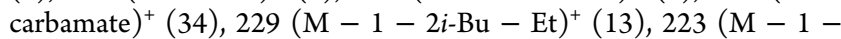
carbamate $\left.-\mathrm{CO}_{2} \mathrm{H}\right)^{+}(20), 211(\mathrm{M}-1-i-\mathrm{Bu}-\text { carbamate })^{+}(100)$, $155(\mathrm{M}-\text { carbamate }-2 i-\mathrm{Bu})^{+}(27), 126(\mathrm{M}-2 i-\mathrm{Bu}$ - carbamate $2 \mathrm{Et})^{+}(28), 110\left(\mathrm{M}-2 i-\mathrm{Bu}-\text { carbamate }-\mathrm{CO}_{2} \mathrm{H}\right)^{+}(17)$; HRMS calcd for $\mathrm{C}_{17} \mathrm{H}_{29} \mathrm{NO}_{6}\left[(\mathrm{M}-1-\mathrm{Et})^{+}\right] 343.1995$, found 343.1987; HRMS calcd for $\mathrm{C}_{12} \mathrm{H}_{19} \mathrm{O}_{3}\left[(\mathrm{M}+1-i \text {-Bu - carbamate })^{+}\right] 211.1334$, found 211.1339 .

$\left(2 S^{*}, 3 R^{*}, 4 S^{*}, 5 S^{*}, 6 R^{*}\right)$-5-Ethyl-4-hydroxy-2,6-diisobutyltetrahydro-2H-pyran-3-carboxylic Acid (17). THP 16 (95 mg, 0.26 $\mathrm{mmol}$ ) was dissolved in a $3: 1: 1 \mathrm{THF} / \mathrm{MeOH} / \mathrm{H}_{2} \mathrm{O}$ mixture $(3.5 \mathrm{~mL}$, $0.07 \mathrm{M})$, and $\mathrm{LiOH} \cdot \mathrm{H}_{2} \mathrm{O}(164 \mathrm{mg}, 3.83 \mathrm{mmol}, 15$ equiv) was added. The reaction mixture was heated at $80{ }^{\circ} \mathrm{C}$ for $24 \mathrm{~h}$. After that, an aliquot was taken, diluted with a small amount of EtOAc, and treated with a few drops of a $5 \% \mathrm{HCl}$ aqueous solution. TLC analysis of the treated aliquot revealed full conversion of the starting material. The organic solvents were removed in the rotavap, and then the aqueous mixture was extracted with EtOAc $(3 \times 5 \mathrm{~mL})$. The combined organic layers were washed with brine $(15 \mathrm{~mL})$, and when the huge amount of emulsion disappeared, the organic layer was separated, dried over $\mathrm{MgSO}_{4}$, filtered, concentrated, and purified by flash chromatography (14 cm of height of silica gel, $60 \mathrm{~mL}$ of a $98: 2$ $\mathrm{EtOAc} / \mathrm{MeOH}$ mixture followed by $60 \mathrm{~mL}$ of a $90: 10 \mathrm{EtOAc} / \mathrm{MeOH}$ mixture and $60 \mathrm{~mL}$ of an 80:20 EtOAc/MeOH mixture) to yield title compound 17 (52 mg, 70\%) as an amorphous white solid. A similar yield was obtained when the reaction was performed with nonpurified THP 16 as the starting material. THP 17 shows decreasing solubility in deuterated solvents according to the order DMSO- $d_{6} \gg$ acetone- $d_{6}$ $>\mathrm{MeOD} \gg \mathrm{CDCl}_{3}>\mathrm{C}_{6} \mathrm{D}_{6} \gg \mathrm{D}_{2} \mathrm{O}$ (totally insoluble): $R_{f}=0.28$ (EtOAc), 0.46 (EtOAc/MeOH 95:5), 0.53 (EtOAc/HOAc 97.5:2.5), 0.69 (EtOAc/HOAc 95:5); ${ }^{1} \mathrm{H}$ NMR $\left(500 \mathrm{MHz},\left(\mathrm{D}_{3} \mathrm{C}\right)_{2} \mathrm{CO}\right) \delta$ $0.84-0.93\left(\mathrm{~m}, 15 \mathrm{H}, 5 \times \mathrm{CH}_{3}\right), 1.12-1.21\left(\mathrm{~m}, 2 \mathrm{H}, \mathrm{H}_{5}, 1 \times\right.$ $\left.\left(\mathrm{CH}_{3}\right)_{2} \mathrm{CHCH}_{2}\right), 1.34-1.39$ (m, $\left.2 \mathrm{H},\left(\mathrm{CH}_{3}\right)_{2} \mathrm{CHCH}_{2}\right), 1.44-1.49$ $\left(\mathrm{m}, 1 \mathrm{H},\left(\mathrm{CH}_{3}\right)_{2} \mathrm{CHCH}_{2}\right), 1.49-1.56\left(\mathrm{~m}, 1 \mathrm{H}, \mathrm{H}_{1^{\prime \prime}}\right), 1.69-1.79(\mathrm{~m}$, $\left.1 \mathrm{H}, \mathrm{H}_{1^{\prime \prime}}\right), 1.84-1.96\left(\mathrm{~m}, 2 \mathrm{H},\left(\mathrm{CH}_{3}\right)_{2} \mathrm{CHCH}_{2}\right), 2.17(\mathrm{dd}, J=10.0$, $\left.10.0 \mathrm{~Hz}, 1 \mathrm{H}, \mathrm{H}_{3}\right), 3.25-3.31\left(\mathrm{~m}, 1 \mathrm{H}, \mathrm{H}_{6}\right), 3.42(\mathrm{td}, J=10.2,2.0 \mathrm{~Hz}$, $\left.1 \mathrm{H}, \mathrm{H}_{2}\right), 3.79\left(\mathrm{dd}, J=10.2,10.2 \mathrm{~Hz}, 1 \mathrm{H}, \mathrm{H}_{4}\right) ;{ }^{13} \mathrm{C} \mathrm{NMR}(125 \mathrm{MHz}$, $\left.\left(\mathrm{D}_{3} \mathrm{C}\right)_{2} \mathrm{CO}\right) \delta 9.8\left(\mathrm{q}, \mathrm{C}_{2^{\prime \prime}}\right), 19.4\left(\mathrm{t}, \mathrm{C}_{1^{\prime \prime}}\right), 21.4\left(\mathrm{q},\left(\mathrm{CH}_{3}\right)_{2} \mathrm{CHCH}_{2}\right)$, $21.5\left(\mathrm{q}, \quad\left(\mathrm{CH}_{3}\right)_{2} \mathrm{CHCH}_{2}\right), 24.2\left(\mathrm{q},\left(\mathrm{CH}_{3}\right)_{2} \mathrm{CHCH}_{2}\right), 24.4(\mathrm{q}$, $\left.\left(\mathrm{CH}_{3}\right)_{2} \mathrm{CHCH}_{2}\right), 24.92\left(\mathrm{~d}, \quad\left(\mathrm{CH}_{3}\right)_{2} \mathrm{CHCH}_{2}\right), 24.94$ (d, $\left.\left(\mathrm{CH}_{3}\right)_{2} \underline{\mathrm{C}} \mathrm{HCH}_{2}\right), 43.0\left(\mathrm{t},\left(\mathrm{CH}_{3}\right)_{2} \mathrm{CHCH}_{2}\right), 44.1\left(\mathrm{t},\left(\mathrm{CH}_{3}\right)_{2} \mathrm{CHCH}_{2}\right)$, $49.4\left(\mathrm{~d}, \mathrm{C}_{5}\right), 59.1\left(\mathrm{~d}, \mathrm{C}_{3}\right), 72.4\left(\mathrm{~d}, \mathrm{C}_{4}\right), 75.5\left(\mathrm{~d}, \mathrm{C}_{2}\right), 76.7\left(\mathrm{~d}, \mathrm{C}_{6}\right)$, $174.7\left(\mathrm{~s}, \mathrm{CO}_{2} \mathrm{H}\right) ; \mathrm{MS}(\mathrm{EI}) \mathrm{m} / z$ (relative intensity) $268\left(\mathrm{M}-\mathrm{H}_{2} \mathrm{O}\right)^{+}$ (18), $240\left(\mathrm{M}-1-\mathrm{CO}_{2} \mathrm{H}\right)^{+}(2), 229(\mathrm{M}-i-\mathrm{Bu})^{+}(49), 211(\mathrm{M}-i-$ $\left.\mathrm{Bu}-\mathrm{H}_{2} \mathrm{O}\right)^{+}(28), 182\left(\mathrm{M}-2-i-\mathrm{Bu}-\mathrm{CO}_{2} \mathrm{H}\right)^{+}(13), 173(\mathrm{M}+1$ $-2 i-\mathrm{Bu})^{+}(17)$; HRMS calcd for $\mathrm{C}_{16} \mathrm{H}_{28} \mathrm{O}_{3}\left[\left(\mathrm{M}-\mathrm{H}_{2} \mathrm{O}\right)^{+}\right]$268.2038, found 268.2034 .

$\left(2 R^{*}, 3 S^{*}, 4 S^{*}, 5 R^{*}, 6 S^{*}\right)-3$-Ethyl-5-\{[(2-hydroxyethyl)(methyl)amino]methyl\}-2,6-diisobutyltetrahydro-2 $\mathrm{H}$-pyran-4-ol (18). To an ice-cooled $1 \mathrm{M}$ solution of DIBAL-H in hexanes $(4 \mathrm{~mL}, 4$ mmol, 9 equiv) was added dropwise, under an Ar atmosphere and for
$7 \mathrm{~min}$, a solution of bicycle $5 \mathrm{a}(159 \mathrm{mg}, 0.45 \mathrm{mmol})$ in $\mathrm{Et}_{2} \mathrm{O}(4.5 \mathrm{~mL}$, $0.1 \mathrm{M})$. Five minutes after the addition, TLC analysis revealed that the reaction was completed. At $20 \mathrm{~min}$, the mixture was diluted with $\mathrm{Et}_{2} \mathrm{O}(20 \mathrm{~mL})$ and a saturated Rochelle salt aqueous solution $(5 \mathrm{~mL})$ was added. The mixture was vigorously stirred for $1 \mathrm{~h}$, until two clear phases were observed when the stirring was stopped. The layers were separated in a separatory funnel; the aqueous layer was extracted with $\mathrm{Et}_{2} \mathrm{O}(3 \times 20 \mathrm{~mL})$, and the combined organic layers were dried over $\mathrm{MgSO}_{4}$, filtered, concentrated, and purified by flash chromatography (16 cm of height of silica gel, $\mathrm{DCM} / \mathrm{MeOH} 95: 5)$ to yield title compound $18(97 \mathrm{mg}, 66 \%)$ as a yellowish oil: $R_{f}=0.24(\mathrm{DCM} /$ $\mathrm{MeOH} 95: 5) ;{ }^{1} \mathrm{H}$ NMR $\left(500 \mathrm{MHz}, \mathrm{CDCl}_{3}\right) \delta 0.79-0.90(\mathrm{~m}, 15 \mathrm{H}, 5$ $\left.\times\left(\mathrm{CH}_{3}\right)\right), 1.15-1.20\left(\mathrm{~m}, 1 \mathrm{H}, \mathrm{H}_{1^{\prime \prime}}\right), 1.25-1.32\left(\mathrm{~m}, 2 \mathrm{H}, \mathrm{H}_{3}, \mathrm{H}_{1^{\prime}}\right)$, 1.35-1.42 (m, $\left.2 \mathrm{H}, \mathrm{H}_{1^{\prime}}, \mathrm{H}_{1^{\prime \prime}}\right), 1.43-1.49\left(\mathrm{~m}, 1 \mathrm{H}, \mathrm{H}_{1^{\prime \prime}}\right), 1.52-1.60$ (m, $\left.1 \mathrm{H}, \mathrm{H}_{5}\right), 1.58-1.65\left(\mathrm{~m}, 1 \mathrm{H}, \mathrm{H}_{1^{\prime \prime}}\right), 1.80-1.92\left(\mathrm{~m}, 2 \mathrm{H}, \mathrm{H}_{2^{\prime}}, \mathrm{H}_{2^{\prime \prime}}\right)$, $2.31\left(\mathrm{~s}, 3 \mathrm{H}, \mathrm{CH}_{3} \mathrm{~N}\right), 2.40-2.47\left(\mathrm{~m}, 2 \mathrm{H}, 1 \times \mathrm{C}_{5} \mathrm{CH}_{2} \mathrm{~N}, 1 \times\right.$ $\left.\mathrm{NC}_{2} \mathrm{CH}_{2} \mathrm{OH}\right), 2.47$ (dd, $\left.J=12.6,2.9 \mathrm{~Hz}, 1 \mathrm{H}, \mathrm{C}_{5} \mathrm{CH}_{2} \mathrm{~N}\right), 2.67-2.72$ $\left(\mathrm{m}, 1 \mathrm{H}, \mathrm{NCH}_{2} \mathrm{CH}_{2} \mathrm{OH}\right), 2.97\left(\mathrm{td}, J=9.5,1.8 \mathrm{~Hz}, 1 \mathrm{H}, \mathrm{H}_{6}\right), 3.17$ (td, $J$ $\left.=10.4,1.9 \mathrm{~Hz}, 1 \mathrm{H}, \mathrm{H}_{2}\right), 3.56\left(\mathrm{dd}, J=9.7,9.7 \mathrm{~Hz}, 1 \mathrm{H}, \mathrm{H}_{4}\right), 3.64-3.74$ $\left(\mathrm{m}, 2 \mathrm{H}, \mathrm{NCH}_{2} \mathrm{CH}_{2} \mathrm{OH}\right) ;{ }^{13} \mathrm{C}$ NMR $\left(125 \mathrm{MHz}, \mathrm{CDCl}_{3}\right) \delta 9.3(\mathrm{q}$, $\left.\mathrm{C}_{2^{\prime \prime}}\right), 18.5\left(\mathrm{t}, \mathrm{C}_{1^{\prime \prime}}\right), 21.05\left(\mathrm{q},\left(\mathrm{CH}_{3}\right)_{2} \mathrm{CHCH}_{2}\right), 21.06(\mathrm{q}$, $\left.\left(\mathrm{CH}_{3}\right)_{2} \mathrm{CHCH}_{2}\right), 24.0$ (q, $\left.\left(\mathrm{CH}_{3}\right)_{2} \mathrm{CHCH}_{2}\right), 24.1$ (d, 2C, $2 \times$ $\left.\left(\mathrm{CH}_{3}\right)_{2} \mathrm{CHCH}_{2}\right), 24.2\left(\mathrm{q},\left(\mathrm{CH}_{3}\right)_{2} \mathrm{CHCH}_{2}\right), 42.1\left(\mathrm{t}, \mathrm{C}_{1^{\prime}}\right), 42.5(\mathrm{t}$,

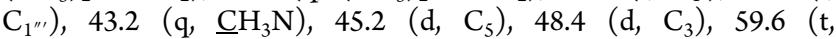
$\left.\mathrm{NCH}_{2} \mathrm{CH}_{2} \mathrm{OH}\right), 60.4\left(\mathrm{t}, \mathrm{NCH}_{2} \mathrm{CH}_{2} \mathrm{OH}\right), 61.2\left(\mathrm{t}, \mathrm{C}_{5} \mathrm{CH}_{2} \mathrm{~N}\right), 74.6$ $\left(\mathrm{d}, \mathrm{C}_{6}\right), 75.9\left(\mathrm{~d}, \mathrm{C}_{2}\right), 77.2\left(\mathrm{~d}, \mathrm{C}_{4}\right) ; \mathrm{MS}(\mathrm{EI}) \mathrm{m} / \mathrm{z}$ (relative intensity) $330(\mathrm{M}+1)^{+}(1), 329(\mathrm{M})^{+}(1), 314(\mathrm{M}-\mathrm{Me})^{+}(1), 298(\mathrm{M}-$ $\left.\mathrm{CH}_{2} \mathrm{OH}\right)^{+}(14), 272(\mathrm{M}-t-\mathrm{Bu})^{+}(1), 216(\mathrm{M}+1-2 t-\mathrm{Bu})^{+}(6)$, $186(\mathrm{M}-2 \mathrm{t}-\mathrm{Bu}-\mathrm{Et})^{+}(1), 88(100)$; HRMS calcd for $\mathrm{C}_{19} \mathrm{H}_{39} \mathrm{NO}_{3}$ $\left[(\mathrm{M})^{+}\right]$329.2930, found 329.2922.

$\left(2 R^{*}, 3 S^{*}, 4 S^{*}, 5 R^{*}, 6 S^{*}\right)$-3-Ethyl-5-(hydroxymethyl)-2,6-diisobutyltetrahydro-2H-pyran-4-ol (19). To a solution of bicycle $5 \mathrm{a}$ $(285 \mathrm{mg}, 0.73 \mathrm{mmol})$ in THF $(7 \mathrm{~mL}, 0.1 \mathrm{M})$ was added dropwise, at rt and under an Ar atmosphere, a $1 \mathrm{M}$ solution of DIBAL-H in hexanes $(8 \mathrm{~mL}, 8 \mathrm{mmol}, 11$ equiv). Then, the reaction mixture was heated at $66{ }^{\circ} \mathrm{C}$ for $19 \mathrm{~h}$. Once TLC analysis revealed that the reaction was completed, the mixture was cooled to rt and diluted with $\mathrm{Et}_{2} \mathrm{O}(50 \mathrm{~mL})$ and the reaction was quenched with a saturated Rochelle salt aqueous solution $(10 \mathrm{~mL})$. The mixture was vigorously stirred for $1 \mathrm{~h}$ and then was poured into a separatory funnel together with $\mathrm{H}_{2} \mathrm{O}(40 \mathrm{~mL})$. The layers were separated; the aqueous layer was extracted with $\mathrm{Et}_{2} \mathrm{O}(3 \times 50 \mathrm{~mL})$, and the combined organic layers were dried over $\mathrm{MgSO}_{4}$, filtered, concentrated, and purified by flash chromatography (35 cm of height of silica gel, $n$-hexane/EtOAc 75:25) to yield diol $19(71 \mathrm{mg}, 36 \%)$. Alternatively, carbamate 20 (65 $\mathrm{mg}, 0.18 \mathrm{mmol}$ ) was dissolved in a $1: 1: 1 \mathrm{THF} / \mathrm{MeOH} / \mathrm{H}_{2} \mathrm{O}$ mixture $(2.6 \mathrm{~mL}, 0.07 \mathrm{M})$, and $\mathrm{LiOH} \cdot \mathrm{H}_{2} \mathrm{O}(78 \mathrm{mg}, 1.85 \mathrm{mmol}, 10$ equiv) was added. The mixture was heated at $80{ }^{\circ} \mathrm{C}$ with a saturated $\mathrm{NH}_{4} \mathrm{Cl}$ aqueous solution $(5 \mathrm{~mL})$, and the aqueous mixture was extracted with DCM $(3 \times 10 \mathrm{~mL})$. The combined organic layers were dried over $\mathrm{MgSO}_{4}$, filtered, concentrated, and purified by flash chromatography (14 cm of height of silica gel, $n$-hexane/EtOAc 60:40) to yield diol 19 (38 mg, 80\%): amorphous white solid; $R_{f}=0.25$ ( $n$-hexane $/ \mathrm{EtOAc}$ 50:50), 0.40 (n-hexane/EtOAc 20:80); ${ }^{1} \mathrm{H}$ NMR (500 MHz, $\mathrm{CDCl}_{3}$ ) $\delta 0.84\left(\mathrm{~d}, J=6.6 \mathrm{~Hz}, 3 \mathrm{H},\left(\mathrm{CH}_{3}\right)_{2} \mathrm{CHCH}_{2}\right), 0.86(\mathrm{~d}, J=6.4 \mathrm{~Hz}, 3 \mathrm{H}$, $\left.\left(\mathrm{CH}_{3}\right)_{2} \mathrm{CHCH}_{2}\right), 0.89\left(\mathrm{t}, J=7.5 \mathrm{~Hz}, 3 \mathrm{H}, \mathrm{H}_{2^{\prime \prime}}\right), 0.91(\mathrm{~d}, J=7.0 \mathrm{~Hz}$, $\left.3 \mathrm{H},\left(\mathrm{C}_{3}\right)_{2} \mathrm{CHCH}_{2}\right), 0.92\left(\mathrm{~d}, J=7.0 \mathrm{~Hz}, 3 \mathrm{H},\left(\mathrm{CH}_{3}\right)_{2} \mathrm{CHCH}_{2}\right)$, $1.20-1.25\left(\mathrm{~m}, 1 \mathrm{H},\left(\mathrm{CH}_{3}\right)_{2} \mathrm{CHCH}_{2}\right), 1.28-1.33\left(\mathrm{~m}, 2 \mathrm{H}, \mathrm{H}_{3}, 1 \times\right.$ $\left.\left(\mathrm{CH}_{3}\right)_{2} \mathrm{CHCH}_{2}\right), 1.39-1.56\left(\mathrm{~m}, 4 \mathrm{H}, \mathrm{H}_{5}, 1 \times \mathrm{H}_{1^{\prime \prime}}, 2 \times\right.$ $\left.\left(\mathrm{CH}_{3}\right)_{2} \mathrm{CHCH}_{2}\right), 1.62-1.70\left(\mathrm{~m}, 1 \mathrm{H}, \mathrm{H}_{1^{\prime \prime}}\right), 1.86-1.96(\mathrm{~m}, 2 \mathrm{H}, 2 \times$ $\left.\left(\mathrm{CH}_{3}\right)_{2} \mathrm{CHCH}_{2}\right), 2.50($ br s, $1 \mathrm{H}, \mathrm{OH}), 2.84($ br s, $1 \mathrm{H}, \mathrm{OH}), 3.11(\mathrm{td}$, $\left.J=10.4,2.8 \mathrm{~Hz}, 1 \mathrm{H}, \mathrm{H}_{6}\right), 3.18\left(\mathrm{td}, J=10.4,2.5 \mathrm{~Hz}, 1 \mathrm{H}, \mathrm{H}_{2}\right), 3.64$ (dd, $\left.J=10.6,8.1 \mathrm{~Hz}, 1 \mathrm{H}, 1 \times \mathrm{CH}_{2} \mathrm{OH}\right), 3.70(\mathrm{dd}, J=9.9,9.9 \mathrm{~Hz}, 1 \mathrm{H}$, $\left.\mathrm{H}_{4}\right), 3.96\left(\mathrm{dd}, J=10.6,3.4 \mathrm{~Hz}, 1 \mathrm{H}, 1 \times \mathrm{CH}_{2} \mathrm{OH}\right) ;{ }^{1} \mathrm{H} \operatorname{NMR}(600$ $\left.\mathrm{MHz}, \mathrm{C}_{6} \mathrm{D}_{6}\right) \delta 0.88-0.95\left(\mathrm{~m}, 15 \mathrm{H}, 3 \times \mathrm{H}_{2^{\prime \prime}}, 4 \times\left(\mathrm{CH}_{3}\right)_{2} \mathrm{CHCH}_{2}\right)$, 1.09-1.15 (m, $\left.1 \mathrm{H}, \mathrm{H}_{1^{\prime \prime \prime}}\right), 1.27-1.35\left(\mathrm{~m}, 2 \mathrm{H}, \mathrm{H}_{3}, \mathrm{H}_{1^{\prime \prime}}\right), 1.39-1.51$ $\left(\mathrm{m}, 4 \mathrm{H}, \mathrm{H}_{5}, 2 \times \mathrm{H}_{1^{\prime}}, 1 \times \mathrm{H}_{2^{\prime}}\right), 1.60\left(\right.$ br s, $\left.1 \mathrm{H}, \mathrm{CH}_{2} \mathrm{OH}\right), 1.69-1.76$ $\left(\mathrm{m}, 1 \mathrm{H}, \mathrm{H}_{1^{\prime \prime}}\right), 2.02-2.14\left(\mathrm{~m}, 2 \mathrm{H}, \mathrm{H}_{2^{\prime}}, \mathrm{H}_{2^{\prime \prime \prime}}\right), 2.66\left(\right.$ br s, $\left.1 \mathrm{H}, \mathrm{C}_{4} \mathrm{OH}\right)$, 2.95-3.01 (m, 1H, $\left.\mathrm{H}_{6}\right), 3.07-3.12\left(\mathrm{~m}, 1 \mathrm{H}, \mathrm{H}_{2}\right), 3.27-3.32(\mathrm{~m}, 1 \mathrm{H}, 1$ $\left.\times \mathrm{C}_{5} \mathrm{C}_{2} \mathrm{OH}\right), 3.51-3.56\left(\mathrm{~m}, 1 \mathrm{H}, \mathrm{H}_{4}\right), 3.60-3.65(\mathrm{~m}, 1 \mathrm{H}, 1 \times$ 
$\left.\mathrm{C}_{5} \mathrm{CH}_{2} \mathrm{OH}\right) ;{ }^{13} \mathrm{C}$ NMR $\left(125 \mathrm{MHz}, \mathrm{CDCl}_{3}\right) \delta 9.4\left(\mathrm{q}, \mathrm{C}_{2^{\prime \prime}}\right), 18.8(\mathrm{t}$, $\left.\mathrm{C}_{1^{\prime \prime}}\right), 21.1\left(\mathrm{q}, 2 \mathrm{C},\left(\mathrm{CH}_{3}\right)_{2} \mathrm{CHCH}_{2}\right), 24.1\left(\mathrm{~d},\left(\mathrm{CH}_{3}\right)_{2} \underline{\mathrm{C}} \mathrm{HCH}_{2}\right), 24.15$ $\left(\mathrm{q}, 2 \mathrm{C},\left(\mathrm{CH}_{3}\right)_{2} \mathrm{CHCH}_{2}\right), 24.19\left(\mathrm{~d},\left(\mathrm{CH}_{3}\right)_{2} \mathrm{CHCH}_{2}\right), 42.2(\mathrm{t}$, $\left.\left(\mathrm{CH}_{3}\right)_{2} \mathrm{CHC} \mathrm{H}_{2}\right), 42.7\left(\mathrm{t},\left(\mathrm{CH}_{3}\right)_{2} \mathrm{CHCH}_{2}\right), 49.1\left(\mathrm{~d}, \mathrm{C}_{3}\right), 50.6(\mathrm{~d}$, $\left.\mathrm{C}_{5}\right), 63.9\left(\mathrm{t}, \mathrm{CH}_{2} \mathrm{OH}\right), 74.0\left(\mathrm{~d}, \mathrm{C}_{6}\right), 74.5\left(\mathrm{~d}, \mathrm{C}_{4}\right), 75.8\left(\mathrm{~d}, \mathrm{C}_{2}\right)$; HRMS calcd for $\mathrm{C}_{16} \mathrm{H}_{32} \mathrm{O}_{3} \mathrm{Na}\left[(\mathrm{M}+\mathrm{Na})^{+}\right]$295.2249, found 295.2251 .

$\left(2 R^{*}, 3 R^{*}, 4 S^{*}, 5 S^{*}, 6 \mathrm{~S}^{*}\right)$-3-Ethyl-5-(hydroxymethyl)-2,6-diisobutyltetrahydro-2H-pyran-4-yl(2-hydroxyethyl)carbamate (20). Bicycle 5a (175 mg, $0.49 \mathrm{mmol})$ was dissolved in a 4:1 THF/ $\mathrm{H}_{2} \mathrm{O}$ mixture $(5 \mathrm{~mL}, 0.1 \mathrm{M})$; the solution was cooled to $0{ }^{\circ} \mathrm{C}$, and $\mathrm{NaBH}_{4}$ (75 mg, 1.96 mmol, 4 equiv) was added. Then, the reaction mixture was allowed to warm to rt and stirred for $16 \mathrm{~h}$. After that, the reaction was quenched with a saturated Rochelle salt aqueous solution $(5 \mathrm{~mL})$. The mixture was vigorously stirred for $16 \mathrm{~h}$ and then poured into a separatory funnel together with EtOAc $(10 \mathrm{~mL})$. The layers were separated; the aqueous layer was extracted with EtOAc $(3 \times 10$ $\mathrm{mL})$, and the combined organic layers were washed with brine $(50$ $\mathrm{mL}$ ), dried over $\mathrm{MgSO}_{4}$, filtered, concentrated, and purified by flash chromatography (12 $\mathrm{cm}$ of height of silica gel, $n$-hexane/EtOAc $30: 70)$ to yield compound $20(128 \mathrm{mg}, 70 \%)$ as an amorphous white solid: ${ }^{84} R_{f}=0.29$ (EtOAc/MeOH 80:20); ${ }^{1} \mathrm{H}$ NMR (500 MHz, $\left.\mathrm{CDCl}_{3}\right) \delta 0.85\left(\mathrm{t}, J=7.5 \mathrm{~Hz}, 3 \mathrm{H}, \mathrm{H}_{2^{\prime \prime}}\right), 0.87(\mathrm{~d}, J=6.6 \mathrm{~Hz}, 3 \mathrm{H}$, $\left.\left(\mathrm{CH}_{3}\right)_{2} \mathrm{CHCH}_{2}\right), 0.89\left(\mathrm{~d}, J=6.5 \mathrm{~Hz}, 3 \mathrm{H},\left(\mathrm{CH}_{3}\right)_{2} \mathrm{CHCH}_{2}\right), 0.92(\mathrm{~d}, J$ $\left.=6.8 \mathrm{~Hz}, 6 \mathrm{H},\left(\mathrm{CH}_{3}\right)_{2} \mathrm{CHCH}_{2}\right), 1.27-1.34\left(\mathrm{~m}, 2 \mathrm{H}, \mathrm{H}_{5}, \mathrm{H}_{1^{\prime \prime}}\right), 1.38-$ $1.52\left(\mathrm{~m}, 6 \mathrm{H}, \mathrm{H}_{3}, 2 \times \mathrm{H}_{1^{\prime}}, 2 \times \mathrm{H}_{1^{\prime \prime}}, \mathrm{H}_{1^{\prime \prime}}\right), 1.87-1.98(\mathrm{~m}, 2 \mathrm{H}$, $\left.\left(\mathrm{CH}_{3}\right)_{2} \mathrm{CHCH}_{2}\right), 3.26\left(\mathrm{td}, J=10.2,2.1 \mathrm{~Hz}, 1 \mathrm{H}, \mathrm{H}_{2}\right), 3.33-3.44(\mathrm{~m}$, $\left.2 \mathrm{H}, \mathrm{NC}_{2} \mathrm{CH}_{2} \mathrm{OH}\right), 3.51\left(\mathrm{td}, J=10.0,2.9 \mathrm{~Hz}, 1 \mathrm{H}, \mathrm{H}_{6}\right), 3.54(\mathrm{dd}, J=$ $\left.12.9,2.5 \mathrm{~Hz}, 1 \mathrm{H}, \mathrm{C}_{5} \mathrm{CH}_{2} \mathrm{OH}\right), 3.61-3.65\left(\mathrm{~m}, 1 \mathrm{H}, \mathrm{C}_{5} \mathrm{CH}_{2} \mathrm{OH}\right), 3.74$ $\left(\mathrm{t}, J=5.0 \mathrm{~Hz}, 2 \mathrm{H}, \mathrm{NCH}_{2} \underline{\mathrm{CH}}_{2} \mathrm{OH}\right), 4.92(\mathrm{dd}, J=10.5,10.5 \mathrm{~Hz}, 1 \mathrm{H}$ $\left.\mathrm{H}_{4}\right), 5.25(\mathrm{t}, J=5.7 \mathrm{~Hz}, 1 \mathrm{H}, \mathrm{NH}) ;{ }^{13} \mathrm{C}$ NMR $\left(125 \mathrm{MHz}, \mathrm{CDCl}_{3}\right) \delta$ $9.4\left(\mathrm{q}, \mathrm{C}_{2^{\prime \prime}}\right), 19.7\left(\mathrm{t}, \mathrm{C}_{1^{\prime \prime}}\right), 21.05\left(\mathrm{q},\left(\mathrm{CH}_{3}\right)_{2} \mathrm{CHCH}_{2}\right), 21.08(\mathrm{q}$, $\left.\left(\underline{\mathrm{CH}}_{3}\right)_{2} \mathrm{CHCH}_{2}\right), 24.1 \quad\left(\mathrm{q}, \quad\left(\underline{\mathrm{CH}}_{3}\right)_{2} \mathrm{CHCH}_{2}\right), 24.16$ (d, $\left.\left(\mathrm{CH}_{3}\right)_{2} \mathrm{CHCH}_{2}\right), 24.17\left(\mathrm{q},\left(\mathrm{CH}_{3}\right)_{2} \mathrm{CHCH}_{2}\right), 24.3$ (d, $\left.\left.\left(\mathrm{CH}_{3}\right)_{2} \underline{\mathrm{C}} \mathrm{HCH}_{2}\right), 41.9\left(\mathrm{t},\left(\mathrm{CH}_{3}\right)_{2} \mathrm{CH}_{\mathrm{CH}}\right)_{2}\right), 42.2\left(\mathrm{t},\left(\mathrm{CH}_{3}\right)_{2} \mathrm{CH}_{2} \mathrm{H}_{2}\right)$, $43.6\left(\mathrm{t}, \quad \mathrm{NCH}_{2} \mathrm{CH}_{2} \mathrm{OH}\right), 46.8\left(\mathrm{~d}, \mathrm{C}_{3}\right), 50.6\left(\mathrm{~d}, \mathrm{C}_{5}\right), 58.1(\mathrm{t}$, $\left.\mathrm{C}_{5} \mathrm{CH}_{2} \mathrm{OH}\right), 62.3\left(\mathrm{t}, \mathrm{NCH}_{2} \mathrm{CH}_{2} \mathrm{OH}\right), 73.1\left(\mathrm{~d}, \mathrm{C}_{4}\right), 73.8\left(\mathrm{~d}, \mathrm{C}_{6}\right), 75.7$ $\left(\mathrm{d}, \mathrm{C}_{2}\right), 158.8(\mathrm{~s}, \mathrm{OC}(\mathrm{O}) \mathrm{N}) ; \mathrm{MS}(\mathrm{EI}) \mathrm{m} / z$ (relative intensity) 302 $(\mathrm{M}-i-\mathrm{Bu})^{+}(3), 254\left(\mathrm{M}-1-\mathrm{OC}(\mathrm{O}) \mathrm{NHCH}_{2} \mathrm{CH}_{2} \mathrm{OH}\right)^{+}(5), 223$ $\left(\mathrm{M}-1 \text { - carbamate }-\mathrm{CH}_{2} \mathrm{OH}\right)^{+}(31), 197(\mathrm{M}-1-i-\mathrm{Bu}-$

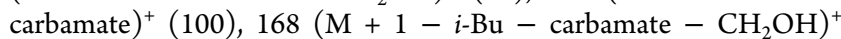
(41), $141(\mathrm{M}-2 \mathrm{i}-\mathrm{Bu}-\text { carbamate })^{+}(9), 111(\mathrm{M}+1-2 i-\mathrm{Bu}-$ carbamate $\left.-\mathrm{CH}_{2} \mathrm{OH}\right)^{+}(12)$; HRMS calcd for $\mathrm{C}_{15} \mathrm{H}_{28} \mathrm{NO}_{5}[(\mathrm{M}-i$ $\mathrm{Bu}^{+}$] 302.1967, found 302.1961.

Synthesis of 3-(N-Acyl oxazolidin-2-one)-THPs 21. (R)-4Benzyl-3-[(2S,3R,4S,5S,6R)-5-ethyl-4-hydroxy-2,6-dimethyltetrahydro-2H-pyran-3-carbonyl]oxazolidin-2-one (21a). Aldol $2 \mathrm{i}(26 \mathrm{mg}$, $83 \mu \mathrm{mol}$ ) and acetaldehyde ( $37 \mu \mathrm{L}$ of a $3.3 \mathrm{M}$ solution in DCM, 125 $\mu$ mol, 1.5 equiv) were subjected to the general procedure for the synthesis of bicycles 5 (two-step EAP) and yielded, after purification by flash chromatography (18 cm of height of silica gel, $n$-hexane/ EtOAc 70:30), title compound 21a (3 mg, 9\%, >95:5 dr) and previously described $5 \mathrm{ag}(19 \mathrm{mg}, 62 \%,>95: 5 \mathrm{dr}) .21 \mathrm{a}$ : thick colorless oil; $R_{f}=0.43\left(n\right.$-hexane/EtOAc 60:40); $[\alpha]_{\mathrm{D}}^{25}-66.0\left(c 0.6, \mathrm{CHCl}_{3}\right)$; ${ }^{1} \mathrm{H}$ NMR $\left(500 \mathrm{MHz}, \mathrm{CDCl}_{3}\right) \delta 0.93\left(\mathrm{t}, J=7.6 \mathrm{~Hz}, 3 \mathrm{H}, \mathrm{C}_{5^{\prime}} \mathrm{CH}_{2} \mathrm{CH}_{3}\right)$, $1.18\left(\mathrm{~d}, J=5.9 \mathrm{~Hz}, 3 \mathrm{H}, \mathrm{C}_{2^{\prime}} \mathrm{CH}_{3}\right), 1.26\left(\mathrm{~d}, J=6.2 \mathrm{~Hz}, 3 \mathrm{H}, \mathrm{C}_{6} \mathrm{CH}_{3}\right)$, $1.29-1.35\left(\mathrm{~m}, 1 \mathrm{H}, \mathrm{H}_{5^{\prime}}\right), 1.54-1.61\left(\mathrm{~m}, 1 \mathrm{H}, \mathrm{C}_{5^{\prime}} \underline{\mathrm{CH}}_{2} \mathrm{CH}_{3}\right), 1.70-1.77$ $\left(\mathrm{m}, 1 \mathrm{H}, \mathrm{C}_{5}, \mathrm{CH}_{2} \mathrm{CH}_{3}\right), 2.16(\mathrm{~d}, J=9.9 \mathrm{~Hz}, 1 \mathrm{H}, \mathrm{OH}), 2.82(\mathrm{dd}, J=$ 13.7, $9.5 \mathrm{~Hz}, 1 \mathrm{H}, \mathrm{C}_{4} \mathrm{CH}_{2}$ ), 3.34 (dd, $J=13.7,3.5 \mathrm{~Hz}, 1 \mathrm{H}, \mathrm{C}_{4} \mathrm{CH}_{2}$ ), $3.43\left(\mathrm{dq}, J=10.0,6.2 \mathrm{~Hz}, 1 \mathrm{H}, \mathrm{H}_{6^{\prime}}\right), 3.76(\mathrm{dq}, J=8.9,6.0 \mathrm{~Hz}, 1 \mathrm{H}$, $\left.\mathrm{H}_{2^{\prime}}\right), 3.80-3.83\left(\mathrm{~m}, 1 \mathrm{H}, \mathrm{H}_{3^{\prime}}\right), 3.84(\mathrm{ddd} J=9.8,9.8,9.8 \mathrm{~Hz}, 1 \mathrm{H}$, $\left.\mathrm{H}_{4^{\prime}}\right), 4.16-4.23\left(\mathrm{~m}, 2 \mathrm{H}, \mathrm{H}_{5}\right), 4.68-4.72\left(\mathrm{~m}, 1 \mathrm{H}, \mathrm{H}_{4}\right), 7.25-7.27(\mathrm{~m}$, $\left.2 \mathrm{H}, \mathrm{H}_{3^{\prime \prime}}, \mathrm{H}_{5^{\prime \prime}}\right), 7.28-7.29\left(\mathrm{~m}, 1 \mathrm{H}, \mathrm{H}_{4^{\prime \prime}}\right), 7.32-7.35\left(\mathrm{~m}, 2 \mathrm{H}, \mathrm{H}_{2^{\prime \prime}}, \mathrm{H}_{6^{\prime \prime}}\right)$; ${ }^{13} \mathrm{C}$ NMR $\left(125 \mathrm{MHz}, \mathrm{CDCl}_{3}\right) \delta 9.8\left(\mathrm{q}, \mathrm{C}_{5^{\prime}} \mathrm{CH}_{2} \mathrm{CH}_{3}\right), 19.2(\mathrm{t}$, $\left.\mathrm{C}_{5^{\prime}} \underline{\mathrm{CH}_{2}} \mathrm{CH}_{3}\right), 19.5\left(\mathrm{q}, \mathrm{C}_{6^{\prime}} \underline{\mathrm{CH}_{3}}\right), 19.8\left(\mathrm{q}, \mathrm{C}_{2^{\prime}} \underline{\mathrm{CH}_{3}}\right), 37.8\left(\mathrm{t}, \mathrm{C}_{4} \underline{\mathrm{CH}_{2}}\right)$, 50.9 (d, $\left.\mathrm{C}_{5^{\prime}}\right), 55.3\left(\mathrm{~d}, \mathrm{C}_{3^{\prime}}\right), 56.1\left(\mathrm{~d}, \mathrm{C}_{4}\right), 66.3\left(\mathrm{t}, \mathrm{C}_{5}\right), 73.6\left(\mathrm{~d}, \mathrm{C}_{2^{\prime}}\right)$, $74.4\left(\mathrm{~d}, \mathrm{C}_{4^{\prime}}\right), 75.0\left(\mathrm{~d}, \mathrm{C}_{6^{\prime}}\right), 127.5\left(\mathrm{~d}, \mathrm{C}_{4^{\prime \prime}}\right), 129.1\left(\mathrm{~d}, 2 \mathrm{C}, \mathrm{C}_{2^{\prime \prime}}, \mathrm{C}_{6^{\prime \prime}}\right)$, $129.7\left(\mathrm{~d}, 2 \mathrm{C}, \mathrm{C}_{3^{\prime \prime}}, \mathrm{C}_{5^{\prime \prime}}\right), 135.3\left(\mathrm{~s}, \mathrm{C}_{1^{\prime \prime}}\right), 154.5\left(\mathrm{~s}, \mathrm{C}_{2}\right), 174.2(\mathrm{~s}$, $\left.\mathrm{C}_{3^{\prime}} \mathrm{C}(\mathrm{O}) \mathrm{N}\right) ; \mathrm{MS}(\mathrm{EI}) \mathrm{m} / z$ (relative intensity) $361(\mathrm{M})^{+}(4), 344(\mathrm{M}$ $-\mathrm{OH})^{+}(5), 343\left(\mathrm{M}-\mathrm{H}_{2} \mathrm{O}\right)^{+}(24), 228(9), 185(\mathrm{M}$ - oxazolidin-2- one $)^{+}(13), 184(12), 157$ ( $\mathrm{M}-\mathrm{N}$-acyl oxazolidin-2-one $)^{+}$(3), 91 (100); HRMS calcd for $\mathrm{C}_{20} \mathrm{H}_{27} \mathrm{NO}_{5}\left[(\mathrm{M})^{+}\right]$361.1889, found 361.1903.

(S)-3-[(2R,3S, 4R,5R,6S)-5-Ethyl-4-hydroxy-2,6-dimethyltetrahydro-2H-pyran-3-carbonyl)-4-isopropyloxazolidin-2-one (21b). Aldol $2 \mathrm{k}(58 \mathrm{mg}, 0.22 \mathrm{mmol})$ and acetaldehyde $(0.1 \mathrm{~mL}$ of a 3.3 $\mathrm{M}$ solution in DCM, $0.33 \mathrm{mmol}, 1.5$ equiv) were subjected to the general procedure for the synthesis of bicycles 5 (two-step EAP) and yielded, after purification by flash chromatography $(28 \mathrm{~cm}$ of height of silica gel, $n$-hexane/EtOAc 85:15), title compound $21 \mathrm{~b}(11 \mathrm{mg}, 16 \%$, $>95: 5 \mathrm{dr})$ and previously described bicycle $5 \mathrm{ah}(29 \mathrm{mg}, 43 \%, 92: 8$ dr). 21b: thick colorless oil; $R_{f}=0.37$ ( $n$-hexane/EtOAc 60:40); $[\alpha]_{\mathrm{D}}^{25}+97.9\left(c 0.9, \mathrm{CHCl}_{3}\right) ;{ }^{1} \mathrm{H}$ NMR $\left(500 \mathrm{MHz}, \mathrm{CDCl}_{3}\right) \delta 0.91(\mathrm{t}$, $\left.J=7.6 \mathrm{~Hz}, 3 \mathrm{H}, \mathrm{CH}_{3} \mathrm{CH}_{2} \mathrm{C}_{5^{\prime}}\right), 0.93(\mathrm{t}, J=7.3 \mathrm{~Hz}, 6 \mathrm{H}, 2 \times$ $\left.\left(\mathrm{CH}_{3}\right)_{2} \mathrm{CHC}_{3}\right), 1.16\left(\mathrm{~d}, J=5.8 \mathrm{~Hz}, 3 \mathrm{H}, \mathrm{CH}_{3} \mathrm{C}_{2^{\prime}}\right), 1.25(\mathrm{~d}, J=6.2 \mathrm{~Hz}$, $\left.3 \mathrm{H}, \mathrm{CH}_{3} \mathrm{C}_{6^{\prime}}\right), 1.26-1.30\left(\mathrm{~m}, 1 \mathrm{H}, \mathrm{H}_{5^{\prime}}\right), 1.51-1.57(\mathrm{~m}, 1 \mathrm{H}, 1 \times$ $\left.\mathrm{CH}_{3} \mathrm{CH}_{2} \mathrm{C}_{5^{\prime}}\right), 1.66-1.74\left(\mathrm{~m}, 1 \mathrm{H}, 1 \times \mathrm{CH}_{3} \mathrm{CH}_{2} \mathrm{C}_{5^{\prime}}\right), 2.16(\mathrm{~d}, J=10.4$ $\mathrm{Hz}, 1 \mathrm{H}, \mathrm{OH}){ }^{85} 2.43-2.49\left(\mathrm{~m}, 1 \mathrm{H},\left(\mathrm{CH}_{3}\right)_{2} \mathrm{CHC}_{3}\right), 3.39(\mathrm{dq}, J=$ $\left.10.0,6.0 \mathrm{~Hz}, 1 \mathrm{H}, \mathrm{H}_{6^{\prime}}\right), 3.72\left(\mathrm{dq}, J=9.2,6.1 \mathrm{~Hz}, 1 \mathrm{H}, \mathrm{H}_{2^{\prime}}\right), 3.75(\mathrm{dd}, J$ $\left.=10.0,9.4 \mathrm{~Hz}, 1 \mathrm{H}, \mathrm{H}_{4^{\prime}}\right), 3.82\left(\mathrm{dd}, J=9.3,9.3 \mathrm{~Hz}, 1 \mathrm{H}, \mathrm{H}_{3^{\prime}}\right), 4.24(\mathrm{dd}$, $\left.J=9.2,2.8 \mathrm{~Hz}, 1 \mathrm{H}, \mathrm{H}_{5}\right), 4.29\left(\mathrm{dd}, J=9.2,7.7 \mathrm{~Hz}, 1 \mathrm{H}, \mathrm{H}_{5}\right), 4.46(\mathrm{ddd}$, $\left.J=7.9,3.7,2.6 \mathrm{~Hz}, 1 \mathrm{H}, \mathrm{H}_{4}\right) ;{ }^{13} \mathrm{C} \mathrm{NMR}\left(125 \mathrm{MHz} \mathrm{CDCl}_{3}\right) \delta 9.9(\mathrm{q}$, $\left.\mathrm{CH}_{3} \mathrm{CH}_{2} \mathrm{C}_{5^{\prime}}\right), 14.8\left(\mathrm{q}, 1 \times\left(\mathrm{CH}_{3}\right)_{2} \mathrm{CHC}_{3}\right), 18.1(\mathrm{q}, 1 \times$ $\left.\left(\underline{\mathrm{CH}}_{3}\right)_{2} \mathrm{CHC}_{3}\right), 19.3\left(\mathrm{t}, \mathrm{CH}_{3} \underline{\mathrm{CH}}_{2} \mathrm{C}_{5^{\prime}}\right), 19.5\left(\mathrm{q}, \mathrm{CH}_{3} \mathrm{C}_{6^{\prime}}\right), 19.8$ (q, $\left.\mathrm{CH}_{3} \mathrm{C}_{2^{\prime}}\right), 28.7\left(\mathrm{~d},\left(\mathrm{CH}_{3}\right)_{2} \mathrm{CHC}_{3}\right), 51.1\left(\mathrm{~d}, \mathrm{C}_{5^{\prime}}\right), 55.1\left(\mathrm{~d}, \mathrm{C}_{3^{\prime}}\right), 59.4(\mathrm{~d}$, $\left.\mathrm{C}_{4}\right), 63.7\left(\mathrm{t}, \mathrm{C}_{5}\right), 73.5\left(\mathrm{~d}, \mathrm{C}_{2^{\prime}}\right), 74.5\left(\mathrm{~d}, \mathrm{C}_{4^{\prime}}\right), 75.0\left(\mathrm{~d}, \mathrm{C}_{6^{\prime}}\right), 155.2(\mathrm{~s}$, $\left.\mathrm{C}_{2}\right), 174.0\left(\mathrm{~s}, \mathrm{C}_{3^{\prime}} \mathrm{C}(\mathrm{O}) \mathrm{N}\right) ; \mathrm{MS}(\mathrm{EI}) \mathrm{m} / z$ (relative intensity) $297(\mathrm{M}$ $-\mathrm{H}-\mathrm{Me})^{+}(1), 295\left(\mathrm{M}-\mathrm{H}_{2} \mathrm{O}\right)^{+}(26), 283(\mathrm{M}-\mathrm{Et}-\mathrm{H})^{+}(1),{ }^{86}$ $271(\mathrm{M}+1-i-\mathrm{Pr})^{+}(1), 228(\mathrm{M}+\mathrm{H}-\mathrm{Et}-\mathrm{Me}-i-\mathrm{Pr})^{+}(2),{ }^{87} 185$ $(\mathrm{M} \text { - oxazolidin-2-one })^{+}(4), 157(\mathrm{M}-\mathrm{N} \text {-acyl oxazolidin-2-one })^{+}$ $(1){ }^{88} 156$ ( $N$-acyl oxazolidin-2-one $)^{+}(5) ;^{88}$ HRMS calcd for $\mathrm{C}_{16} \mathrm{H}_{25} \mathrm{NO}_{4}\left[\left(\mathrm{M}-\mathrm{H}_{2} \mathrm{O}\right)^{+}\right] 295.1784$, found 295.1782 .

(S)-3-[(2R,3S,4R,5R,6S)-5-Ethyl-4-hydroxy-6-methyl-2-phenethyltetrahydro-2H-pyran-3-carbonyl]-4-isopropyloxazolidin-2-one (21c). Aldol 21 (43 mg, $0.12 \mathrm{mmol})$ and acetaldehyde $(0.05 \mathrm{~mL}$ of a $3.3 \mathrm{M}$ solution in DCM, $0.18 \mathrm{mmol}, 1.5$ equiv) were subjected to the general procedure for the synthesis of bicycles 5 (two-step EAP) and yielded, after purification by flash chromatography $(28 \mathrm{~cm}$ of height of silica gel, $n$-hexane/EtOAc 90:10), title compound $21 \mathrm{c}(6 \mathrm{mg}, 12 \%$, $>95: 5 \mathrm{dr})$ and previously described bicycle 5 ai $(22 \mathrm{mg}, 45 \%,>95: 5$ dr). 21c: white solid (probably crystalline); $R_{f}=0.63$ ( $n$-hexane/ EtOAc 70:30, three times); $[\alpha]_{\mathrm{D}}^{25}+61.9\left(\mathrm{c} 0.3, \mathrm{CHCl}_{3}\right) ;{ }^{1} \mathrm{H}$ NMR $\left(500 \mathrm{MHz}, \mathrm{CDCl}_{3}\right) \delta 0.89-0.92\left(\mathrm{~m}, 9 \mathrm{H}, 2 \times\left(\mathrm{CH}_{3}\right)_{2} \mathrm{CH}, \mathrm{CH}_{3} \mathrm{CH}_{2}\right)$, $1.27-1.32\left(\mathrm{~m}, 1 \mathrm{H}, \mathrm{H}_{5^{\prime}}\right), 1.29\left(\mathrm{~d}, J=6.0 \mathrm{~Hz}, 3 \mathrm{H}, \mathrm{CH}_{3} \mathrm{C}_{6^{\prime}}\right), 1.56-1.61$ (m, $1 \mathrm{H}, \mathrm{CH}_{3} \mathrm{C}_{2}$ ), $1.62-1.78$ (m, 3H, $\mathrm{C}_{2} \mathrm{CH}_{2} \mathrm{Ph}, \mathrm{CH}_{3} \mathrm{C}_{2}$ ), 2.15 (d, $J=10.7 \mathrm{~Hz}, 1 \mathrm{H}, \mathrm{OH}), 2.40-2.48\left(\mathrm{~m}, 1 \mathrm{H},\left(\mathrm{CH}_{3}\right)_{2} \mathrm{CH}\right), 2.57-2.63$ (m, $\left.1 \mathrm{H}, \mathrm{CH}_{2} \underline{\mathrm{C}}_{2} \mathrm{Ph}\right), 2.85-2.91\left(\mathrm{~m}, 1 \mathrm{H}, \mathrm{CH}_{2} \underline{\mathrm{C}}_{2} \mathrm{Ph}\right), 3.36$ (dq, $J=$ $\left.10.0,6.0 \mathrm{~Hz}, 1 \mathrm{H}, \mathrm{H}_{6^{\prime}}\right), 3.61\left(\mathrm{td}, J=9.5,2.4 \mathrm{~Hz}, 1 \mathrm{H}, \mathrm{H}_{2^{\prime}}\right), 3.73(\mathrm{ddd}, J$ $\left.=10.4,10.4,10.4 \mathrm{~Hz}, 1 \mathrm{H}, \mathrm{H}_{4^{\prime}}\right), 3.89\left(\mathrm{dd}, J=9.9,9.9 \mathrm{~Hz}, 1 \mathrm{H}, \mathrm{H}_{3^{\prime}}\right)$, 4.19-4.26 (m, 2H, $\left.\mathrm{H}_{5}\right), 4.39-4.42\left(\mathrm{~m}, 1 \mathrm{H}, \mathrm{H}_{4}\right), 7.16-7.19(\mathrm{~m}, 3 \mathrm{H}$, $\mathrm{Ph}), 7.24-7.28(\mathrm{~m}, 2 \mathrm{H}, \mathrm{Ph}) ;{ }^{13} \mathrm{C}$ NMR $\left(125 \mathrm{MHz}, \mathrm{CDCl}_{3}\right) \delta 9.9(\mathrm{q}$, $\left.\underline{\mathrm{CH}}_{3} \mathrm{CH}_{2}\right), 14.8\left(\mathrm{q},\left(\underline{\mathrm{CH}}_{3}\right)_{2} \mathrm{CH}\right), 18.1\left(\mathrm{q},\left(\underline{\mathrm{CH}}_{3}\right)_{2} \mathrm{CH}\right), 19.3(\mathrm{t}$,

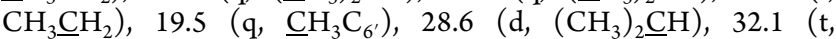
$\left.\mathrm{CH}_{2} \mathrm{CH}_{2} \mathrm{Ph}\right), 35.8\left(\mathrm{t}, \underline{\mathrm{CH}_{2}} \mathrm{CH}_{2} \mathrm{Ph}\right), 51.5\left(\mathrm{~d}, \mathrm{C}_{5^{\prime}}\right), 53.8\left(\mathrm{~d}, \mathrm{C}_{3^{\prime}}\right)$, $59.4\left(\mathrm{~d}, \mathrm{C}_{4}\right), 63.6\left(\mathrm{t}, \mathrm{C}_{5}\right), 74.9\left(\mathrm{~d}, \mathrm{C}_{4^{\prime}}\right), 75.1\left(\mathrm{~d}, \mathrm{C}_{6^{\prime}}\right), 76.7\left(\mathrm{~d}, \mathrm{C}_{2^{\prime}}\right)$, 126.0 (d, Ph), 128.5 (d, 2C, Ph), 128.6 (d, 2C, Ph), 142.3 (s, Ph), $155.2\left(\mathrm{~s}, \mathrm{C}_{2}\right), 174.0\left(\mathrm{~s}, \mathrm{C}_{3^{\prime}} \mathrm{C}(\mathrm{O}) \mathrm{N}\right) ; \mathrm{MS}(\mathrm{EI}) \mathrm{m} / z$ (relative intensity) $403(\mathrm{M})^{+}(4), 388(\mathrm{M}-\mathrm{Me})^{+}(6), 385\left(\mathrm{M}-\mathrm{H}_{2} \mathrm{O}\right)^{+}(12), 316(\mathrm{M}-$ $\mathrm{H}-\mathrm{Et}-\mathrm{Me}-i-\mathrm{Pr})^{+}(3), 298\left(\mathrm{M}-\mathrm{CH}_{2} \mathrm{CH}_{2} \mathrm{Ph}\right)^{+}(4), 275(\mathrm{M}-$ oxazolidin-2-one $)^{+}(2), 256\left(\mathrm{M}-\mathrm{H}-\mathrm{H}_{2} \mathrm{O}-\right.$ oxazolidin-2-one) (59), $248(\mathrm{M}+\mathrm{H}-\mathrm{N} \text {-acyl oxazolidin-2-one })^{+}(6),{ }^{89} 158(\mathrm{~N}$-acyl oxazolidin-2-one +2$)^{+}(9),{ }^{89} 130$ (oxazolidin-2-one +2$)^{+}(74) ;^{89}$ HRMS calcd for $\mathrm{C}_{23} \mathrm{H}_{33} \mathrm{NO}_{5}\left[(\mathrm{M})^{+}\right]$403.2359, found 403.2353.

$(4 R, 5 S)-3-[(2 S, 3 R, 4 S, 5 S, 6 R)-2,6-D i b u t y l-5$-ethyl-4-hydroxytetrahydro-2H-pyran-3-carbonyl]-4-methyl-5-phenyloxazolidin-2-one (21d). Aldol $2 \mathrm{~m}(22 \mathrm{mg}, 60 \mathrm{mmol})$ was subjected to the general procedure for the synthesis of bicycles $\mathbf{5}$ (two-step EAP) and yielded, after purification by flash chromatography $(18 \mathrm{~cm}$ of height of silica gel, $600 \mathrm{~mL}$ of $n$-hexane/EtOAc 90:10 to remove nonpolar impurities ${ }^{90}$ and then $200 \mathrm{~mL}$ of EtOAc), title compound 21d (2.7 
$\mathrm{mg}, 10 \%,>95: 5 \mathrm{dr}$ ) as a colorless oil: $R_{f}=0.69$ (EtOAc); $[\alpha]^{25}{ }_{\mathrm{D}}$ $-13.9\left(c 0.3, \mathrm{CHCl}_{3}\right) ;{ }^{1} \mathrm{H}$ NMR $\left(500 \mathrm{MHz}, \mathrm{CDCl}_{3}\right) \delta 0.81(\mathrm{t}, J=7.2$ $\left.\mathrm{Hz}, 3 \mathrm{H}, \mathrm{CH}_{3} \mathrm{CH}_{2} \mathrm{CH}_{2} \mathrm{CH}_{2}\right), 0.85-0.94\left(\mathrm{~m}, 6 \mathrm{H}, \mathrm{CH}_{3} \mathrm{CH}_{2} \mathrm{C}_{5^{\prime}}\right.$ and $\left.\mathrm{CH}_{3} \mathrm{CH}_{2} \mathrm{CH}_{2} \mathrm{CH}_{2}\right), 1.14-1.55\left(\mathrm{~m}, 13 \mathrm{H}, \mathrm{H}_{5^{\prime}}, 12 \mathrm{H}\right.$ from $\left.\mathrm{CH}_{2}\right), 1.63-$ $1.68\left(\mathrm{~m}, 1 \mathrm{H}, 1 \mathrm{H}\right.$ from $\left.\mathrm{CH}_{2}\right), 1.69-1.75\left(\mathrm{~m}, 1 \mathrm{H}, 1 \times \mathrm{CH}_{3} \mathrm{CH}_{2} \mathrm{C}_{5^{\prime}}\right)$, $2.01(\mathrm{~d}, J=9.2 \mathrm{~Hz}, 1 \mathrm{H}, \mathrm{OH}), 3.20\left(\mathrm{td}, J=9.7,2.1 \mathrm{~Hz}, 1 \mathrm{H}, \mathrm{H}_{6^{\prime}}\right), 3.46$ $\left(\mathrm{td}, J=9.4,2.6 \mathrm{~Hz}, 1 \mathrm{H}, \mathrm{H}_{2^{\prime}}\right), 3.77-3.85\left(\mathrm{~m}, 1 \mathrm{H}, \mathrm{H}_{4^{\prime}}\right), 3.85-3.88(\mathrm{~m}$, $\left.1 \mathrm{H}, \mathrm{H}_{3^{\prime}}\right), 4.47\left(\mathrm{qd}, J=6.3,2.8 \mathrm{~Hz}, 1 \mathrm{H}, \mathrm{H}_{4}\right), 5.11(\mathrm{~d}, J=2.5 \mathrm{~Hz}, 1 \mathrm{H}$, $\left.\mathrm{H}_{5}\right), 7.27-7.28(\mathrm{~m}, 1 \mathrm{H}, \mathrm{Ph}), 7.30-7.36(\mathrm{~m}, 1 \mathrm{H}, \mathrm{Ph}), 7.38-7.43(\mathrm{~m}$, $3 \mathrm{H}) ;{ }^{13} \mathrm{C}$ NMR $\left(150 \mathrm{MHz}, \mathrm{CDCl}_{3}\right) \delta 9.8\left(\mathrm{q}, \mathrm{CH}_{3} \mathrm{CH}_{2} \mathrm{C}_{5^{\prime}}\right), 14.1$ (q, $\left.\mathrm{CH}_{3} \mathrm{CH}_{2} \mathrm{CH}_{2} \mathrm{CH}_{2} \mathrm{C}_{2^{\prime}}\right), 14.3$ (q, $\left.\mathrm{CH}_{3} \mathrm{CH}_{2} \mathrm{CH}_{2} \mathrm{CH}_{2} \mathrm{C}_{2^{\prime}}\right), 19.0$ (t,

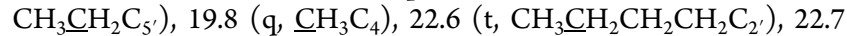
(t, $\left.\mathrm{CH}_{3} \mathrm{CH}_{2} \mathrm{CH}_{2} \mathrm{CH}_{2} \mathrm{C}_{2^{\prime}}\right), 27.75\left(\mathrm{t}, \mathrm{CH}_{3} \mathrm{CH}_{2} \mathrm{CH}_{2} \mathrm{CH}_{2} \mathrm{C}_{2^{\prime}}\right), 27.82(\mathrm{t}$, $\left.\mathrm{CH}_{3} \mathrm{CH}_{2} \mathrm{CH}_{2} \mathrm{CH}_{2} \mathrm{C}_{2^{\prime}}\right), 32.5\left(\mathrm{t}, \mathrm{CH}_{3} \mathrm{CH}_{2} \mathrm{CH}_{2} \mathrm{CH}_{2} \mathrm{C}_{2^{\prime}}\right), 33.6(\mathrm{t}$, $\left.\mathrm{CH}_{3} \mathrm{CH}_{2} \mathrm{CH}_{2} \mathrm{CH}_{2} \mathrm{C}_{2^{\prime}}\right), 49.2\left(\mathrm{~d}, \mathrm{C}_{5^{\prime}}\right), 54.4\left(\mathrm{~d}, \mathrm{C}_{3^{\prime}}\right), 58.7\left(\mathrm{~d}, \mathrm{C}_{4}\right)$, $74.7\left(\mathrm{~d}, \mathrm{C}_{4^{\prime}}\right), 77.4\left(\mathrm{~d}, \mathrm{C}_{2^{\prime}}\right), 78.3\left(\mathrm{~d}, \mathrm{C}_{6^{\prime}}\right), 81.8\left(\mathrm{~d}, \mathrm{C}_{5}\right), 125.1(\mathrm{~d}, 2 \mathrm{C}$, $\mathrm{Ph}), 129.3$ (d, 2C, Ph), $129.5(\mathrm{~d}, \mathrm{Ph}), 137.6$ (s, Ph), $154.1\left(\mathrm{~s}, \mathrm{C}_{2}\right)$, $174.3\left(\mathrm{~s}, \mathrm{C}_{3}, \mathrm{C}(\mathrm{O}) \mathrm{N}\right) ; \mathrm{MS}(\mathrm{EI}) \mathrm{m} / z$ (relative intensity) $445(\mathrm{M})^{+}(1)$, $428(\mathrm{M}-\mathrm{OH})^{+}(18), 427\left(\mathrm{M}-\mathrm{H}_{2} \mathrm{O}\right)^{+}(61), 388(\mathrm{M}-\mathrm{Bu})^{+}(22)$, $373(\mathrm{M}-\mathrm{Bu}-\mathrm{Me})^{+}(2), 359(\mathrm{M}-\mathrm{Bu}-\mathrm{Et})^{+}(1), 339(\mathrm{M}-\mathrm{Et}-$ $\mathrm{Ph})^{+}(21), 302(\mathrm{M}-2 \mathrm{Bu}-\mathrm{Et})^{+}(1), 250\left(\mathrm{M}-\mathrm{Et}-\mathrm{Bu}-\mathrm{H}_{2} \mathrm{O}-\right.$ $\mathrm{Me}-\mathrm{Ph})^{+}(100), 240(\mathrm{M}+1-N \text {-acyl oxazolidin-2-one })^{+}(3), 204$ $(\mathrm{N} \text {-acyl oxazolidin-2-one })^{+}(4), 178$ (oxazolidin-2-one +2$)^{+}(49){ }^{91}$ HRMS calcd for $\mathrm{C}_{26} \mathrm{H}_{37} \mathrm{NO}_{4}\left[\left(\mathrm{M}-\mathrm{H}_{2} \mathrm{O}\right)^{+}\right]$427.2723, found 427.2704.

\section{ASSOCIATED CONTENT}

\section{(5) Supporting Information}

The Supporting Information is available free of charge on the ACS Publications website at DOI: 10.1021/acs.joc.8b01182.

Molecule index that correlates the numbering of the molecules discussed herein with those revealed in our previous report; ${ }^{30}$ list of the products subjected to biological evaluation; NMR analysis of the minor diastereoisomer obtained during the synthesis of bicycle $\mathbf{5 a}$; comparison of representative signals of $\mathbf{1 b}-\mathbf{B r}, \mathbf{5 b}$, and $7 \mathbf{b}-\mathbf{B r}$ in NMR spectra; NMR analysis of bicycle $\mathbf{5 e}$ and its minor diastereoisomers, as well as a mechanistic proposal for their production; mechanistic proposal and NMR evolution of the conversion of anti-aldol 9a into products $10 \mathrm{~b}$ and 12; helpful information for the identification of bicycles 5 and THPs-Xc 21; chiral HPLC chromatograms; screening of Lewis acids for the enantiomeric version of Prins cyclization; DFT calculation results; copies of ${ }^{1} \mathrm{H}$ and ${ }^{13} \mathrm{C}$ NMR spectra for new products; and two-dimensional NMR spectra for representative products (PDF)

\section{AUTHOR INFORMATION}

\section{Corresponding Authors}

*E-mail: salvmen@ull.edu.es.

*E-mail: vmartin@ull.edu.es.

ORCID $\odot$

Sergio J. Álvarez-Méndez: 0000-0002-2473-9622

Víctor S. Martín: 0000-0003-0300-9636

\section{Present Address}

"S.J.Á.-M.: Sección de Ingeniería Agraria, Escuela Politécnica Superior de Ingeniería, Universidad de La Laguna (ULL), Carretera San Miguel de Geneto 2, 38296 San Cristóbal de La Laguna, Tenerife, Spain.

Notes

The authors declare no competing financial interest.

\section{ACKNOWLEDGMENTS}

This research was supported by the Spanish MINECO (CTQ2011-28417-C02-01, CTQ2014-56362-C2-1-P, and SAF2015-65113-C2-1-R), co-financed by the European Regional Development Fund (ERDF), IMBRAIN project (FP7-REGPOT-2012-CT2012-31637-IMBRAIN), funded under the seventh Framework Programme (CAPACITIES). M.L.V. thanks CONICET for a postdoctoral grant. M.D.P. thanks the EU Social Fund (FSE) and ACIISI for a predoctoral fellowship. The authors thank graduate student José Gregorio Clemente Barrera and Ph.D. student Samuel DelgadoHernández for their helpful assistance in the synthesis of some starting materials.

\section{REFERENCES}

(1) Martín, T.; Padrón, J. I.; Martín, V. S. Strategies for the Synthesis of Cyclic Ethers of Marine Natural Products. Synlett 2013, 25, 12-32. and references therein

(2) Su, B.-N.; Takaishi, Y.; Kusumi, T. Morinols A-L, Twelve Novel Sesquineolignans and Neolignans With a New Carbon Skeleton from Morina chinensis. Tetrahedron 1999, 55, 14571-14586.

(3) Yamauchi, S.; Kawahara, S.; Wukirsari, T.; Nishiwaki, H.; Nishi, K.; Sugahara, T.; Akiyama, K.; Kishida, T. Structure-Cytotoxic Activity Relationship of Sesquilignan. Bioorg. Med. Chem. Lett. 2013, 23, 4923-4930.

(4) Akiyama, K.; Yamauchi, S.; Maruyama, M.; Sugahara, T.; Kishida, T.; Koba, Y. Antimicrobial Activity of Stereoisomers of Morinols A and B, Tetrahydropyran Sesquineolignans. Biosci., Biotechnol., Biochem. 2009, 73, 129-133.

(5) Masuda, K.; Nishiwaki, H.; Akiyama, K.; Yamauchi, S.; Maruyama, M.; Sugahara, T.; Kishida, T. Antifungal Activity of Morinol B Derivatives of Tetrahydropyran Sesquilignan. Biosci., Biotechnol., Biochem. 2010, 74, 2071-2076.

(6) (a) Erickson, K. L.; Gustafson, K. R.; Pannell, L. K.; Beutler, J. A.; Boyd, M. R. New Dimeric Macrolide Glycosides from the Marine Sponge Myriastra clavosa. J. Nat. Prod. 2002, 65, 1303-1306. (b) Rao, M. R.; Faulkner, D. J. Clavosolides A and B, Dimeric Macrolides from the Philippines Sponge Myriastra clavosa. J. Nat. Prod. 2002, 65, 386388.

(7) For the isolation of polycavernosides $\mathrm{A} 2, \mathrm{~A} 3$, and $\mathrm{B} 2$, see: (a) Yotsu-Yamashita, M.; Seki, T.; Paul, V. J.; Naoki, H.; Yasumoto, T. Four New Analogs of Polycavernoside A. Tetrahedron Lett. 1995, 36, 5563-5566. For the isolation of polycavernosides A and B, see: (b) Yotsu-Yamashita, M.; Haddock, R. L.; Yasumoto, T. Polycavernoside A: a Novel Glycosidic Macrolide from the Red Alga Polycavernosa tsudai (Gracilaria edulis). J. Am. Chem. Soc. 1993, 115, 1147-1148.

(8) Martin, H. J.; Magauer, T.; Mulzer, J. Pursuit of a Competitive Target: Total Synthesis of the Antibiotic Kendomycin. Angew. Chem., Int. Ed. 2010, 49, 5614-5626. and references therein

(9) Searle, P. A.; Molinski, T. F. Phorboxazoles A and B: Potent Cytostatic Macrolides from Marine Sponge Phorbas species. J. Am. Chem. Soc. 1995, 117, 8126-8131.

(10) Ghosh, A. K.; Anderson, D. D. Tetrahydrofuran, Tetrahydropyran, Triazoles and Related Heterocyclic Derivatives as HIV Protease Inhibitors. Future Med. Chem. 2011, 3, 1181-1197.

(11) (a) Capim, S. L.; Gonçalves, G. M.; dos Santos, G. C. M.; Marinho, B. G.; Vasconcellos, M. L. A. A. High Analgesic and AntiInflammatory in vivo Activities of Six New Hybrids NSAIAs Tetrahydropyran Derivatives. Bioorg. Med. Chem. 2013, 21, 60036010. (b) Capim, S. L.; Carneiro, P. H. P.; Castro, P. C.; Barros, M. R. M.; Marinho, B. G.; Vasconcellos, M. L. A. A. Design, PrinsCyclization Reaction Promoting Diastereoselective Synthesis of 10 New Tetrahydropyran Derivatives and in vivo Antinociceptive Evaluations. Eur. J. Med. Chem. 2012, 58, 1-11.

(12) Kharkar, P. S.; Reith, M. E. A.; Dutta, A. K. Three-Dimensional Quantitative Structure-Activity Relationship (3D QSAR) and Pharmacophore Elucidation of Tetrahydropyran Derivatives as 
Serotonin and Norepinephrine Transporter Inhibitors. J. Comput.Aided Mol. Des. 2008, 22, 1-17.

(13) (a) Surivet, J.-P.; Zumbrunn, C.; Rueedi, G.; Bur, D.; Bruyère, T.; Locher, H.; Ritz, D.; Seiler, P.; Kohl, C.; Ertel, E. A.; Hess, P.; Gauvin, J.-C.; Mirre, A.; Kaegi, V.; dos Santos, M.; Kraemer, S.; Gaertner, M.; Delers, J.; Enderlin-Paput, M.; Weiss, M.; Sube, R.; Hadana, H.; Keck, W.; Hubschwerlen, C. Novel TetrahydropyranBased Bacterial Topoisomerase Inhibitors with Potent Anti-Gram Positive Activity and Improved Safety Profile. J. Med. Chem. 2015, 58, 927-942. (b) Surivet, J.-P.; Zumbrunn, C.; Bruyère, T.; Bur, D.; Kohl, C.; Locher, H. H.; Seiler, P.; Ertel, E. A.; Hess, P.; EnderlinPaput, M.; Enderlin-Paput, S.; Gauvin, J.-C.; Mirre, A.; Hubschwerlen, C.; Ritz, D.; Rueedi, G. Synthesis and Characterization of Tetrahydropyran-Based Bacterial Topoisomerase Inhibitors with Antibacterial Activity against Gram-Negative Bacteria. J. Med. Chem. 2017, 60, 3776-3794.

(14) For the biological evaluation of THPs synthesized in our research group, see: (a) León, L. G.; Carballo, R. M.; VegaHernández, M. C.; Martín, V. S.; Padrón, J. I.; Padrón, J. M. Antiproliferative Activity of 2-Alkyl-4-Halopiperidines and 2-Alkyl-4Halo-1,2,5,6-Tetrahydropyridines in Solid Tumor Cell Lines. Bioorg. Med. Chem. Lett. 2007, 17, 2681-2684. (b) Carrillo, R.; León, L. G.; Martín, T.; Martín, V. S.; Padrón, J. M. Synthesis and Antiproliferative Activity of (2R,3R)-Disubstituted Tetrahydropyrans. Part 2: Effect of Side Chain Homologation. Bioorg. Med. Chem. Lett. 2007, 17, 780783. (c) Miranda, P. O.; León, L. G.; Martín, V. S.; Padrón, J. I.; Padrón, J. M. One-pot Synthesis and SAR Study of cis-2,6-Dialkyl-4Chloro-Tetrahydropyrans. Bioorg. Med. Chem. Lett. 2006, 16, 31353138.

(15) Muzart, J. $\mathrm{Pd}^{0}$ - and $\mathrm{Pd}^{\mathrm{II}}$-Catalyzed Oxaheterocyclization of Substrates Having Both an Allylic Leaving Group and a Hydroxylated Tether. J. Mol. Catal. A: Chem. 2010, 319, 1-29.

(16) Smith, A. B., III; Fox, R. J.; Razler, T. M. Evolution of the Petasis-Ferrier Union/Rearrangement Tactic: Construction of Architecturally Complex Natural Products Possessing the Ubiquitous cis-2,6-Substituted Tetrahydropyran Structural Element. Acc. Chem. Res. 2008, 41, 675-687.

(17) Larrosa, I.; Romea, P.; Urpí, F. Synthesis of Six-Membered Oxygenated Heterocycles through Carbon-Oxygen Bond-Forming Rreactions. Tetrahedron 2008, 64, 2683-2723.

(18) Boivin, T. L. B. Synthetic Routes to Tetrahydrofuran, Tetrahydropyran, and Spiroketal Units of Polyether Antibiotics and a Survey of Spiroketals of Other Natural Products. Tetrahedron 1987, 43, 3309-3362.

(19) For two recent overviews regarding the strategies employed in the building of THPs in the context of the synthesis of natural products, see: (a) Nasir, N. M.; Ermanis, K.; Clarke, P. A. Strategies for the Construction of Tetrahydropyran Rings in the Synthesis of Natural Products. Org. Biomol. Chem. 2014, 12, 3323-3335. (b) Clarke, P. A.; Santos, S. Strategies for the Formation of Tetrahydropyran Rings in the Synthesis of Natural Products. Eur. J. Org. Chem. 2006, 2006, 2045-2053.

(20) For the original Prins reaction, see: (a) Prins, H. J. Condensation of Formaldehyde with Some Unsaturated Compounds. Chem. Weekbl. 1919, 16, 64-74 1072-1073, 1510-1526. For the first examples of the application of the Prins cyclization to yield THPs, see: (b) Stapp, P. R. The Reaction of $\alpha$ Olefins with Paraformaldehyde and Hydrogen Halides. A Novel Tetrahydropyran Synthesis. J. Org. Chem. 1969, 34, 479-485. (c) Hanschke, E. Zur Kenntnis der Prinsschen Reaktion III. Mitteil.: Über die Reaktion von Allylcarbinol mit Aldehyden und Ketonen. Chem. Ber. 1955, 88, 1053-1061.

(21) For recent reviews in Prins cyclization, see: (a) McDonald, B. R.; Scheidt, K. A. Pyranone Natural Products as Inspirations for Catalytic Reaction Discovery and Development. Acc. Chem. Res. 2015, 48, 1172-1183. (b) Greco, S. J.; Fiorot, R. G.; Lacerda, V., Jr; dos Santos, R. B. Recent Advances in the Prins Cyclization. Aldrichimica Acta 2013, 46, 59-67. (c) Han, X.; Peh, G.; Floreancig, P. E. PrinsType Cyclization Reactions in Natural Product Synthesis. Eur. J. Org.
Chem. 2013, 2013, 1193-1208. (d) Olier, C.; Kaafarani, M.; Gastaldi, S.; Bertrand, M. P. Synthesis of Tetrahydropyrans and Related Heterocycles via Prins Cyclization; Extension to Aza-Prins Cyclization. Tetrahedron 2010, 66, 413-445. (e) Crane, E. A.; Scheidt, K. A. Prins-Type Macrocyclizations as an Efficient Ring-Closing Strategy in Natural Product Synthesis. Angew. Chem., Int. Ed. 2010, 49, 83168326. (f) Pastor, I. M.; Yus, M. The Prins Reaction: Advances and Applications. Curr. Org. Chem. 2007, 11, 925-957.

(22) For selected previous reports of our research group, see: (a) Scoccia, J.; Pérez, S. J.; Sinka, V.; Cruz, D. A.; López-Soria, J. M.; Fernández, I.; Martín, V. S.; Miranda, P. O.; Padrón, J. I. Direct Access to 2,3,4,6-Tetrasubstituted Tetrahydro- $2 \mathrm{H}$-pyrans via Tandem $\mathrm{S}_{\mathrm{N}} 2$ '-Prins Cyclization. Org. Lett. 2017, 19, 4834-4837. (b) Pérez, S. J.; Purino, M.; Miranda, P. O.; Martín, V. S.; Fernández, I.; Padrón, J. I. Prins Cyclization Catalyzed by a Fe $\mathrm{FII}^{\mathrm{II}}$ Trimethylsilyl Halide System: The Oxocarbenium Ion Pathway versus the $[2+2]$ Cycloaddition. Chem. - Eur. J. 2015, 21, 15211-15217. (c) Purino, M. A.; Ramírez, M. A.; Daranas, A. H.; Martín, V. S.; Padrón, J. I. Iron(III) Catalyzed Direct Synthesis of cis-2,7-Disubstituted Oxepanes. The Shortest Total Synthesis of (+)-Isolaurepan. Org. Lett. 2012, 14, 5904-5907. (d) Miranda, P. O.; Carballo, R. M.; Martín, V. S.; Padrón, J. I. A New Catalytic Prins Cyclization Leading to Oxa- and Azacycles. Org. Lett. 2009, 11, 357-360. (e) Miranda, P. O.; Ramírez, M. A.; Martín, V. S.; Padrón, J. I. The Silylalkyne-Prins Cyclization: Stereoselective Synthesis of Tetra- and Pentasubstituted Halodihydropyrans. Org. Lett. 2006, 8, 1633-1636.

(23) Tay, G. C.; Huang, C. Y.; Rychnovsky, S. D. Silyl Enol Ether Prins Cyclization: Diastereoselective Formation of Substituted Tetrahydropyran-4-ones. J. Org. Chem. 2014, 79, 8733-8749.

(24) Zheng, K.; Liu, X.; Qin, S.; Xie, M.; Lin, L.; Hu, C.; Feng, X. Completely $\mathrm{OH}$-Selective $\mathrm{FeCl}_{3}$-Catalyzed Prins Cyclization: Highly Stereoselective Synthesis of 4-OH-Tetrahydropyrans. J. Am. Chem. Soc. 2012, 134, 17564-17573.

(25) Elliott, M. C.; El Sayed, N. N. E.; Paine, J. S. Diastereospecific Tandem Prins Cyclisation/Rearrangement Reactions for the Desymmetrisation of Cyclohexa-1,4-dienes. Eur. J. Org. Chem. 2007, 2007, 792-803.

(26) Yang, X. F.; Mague, J. T.; Li, C. J. Diastereoselective Synthesis of Polysubstituted Tetrahydropyrans and Thiacyclohexanes via Indium Trichloride Mediated Cyclizations. J. Org. Chem. 2001, 66, 739-747.

(27) Bahnck, K. B.; Rychnovsky, S. D. Formal Synthesis of (-)-Kendomycin Featuring a Prins-Cyclization To Construct the Macrocycle. J. Am. Chem. Soc. 2008, 130, 13177-13181.

(28) Bahnck, K. B.; Rychnovsky, S. D. Rapid Stereocontrolled Assembly of the Fully Substituted C-Aryl Glycoside of Kendomycin with a Prins Cyclization: a Formal Synthesis. Chem. Commun. 2006, 22, 2388-2390.

(29) Rychnovsky, S. D.; Thomas, C. R. Synthesis of the C22-C26 Tetrahydropyran Segment of Phorboxazole by a Stereoselective Prins Cyclization. Org. Lett. 2000, 2, 1217-1219.

(30) Álvarez-Méndez, S. J.; García, C.; Martín, V. S. The Evans Aldol-Prins Cyclization: a General and Stereoselective Method for the Synthesis of 2,3,4,5,6-Pentasubstituted Tetrahydropyrans. Chem. Commun. 2016, 52, 3380-3383.

(31) As drawn herein, aldols 2 show their $N$-acyl oxazolidin-2-one and $\mathrm{R}^{2}$ groups in a trans orientation, which corresponds to a syn relative stereochemistry because the syn/anti nomenclature is established with the molecule in a zigzag projection.

(32) Evans, D. A.; Bartroli, J.; Shih, T. L. Enantioselective Aldol Condensations. 2. Erythro-Selective Chiral Aldol Condensations via Boron Enolates. J. Am. Chem. Soc. 1981, 103, 2127-2129.

(33) Morita, A.; Kuwahara, S. Enantioselective Total Synthesis of Litseaverticillols A and B. Org. Lett. 2006, 8, 1613-1616.

(34) Zhang, S.-J.; Hu, W.-X. Method for Regio- and Stereoselective Synthesis of $(E)-\beta, \gamma$-Unsaturated Acids from Aldehydes Under Solvent-Free Conditions. Synth. Commun. 2010, 40, 3093-3100.

(35) Rodeschini, V.; Boiteau, J.-G.; Van de Weghe, P.; Tarnus, C.; Eustache, J. MetAP-2 Inhibitors Based on the Fumagillin Structure. 
Side-Chain Modification and Ring-Substituted Analogues. J. Org. Chem. 2004, 69, 357-373.

(36) Andrade, C. K. Z.; Rocha, R. O.; Vercillo, O. E.; Silva, W. A.; Matos, R. A. F. DCC/DMAP-Mediated Coupling of Carboxylic Acids with Oxazolidinones and Thiazolidinethiones. Synlett 2003, 15, 2351-2352.

(37) It is described that compound $E-\alpha, \beta-3 b$ reacts in an aldol addition providing the same $\beta, \gamma$-unsaturated alcohol 2 expected to be obtained from desired $\beta, \gamma-3 \mathbf{b}$. Thus, this mixture is not a problem because a synthetic point of view is needed to achieve our goals. For a previously reported example, see: Nakamura, T.; Oshida, M.; Nomura, T.; Nakazaki, A.; Kobayashi, S. Synthetic Study of Diversifolin: The Construction of 11-Oxabicyclo[6.2.1] undec-3-ene Core Using Ring-Closing Metathesis. Org. Lett. 2007, 9, 5533-5536. Supporting Information, page S4.

(38) Raimundo, B. C.; Heathcock, C. H. Further Studies on the Anti-Selective Aldol Reaction of Chiral Imides. Synlett 1995, 1995, $1213-1214$

(39) Evans, D. A.; Tedrow, J. S.; Shaw, J. T.; Downey, C. W. Diastereoselective Magnesium Halide-Catalyzed anti-Aldol Reactions of Chiral N-Acyloxazolidinones. J. Am. Chem. Soc. 2002, 124, 392393.

(40) May, A. E.; Connell, N. T.; Dahlmann, H. A.; Hoye, T. R. A Useful Modification of the Evans Magnesium Halide Catalyzed antiAldol Reaction: Application to Enolizable Aldehydes. Synlett 2010, 2010, 1984-1986.

(41) See the Supporting Information for the mechanism of the 2oxonia-Cope rearrangement (Schemes S2 and S3, as part of other peripheral discussions). For a detailed mechanism of both Prins cyclization and the competitive processes, see: Kataoka, K.; Ode, Y.; Matsumoto, M.; Nokami, J. Convenient Synthesis of Highly Optically Active 2,3,4,6-Tetrasubstituted Tetrahydropyrans via Prins Cyclization Reaction (PCR) of Optically Active Homoallylic Alcohols with Aldehydes. Tetrahedron 2006, 62, 2471-2483.

(42) Barry, C. St. J.; Crosby, S. R.; Harding, J. R.; Hughes, R. A.; King, C. D.; Parker, G. D.; Willis, C. L. Stereoselective Synthesis of 4Hydroxy-2,3,6-trisubstituted Tetrahydropyrans. Org. Lett. 2003, 5, $2429-2432$.

(43) Stott, K.; Stonehouse, J.; Keeler, J.; Hwang, T. L.; Shaka, A. J. Excitation Sculpting in High-Resolution Nuclear Magnetic Resonance Spectroscopy: Application to Selective NOE Experiments. J. Am. Chem. Soc. 1995, 117, 4199-4200.

(44) Feuillet, F. J. P.; Niyadurupola, G.; Green, R.; Cheeseman, M.; Bull, S. D. Stereoselective Rearrangement of $\beta$-Hydroxy- $N$-acyloxazolidin-2-ones to Afford N-2-Hydroxyethyl-1,3-oxazinane-2,4-diones. Synlett 2005, 7, 1090-1094. and references therein

(45) Thomson, C. D.; Miller, T. A.; Barthen, M. T.; Dieckhaus, C. M.; Sofia, R. D.; Macdonald, T. L. The Synthesis, in vitro Reactivity, and Evidence for Formation in Humans of 5-Phenyl-1,3-oxazinane2,4-dione, a Metabolite of Felbamate. Drug Metab. Dispos. 2000, 28, 434-439.

(46) Kahns, A. H.; Møss, J.; Bundgaard, H. Improved Oral Bioavailability of Salicylamide in Rabbits by a 1,3-Benzoxazine-2,4dione Prodrug. Int. J. Pharm. 1992, 78, 199-202.

(47) Sub- and suprastoichiometric amounts of $\mathrm{FeF}_{3}, \mathrm{RuCl}_{3}, \mathrm{CeCl}_{3}$, CuI, $\mathrm{Pd}(\mathrm{OAc})_{3},\left[\mathrm{Rh}(\mathrm{OAc})_{2}\right]_{2} \cdot 2 \mathrm{H}_{2} \mathrm{O}, \mathrm{TiCl}_{4}, \mathrm{Cu}(\mathrm{OTf})_{2}, \mathrm{ZnCl}_{2}, \mathrm{TFA}$, and CSA were unsuccessfully tested to perform the Prins cyclization. In all cases, except when $\mathrm{TiCl}_{4}$ was employed, the starting material was recovered.

(48) See the Supporting Information for a comparison of their NMR spectra highlighting their characteristic signals.

(49) Guérinot, A.; Reymond, S.; Cossy, J. Ritter Reaction: Recent Catalytic Developments. Eur. J. Org. Chem. 2012, 2012, 19-28.

(50) Hu, Y.; Skalitzky, D. J.; Rychnovsky, S. D. Prins Cyclization of 4-Allyl-1,3-dioxanes Prepared from 1,3-Diol Synthons. A Rapid Entry into Functionalized Tetrahydropyrans. Tetrahedron Lett. 1996, 37, $8679-8682$.

(51) Other practical aspects of the reaction were evaluated. It was compatible with a non-inert atmosphere, and it showed robustness against the moisture: from aldol $\mathbf{2} \mathbf{b}$ and acetaldehyde, $\mathbf{5 b}$ was obtained as a sole product in $78 \%$ yield in the presence of 1 equiv of $\mathrm{H}_{2} \mathrm{O}$; when 10 equiv of $\mathrm{H}_{2} \mathrm{O}$ was added, $\mathbf{5 b}(60 \%)$ was obtained together with rearranged alcohol $\mathbf{6 b}(12 \%)$. Thorough monitoring revealed that the reaction time was $<1 \mathrm{~min}$, although a reaction time of $30 \mathrm{~min}$ was customarily selected. With aldol $\mathbf{2 a}$ and isovaleraldehyde as the starting point, no reaction was observed at $-78{ }^{\circ} \mathrm{C}$; when that reaction was performed at -18 or $0{ }^{\circ} \mathrm{C}$, 5 a was successfully obtained $(75 \%,>95: 5 \mathrm{dr})$. These temperatures are recommended in those cases in which a worse diastereoselectivity is achieved at rt.

(52) Kishi, Y.; Inagi, S.; Fuchigami, T. Prins Cyclization in Ionic Liquid Hydrogen Fluoride Salts: Facile and Highly Efficient Synthesis of 4-Fluorinated Tetrahydropyrans, Thiacyclohexanes, and Piperidines. Eur. J. Org. Chem. 2009, 2009, 103-109.

(53) Byproducts probably obtained due to an isomerization of anti$2 \mathbf{a}$ or some of its intermediates in the reaction medium. See the Supporting Information for a mechanistic proposal and a detailed analysis of the NMR spectra.

(54) Kocsis, L. S.; Benedetti, E.; Brummond, K. M. A Thermal Dehydrogenative Diels-Alder Reaction of Styrenes for the Concise Synthesis of Functionalized Naphthalenes. Org. Lett. 2012, 14, 44304433.

(55) Poulter, D. C.; Winstein, S. J. Solvolysis and Degenerate Cyclopropylcarbinyl-Cyclopropylcarbinyl Rearrangement of a Hexamethylcyclopropylcarbinyl System. J. Am. Chem. Soc. 1969, 91, 3650-3652.

(56) Reddy, B. V. S.; Yarlagadda, S.; Reddy, C. R.; Reddy, M. R.; Sridhar, B.; Satyanarayana, D.; Jagadeesh, B. Tandem Prins Strategy for the Synthesis of Spiropyrrolidine and Spiropiperidine Derivatives. Eur. J. Org. Chem. 2015, 2015, 3076-3085.

(57) Xie, Y.; Cheng, G.-J.; Lee, S.; Kaib, P. S. J.; Thiel, W.; List, B. Catalytic Asymmetric Vinylogous Prins Cyclization: A Highly Diastereo- and Enantioselective Entry to Tetrahydrofurans. J. Am. Chem. Soc. 2016, 138, 14538-14541.

(58) We speculate that $\mathrm{BF}_{3} \cdot \mathrm{OEt}_{2}$ forms a chelate with both the oxocarbenium ion and the $\mathrm{MeO}$ group, leading to a six-membered ring in the transition state that might alter the evolution of the cyclization to the bicyclic products.

(59) (a) Hinkle, R. J.; Chen, Y.; Nofi, C. P.; Lewis, S. E. Electronic Effects on a One-pot Aromatization Cascade Involving Alkynyl-Prins Cyclization, Friedel-Crafts Alkylation and Dehydration to Tricyclic Benzo $[f]$ isochromenes. Org. Biomol. Chem. 2017, 15, 7584-7593. (b) Barry, C. S.; Bushby, N.; Harding, J. R.; Hughes, R. A.; Parker, G. D.; Roe, R.; Willis, C. L. Probing the Mechanism of Prins Cyclisations and Application to the Synthesis of 4-Hydroxytetrahydropyrans. Chem. Commun. 2005, 29, 3727-3729.

(60) Jasti, R.; Anderson, C. D.; Rychnovsky, S. D. Utilization of an Oxonia-Cope Rearrangement as a Mechanistic Probe for Prins Cyclizations. J. Am. Chem. Soc. 2005, 127, 9939-9945.

(61) Crosby, S. R.; Harding, J. R.; King, C. D.; Parker, G. D.; Willis, C. L. Oxonia-Cope Rearrangement and Side-Chain Exchange in the Prins Cyclization. Org. Lett. 2002, 4, 577-580.

(62) During the synthesis of $\mathbf{5 w}$, we unsuccessfully tried to avoid the undesired production of $6 \mathrm{c}$ by repeating the reaction at different concentrations $(1$ and $0.01 \mathrm{M})$ and temperatures $\left(-78^{\circ} \mathrm{C}\right)$, but a similar proportion was always obtained. Gratifyingly, we finally were able to improve the low yield by changing DCM to $n$-hexanes as the solvent ( $50 \%$ of $5 \mathrm{w}$ and $30 \%$ of $\mathbf{6 c}$ ). As product $6 \mathrm{c}$ is a homoallylic alcohol susceptible to suffering a Prins cyclization, we tested its reactivity as an alternative precursor of bicycle $\mathbf{5 w}$. It was subjected to the standard conditions ( 2.5 equiv of $\mathrm{BF}_{3} \cdot \mathrm{OEt}_{2}, 0.1 \mathrm{M} \mathrm{DCM}$, rt) with $\mathrm{MeCHO}$ ( 1.5 equiv), and we observed that it reacted barely to yield 5w $(24 \mathrm{~h}, 40 \%)$, bringing to light the fact that the electronic and steric environment of the olefin in homoallylic alcohols 2 is key for the successful EAP cyclization.

(63) See the Supporting Information for the NMR spectra regarding the evolution of the reaction over time and for a mechanistic proposal. 
(64) Feuillet, F. J. P.; Robinson, D. E. J. E.; Bull, S. D. An (E)selective synthesis of trisubstituted $(E)-\alpha, \beta$-unsaturated acid derivatives. Chem. Commun. 2003, 17, 2184-2185.

(65) Papillon, J. P. N.; Taylor, R. J. K. The Preparation of Nonracemic Secondary $\alpha$-(Carbamoyloxy)alkylzinc and Copper Reagents. A Versatile Approach to Enantioenriched Alcohols. Org. Lett. 2002, 4, 119-122.

(66) Kende, A. S.; Kawamura, K.; DeVita, R. J. Enantioselective Total Synthesis of Neooxazolomycin. J. Am. Chem. Soc. 1990, 112, 4070-4072.

(67) Lange, H.; Huenerbein, R.; Wibbeling, B.; Fröhlich, R.; Grimme, S.; Hoppe, D. Comprehensive Experimental and Theoretical Studies of Configurationally Labile Epimeric Diamine Complexes of $\alpha$-Lithiated Benzyl Carbamates. Synthesis 2008, 2008, 2905-2918.

(68) Fuwa, H.; Saito, A.; Naito, S.; Konoki, K.; Yotsu-Yamashita, M.; Sasaki, M. Total Synthesis and Biological Evaluation of (+)-Neopeltolide and Its Analogues. Chem. - Eur. J. 2009, 15, 12807-12818.

(69) D'yakonov, V. A.; Tuktarova, R. A.; Islamov, I. I.; Khalilov, L. M.; Dzhemilev, U. M. Catalytic Cyclometallation in Steroid Chemistry IV: Efficient Method for the Synthesis of Tetrahydrothiophene, Tetrahydroselenophen and Cyclopentanone Derivatives of (5 $\alpha$ )-Cholestane. Steroids 2016, 108, 77-84.

(70) See the Supporting Information for chiral HPLC spectra of a representative compound.

(71) Thorough analyses of two-dimensional NMR, together with reproducible patterns found in the mass spectra, allowed us to unambiguously differentiate between structures of 5 and 21. GOESY analyses confirmed that both structures bear all their substituents in equatorial positions, a case analogous to that in which nonchiral substrates were used. See the Supporting Information for additional details.

(72) See the Supporting Information for the screening of Lewis acids for the enantiomeric version of Prins cyclization.

(73) See the Supporting Information for the complete list of products evaluated.

(74) De León, L.; Moujir, L. Activity and Mechanism of the Action of Zeylasterone against Bacillus subtilis. J. Appl. Microbiol. 2008, 104, $1266-1274$.

(75) An aldehyde freshly obtained through a Parikh-Doering oxidation, or a PCC-mediated oxidation, can even be used without further purification. A longer reaction time could lead to a larger amount of the undesired $\alpha, \beta$-isomer. These reactions were monitored by TLC analysis or by ${ }^{1} \mathrm{H}$ NMR analysis of aliquots taken of the reaction medium and then treated with a few drops of a $2 \mathrm{M}$ aqueous solution of $\mathrm{H}_{2} \mathrm{SO}_{4}$ and a few drops of AcOEt.

(76) A non-aqueous simplified workup is also valid: a small amount of silica gel 60 (35-70 mesh) was added, the solvent was removed in the rotavap, and the silica-supported crude was purified.

(77) When the NMR spectra were recorded using $\mathrm{CDCl}_{3}$ as the solvent, $\mathrm{NCH}\left(\mathrm{CH}_{2} \mathrm{Ph}\right) \mathrm{CH}_{2} \mathrm{OH}$ appeared as a weak br $\mathrm{s}$ in the ${ }^{13} \mathrm{C}$ spectrum, but that signal did not appear in DEPTs spectra or in HSQCed. Fortunately, its correlation appeared weakly in HMBC.

(78) When $\mathrm{CDCl}_{3}$ was employed as the solvent, $\mathrm{C}_{8 \mathrm{a}}$ did not appear in DEPTs and it was difficult to study the HSQCed because $\mathrm{H}_{8 \mathrm{a}}$ appeared as a br s. Fortunately, HMBC showed a clear correlation with $\mathrm{H}_{4 \mathrm{a}}$ and $\mathrm{H}_{8}$.

(79) When the ${ }^{13} \mathrm{C}$ spectrum was recorded at $320 \mathrm{~K}$ using $\mathrm{C}_{6} \mathrm{D}_{6}$ as the solvent, $\mathrm{NCH}\left(\mathrm{CH}_{2} \mathrm{Ph}\right) \mathrm{CH}_{2} \mathrm{OH}$ appeared as a weak br s, although its correlations were clear in HSQCed and HMBC.

(80) In the ${ }^{13} \mathrm{C}$ spectrum, this signal appears as a br s, like in other similar bicycles. However, in this product, the signal appears clearly in the DEPT90 spectrum, as well as in the HSQCed (weak correlation with $\mathrm{H}$ with $\delta$ 4.34-4.40) and the HMBC (correlation with $\left.\left(\mathrm{CH}_{3}\right)_{2} \mathrm{CH}\right)$.

(81) This is a typical fragmentation of the bicycle and does not appear, or its intensity is lower, in the mass spectrum of isomer $\mathbf{2 1 \mathbf { b }}$. (82) When $\mathrm{CDCl}_{3}$ was employed as the solvent, $\mathrm{NCH}(i-\mathrm{Pr}) \mathrm{CH}_{2} \mathrm{OH}$ was not detected in C, DEPTs, HSQCed, or HMBC. According to data for similar compounds, it should appear between 50 and $60 \mathrm{ppm}$.
(83) This is a typical fragmentation of the bicycle and does not appear, or its intensity is lower, in the mass spectrum of isomer 21c. (84) By contrast, when a solution of bicycle $\mathbf{5 a}$ in $\mathrm{Et}_{2} \mathrm{O}(0.12 \mathrm{M})$ was added to an ice-cooled suspension of $\mathrm{LiAlH}_{4}$ (9 equiv) in $\mathrm{Et}_{2} \mathrm{O}$ $(0.3 \mathrm{M})$ and the mixture was allowed to warm to rt, after $5 \mathrm{~h}$ carbamate $\mathbf{2 0}$ was obtained with a poor $5 \%$ yield together with traces of diol 19.

(85) This signal shows correlation with $\mathrm{H}_{4^{\prime}}$ in the COSY spectrum and with $\mathrm{C}_{3^{\prime}}$ and $\mathrm{C}_{4^{\prime}}$ in the $\mathrm{HMBC}$ spectrum. However, these correlations do not appear in the spectrum of its isomer, bicycle 5ah.

(86) In the mass spectrum of bicycle 5 ah, this peak may correspond to this same fragmentation but also to the typical fragmentation of a bicycle $\left(\mathrm{M}+1-\mathrm{CH}_{2} \mathrm{OH}\right)^{+}$, which explains the higher intensity observed there (66 against 1 ).

(87) In the mass spectrum of bicycle 5ah, this peak may correspond to this same fragmentation but also to the typical fragmentation of a bicycle $\left(\mathrm{M}+2-\mathrm{CH}(i-\mathrm{Pr}) \mathrm{CH}_{2} \mathrm{OH}\right)^{+}$, which explains the higher intensity observed there (94 against 2 ).

(88) In the mass spectrum of bicycle $\mathbf{5 a h}$, this fragmentation does not appear because oxazolidin-2-one is part of the bicycle and is not prone to being removed.

(89) In the mass spectrum of bicycle 5ai, these fragmentations do not appear because oxazolidin-2-one is part of the bicycle and is not prone to being removed.

(90) No products were identified in the nonpolar fractions.

(91) In addition to the typical signals due to the fragmentation of the oxazolidin-2-one of the product, no signal at $\mathrm{m} / \mathrm{z} 310$ was detected (it would have corresponded to the fragmentation $\mathrm{M}$ $\mathrm{CH}(\mathrm{Me}) \mathrm{CH}(\mathrm{Ph}) \mathrm{OH}$ of the bicyclic isomer). 